THE SPATIAL AND TEMPORAL ABUNDANCE PATTERNS

OF CHAETOGNATHS

IN THE WESTERN NORTH ATLANTIC OCEAN

by

Jerry Cheney

B.S., Lamar University

(1976)

\author{
SUBMITTED IN PARTIAL FULFILLNENT OF THE \\ REQUIREMENTS FOR THE DEGREE OF \\ DOCTOR OF PHILOSOPHY \\ at the
}

WOODS HOLE OCEANOGRAPHIC INSTITUTION

and the

MASSACHUSETTS INSTITUTE OF TECHNOLOGY

August 1982

(C) Jerry Cheney 1982

The author hereby grants to M.I.T. permission to reproduce and to distribute copies of this thesis document in whole or in part.

Signature of Author...

Joint Program in Biological Oceanography, Woods Hole Oceanographic Institution/ Massachusetts Institute of Technology, August 1982

Certified by...

Thesis Supervisor

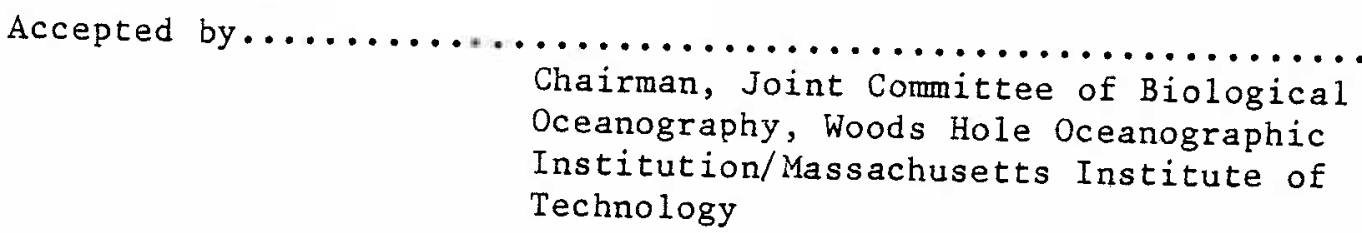




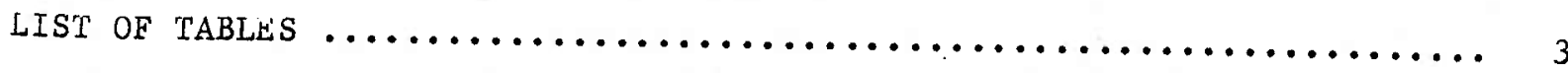

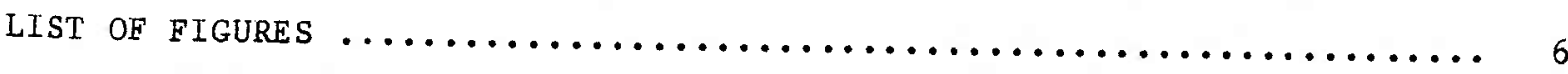

BIOGRAPHICAL NOTE $\ldots \ldots \ldots \ldots \ldots \ldots \ldots \ldots \ldots \ldots \ldots \ldots \ldots \ldots \ldots \ldots \ldots \ldots$

ALKNOWLEDGEMENTS $\ldots \ldots \ldots \ldots \ldots \ldots \ldots \ldots \ldots \ldots \ldots \ldots \ldots \ldots \ldots \ldots \ldots \ldots \ldots$

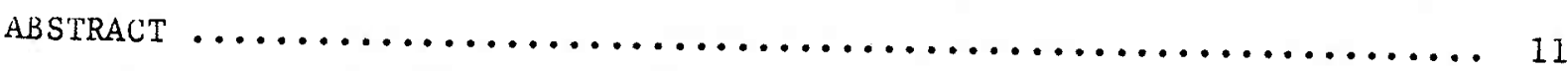

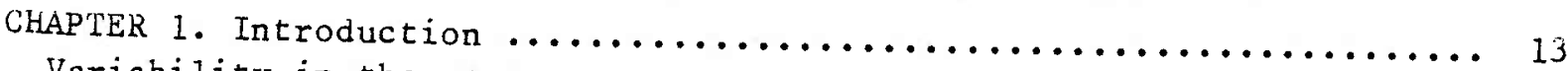

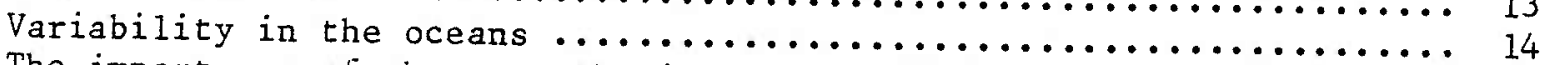

The importance of chaetognaths in pelagic commities $\ldots \ldots \ldots \ldots \ldots \ldots \ldots 18$

Predicting spatial and temporal abundance patterns $\ldots \ldots \ldots \ldots \ldots \ldots \ldots . \ldots 23$

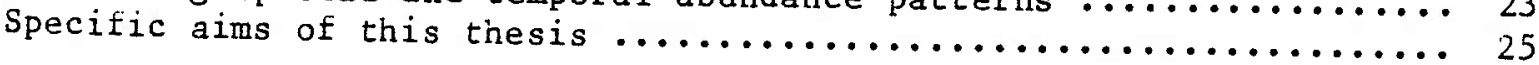

CHAPTER 2. Method $\ldots \ldots \ldots \ldots \ldots \ldots \ldots \ldots \ldots \ldots \ldots \ldots \ldots \ldots \ldots \ldots \ldots \ldots \ldots$

CHAPTER 3. Sampling Problems ........................ 43

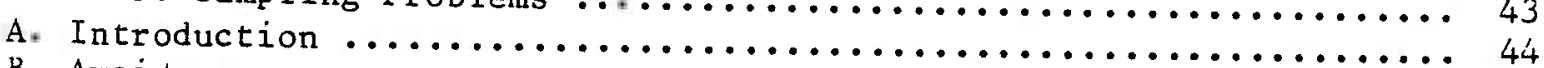

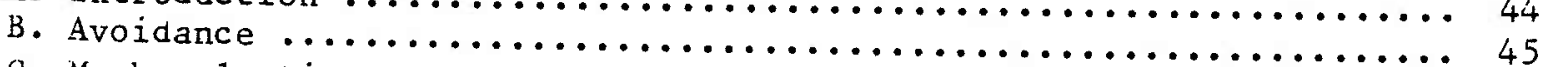

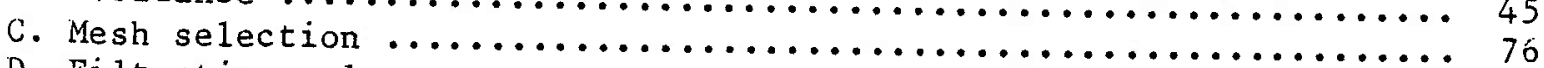

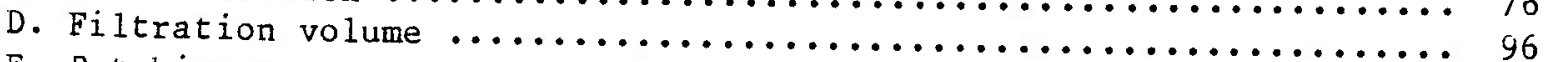

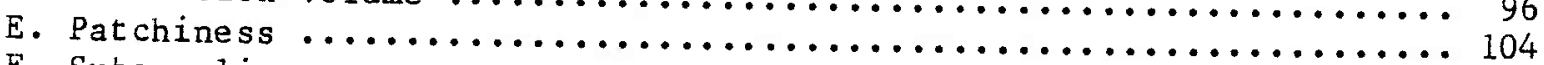

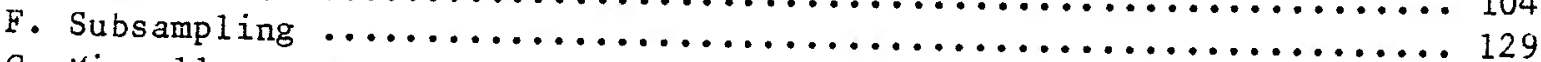

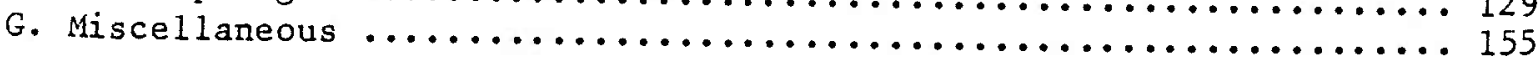

CHAPTER 4. Vertical Distribution of Chaetognaths in the

Western North Atlantic Ocean $\ldots \ldots \ldots \ldots \ldots \ldots \ldots \ldots \ldots \ldots \ldots \ldots \ldots \ldots \ldots$

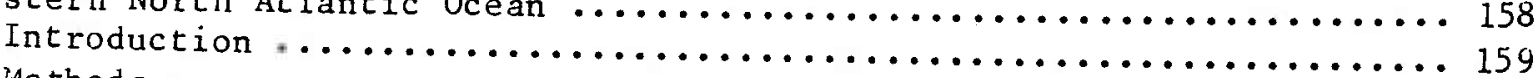

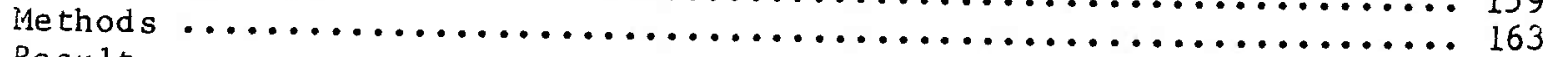

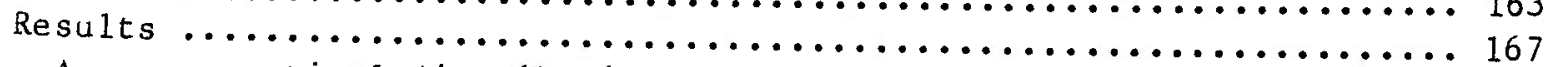

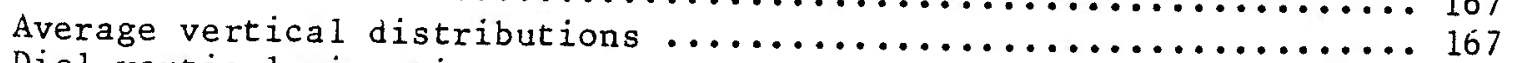

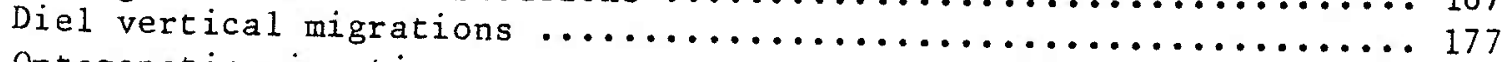

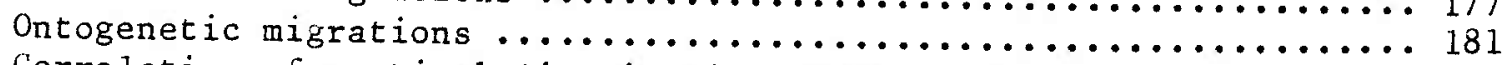

Correlation of vertical distributions with environmental

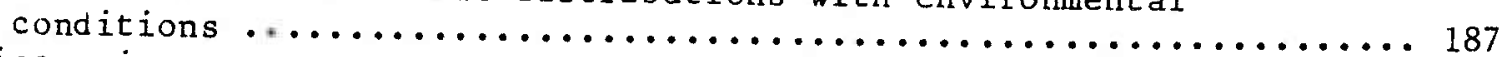

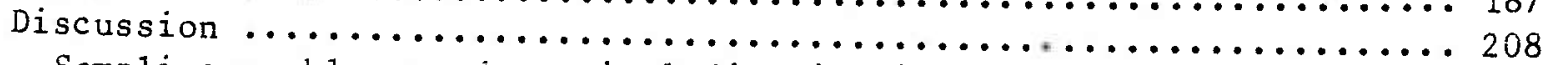

Sampling problems and vertical distributions $\ldots \ldots \ldots \ldots \ldots \ldots \ldots \ldots \ldots . \ldots \ldots$

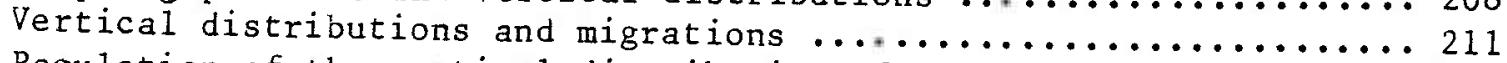

Regulation of the vertical distribution of chaetognaths $\ldots \ldots \ldots \ldots .224$

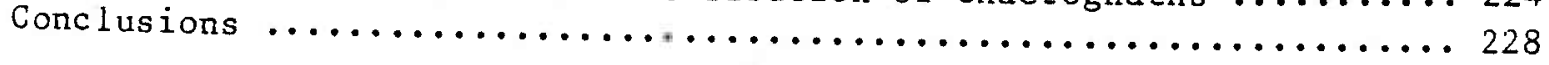

CHAPTER 5. Horizontal and Seasonal Abundance Patterns of

Chaetognaths in the Western North Atlantic Ocean ................230

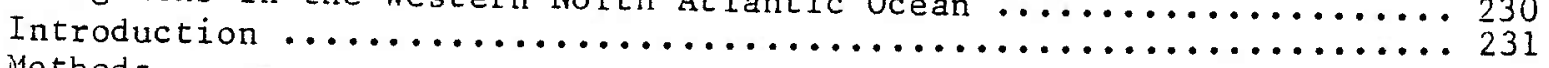

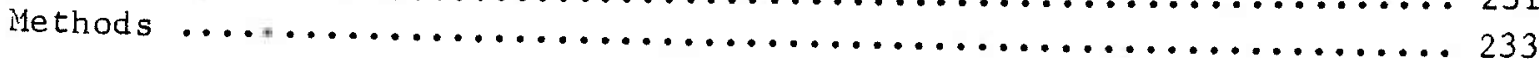




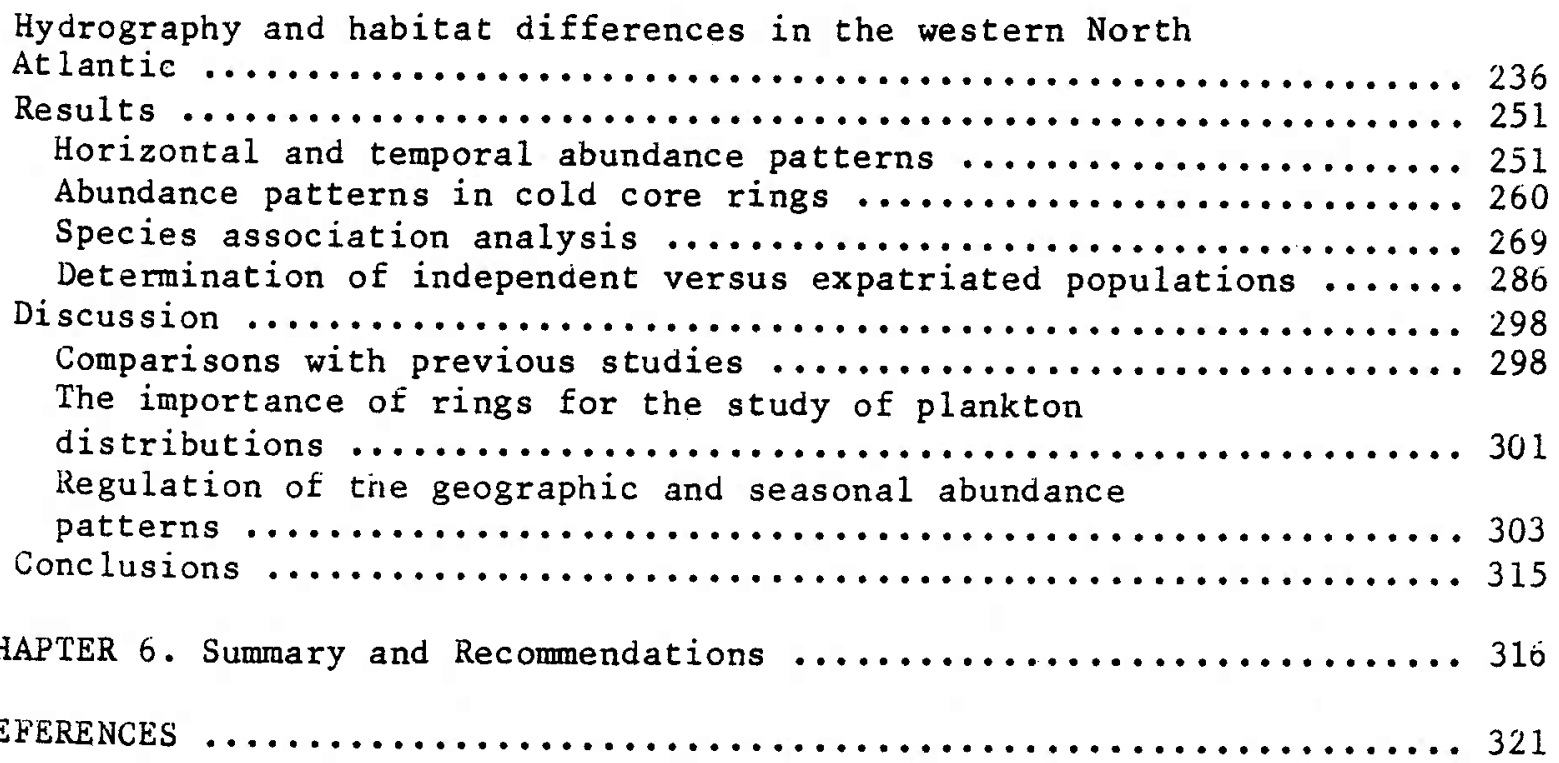


Table 1.1. Relative abundance of chaetognaths. ................ 20

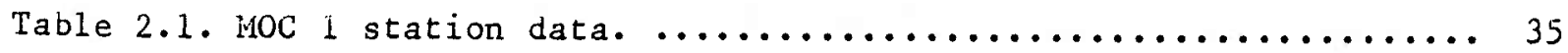

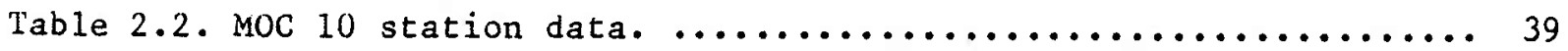

Table 3.1 . Day/night differences in chaetognath abundance. ....... 52

Table 3.2. Day/night differences in chaetognath abundance from published reports. ......................... 55

Table 3.3. Comparison of the catch of the MOC 1 and $M O C 10$ for four chaetognath species. Where two tows are shown together, the numbers $/ \mathrm{m}^{2}$ were averaged. ................... 58

Table 3.4. Ratio (R) of the catch of large nets to small nets from published reports. .......................... 62

Table 3.5. Calculated reaction distances and percent capture for individuals of three sizes from data in Table $3.4 . \ldots . . .65$

Table 3.6. Geometric mean regressions of length and width. ....... 80

Table 3.7 . Minimum retention lengths for chaetognaths caugnt by the MOC 1 and MOC 10 as predicted from the regression

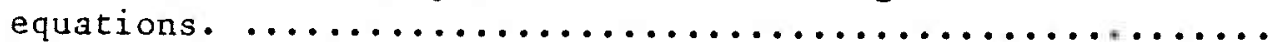

Table 3.8. Comparisons of observed and predicted retention lengths for four chaetognath species from the MOC 10. Mean length and mean plus upper $95 \%$ confidence limit from Table 3.7 are underlined for each species. .................. 88

Table 3.9. Published reports of the size of chaetognath eggs and larvae. ............................... yl

Table 3.10. Filtration characteristics of the MOC 1 nets.......... 102

Tabie 3.11. Patchiness of chaetognaths in two horizontal transects at $350 \mathrm{~m}$ in the Slope Water $(*=\mathrm{p}<0.05, * *=\mathrm{p}<0.01$,

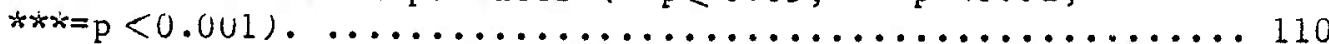

Table 3.12. Variability of chaetognath abundance estimates within day/night replicates and within hydrographic region and

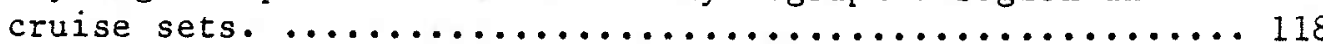


Table 3.13. Small scale variability of chaetognath abundance from

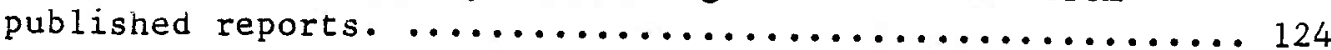

Table 3.14. Average number of splits for the eight depth intervals from the Slope Water and Northern Sargasso Sea. ......... 149

Table 3.15 . Comparison of variability due to subsampling $\left(s_{s}\right)$ and variability due to small scale patchiness $\left(s_{p}\right)$ for seventeen chaetognath species.

Table 4.1. Records of occurrence of rare chaetognath species in this study.

Table 4.2. Comparison of median deptins in the SW and NSS. Differences between regions were tested using the

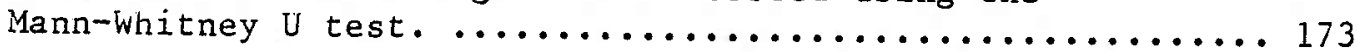

Table 4.3. Day/night differences in the vertical distribution of chaetognaths. ................................. 179

Table 4.4. Extent of the ontogenetic migrations of six chaetognaths. .. 185

Table 4.5. Correlations of median depth with environmental variables for S. lyra. The number of tows upon which the correlation coefficient was based is given in parentheses

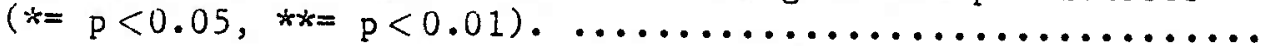

Table 4.t. Correlations of median depth with temperature (T), light $(L)$ and population abundance (P) for seven chaetognath species. The number of tows upon which the correlation coefficient was based is given in parentheses $(*=\mathrm{P}<$ $0.05, * *=p(0.01)$.

Table 4.7. Previous studies of the diel vertical migration of chaetognaths found in this study. $0=$ no diel vertical migration, $1=<50 \mathrm{~m}$ difference in day/night median depths, $2=50-100 \mathrm{~m}$ difference, $3=$ greater than $100 \mathrm{~m}$ difference, and $?=$ migration reported but no estimate of

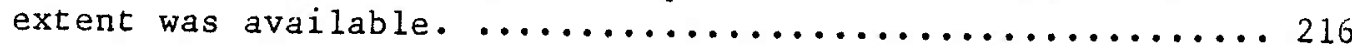

Table 4.8 . Reports of ontogenetic migrations of chaetognaths. ...... 220

Table 5.1. Mean chaetognath species abundance $\left(\right.$ no. $\left./ \mathrm{m}^{2}\right)$ in the Slope Water (SW), Northern Sargasso Sea (NSS), Gulf Stream (GS) and cold core rings (CCR). The number of tows on which the average is based is given in parentheses. R represents the ratio of SW to NSS or NSS to SW abundance, whichever is larger. $P$ is the probability of equal $S W$ and NSS abundances as tested by the Mann-Whitney $U$ test ( $* * * *=$ $p<0.001, * *=p<0.01, *=p<0.05, N S=$ not significant $). \ldots 252$ 
Table 5.2. Percent similarity of the chaetognath fauna of the Gulf Stream tows with the SW and NSS. Whittaker and Fairbanks (1958) percent similarity index ranges from 0 for no similarity to 1.0 for perfect similarity.................... 55

Table 5.3. Percent similarity of Ring $D$ and Ring Bob cores with the $\mathrm{SW}$ and NSS. Ring core abundances were averaged for each ring and/or time, and SW and NSS abundances were averaged for the same cruise on which the ring was sampled. ...... 267

Table 5.4. Results of the recurrent group analysis. Fractions in parentheses indicate the number of associations out of the total possible between the associated species and members of Group I. .......................... 270

Table 5.5. Percent variability accounted for by successive

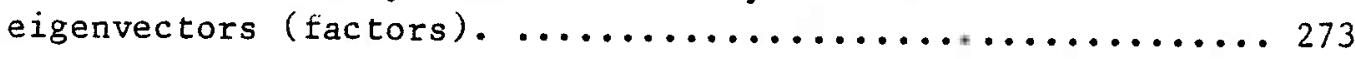

Table 5.6. Percent of each species variability accounted for after extraction of the first three factors and species communalities from factor analysis................ 276

Table 5.7. Presence of independent (t) or expatriated (-) populations in the $S W$ and $N S S . \ldots \ldots \ldots \ldots \ldots \ldots \ldots \ldots \ldots . \ldots \ldots$ 
Figure 1.1. Biogeographic provinces of the pelagic realm. ........ 16

Figure 2.1. MOC 1 station locations. ..................... 30

Figure 2.2. MOL 10 and MOC $I$ horizontal tow and cold core ring

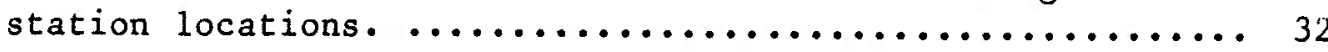

Figure 3.1. Graphical analysis of avoidance. The values along the right and botton margins of the graphs are values of the $\mathrm{x} / \mathrm{k}$ isolines. Where two tows were available from the same location, size frequency curves were averaged to reduce the

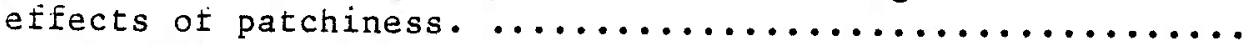

Figure 3.2. Small scale spatial distributions of chaetognaths at 350 $\mathrm{m}$ in the slope Water. Asterisks denote lost samples. ..... 113

Figure 3.3. Relationship of $s^{2} / \bar{x}$ and $\bar{x}$ for the chaetognaths from the horizontal tows in the SW. Line 2 is the geometric mean regression for all the data and Line 1 is the regression excluding the point, $\bar{x}=126, s^{2} / \bar{x}=2.4 . \ldots \ldots 115$

Figure 3.4. Relationship between the number of organisms in the total sample and the expected standard deviation of the total sample count for aliquots of $1 / 2,1 / 4,1 / 8$, and $1 / 16$.

Figure 3.5. Observed and expected standard deviations of the $1 / 2$ aliquot data as a function of the total sample count $\left(\mathrm{N}_{\mathrm{T}}\right)$.

Figure 3.6. Coefficient of variation as a function of aliquot count

(C) and total sample count $\left(\mathrm{N}_{\mathrm{T}}\right)$ for $1 / 2,1 / 4,1 / 8$, and

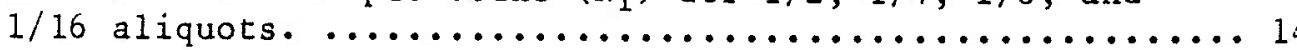

Figure 4.1. Hydrographic comparisons of the upper 1000 m of the Slope Water and Northern Sargasso Sea.

a. Temperature envelopes- $M O C I-91$ and 115 represent the Gulf Stream and MOC 1-60,61 represent the NSS in April 1977.

b. Salinity envelopes- MOC 1-60, ol represent the iNSS in April 1977.

c. Oxygen envelopes- Redrawn after Wiebe et al. (1976b).

d. Noon light envelopes- See text for methods used in calculation.

Figure 4.2. Average vertical distributions of chaetognaths in the upper $1000 \mathrm{~m}$ of the Slope water and Northern Sargasso 
Sea. Arrows indicate median depths calculated from the average vertical distributions. Depth intervals labeled with asterisks contained less than $1 \%$ of the population. . 170

Figure 4.3. Ontogenetic migrations of seven chaetognaths. Arrows indicate median depths for each length class. ........... 183

Figure 4.4. Relationships of median depths for four epipelagic species with depth of the mixed layer and temperature at

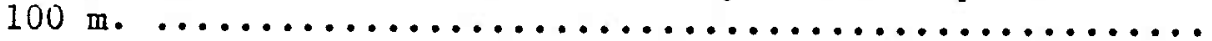

Figure 4.5. Relationships of isotherm depth with median depths for nine chaetognaths in Ring D. Asterisks denote

significant differences in median depth $(*=p<0.05$, $* * x=0<.01 . * \dot{x} \dot{x} p=<0.001)$.

Figure 4.6. Isotherm depths and median depths of ten chaetognaths plotted as a function of distance from the center of

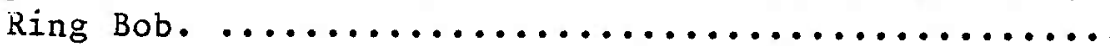

Figure 5.1. Hydrography of the western North Atlantic........... 237

Figure 5.2. Seasonal temperature variations in the upper $150 \mathrm{~m}$ of the Slope Water and Northern Sargasso Sea. The curves represent from top to bottom, $0,30,60,90,120$, and

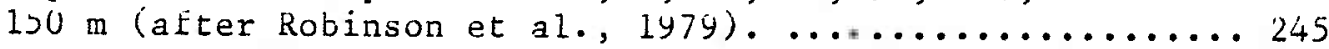

Figure 5.3. Seasonal patterns of chlorophyll a and zooplankton biomass in the SW and iNSS.

Figure 5.4. Seasonal abundance patterns of chatognaths in the SW and iss. Open circles represent 1975 cruises and closed circles represent $1976-77$ cruises. Bars indicate the

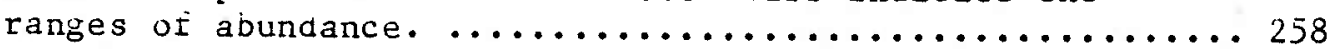

Figure 5.5. Spatial abundance patterns of chaetognaths in Ring Bob. ... 261

Figure 5.0. Temporal abundance patterns of chaetognaths in Ring D. ... 264

Figure 5.7. Three dimensional plot of species by their first three factor scores from CA $1 . \ldots \ldots \ldots \ldots \ldots \ldots \ldots \ldots \ldots \ldots \ldots$

Figure 5.8. Three dimensional plot of species by their first three

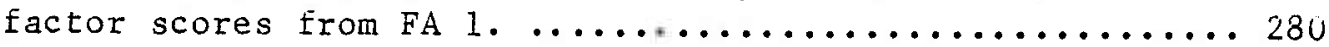

Figure 5.9. Three dimensional plot of MOCNESS stations by their first three factor scores from CA 2................ 283

Figure 5.10. Species abundance as a function of surface salinity in the $S$ iw. The solid line represents the mixing curve using NSS abundance (Table 5.1) at 36 ppt and dashed line represents mixing curve using GS adundance (Table 5.1) at 36 ppt. 
Jerry Cheney was born on June 26, 1954 in Port Arthur, Texas. As a youngster, he spent much of his time playing with his chemistry set, so early on he decided that he would be a chemist when he got older. Several near disasters while indiscriminantly mixing chemicals together convinced young Jerry that his life as a chemist might be rather short. Having seen all the Jacques Cousteau specials on television, Jerry had also become an avid SCUBA diver. He began to wonder if he could make a living by SCUBA diving. But how? He coula become a commercial diver, but no, the work was too hard and much more dangerous than his previously discarded career as a chemist. He could becone a tropical resort diving guide, but no, the risk of skin cancer was too great. He could become a marine biologist, and biology was even somewhat related to chemistry. After finishing high school in 1972, Jerry enrolled as a biology major at Lamar University in nearby Beaumont, Texas with the intention of eventually becoming a marine biologist. A three year stint as a hospital laboratory technician while at Lamar and a rapidly developing mercenary nature lead him to briefly consider a career in the medical profession, but insanity prevailed and he came to the WHOI/MIT joint program to further his goal of becoming a marine biologist. This thesis represents the culmination of his effort to become a marine biologist, and Jerry now looks forward to another long, hard struggle to remain a marine biologist. 


\section{ACKNOWLEDGEMENTS}

As is the case for most major accomplishments in life, this thesis could not have been completed without the encouragement and support of a large number of people. First and foremost, I wish to thank my family for their love and support for the past twenty eight years, and it is to my mother and stepfather, Shirley and Joe Arabie, my father, Joe Cheney, my three beautiful sisters, Carlene, Cathy and Cheryl, their husbands, David, Larry and Steve, and my inexhaustible nieces and nephews, Erica, Heather, Laura, Sean, Danny and BJ that I dedicate this thesis.

I have had the great fortune to work with some very interesting people during my stay at Woods Hole. My advisor, Peter Wiebe, was always available when needed and is responsible for most of what $I$ know about the doing of oceanography. Peter also kindly supplied his zooplankton samples and ancillary data which form the basis of this thesis. Steve Boyd's effects on my life cannot be characterized in a few short words, but I want to thank him for opening up a naive young graduate student's mind to a broader perspective of life. His sense of humor also made coming to work every day a real pleasure. Valerie Barber and Sally Strahle have been voices of reason in a sea of insanity, and their friendship is greatly appreciated.

I also owe ny thesis comittee members a debt of gratitude for the patience they showed while helping a naive and ignorant young graduate student learn about the oceans and for their diligence in reading this wordy document. Their comments and criticisms of earlier dratts of this thesis have been most helpful. Glenn Fiierl taught me much of what I know 
about physical oceanography and Will Rand taugint me the use of multivariate statistical techniques. Harding Michel taught me much about the taxonomy of chaetognaths and their distributions in the oceans. Woollcott Smith helped with the statistics and always provided words of encouragement. I have had many interesting discussions with Rich Harbison about the oceans and the organisms that inhabit them. I must also recognize the guidance $I$ received as an undergraduate at Lamar University from Philip Robertson.

To fully acknowledge the contributions of all my friends to the successful completion of this thesis would require a document longer than the thesis itself. Without the friendship they have offered, tine production of this thesis would hardly have seemed worthwhile. A special thanks goes to Rod Fujita and Dale Leavitt for helping to type the references.

I would also like to thank the Graphics Department at WHOI for their fine work under tremendous pressure.

Radio stations WGBH of Boston and WBRU of Providence also deserve my gratitude for their evening and late night jazz programing which helped me maintain my sanity during many long nights of data analysis and writing.

Financial support was provided by the Education Office at WHOI and from INSF Grants OCE-7709132 and OCE-8017248 to Peter Wiebe. The zooplankton samples were collected under previous NSF and ONR grants also to Peter Wiebe. 


\title{
THE SPATIAL AND TEMPORAL ABUNDANCE PATTERNS \\ OF CHAETOGNATHS \\ IN THE WESTERN NORTH ATLANTIC OCEAN
}

\section{by}

Jerry Cheney

\begin{abstract}
Submitted to the Woods Hole Oceanographic Institution/ Massachusetts Institute of Technology Joint Program in Biological Oceanography in partial fulfillment of the requirements for the Degree of Doctor of Philosophy August 1982
\end{abstract}

\section{ABSTRACT}

Zooplankton samples were collected with the MOCNESS (Multiple Opening/ Closing let and Environmental Sensing System) on six cruises in the western North Atlantic Ocean during the period from August 1975 to November 1977 as part of the study of the biology, physics, and chemistry of Gulf Stream cold core rings. The physical, chemical and biological properties of the Slope vater to the North of the Gulf Stream differ substantially from the $F$ operties of the Northern Sargasso Sea to the south. The purpose of this thesis was to identify the horizontal, vertical and seasonal abundance $F$. teterns of chaetognaths in this region and to relate these patterns tc environmental conditions. Twenty one chaetognath species were identified, Jf which eighteen were abundant enough for analysis of the spatial nd temporal abundance patterns.

The most important surces of sampling error in this study were mesh selection and avoidance, which bias the abundance estimates, and patchiness and subsampling, which add variability to the abundance estimates. The $0.333 \mathrm{~mm}$ mesh of the MOCNESS does not appear to sample individuals of any of the species less than $6 \mathrm{~mm}$ in length very well. Avoidance appears to be a problem only for the larger individuals $(>20$ $\mathrm{mm}$ ) of the larger species. Variability due to small scale patchiness was less for this study than for many previous studies, and this may have been due either to the location of the tows in presumably less variable open ocean regions or to the increased volume of water filtered relative to previous studies. Subsampling with the Folsom plankton sample splitter introduced more variability than predicted by the binomial distribution, and for some species the subsampling variability was greater than that due to patchiness. In spite of these sources of variability, significant spatial and temporal abundance patterns were detectable for most of the species in this region.

Vertical distributions of chaetognaths in this region were similar to those reported for these species from other regions. Nine species were classitied as epipelagic, Four were classified as mesopelagic, and five species were classified as bathypelagic. Diel vertical migrations were not detected for any of the species, but due to the depth intervals sampled, 
migrations of less than $100 \mathrm{~m}$ were not detectable. Ontogenetic migrations were detected for the seven meso- and bathypelagic species for which these migrations were examined.

The epi- and bathypelagic species were shallower in the slope water than in the Northern Sargasso Sea, suggesting that they were orienting their vertical distributions to temperature. The mesopelagic species showed little difference in vertical distribution between the two hydrographic regions. For Sagitta lyra, the only species for which size frequency data was collected from all the tows, the vertical distribution as measured by median population depth was found to be significantly correlated with average individual size. This suggests that observed differences in vertical distribution for the meso- and bathypelagic species at different times and places in the oceans may be due to changes in the size structure of the population coupled with ontogenetic migrations rather than to responses to differing environmental conditions. It may be possible to correlate the vertical distributions of size or sexual maturity classes with environmental variables such as temperature and light.

The differences in hydrography between the slope Water and the Northern Sargasso Sea were also reflected in the species abundances. Seven species were significantly more abundant in the Slope water, nine were significantly more abundant in the Northern Sargasso Sea, and two species showed no significant differences between the two regions. Recurrent group analysis did not delineate these groupings, and it is suggested that quantitative methods employing abundance estimates be used to detect associations among species. Ordination analysis (e.g. factor analysis and correspondence analysis) was found to delineate the species groupings adequately.

The hydrographic differences in abundance for these chaetognaths were most likely related to differences in food abundance and temperature structure of the water column between the slope Water and Northern Sargasso Sea.

Seasonal abundance differences were not detected for the seven slope Water species, yet seasonal abundance differences are expected in such temperate regions. Most of the Northern Sargasso Sea species were most abundant in the spring, and this was presumably related to the 1 ate winter-early spring peak in primary productivity and zooplankton biomass in this region.

The spatial and temporal abundance patterns of chaetognaths in Gulf Stream cold core rings were also studied. The patterns were similar to predictions made on the basis of their abundance differences between the Slope Water and Northern Sargasso Sea, but a few species showed anamolous patterns. Further studies of the chaetognath fauna of both warm and cold core rings should provide invaluable insight into the processes regulating the spatial and temporal abundance patterns of these organisms.

Thesis Supervisor: Dr. Peter H. Wiebe, Associate Scientist, Biology Department, Woods Hole Oceanographic Institution. 
CHAPTER 1

Introduction 
Variability in the oceans

Variability in the abundance of organisms occurs on a wide variety of space and time scales. Haury et al. (1978) identified a number of spatial scales on which aggregated or patchy abundance patterns have been observed in the oceans, and they defined these spatial scales as mega-scale $(>3000$ $\mathrm{km})$, macro-scale $(1000-3000 \mathrm{~km})$, meso-scale $(100-1000 \mathrm{~km})$, coarse scale $(1-100 \mathrm{~km})$, fine scale $(1-1000 \mathrm{~m})$ and micro-scale $(1-100 \mathrm{~cm})$. These scales may be thought of as the diameters of patches of plankton organisms. Mega-scale pattern refers to the broad geographic distributions of plankton organisms, while macroscale pattern is manifested as abundance differences within geographic provinces. Familiar examples of meso-scale pattern are large edaies and broad upwelling events. Coarse scale pattern is less well known, but includes plume-type upwelling and island wakes. Pattern on the fine and micro-scales is what is generally referred to as small scale patchiness, the study of which has received much attention in the past fifty years.

Temporal variability in abundance has also been documented on a wide range of time scales. Seasonal abundance patterns are perhaps the best known but variations on both longer and shorter time scales are also known. Changes in the abundance and composition of the English Channel plankton over the past fifty years (Southward, 1980) and fluctuations in the abundance of commercial fishes off Japan and California over periods of fifty to one hundred years (Uda, 1961; Soutar and Isaacs, 1974) are examples of abundance variations on longer time scales.

The goal of ecologists is not only to document the spatial and temporal 
abundance patterns of organisms, but also to understand the interactions of the organisms with the environment which produce the observed patterns (Krebs, 1978; Frost, 1974). We must understand the processes regulating the distribution and abundance of organisms if we are to protect the living resources of the sea from destruction aue to man's increasing utilization of the oceans.

The broad geographic distributions (mega-scale pattern) are now well known for many pelagic organisms (McGowan, 1971; Backus et al., 1977), but relatively little is known of the mechanisms by which these patterns are regulated. We know that there are relatively few patterns of distribution (Figure 1.1), suggesting that similar processes are operating to limit the distributions of a wide variety of organisms, from diatoms to tuna. We also know that these patterns are associated with distinct hydrographic regions (McGowan, 1974; Backus et al., 1977; Reid et a1., 1978), but isolating the important processes operating to produce the patterns has proven difficult.

This thesis is an attempt to gain insight into the processes regulating the geographic abundance patterns of plankton. The region of the western North Atlantic Ocean south of New England provides a unique setting for the study of geographic distributions, with the Gulf Stream separating the Slope Water, representing a Transitional province, from the Northern Sargasso Sea, representing a Central province. Chaetognaths were chosen as the subjects of this study because their geographic distributions correspond well with those of other plankters and because they are important members of pelagic ecosystems. Although no definitive statements can be made, several hypotheses are offered regarding the regulation of 
Figure 1.1. Biogeographic provinces of the pelagic realm. 


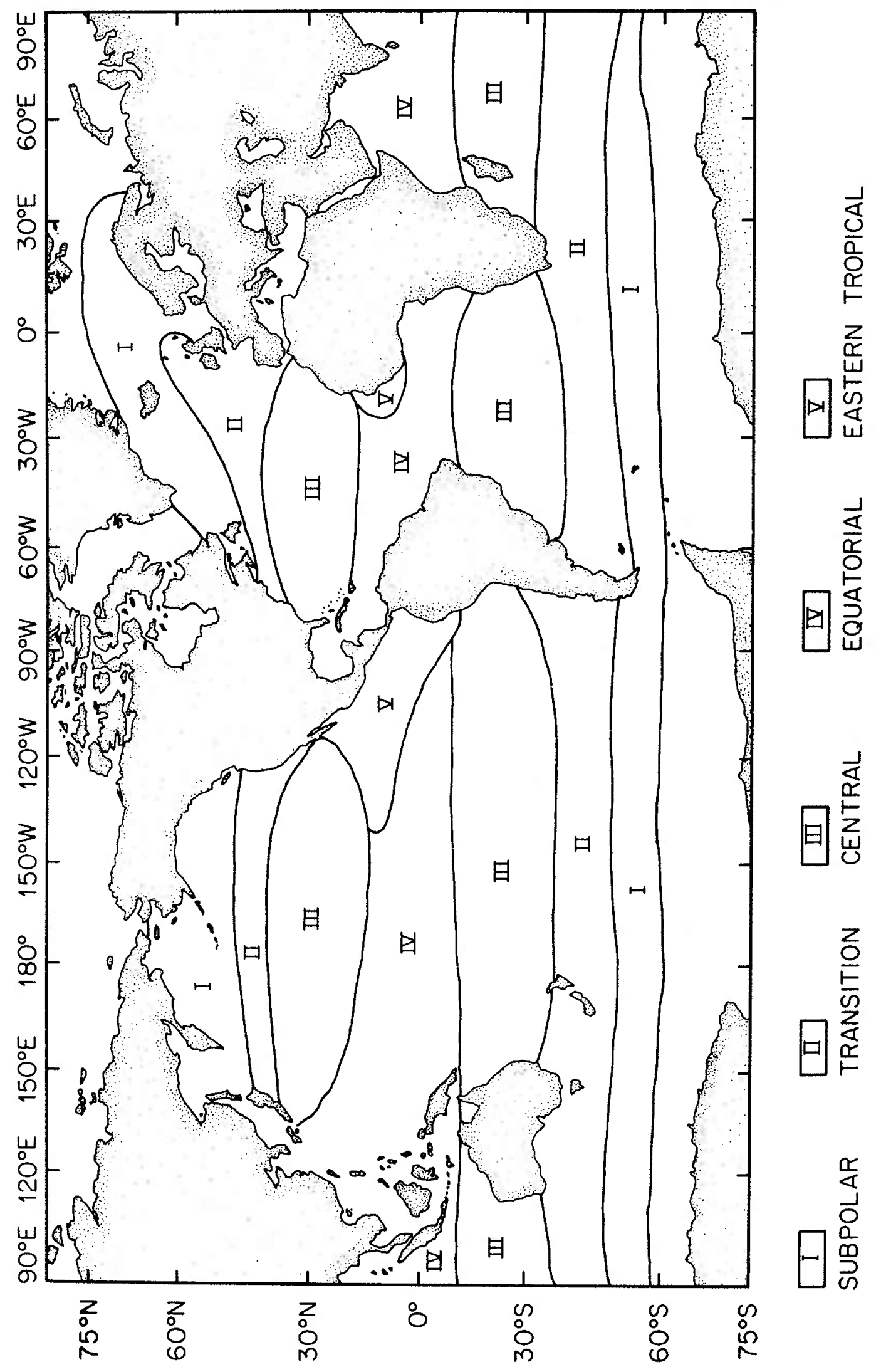


chaetognath distributions in this region.

The importance of chaetognaths in pelagic ecosytems

Chaetognaths are small, arrow-shaped creatures and are ubiquitous components of the marine plankton, being found from estuaries to the open ocean and from the sea surface to the ocean depths. There are approximately sixty species distributed among seven pelagic genera, and there are several species in one benthic genus, Spadella. There has been much speculation about the affinities of the phylum Chaetognatha, but there is no conclusive evidence linking them with any particular invertebrate group. Alvarino (1965) and Ghirardelli (1968) provide excellent reviews of the biology of chaetognaths.

Given that oceanographic studies are becoming increasingly costly, what justification can be made for studying the distribution and abundance of chaetognaths? They are one of the most abundant zooplankton groups, and they are important as predators, competitors and prey in pelagic ecosystems. Because chaetognaths are relatively well sampled by plankton nets (see Chapter 3) and their geographic distributions are similar to those of other zooplankters (Bieri, 1959; MCGowan, 1971), they may serve as general models for understanding the processes regulating the patterns of many of these zooplankters.

Chaetognaths quite often rank second in abundance to copepods among the macroplankton, and they are almost invariably among the top five numerically dominant groups (Table 1.1). Ot the twelve studies from Table 
1.1 in which the abundances of various zooplankton groups were measured, chaetognaths comprised on average $4.6 \%$ of the total numbers and $11.7 \%$ of the total biomass. The percentages given in Table 1.1 may not be entirely accurate because not all members of the plankton are sampled well by any particular net. Reeve (1968) reviewed several other previous reports and found that chaetognaths were second in abundance to copepods in eight of twelve cases when seasonal swarms of temporary plankton were excluded. He estimated that chaetognath biomass averaged about $30 \%$ of the copepod biomass from these studies, and from the studies in Table 1.1, chaetognaths averaged $34 \%$ of the copepod biomass.

Large numbers do not necessarily imply that chaetognaths are important in the functioning of pelagic ecosystems. Little information is available to assess their functional importance, but as discussed below, what information there is suggests that they are important as predators, competitors and prey.

Chaetognaths are carnivores, with copepods being their most frequent prey (Pearre, 1976; Sullivan, 1980). They appear to feed upon any organism of the appropriate size, including fish larvae, and chaetognath predation may be an important source of mortality for commercial fish larvae (Lebour, 1922, 1923; Kuhlmann, 1977).

Four studies provide quantitative estimates of the importance of chaetognaths as predators. McLaren (1969) estimated that Sagitta elegans production was 49,62 and $196 \mathrm{mgC} / \mathrm{m}^{2} /$ year in the inner, middle and outer basins of Ogac Lake, a landlocked fiord on Baffin Island, during 1957 and was $318 \mathrm{mgC} / \mathrm{m}^{2} /$ year in the middle basin during 1962 . These production values corresponded to $8,9,26$ and $48 \%$ of the estimated herbivore 
Table 1.1. Relative abundance of chaetognaths. 


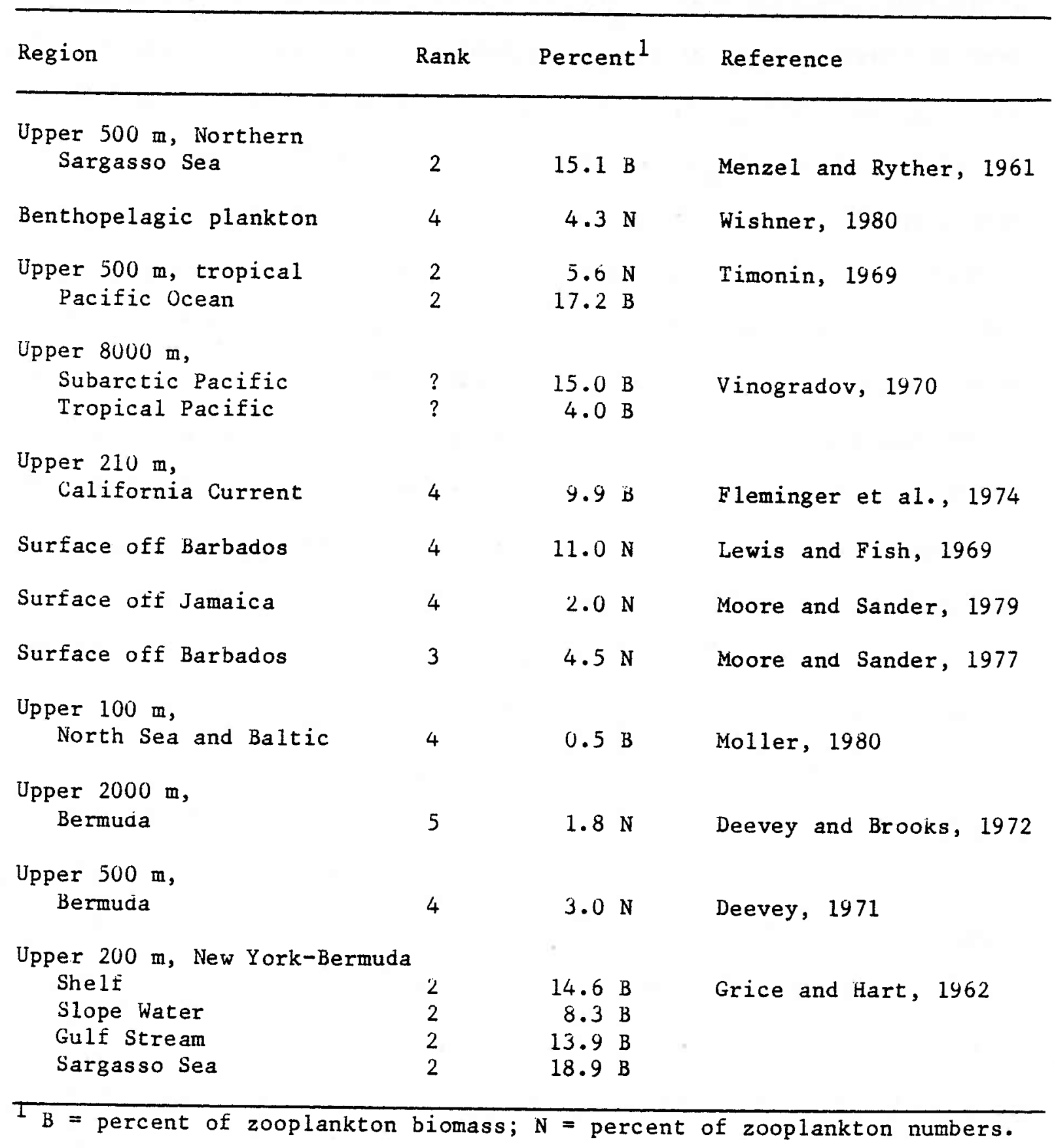


production. Assuming that losses due to unassimilated food and metabolism were at least as large as the $\underline{\mathrm{S}}$. elegans production values (Sameoto, 1971, 1973), S. elegans was responsible for the consumption of at least 16, 18, 52 and $90 \%$ of the herbivore production at various places and times in Ogac Lake.

Sameoto (1971) measured the metabolic requirements and growth (production) of S. elegans in St. Margaret's Bay, Nova Scotia, and by approximating assimilation efficiency and copepod production, he arrived at an estimate of 0.7 to $1.1 \%$ for its consumption of copepod production. Using similar measurements from Bedford Basin, Nova Scotia, Sameoto (1973) estimated that the metabolic requirements and growth of $\underline{S}$ elegans accounted for $36 \%$ of the copepod production. Assuming an assimilation efficiency of $80 \%$ (Cosper and Reeve, 1975) yields a $45 \%$ consumption of copepod production by $\underline{S}$. elegans in Bedford Basin.

Reeve and Baker (1975) estimated S. hispida production in Card Sound and Biscayne Bay, Florida to be 730 and $1752 \mathrm{mgC} / \mathrm{m}^{2} /$ year, respectively. Approximating the metabolic requirements and assimilation efficiency of $\underline{S}$. hispida and the production of zooplankton, they concluded that this species consumed 100 and $50 \%$ of the zooplankton production in the sound and bay, respectively.

Although many approximations were required in the above calculations, we may conclude that chaetognaths can be responsible for consuming a large fraction of the secondary production in pelagic ecosystems.

Many other pelagic organisms feed upon the same sizes and types of prey as chaetognaths. Competitors for these same food resources may include medusae, siphonophores, ctenophores, predaceous crustaceans, polychaetes, 
heteropods, midwater fish, commercial planktivorous fish and fish larvae. Published reports, of which there are greater than one hundred, of chaetognath predators include medusae, siphonophores, predaceous copepods, euphausiids, amphipods and decapods, midwater fish and many species of commercial fish.

All of the above information suggests that chaetognaths are important members of pelagic food webs, where they transfer energy and materials from copepods to fish.

Predicting spatial and temporal abundance patterns

Our understanding $0 \%$ the processes regulating an organism's spatial and temporal abundance pat:erns may be tested by our ability to predict these patterns from a knowlerge of the processes. This has lead to the development of mathematical models for predicting abundance as a function of time and/or space. These models serve other useful purposes as wellthey may be used as an aid in managing exploited populations, they allow us to test the importance of various processes in determining the abundance patterns, and at the very least they force us to state our hypotheses in a concise and unambiguous form.

Models of the general form, $\mathrm{dN} / \mathrm{dt}=\mathrm{f}$ (organism's interactions with its environment), have long been used for predicting plankton abundance (N) as a function of time ( $t$ ) (Cushing, 1959; Riley, 1963; Steele, 1974). In principle, such a model may be expanded to predict the temporal abundance patterns of an organism at any location in the ocean if we know the 
functional relations on the right side of the equation and the spatial variations of the important environmental variables. As discussed earlier, the goal of ecologists is to specify the functional relationships on the right side of the equation.

Both biological and physical processes may affect spatial and temporal abundance patterns in the oceans, and the biological processes may be further subdivided into population dynamics and behavioral processes. Behavioral processes are primarily important in determining small scale spatial patterns including vertical distributions, while population dynamic processes are responsible for temporal and larger spatial abundance patterns. A variety of physical processes may alter the spatial and temporal abundance patterns of plankton, and on the larger space scales advection and mixing are the most important processes.

Although these patterns may be studied separately, the vertical distributions and geographic patterns are not actually independent. As will be discussed below, the choice of vertical distributions may affect the population dynamics and the vertical distributions of chaetognaths are a function of their population size structures (see Chapter 4), which are determined by their population dynamics.

Population dynamics refers to the effects of the opposing forces of reproduction and mortality on population abundance, and therefore environmental interactions which affect the reproductive and/or mortality rates of the population must be understood.

Because environmental conditions change rapidly with depth in the oceans, it is important that we understand how zooplankters select their vertical distributions. This knowledge must be combined with a knowledge 
of the effects of these environmental conditions on reproduction and mortality in order to predict the geographic and temporal abundance patterns.

How do we determine what environmental factors are involved and how they act to produce the observed abundance patterns? Ecologists have typically used three methods- field sampling, laboratory experimentation and field experimentation. Field studies are primarily important for identifying the spatial and temporal abundance patterns, but they are also useful in relating abundance patterns to environmental conditions. Observed correlations may be used to develop hypotheses regarding the processes regulating the abundance patterns. Laboratory experiments provide controlled conditions for assessing the effects of various environmental factors on reproductive and mortality rates of populations. Field experiments provide a less controlled situation, but may avoid many of the criticisms of maintaining populations in the laboratory. It may be argued that all three methods are necessary to adequately assess the processes regulating the spatial and temporal abundance patterns of organisms.

Specific aims of this thesis

It was originally hoped that laboratory experiments could be carried out to determine the effects of various environmental factors on the reproductive and mortality rates of one or more chaetognaths. This information was to be used to develop a mathematical model to predict the 
geographic and seasonal abundance patterns for comparison with the patterns from field samples. Because of the difficulties of culturing open ocean chaetognatns this proved impossible. The resulting thesis is an attempt to use the sampling method for the study of the geographic abundance patterns of chaetognaths. It is hoped that hypotheses developed from these observation may be tested with further field studies and laboratory experiments, should culturing of open ocean chaetognaths become possible.

What specific questions can be asked from the samples used in this study? The questions were separated into three categories, and these form the basis of the results presented in Chapters 3,4 , and 5 .

\section{Chapter 3}

How reliable are the abundance estimates from the field samples? How might we improve our sampling methods to reduce sampling error?

\section{Chapter 4}

What are the vertical distributions of chaetognaths in this region and how do they compare with their vertical distributions in other regions? Do chaetognaths vertically migrate on a daily or ontogenetic basis? Can the vertical distributions be correlated with environmental parameters, and if so, can these relations be used to predict the vertical distributions under a variety of environmental conditions?

\section{Chapter 5}

Are the geographic abundance patterns related to hydrography, and if 
so, what environmental factors are these patterns correlated with?

Are there seasonal abundance patterns, and if so, what environmental factors are they correlated with?

Can Gulf Stream rings provide us with any information concerning the regulation of the spatial and temporal abundance patterns? 
CHAPTER 2

Methods 
Zooplankton samples were collected on six cruises during the period from August 1975 to November 1977 in the western North Atlantic Ocean as part of a multidisciplinary study of the biology, chemistry and physics of Gulf Stream cold core rings (The Ring Group, 1981). Station locations are shown in Figures 2.1 and 2.2 . The four hydrographic regions sampled were Slope Water (SW), Northern Sargasso Sea (NSS), Gulf Stream (GS) and Gulf Stream cold core rings (CCR). Although the geographic boundaries of these hydrographic regions fluctuate, SW and NSS stations were easily identifiable from vertical profiles of temperature and salinity (Figure 4.1). GS and CCR stations were intermediate in temperature and salinity characteristics.

Zooplankton samples were collected with the MOCNESS (Multiple Opening/Closing Net and Environmental Sensing System). The MOCNESS is a multiple opening and closing net with a rectangular mouth opening $1.4 \mathrm{~m}$ high by $1.0 \mathrm{~m}$ wide. When towed at a $45^{\circ}$ angle to the vertical, the effective mouth area is $1 \mathrm{~m}^{2}$. The MOCNESS has nine nets, each constructed of $0.333 \mathrm{~mm}$ Nitex gauze. It was typically fished by deploying the system to $1000 \mathrm{~m}$ with the first net open, and the subsequent eight nets sampled, in oblique tows, sequential depth intervals to the surface. Depth intervals sampled were usually $1000-850 \mathrm{~m}, 850-700 \mathrm{~m}, 700-550 \mathrm{~m}$, 550-400 m, 400-300 m, 300-200 m, 200-100 m, and 100-0 m. On the two cruises in 1975, the water column was sampled from 800 m to the surface in eight $100 \mathrm{~m}$ intervals. Occasionally, depth intervals were altered to sample around specific hydrographic structures such as the seasonal thermocline. Tows were most often taken as day/night pairs in order to examine the possible vertical migrations of zooplankton. Station data are 
Figure 2.1. MOC 1 station locations. 


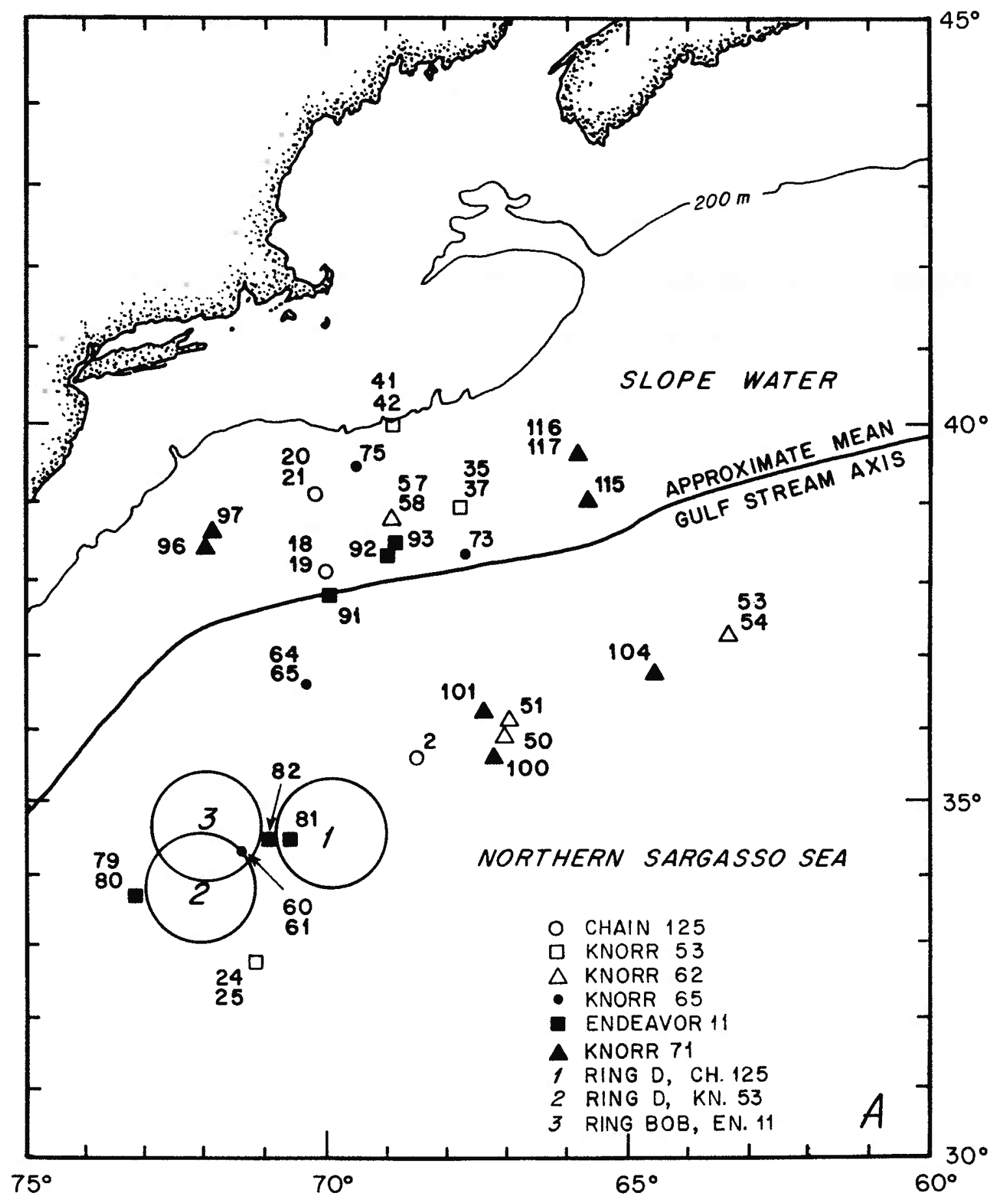


Figure 2.2. MOC 10 and MOC 1 horizontal tow and cold core ring station locations. 


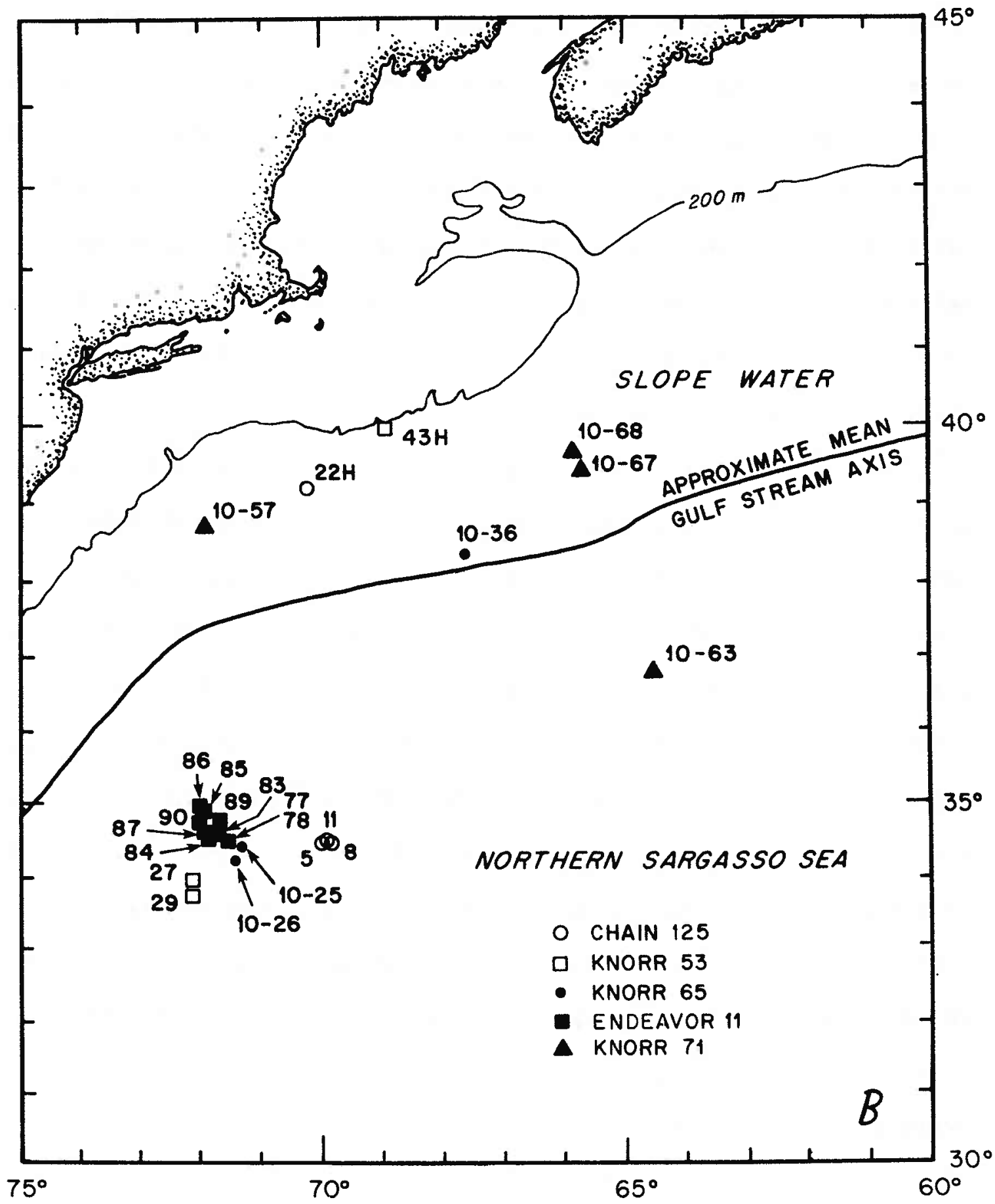


provided in Table 2.1 .

The MOCNESS was equipped with electronic sensors on the net frame which transmitted data to the ship via acoustic cable. Temperature, salinity, depth, $\mathrm{O}_{2}$ concentration, net angle and flowmeter counts were displayed in real time aboard the ship. The nets were opened and closed by a signal sent via the acoustic cable. Flowmeter readings from a TSK flowmeter on the net frame were used to calculate the speed of the net through the water and the volume of water filtered by each net. Net speed was maintained at 20.5 knots $(100 \pm 25 \mathrm{~cm} / \mathrm{s})$ and each net usually filtered between 500 and $1000 \mathrm{~m}^{3}$ of water. Further details of the MOCNESS were presented by Wiebe et a1. (1976a).

In addition to the MOCNESS samples, zooplankton/micronekton samples were collected with a larger version of the MOCNESS, the MOCNESS 10 . These samples were used in analyses of the avoidance capabilities of chaetognaths and mesh selection by the two nets. The terms MOCNESS and MOC 1 will be used to refer to the small net system, while the term MOC IO refers to the large net system. The MUC 10 has a rectangular mouth opening $4.5 \mathrm{~m}$ high by $3.2 \mathrm{~m}$ wide, and when towed at a $45^{\circ}$ angle to the vertical, the effective mouth area is $10 \mathrm{~m}^{2}$. Both the MOC 1 and MOC 10 are towed from the top of the net frame, thus eliminating towing cables and bridles directly in front of the net. The MOC 10 was fitted with five nets of 3.0 mm nylon netting, and was typically fished by deploying the system to 1000 m with the first net open, with the subsequent four nets sampling $250 \mathrm{~m}$ depth intervals to the surface. MOC 10 nets usually filtered between 15,000 and $30,000 \mathrm{~m}^{3}$ of water. MOC 10 station locations are shown in Figure 2.2, and station data is presented in Table 2.2. Samples from both 
Table 2.1. MOC 1 station data. 


\begin{tabular}{|c|c|c|c|c|c|c|c|c|c|}
\hline Cruise & MOC1非 & Region & & Date & & $\begin{array}{l}\text { Time } \\
(E S T)\end{array}$ & $\begin{array}{l}\text { Depth rang } \\
\text { sampled }\end{array}$ & (m) & Missing samples \\
\hline \multirow[t]{8}{*}{$\operatorname{Ch} 125$} & 2 & NSS & 4 & Aug & 75 & $2300 N$ & $0-800$ & $a$ & \\
\hline & 5 & $\mathrm{CCK}$ & 7 & Aug & 75 & $2100 \mathrm{~N}$ & $0-800$ & a & \\
\hline & 8 & CCR & 8 & Aug & 75 & $2300 \mathrm{~N}$ & $100-800$ & $b$ & \\
\hline & 11 & CCR & 10 & Aug & 75 & $1000 D$ & $0-800$ & a & Net 1 \\
\hline & $\begin{array}{l}18 \\
19\end{array}$ & $\begin{array}{l}\text { SW } \\
\text { SW }\end{array}$ & $\begin{array}{l}15 \\
15\end{array}$ & $\begin{array}{l}\text { Aug } \\
\text { Aug }\end{array}$ & $\begin{array}{l}75 \\
75\end{array}$ & $\begin{array}{l}1300 \mathrm{D} \\
2100 \mathrm{~N}\end{array}$ & $\begin{array}{l}0-800 \\
0-800\end{array}$ & $\begin{array}{l}a \\
a\end{array}$ & \\
\hline & 20 & SW & 16 & Aug & 75 & $2100 \mathrm{~N}$ & $0-800$ & c & \\
\hline & 21 & SW & 17 & Aug & 75 & $1000 \bar{D}$ & $0-800$ & $d$ & \\
\hline & 22 & SW & 17 & Aug & 75 & $1300 D$ & 350 & e & Nets 1 and 2 \\
\hline \multirow[t]{8}{*}{$\operatorname{Kn} 53$} & $\begin{array}{l}24 \\
25\end{array}$ & $\begin{array}{l}\text { NSS } \\
\text { NSS }\end{array}$ & $\begin{array}{l}17 \\
17\end{array}$ & $\begin{array}{l}\text { Nov } \\
\text { Nov }\end{array}$ & $\begin{array}{l}75 \\
75\end{array}$ & $\begin{array}{l}1500 \mathrm{D} \\
2200 \mathrm{~N}\end{array}$ & $\begin{array}{l}0-800 \\
0-800\end{array}$ & $a$ & \\
\hline & 27 & $\mathrm{CCK}$ & 21 & Nov & 75 & 1000D & $0-800$ & $a$ & \\
\hline & 29 & $\mathrm{CCR}$ & 21 & Nov & 75 & $2000 \mathrm{~N}$ & $0-800$ & a & \\
\hline & 35 & SW & 27 & Nov & 75 & $2000 \mathrm{~N}$ & $0-800$ & a & Net 5 \\
\hline & 37 & SW & 28 & Nov & 75 & $1100 \mathrm{D}$ & $0-800$ & a & Net 5 \\
\hline & 41 & SW & 30 & Nov & 75 & $1900 \mathrm{~N}$ & $0-800$ & $a$ & Net 8 \\
\hline & 42 & SW & 1 & Dec & 75 & $0900 \mathrm{D}$ & $0-800$ & a & \\
\hline & 43 & SW & 1 & Dec & 75 & $1200 D$ & 350 & e & \\
\hline \multirow[t]{6}{*}{ Kno 2} & 50 & NSS & 7 & Dec & 76 & $1500 \mathrm{D}$ & $0-1000$ & \pm & \\
\hline & 51 & NSS & 8 & Dec & 76 & $2300 \mathrm{~N}$ & $0-1000$ & $\mathrm{f}$ & Net 3 \\
\hline & 53 & NSS & 14 & Dec & 76 & $2200 \mathrm{~N}$ & $0-1000$ & $g$ & \\
\hline & 54 & NSS & 15 & Dec & 76 & $1500 \mathrm{D}$ & $0-700$ & $\mathrm{~h}$ & \\
\hline & $\begin{array}{l}57 \\
58\end{array}$ & SW & 19 & Dec & 76 & $0100 \mathrm{~N}$ & $0-1000$ & $\mathrm{f}$ & \\
\hline & & & 20 & DeC & 76 & $1300 \mathrm{D}$ & $0-1000$ & $\mathrm{f}$ & Net 3 \\
\hline
\end{tabular}


Table 2.1. (cont'd).

\begin{tabular}{|c|c|c|c|c|c|c|c|c|c|}
\hline \multirow{2}{*}{$\begin{array}{l}\text { Cruise } \\
\text { Kn65 }\end{array}$} & \multirow{2}{*}{$\begin{array}{l}\text { MOC 1 \# } \\
60 \\
61\end{array}$} & \multicolumn{2}{|c|}{ Region } & \multicolumn{2}{|c|}{ Date } & \multirow{2}{*}{$\begin{array}{l}\begin{array}{l}\text { Time } \\
\text { (EST) }\end{array} \\
\begin{array}{l}1100 \mathrm{D} \\
2200 \mathrm{~N}\end{array}\end{array}$} & \multicolumn{2}{|c|}{$\begin{array}{l}\text { Depth range } \\
\text { sampled (m) }\end{array}$} & \multirow{2}{*}{ Missing samples } \\
\hline & & $\begin{array}{l}\text { NSS } \\
\text { NSS }\end{array}$ & $\begin{array}{l}11 \\
13\end{array}$ & $\begin{array}{l}\text { Apr } \\
\text { Apr }\end{array}$ & $\begin{array}{l}77 \\
77\end{array}$ & & $\begin{array}{l}0-1000 \\
0-1000\end{array}$ & $\begin{array}{l}f \\
f\end{array}$ & \\
\hline & $\begin{array}{l}64 \\
65\end{array}$ & $\begin{array}{l}\text { NSS } \\
\text { NSS }\end{array}$ & $\begin{array}{l}18 \\
18\end{array}$ & $\begin{array}{l}\text { Apr } \\
\text { Apr }\end{array}$ & $\begin{array}{l}77 \\
77\end{array}$ & $\begin{array}{l}1400 \mathrm{~N} \\
2000 \mathrm{~N}\end{array}$ & $\begin{array}{l}0-1000 \\
0-1000\end{array}$ & $\begin{array}{l}\mathfrak{f} \\
\dot{E}\end{array}$ & \\
\hline & 73 & SW & 28 & Apr & 77 & $1100 \mathrm{D}$ & $0-1000$ & $\mathbf{f}$ & \\
\hline & 75 & SW & 28 & Apr & 77 & $2200 \mathrm{~N}$ & $0-1000$ & $\mathrm{f}$ & \\
\hline \multirow[t]{14}{*}{ En 11} & 77 & CCR & 31 & Ju1 & 77 & $2400 \mathrm{~N}$ & $0-1000$ & $\mathrm{f}$ & \\
\hline & 78 & $\mathrm{CCR}$ & 1 & Aug & 77 & $1800 \mathrm{D}$ & $0-1000$ & $\mathrm{E}$ & \\
\hline & 79 & NSS & 2 & Aug & 77 & $1200 D$ & $0-1000$ & 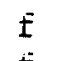 & \\
\hline & 80 & NSS & 3 & Aug & 77 & $0100 \mathrm{~N}$ & $0-1000$ & $\ddot{ \pm}$ & \\
\hline & $\begin{array}{l}81 \\
82\end{array}$ & $\begin{array}{l}\text { NSS } \\
\text { NSS }\end{array}$ & $\begin{array}{l}5 \\
6\end{array}$ & Aug & $\begin{array}{l}77 \\
77\end{array}$ & $\begin{array}{l}2200 \mathrm{~N} \\
1000 \mathrm{D}\end{array}$ & $\begin{array}{l}0-1000 \\
0-1000\end{array}$ & $f$ & \\
\hline & 83 & CCR & 6 & Aug & 77 & $2200 N$ & $0-1000$ & $\mathfrak{E}$ & \\
\hline & 84 & CCR & 7 & Aug & 77 & O900D & $0-1000$ & $\mathrm{f}$ & \\
\hline & 85 & $\mathrm{CCR}$ & 7 & Aug & 77 & $2200 \mathrm{~N}$ & $0-1000$ & $E$ & \\
\hline & 86 & CCR & 8 & Aug & 77 & $0300 \mathrm{~N}$ & $0-1000$ & $\mathrm{f}$ & \\
\hline & 87 & CCR & y & Aug & 77 & $1000 D$ & $0-1000$ & $\mathrm{f}$ & \\
\hline & 89 & CCR & 12 & Aug & 77 & $0100 \mathrm{~N}$ & $0-1000$ & $f$ & \\
\hline & 90 & CCR & 14 & Aug & 77 & $1000 \mathrm{D}$ & $0-1000$ & $f$ & \\
\hline & 91 & GS & 15 & Aug & 77 & $1000 D$ & $0-1000$ & $\mathrm{E}$ & \\
\hline & $\begin{array}{l}92 \\
93\end{array}$ & $\begin{array}{l}\text { SW } \\
\text { SW }\end{array}$ & $\begin{array}{l}15 \\
16\end{array}$ & $\begin{array}{l}\text { Aug } \\
\text { Aug }\end{array}$ & $\begin{array}{l}77 \\
77\end{array}$ & $\begin{array}{l}2200 \mathrm{~N} \\
1000 \mathrm{D}\end{array}$ & $\begin{array}{l}0-1000 \\
0-1000\end{array}$ & $\begin{array}{l}f \\
f\end{array}$ & \\
\hline
\end{tabular}


Table 2.1. (cont'd).

\begin{tabular}{|c|c|c|c|c|c|c|c|}
\hline Cruise & MOC 1非 & Region & & Date & $\begin{array}{l}\text { Time } \\
\text { (EST) }\end{array}$ & $\begin{array}{l}\text { Depth range } \\
\text { sampled (m) }\end{array}$ & Missing samples \\
\hline \multirow[t]{5}{*}{$\operatorname{kn} 71$} & $\begin{array}{l}96 \\
97\end{array}$ & $\begin{array}{l}\text { SW } \\
\text { SW }\end{array}$ & $\begin{array}{l}23 \\
23\end{array}$ & $\begin{array}{l}\text { Oct } 77 \\
\text { Oct } 77\end{array}$ & $\begin{array}{l}1100 \mathrm{D} \\
2300 \mathrm{~N}\end{array}$ & $\begin{array}{ll}0-1000 & i \\
0-1000 & i\end{array}$ & Net 4 \\
\hline & $\begin{array}{l}100 \\
101\end{array}$ & $\begin{array}{l}\text { INSS } \\
\text { NSS }\end{array}$ & $\begin{array}{l}30 \\
31\end{array}$ & $\begin{array}{l}\text { Oct } 77 \\
\text { Oct } 77\end{array}$ & $\begin{array}{l}2100 \mathrm{~N} \\
1500 \mathrm{D}\end{array}$ & $\begin{array}{ll}0-1000 & f \\
0-1000 & f\end{array}$ & \\
\hline & 104 & NSS & 4 & Nov 77 & $1800 \mathrm{~N}$ & $0-1000 \mathrm{f}$ & \\
\hline & 115 & GS & 14 & Nov 77 & $1300 \mathrm{D}$ & $0-1000 \mathrm{f}$ & \\
\hline & $\begin{array}{l}116 \\
117\end{array}$ & $\begin{array}{l}\text { SW } \\
\text { SW }\end{array}$ & $\begin{array}{l}15 \\
16\end{array}$ & $\begin{array}{l}\text { Nov } 77 \\
\text { Nov } 77\end{array}$ & $\begin{array}{l}2100 \mathrm{~N} \\
1300 \mathrm{D}\end{array}$ & $\begin{array}{ll}0-1000 j \\
0-1000 j\end{array}$ & \\
\hline
\end{tabular}

a. $800-700,700-600,600-500,500-400,400-300,300-200,200-100,100-0$.

b. $800-755,755-700,700-600,600-500,500-400,400-300,300-200,200-100$.

c. $800-650,650-500,500-400,400-300,300-200,200-100,100-50,50-0$.

d. $800-650,650-500,500-400,400-300,300-200,200-100,100-65,65-0$.

e. Horizontal tow at $350 \mathrm{~m}$.

f. $1000-850,850-700,700-550,550-400,400-300,300-200,200-100,100-0$.

g. $1000-850,850-700,700-550,550-350,350-300,300-200,200-100,100-0$.

h. $700-600,600-500,500-400,400-300,300-200,200-100,100-50,50-0$.

i. $1000-850,850-700,700-550,550-400,400-250,250-150,150-50,50-0$.

j. $1000-800,800-600,600-400,400-300,300-200,200-100,100-50,50-0$. 
Table 2.2. MOC 10 station data. 


\begin{tabular}{|c|c|c|c|c|c|c|c|c|}
\hline Cruise & MOC 10 作 & Region & & Date & $\begin{array}{l}\text { Time } \\
\text { (EST) }\end{array}$ & $\begin{array}{l}\text { Depth range } \\
\text { sampled (m) }\end{array}$ & MOC 1 & replicates \\
\hline \multirow[t]{3}{*}{ Kn65 } & 25 & NSS & 13 & Apr 77 & $0100 N$ & $0-1000$ & & 61 \\
\hline & 26 & NSS & 13 & Apr 77 & $1500 \mathrm{D}$ & $0-1000$ & & 60 \\
\hline & 36 & SW & 28 & Apr 77 & $1400 D$ & $0-1000$ & & 73 \\
\hline \multirow[t]{4}{*}{$\mathrm{Kn} 71$} & 57 & SW & 24 & Oct 77 & $0200 \mathrm{~N}$ & $0-1000$ & & 96,97 \\
\hline & 63 & NSS & 4 & Nov 77 & $2300 \mathrm{~N}$ & $0-1000$ & & 104 \\
\hline & 67 & SW & 15 & Nov 77 & $1400 D$ & $0-1000$ & & 117 \\
\hline & 68 & SW & 16 & Nov 77 & $0200 \mathrm{~N}$ & $0-1000$ & & 116 \\
\hline
\end{tabular}


the MOC 1 and MOC 10 were stored in glass jars and preserved in a 5-10\% buffered formalin in seawater solution.

Chaetognaths were removed from each sample, or in some cases from as small as $1 / 16$ aliquot, and identified to species. Aliquots were taken, using a Folsom plankton sample splitter, most frequently from samples from the upper $200 \mathrm{~m}$ because chaetognaths were most abundant there. Unidentified specimens were classified as either Eukrohnia spp. or Unidentified.

Alvarino's (1969) study of the Atlantic chaetognaths was the major taxonomic reference used, although an unpublished key developed by Dr. Harding B. Michel of the University of Miami was also of great help. References used to identify species untreated in or unclear from the above sources were Grant (1967) for Sagitta serratodentata and S. tasmanica and Dallot and Ducret (1969) for S. megalophthalma.

Total sample counts for each species were converted to number $/ 1000 \mathrm{~m}^{3}$ for each depth interval sampled (number/1000 $\mathrm{m}^{3}=$ number/sample $\mathrm{x}$ $1000 /$ volume of water filtered). The numbers/1000 $\mathrm{m}^{3}$ per depth interval were used in the analysis of the vertical distributions.

The numbers $/ 1000 \mathrm{~m}^{3}$ for each depth interval were integrated for each tow to provide an estimate of the total population abundance for each species under a square meter of ocean surface. The numbers $/ \mathrm{m}^{2}$ were for the upper 800 or $1000 \mathrm{~m}$ of the water column, depending upon the maximum depth of sampling. These values were used in the analysis of the horizontal and temporal abundance patterns.

Because these abundance data were not normally distributed, either normalizing transformations or nonparametric statistics were used to test 
for differences in abundance. Details of the specific methods used for data analysis are presented with the results of those analyses. 
CHAPTER 3

Sampling Problems 


\section{A. Introduction}

We need to understand the quantitative significance of errors associated with towed net sampling in order to determine how representative our samples are of the abundance of zooplankton in nature. Several sources of error have been recognized as affecting estimates of zooplankton abundance, and the most important of these are avoidance, mesh selection, clogging of the net, patchiness and subsampling. The first three sources of error affect the accuracy and the last two affect the precision of the abundance estimates. Those errors which affect the accuracy of the abundance estimates generally result in an underestimate of the true abundance, while the errors which affect precision make it difficult to detect significant spatial and temporal abundance differences.

Because the work presented here involves the analysis of spatial and temporal differences in chaetognath abundance and because very little information is available regarding sampling errors for chaetognaths, I* have examined the importance of several of these errors for chaetognaths as sampled by the MOC 1. An understanding of the quantitative significance of these errors will aid in the interpretation of the MOC 1 data and should be useful in designing future sampling studies. 
B. Avoidance

Introduction

Nets and associated cables may produce visual, audible and tactile cues as they are towed through the water. It is clear that many zooplankters can sense the approach of a net and can swim out of the path of the net before capture (Clutter and Anraku, 1968). We need to understand the quantitative significance of avoidance if we are to relate abundances of organisms in net samples to actual abundances in the environment. Avoidance of nets by pelagic organisms has been detected primarily by three methods. Some plankters, especially larval fish and euphausiids, are caught in greater abundance at night than during the day (Ah1strom, 1954; Brinton, 1967; Wiebe et al., 1982). This observation is most easily attributed to avoidance as long as the entire vertical range of the species has been sampled. Fleminger and clutter (1965) caught fewer mysids under lighted conditions than in the dark, but copepods were caught equally well under all light conditions tested. It is likely that plankters with image forming eyes (e.g. larval fish, euphausiids, mysids) can visually detect and avoid nets, while those plankters with simple ocellus type eyes (e.g. copepods and chaetognaths) must depend upon other cues such as near-field water displacements or far-field pressure variations (sound).

Further evidence of avoidance is the fact that larger nets catch greater numbers of individuals per unit volume of water filtered than do smaller nets (Fleminger and Clutter, 1965; Murphy and Clutter, 1972). As 
theoretical studies of avoidance have shown, this observation can be attributed to avoidance if one assumes that an organism's swimming speed (escape velocity) and its distance from the net when it perceives the net (reaction distance) remain the same, regardless of net size (Barkley, 1972; Lava1, 1974).

More recently, size distributions of pelagic organisms have been compared with theoretical predictions in an effort to quantify avoidance behavior (Barkley, 1972; Wiebe et al., 1982).

A11 three of the above methods were applied in an analysis of the avoidance capabilities of chaetognaths. I have analyzed my own data, collected over the past several years in the western North Atlantic Ocean, and I have analyzed data from several published sources. There were no significant day-night differences in abundance for chaetognaths and thus the hypothesis that chaetognaths do not visually detect and avoid towed nets is accepted. Comparisons of the catches of different sized nets and comparisons of size frequency data with theoretical predictions yield equivocal results, but they suggest that avoidance is a major problem only for the larger individuals of the larger species. Well designed field experiments combined with laboratory behavior experiments should be undertaken to further assess the avoidance capabilities of chaetognaths.

Methods

Zooplankton samples were collected as discussed in Chapter 2. Seventeen MOC 1 tow pairs were available for analysis of the day and night 
abundances of eighteen chaetognath species. Fewer than seventeen pairs were available for any species because samples representing particular depth intervals were lost on occasion (Table 2.1) and if the lost sample occurred within the depth range normally occupied by a particular species, that day/night pair was eliminated from the analysis for that species. Day/night pairs were tested for greater night abundance by the nonparametric Wilcoxon signed-rank test. In addition, the night abundances were sumned and divided by the sum of the day abundances for each species to provide a measure of the extent to which each species was more abundant at night.

Day/night abundances of chaetognaths were also examined from data presented by Motoda and Anraku (1954, 1955), Gehringer (1962), and Tranter et al. (1968). Where the author has not performed a test of significance for greater night abundance, I have performed a t-test on the $\log _{10}(\mathrm{X}+1)$ transformed day and night abundances.

Five MOC 1 and MOC 10 sets (Table 2.2) were available for analysis of the abundance of four chaetognath species caught by two different sized nets. Only individuals larger than $20 \mathrm{~mm}$ were included in this analysis because the abundance of individuals smaller than $20 \mathrm{~mm}$ in the MOC 10 dropped rapidly to zero and it was thus assumed that $20 \mathrm{~mm}$ was the minimum size caught by the $3 \mathrm{~mm}$ mesh of the MOC 10. I have also compared abundances from different sized nets as reported by Winsor and Clarke (1940), Tranter et al. (1968) and Sands (1978). In each case, I have calculated the ratio of abundance in the larger net to the smallest net for use in further calculations as discussed below.

Equations derived from theoretical studies of avoidance (Barkley, 1972; 
Laval, 1974) were used to quantitatively assess the importance of avoidance. Barkley relates the probability of capture (P) to the radius of the net mouth $(R)$, the speed of the net (U), the escape velocity of the organism ( $u$ ) and the reaction distance of the organism ( $x$ ) as follows:

$P=\left(1-x \cdot u / R\left(U^{2}-u^{2}\right)^{1 / 2}\right)^{2}$

(1) Barkley (6)

Laval provided a similar equation relating the probability of capture (p) to the diameter of the net (D), $U, u$ and $x$ as before, and the angle of escape $(\theta)$ :

$P=((D-(2 \cdot x \cdot u \cdot \operatorname{SIN} \theta) /(U-u \cdot \cos \theta)) / D)^{2}$

(2) Lava1 (4)

The difference between these equations is Barkley's assumption that the organisms adopt the angle of flight $\left(\theta^{*}\right)$ most likely to result in successful avoidance. As Laval points out, however, $\theta^{*}$ depends upon the speed of the net, and it is not likely that any zooplankter can account for the speed of the net in its choice of an escape angle. When the catches of two nets towed at the same speed but of different size are compared, their ratio (d) should be as follows:

$d=\left(\left(D_{1}-2 \cdot x \cdot V\right) / D_{1}\right)^{2} /\left(\left(D_{2}-2 \cdot x \cdot V\right) / D_{2}\right)^{2}$ (3) Lava 1 (8)

where $D_{1}$ and $D_{2}$ are the diameters of the two nets and $V=$ $\mathrm{uSIN} \theta /(U-u \operatorname{Cos} \theta)$. This equation $\operatorname{can}$ be rearranged to produce: 
$2 \cdot x \cdot V=\left(D_{1} \cdot D_{2}\left(1-d^{1 / 2}\right)\right) /\left(D_{2}-D_{1} \cdot d^{1 / 2}\right)$

Given two nets of diameter $D_{1}$ and $D_{2}$, the ratio of their catches (d) and the speed of the net (U), we can calculate the reaction distance ( $x$ ) by assuming values for the other two unknowns, $\theta$ and $u$. The ratios of larger net catch to smaller net catch which were greater than one from data presented by Winsor and Clarke (1940), Tranter et al. (1968) and Sands (1978) were used to calculate $2 \mathrm{xV}$. I have assumed, as Laval did, that $\theta$ is $45^{\circ}$ and, as Barkley has, that $u$ is ten times the body length of the avoiding organism for computing the value of $v$. We know very little about the escape velocity of zooplankters, but as Barkley pointed out, using the equation $u=10 \mathrm{~L}$, where $\mathrm{L}$ is the body length, provides a reasonable estimate of the maximum swimming velocity for organisms for which it has been measured. Net diameters and net speeds were provided by Winsor and Clarke, Tranter et al. and Sands. Once $x$ was calculated, its value was substituted into equation (2) with the known and as sumed values for the other variables, and then $P$, the probability of capture, was determined. Although the results of this analysis may not be entirely accurate due to inadequacies in some of the assumptions made, the results should provide us with an order of magnitude estimate of our ability to catch chaetognaths with towed nets.

As a final estimate of the avoidance behavior of chaetognaths, I have applied Barkley's graphical method of analysis (Barkley, 1972) to size frequency data for four chaetognath species. Barkley provides a plot of P, the probability of capture, versus $u / U$ on which are plotted isolines of $\mathrm{x} / \mathrm{R}$ (Figure 3.1). This graph is based upon equation (1) presented earlier. 
In order to estimate $x$ and $P$, we need to know $u, U$ and $R$. The net speed (U) for the MOC 1 was about $100 \mathrm{~cm} / \mathrm{s}$ and the net radius ( $R$ ) $c$ an be approximated as $50 \mathrm{~cm}$. The escape velocity (u) was again approximated as $u=10 \mathrm{~L}$, where $\mathrm{L}$ is the length of the chaetognath in $\mathrm{cm}$, and $\mathrm{u} / \mathrm{U}$ is thus $a$ measure of the size structure of the population.

The size frequency distributions of four chaetognath species were plotted on semilog graph paper. The ordinate of these plots is the number of individuals under a square meter of ocean surface and the abscissa is $u / U$, the transformed measure of chaetognath length as discussed earlier. The size frequency graph was placed over the graph in Figure 3.1 and was moved up and down until the size frequency curve best matched one of the $x / R$ isolines. Since we know the radius of the net $(R)$, we can calculate the reaction distance ( $x$ ) from the value of $x / R$ which best matches the size frequency curve. This analysis assumes that the true size distribution of the population has equal numbers in all size classes and that deviations from the uniform size distribution are due to avoidance as a function of size. For most aquatic invertebrates, the abundance within size classes usually decreases with increasing size, which is the same pattern predicted by avoidance theory. It is likely that this analysis overestimates the reaction distance $(x)$ and underestimates the probability of capture (P). 
Results

Day/night comparisons

These results of the day/night abundance comparisons were consistent with the hypothesis that chaetognaths do not visually detect and avoid towed nets (Table 3.1 ). The mean night to day ratio for all species (excluding S. helenae and Eukrohnia spp.) was 1.02 . S. serratodentata was the only species which was significantly more abundant at night than during the day. We expect $5 \%$ of such tests to be significant at the 0.05 level, and $5 \%$ of the nineteen tests is 0.95 . Because only one test was significant and because of the nearness of the night-day ratio (1.10) for S. serratodentata, I conclude that there were no greater night than day abundances beyond expectation.

S. helenae was much more abundant at night as indicated by its night/day ratio of 8.26 , but night abundance was not significantly greater than day abundance by the Wilcoxon signed-ranks test. S. helenae was not very abundant in this study and its large day/night ratio was the result of its relatively large abundance in MOC 1-75, a night tow. If this tow is excluded from the data, the night/day ratio becomes 1.03 .

S. decipiens was interesting in that it was significantly more abundant in the day than in the night tows. If a two tailed Wilcoxon signed-rank test is applied to these data (testing for either a greater night or day abundance), S. decipiens remains as the only significant departure from equal day/night abundance. Again, we expect $5 \%$ of the 19 tests to be significant at the 0.05 level, and the observed value of one significant 
Table 3.1. Day/night differences in chaetognath abundance. 


\begin{tabular}{|c|c|c|c|}
\hline Species & $\begin{array}{l}\text { \# of day- } \\
\text { night pairs }\end{array}$ & $\frac{\text { Night abundance }}{\text { Day abundance }}$ & $\begin{array}{c}\text { Night abundance greater } \\
\text { than day abundance }\end{array}$ \\
\hline E. bathypelagica ${ }^{1}$ & 10 & 0.97 & NS \\
\hline E. fowleri ${ }^{1}$ & 10 & 1.22 & NS \\
\hline E. hamata ${ }^{1}$ & 12 & 0.82 & NS \\
\hline K. pacifica & 12 & 1.18 & NS \\
\hline K. subtilis & 13 & 0.81 & NS \\
\hline P. draco & 13 & 1.10 & NS \\
\hline S. bipunctata & 12 & 0.86 & NS \\
\hline S. decipiens & 15 & 0.80 & $\mathrm{NS}^{2}$ \\
\hline S. enflata & 15 & 1.36 & NS \\
\hline S. helenae & 5 & 8.26 & NS \\
\hline S. hexaptera & 15 & 0.92 & NS \\
\hline S. 1 yra & 14 & 0.90 & NS \\
\hline S. macrocephala ${ }^{1}$ & 14 & 0.93 & NS \\
\hline S. $\underline{\text { maxima }}^{1}$ & 10 & 1.11 & NS \\
\hline S. minima & 13 & 1.35 & NS \\
\hline S. planctonis ${ }^{1}$ & $\dot{i}$ & 1.24 & NS \\
\hline S. serratodentata & 14 & 1.10 & $0.025<p<0.05$ \\
\hline S. tasmanica & 7 & 0.74 & NS \\
\hline Eukrohnia spp. 1,3 & 12 & 1.19 & NS \\
\hline $\begin{array}{l}1 \text { Species which oc } \\
2 \text { S. decipiens was } \\
3 \text { These were probal }\end{array}$ & $\begin{array}{l}\text { ur below dep } \\
\text { significant } 1 \\
\text { ly juvenile }\end{array}$ & $\begin{array}{l}\text { of sampling (se } \\
\text { more abundant du } \\
\text { fowleri. }\end{array}$ & $\begin{array}{l}\text { text). } \\
\text { ng the day (see text). }\end{array}$ \\
\hline
\end{tabular}


departure was within the expected range. I conclude that there were no day/night differences in abundance for any of these species.

Our sampling did not cover the entire vertical range occupied by six bathypelagic chaetognaths from Table 3.1. Significantly greater night abundances for these species could be interpreted as diel vertical migration as well as visual avoidance behavior. Since none of the species showed significantly greater night abundance, both these hypotheses should be rejected. That is, there was neither diel vertical migration nor visual avoidance of towed nets by these six species.

Analysis of day-night catch differences from published sources is presented in Table 3.2. Gehringer (1962) did not include any data, so it was not possible to determine the extent of the differences or the conditions under which the samples were collected. Tranter et al. (1968) reported highly significant day/night differences in total chaetognath abundance off the southeast coast of Australia. Examination of their data, however, suggests that patchiness is an equally likely explanation of the day-night differences observed (see Different sized net comparisons for a further discussion of their results). While these published sources yielded conflicting results, when considered with the results of the present study, the bulk of the evidence supports the hypothesis that chaetognaths are sampled equally well by day or night.

Different sized net comparisons

As discussed earlier, the larger nets should catch more individuals per unit volume of water filtered than smaller nets for species capable of 
Table 3.2. Day/night differences in chaetognath abundance from published reports. 


\begin{tabular}{|c|c|c|c|c|}
\hline Species & $\begin{array}{l}\text { \# of tows } \\
\text { night, day }\end{array}$ & $\frac{\text { Night abund. }}{\text { Day abund. }}$ & $\begin{array}{l}\text { Equal night and } \\
\text { day abundance }\end{array}$ & Reference \\
\hline & & & & \\
\hline Sagitta spp. & 1,1 & 0.64 & - & $\begin{array}{l}\text { Motoda and } \\
\text { Anraku, } 1954\end{array}$ \\
\hline Sagitta spp. & 2,2 & 0.92 & NS & $\begin{array}{l}\text { Motoda and } \\
\text { Anraku, } 1955\end{array}$ \\
\hline Sagitta spp. & $?$ & $?$ & $\mathrm{p}<0.05$ & $\begin{array}{l}\text { Gehringer, } \\
1962\end{array}$ \\
\hline Chaetognaths & 48,56 & 1.75 & $p<0.001$ & $\begin{array}{l}\text { Tranter et } \\
\text { a1., } 1968\end{array}$ \\
\hline
\end{tabular}


avoidance. Comparisons of the catches of four large chaetognath species by the MOC 1 and MOC 10 produced results opposite to those predicted (Table 3.3). A Wilcoxon signed-rank test applied to the MOC $1 /$ MOC10 sets confirmed that the smaller MOC I caught more than the MOC $10(p<0.01)$. Either the assumptions of the theoretical model are incorrect, or other sources of sampling error were producing the differences seen.

The most important assumptions for this prediction are that escape velocity and reaction distance are independent of net size. There is no reason to suspect that chaetognaths' escape velocity varies with net size. It is possible, however, that that larger nets produce larger signals that are more easily perceived by chaetognaths, thus increasing their reaction distance. A large increase in reaction distance would make it easier for chaetognaths to avoid larger nets than smaller nets. I have examined this hypothesis using Barkley's graphical method of analysis. The analysis suggests that this hypothesis is incorrect, but I will defer the discussion until presentation of the results of the graphical analysis of avoidance.

A more likely hypothesis is that the differences in catch between the MOC 1 and MOC 10 were due to mesh selection. If one accepts the assumption that the width of the chaetognath determines whether or not it will pass through the meshes, then it is clear that the $3.0 \mathrm{~mm}$ mesh of the MOC 10 is too coarse to catch most chaetognaths. Maximum lengths for the species used in this analysis were $41 \mathrm{~mm}$ for $\underline{\mathrm{S}}$. 1yra, $61 \mathrm{~mm}$ for $\underline{\mathrm{s}}$. hexaptera, 41 $\mathrm{mm}$ for S. planctonis, and $49 \mathrm{~mm}$ for S. maxima. The lengths above which these species should be sampled adequately are $39 \mathrm{~mm}, 48 \mathrm{~mm}, 40 \mathrm{~mm}$, and 44 $\mathrm{mm}$, respectively (see the discussion of mesh selection for details of this 
Table 3.3. Comparison of the catch of the MOC 1 and MOC 10 for four chaetognath species. Where two tows are shown together, the numbers $/ \mathrm{m}^{2}$ were averaged. 


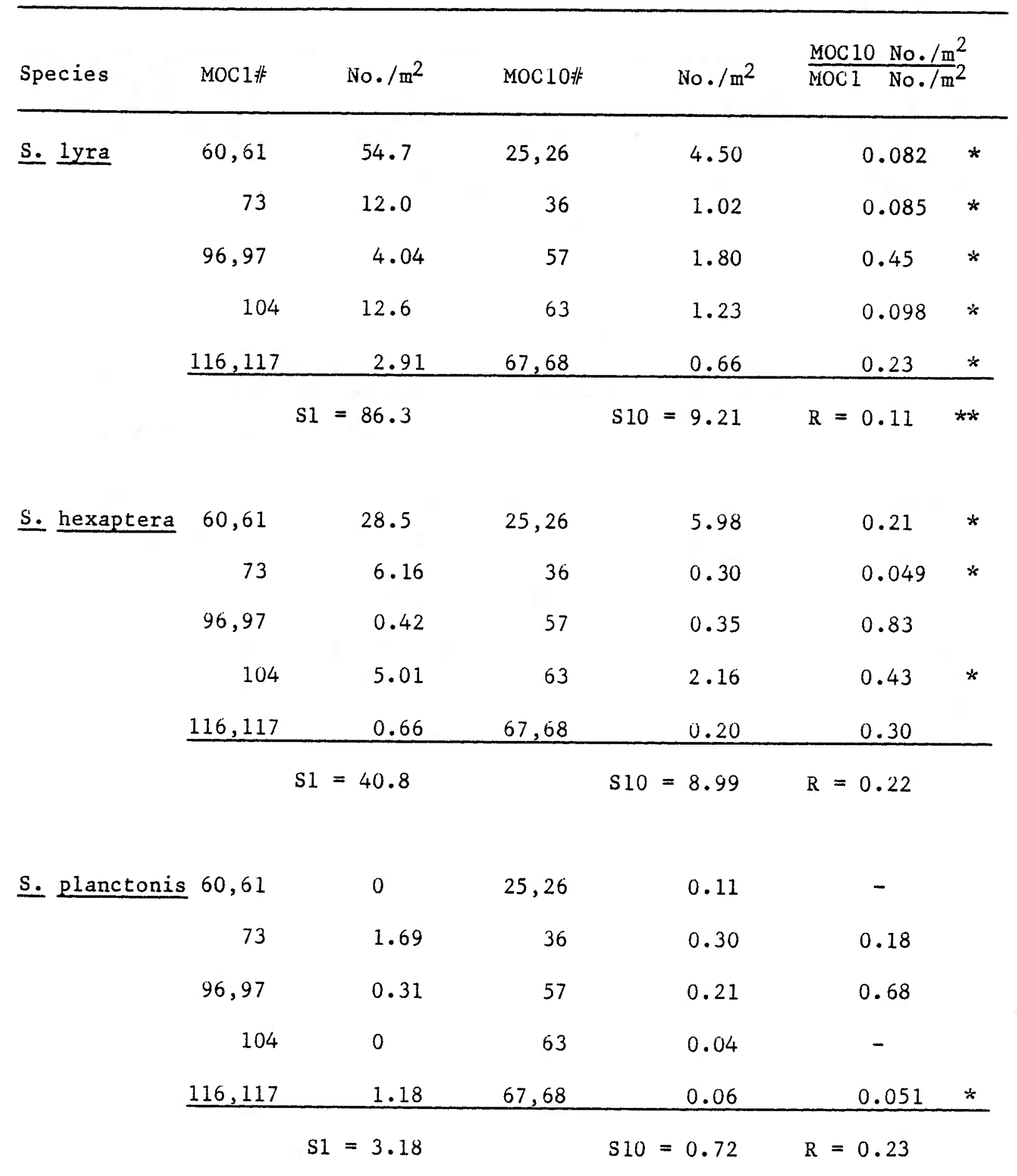


Table 3.3. (cont'd).

\begin{tabular}{|c|c|c|c|c|c|c|c|}
\hline Species & MOC 1 \#⿰三丨⿰⿱三夕⿰丨三丨 & No. $/ m^{2}$ & MOC 10 非 & No. $/ \mathrm{m}^{2}$ & $\frac{\text { MOC } 10}{\text { MOC } 1}$ & $\frac{0 \mathrm{No} \cdot / \mathrm{m}^{2}}{\mathrm{No} \cdot / \mathrm{m}^{2}}$ & \\
\hline \multirow[t]{6}{*}{ S. maxima } & 60,61 & 0 & 25,26 & 0 & & - & \\
\hline & 73 & 1.34 & 36 & 1.77 & & 1.32 & \\
\hline & 96,97 & 1.75 & 57 & 0.22 & & 0.13 & \\
\hline & 104 & 0 & 63 & 0 & & - & \\
\hline & 116,117 & 5.41 & 67,68 & 0.67 & & 0.12 & $*$ \\
\hline & \multicolumn{3}{|c|}{$S 1=8.50$} & $S 10=2.66$ & $\mathrm{R}=\mathrm{C}$ & 0.31 & \\
\hline
\end{tabular}

* Tows for which Spearman rank correlation coefficient was calculated for chaetognath size and MOC $1 /$ MOC 10 ratio (see text).

$* * S 1$ is the sum of the MOC 1 no. $/ \mathrm{m}^{2}, S 10$ is the sum of the MOC 10 no. $/ \mathrm{m}^{2}$, and $\mathrm{R}$ is the ratio $\mathrm{Sio} / \mathrm{S} 1$. 
analysis).

Comparisons of catches of different sized nets from published reports generally support the hypothesis that larger nets catch more individuals per unit volume of water filtered than do smaller nets (Table 3.4). The data of Tranter et al. (1968) provided a striking exception, however. Day hauls with nets of three sizes showed no differences in total chaetognath abundance, suggesting little or no avoidance, but night hauls yielded results opposite those of avoidance theory (Table 3.4 ). I can think of no reason why this pattern should be significant at night and show no differences by day. As Tranter et al. discuss, these results were more likely due to patchiness rather than to net size or time of day. They also did not detect significant differences in total chaetognath abundance for nets towed at different speeds. Avoidance theory predicts larger catches for nets towed at greater speeds, as long as filtration efficiency remains constant. As with net size comparisons, this assumes reaction distance and escape velocity are independent of net speed.

The values of $R$ (Table 3.4) less than one for $\underline{S}$ elegans $(15-21 \mathrm{~mm})$ and E. hamata $(5-10 \mathrm{~mm})$ from Sand's (1978) study were probably due to mesh selection, as $0.5 \mathrm{~mm}$ mesh was used on the small net and $1 \mathrm{~mm}$ mesh was used on the large net (see section on mesh selection).

For values of $\mathrm{R}$ from Table 3.4 greater than one, the corresponding reaction distances calculated from equation (4) are presented in Table 3.5. All the calculations produced relatively small reaction distances $(<$ $35 \mathrm{~cm})$. Also presented in Table 3.5 are calculations of the percent capture ( $P$ x 100 from equation (2)) for individuals of three sizes (assuming $u=10 \mathrm{~L}$ ) under conditions corresponding to the use of the MOC 1 in 
Table 3.4. Ratio ( $R$ ) of the catch of large nets to small nets from published reports. 


\begin{tabular}{|c|c|c|c|}
\hline Species & $\begin{array}{c}\text { Net } \\
\text { diameter }(\mathrm{cm})\end{array}$ & $\mathbf{R}$ & Reference \\
\hline S. elegans, & 75 & $2.42 * *$ & Winsor and \\
\hline \multirow[t]{2}{*}{ Table IV } & 30.5 & $1.89 \mathrm{NS}$ & Clarke, 1940 \\
\hline & 12.7 & - & \\
\hline S. elegans, & 75 & $1.79 *$ & Winsor and \\
\hline Table VIII & 12.7 & - & Clarke, 1940 \\
\hline Chaetognaths, & 113 & $1.00 \mathrm{NS}$ & Tranter et \\
\hline \multirow[t]{2}{*}{ Day } & 56.4 & $1.02 \mathrm{NS}$ & al., 1968 \\
\hline & 25.2 & - & \\
\hline Chaetognaths, & 113 & $0.24 * * *$ & Tranter et \\
\hline \multirow[t]{2}{*}{ Night } & 56.4 & $0.71 * * x$ & a1., 1968 \\
\hline & 25.2 & - & \\
\hline E. hamata, & 113 & $1.00^{1} *$ & Sands, 1978 \\
\hline $5-10 \mathrm{~mm}^{2}$ & 33 & - & \\
\hline E. hamata, & 113 & $1.00 \mathrm{NS}$ & Sands, 1978 \\
\hline $10-25 \mathrm{~mm}$ & 33 & - & \\
\hline
\end{tabular}


Table 3.4. (cont'd).

\begin{tabular}{|c|c|c|c|}
\hline Species & $\begin{array}{c}\text { Net } \\
\text { diameter }(\mathrm{cm})\end{array}$ & $\mathrm{R}$ & Reference \\
\hline E. hamata, & 113 & $1.81 *$ & Sands, 1978 \\
\hline $25-30 \mathrm{~mm}$ & 33 & - & \\
\hline E. hamata, & 113 & $2.03 *$ & Sands, 1978 \\
\hline $30-35 \mathrm{~mm}$ & 33 & - & \\
\hline \multicolumn{4}{|l|}{ E. } \\
\hline bathypelagica, & 113 & $1.00 \mathrm{NS}$ & Sands, 1978 \\
\hline $21-33 \mathrm{~mm}$ & 33 & - & \\
\hline S. elegans, & 113 & $1.00^{1} *$ & Sands, 1978 \\
\hline $15-21 \mathrm{~mm}$ & 33 & - & \\
\hline S. elegans, & 113 & $1.20 *$ & Sands, 1978 \\
\hline $21-24 \mathrm{~mm}$ & 33 & - & \\
\hline
\end{tabular}

The probability that the large and small nets catch equal numbers was either supplied by the author cited or determined from a t-test on the $\log _{10}(X+1)$ transformed abundances $(*=p<0.05, * *=p<0.01, * * * *=p<0.001$, NS= not significant).

1 These results were most likely due to mesh selection. 2 These values were for individual length. 
Table 3.5. Calculated reaction distances and percent capture for individuals of three sizes from data in Table 3.4 . 


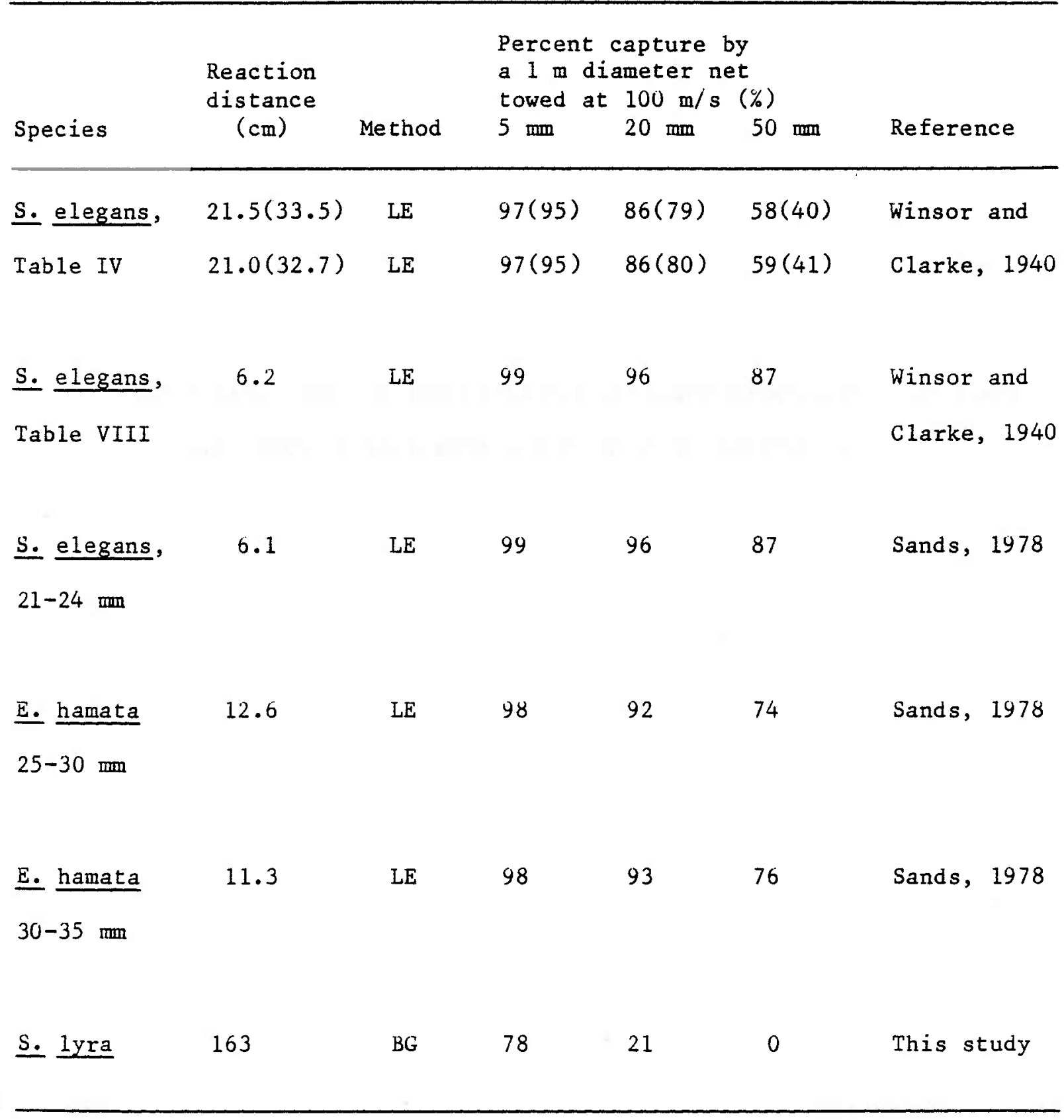

The reaction distances were calculated by one of two methods- either from the equations provided by Laval (1974) (LE), or by Barkley's graphical method $(B G)$. The two values for $S$. elegans from Table IV of Winsor and Clarke (1940) result from assuming average sizes of $15 \mathrm{~mm}$ and (10 mm). 
the present study. These results suggest that only the larger individuals $(>20 \mathrm{~mm})$ were seriously undersampled as a result of avoidance.

\section{Graphical analysis of avoidance}

Of the four species analyzed by the graphical method, only the $\underline{S}$. lyra data corresponded well enough with the graphical predictions to estimate reaction distance. The size frequencies for $\underline{S}$. hexaptera, $\underline{\text { S. maxima }}$ and $\underline{\text { s. }}$ planctonis did not match any of the $x / R$ isolines very well. Typical comparisons of the size frequencies for these four species are shown in Figure 3.1. The results do not necessarily mean that there was no avoidance by the latter three species- it may mean that the true population size structure obscured any avoidance which is detectable by this method.

The average value of $x / R$ from ten comparisons for $S$. 1yra from the NSS was 3.3 ( \pm 0.21 std. dev.). Three size frequency curves for $\underline{S}$ lyra from the SW did not correspond well with any of the $x / R$ isolines. NSS stations for S. lyra are denoted by triangles and SW stations by circles in Figure 3.1. Since the net radius of the $M O C 1$ can be approximated as $50 \mathrm{~cm}$, the reaction distance was $163 \mathrm{~cm}$, which was much greater than the reaction distances determined from the net size comparisons and results in a considerably larger estimate of avoidance (Table 3.5).

One must be cautious in applying the results of the graphical analysis, however, as the assumption of equal numbers in all size classes is rarely found in nature. Most aquatic invertebrates show a decrease in abundance with increasing size. The size frequency of $\underline{S}$. Iyra from our samples may 
Figure 3.1. Graphical analysis of avoidance. The values along the right and bottom margins of the graphs are values of the $x / R$ isolines. Where two tows were available from the same location, size frequency curves were averaged to reduce the effects of patchiness. 

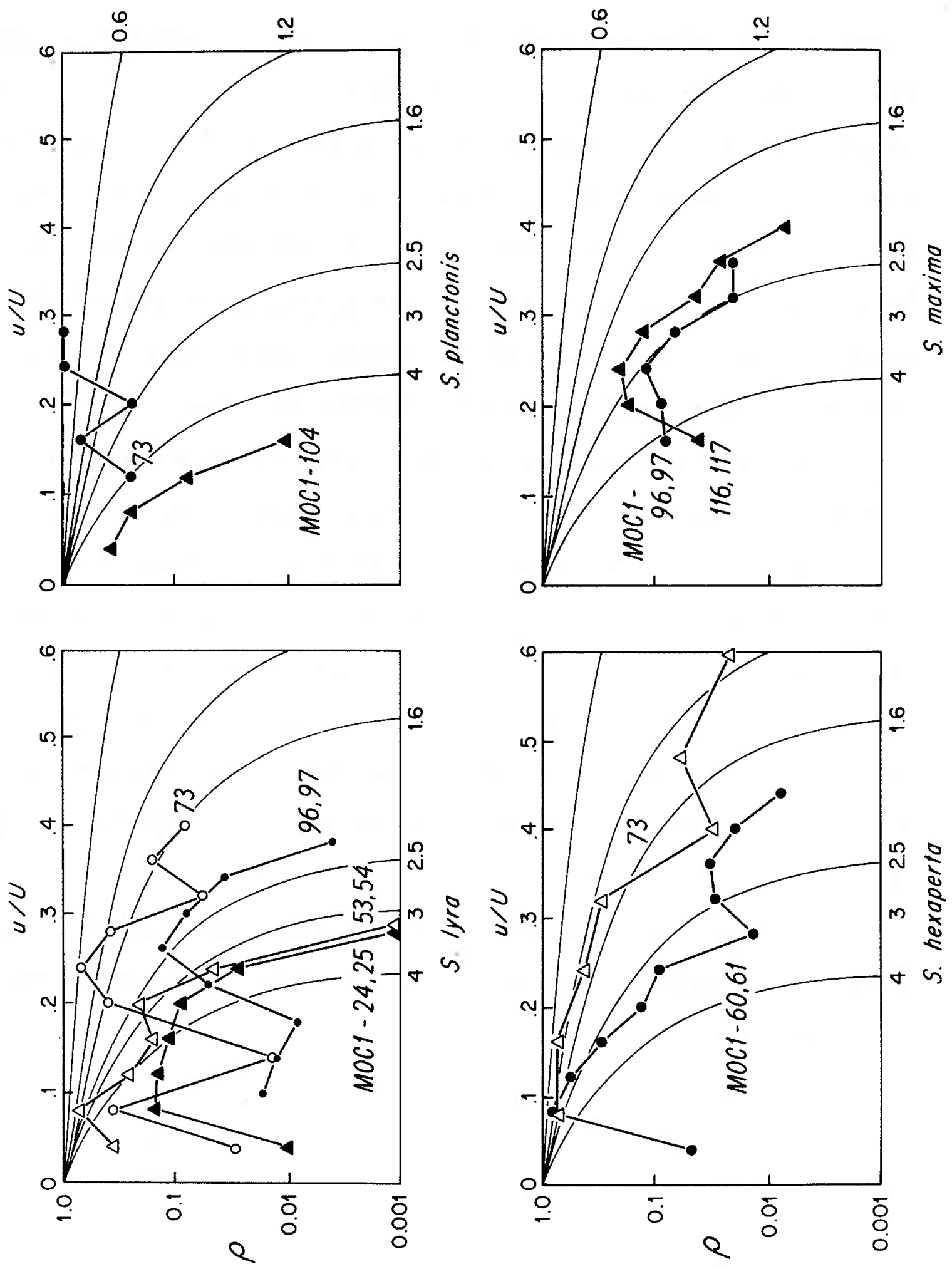
be the result of either true population size structure or avoidance or some combination of the two. As can be seen from Figure 3.1, the correspondence between size frequency and the $x / R$ isolines for $\underline{S}$. Iyra from the NSS was primarily for $u / U$ values greater than 0.15 (15 body length). The lack of correspondence for values less than 0.15 and for the three SW size frequency curves implies that true population size structure was responsible for at least some of the observed size frequency pattern. The results of the graphical analysis for $\underline{S}$. lyra are probably best interpreted as an upper limit to its avoidance capabilites, with the true reaction distance being somewhere between 163 and $0 \mathrm{~cm}$.

As discussed earlier, the graphical analysis suggests a means to examine the hypothesis that the MOC $1 / M O C$ lO catch differences were due to an increased reaction distance for the larger net. Examination of the graph in Figure 3.1 shows that the $x / R$ isolines diverge as $u / U$ increases. Since the probability of capture $(P)$ is plotted on a logarithmic scale, an equivalent observation is that the ratio of $P$ values for different values of $x / R$ and equal values of $u / U$ should increase with increasing value of $\mathrm{u} / \mathrm{U}$ (when $\mathrm{P}$ for the smaller $\mathrm{x} / \mathrm{R}$ isoline is divided by $\mathrm{P}$ for the larger $\mathrm{x} / \mathrm{R}$ value).

An example will serve to illustrate this point. The ratio of $P^{\prime} s$ from Figure 3.1 for values of $x / R$ of 1.6 and 2.5 and the corresponding values of $\mathrm{u} / \mathrm{U}$ are as follows:
$\mathrm{u} / \mathrm{U}$
0.10
0.15
0.20
0.25
0.30
0.35
$P(1.6) / P(2.5) \quad 1.1$
1.2
1.3
1.5
1.8
2.6 
Since the probability of capture for any particular chaetognath size was observed to be greater for the MOC 1 than the MOC 10 , the $x / R$ isoline for the MOC 10 must have had a greater value than that for the MOC 1. The ratio of the $P$ values is equivalent to the ratio of the catches of the two nets $\left(P_{i}=\right.$ abundance per $\mathrm{m}^{2}$ in net $i /$ true abundance, so that $P_{1} / P_{2}=$ abundance per $\mathrm{m}^{2}$ in net $1 /$ abundance per $\mathrm{m}^{2}$ in net 2 ). Since $u / U$ is a function of chaetognath size ( $u=10 \mathrm{~L})$, we would expect a Spearman rank correlation coefficient of 1.0 for the relationship between the ratio of MOC 1 to MOC 10 abundance $\left(=P_{1} / P_{2}\right)$ and chaetognath length $(=100 \mathrm{u} / \mathrm{U})$, if in fact the differences in catch between the two nets were due to an increased reaction distance for the larger net. I have calculated the correlation coefficients for ten of the MOC $1 /$ MOC 10 sets (Table 3.3). Values of the correlation coefficient ranged from -0.77 to +0.63 with an average value of +0.04 . Although this analysis suggests that an increase in reaction distance for the MOC 10 was not responsible for the differences seen, it is far from conclusive proof. It is interesting to note that mesh selection theory predicts that the catch ratio of the large mesh net (MOC 10) to the small mesh net (MOC 1) should increase to a value of one as chaetognath size increases. The observed independence of chaetognath size and MOC $1 /$ MOC 10 catch ratio could be due to the opposing influences of avoidance and mesh selection, or it could be due to variability in the catches per size class for these two nets which might obscure any relationship of chaetognath size and catch ratio. 
Discussion

The results of this study suggest that avoidance of the MOC 1 by chaetognaths was not a major source of sampling error, except for the larger individuals of the larger species. A large part of this analysis depends upon assumptions made about the escape velocity and angle of flight of the organisms in question. Laboratory experiments are needed to determine escape velocity and its dependence on organism size and to determine the angle of flight from a disturbance in the water. Experiments are also needed to determine the types of stimuli produced by nets which can be perceived by chaetognaths.

In addition, further field experiments can provide a better quantification of the avoidance capabilities of chaetognaths. Lava1 (1974) outlined a field experiment whereby the parameters of equation (2) can be estimated. Two nets of different diameter, $D_{1}$ and $D_{2}$, are first towed at speed $U_{1}$ and then at speed $U_{2}$. Several such series would be needed to average the patchiness inherent in sampling plankton. The ratio of the catches per unit volume of water filtered for nets $D_{1}$ and $D_{2}$ can then be used to calculate $2 \mathrm{xV}_{1}$ and $2 \mathrm{xV}_{2}$ from equation (4). The ratio $2 \mathrm{xV}_{1} / 2 \mathrm{xV}_{2}(=\mathrm{d})$ can be used to calculate ucos $\theta$ as follows:

$\mathrm{u} \cdot \cos \theta=\left(\mathrm{d} \cdot \mathrm{U}_{1}-\mathrm{U}_{2}\right) /(\mathrm{d}-\mathrm{I}) \cdot$

(5) Laval (11)

ucos $\theta$ can now be used to calculate $2 x u S I N \theta$ from either $2 \mathrm{xV}_{1}$ or $2 \mathrm{xV}_{2}$. Given $u \cos \theta$ and $2 x u S I N \theta$, we can determine the probability of capture (P) from equation (2) for a net of any diameter towed at any speed. If we have 
an independent measure of the escape velocity, as from laboratory experiments, then the reaction distance and angle of flight can be calculated from these equations.

If we wish to reduce the sampling bias resulting from avoidance, we can proceed in two ways. The first is to determine the parameters of equation (2) as discussed above, and then calculate what combination of net size and towing speed is necessary to reduce or eliminate avoidance. There are, however, constraints on both the size of nets and the speed at which they can be towed (Barkley, 1964). A second approach is to determine what cues are produced by the net which the organisms are sensing. We can reduce these stimuli, thus reducing the reaction distance, and/or we can overwhelm the organisms with stimuli such that they become disoriented, as has recently been suggested by wiebe et al (1982). An example of the effectiveness of a reduction in stimulus is that sampling with dark colored nets reduces visual avoidance and results in larger catches (LeBrasseur and McAllister, cited by Clutter and Anraku, 1968). For organisms which detect displacements of water in front of the net, removing bridles and cables from the front of the net, streamlining the net frame and attached instrumentation, and designing nets such that filtration efficiency remains high throughout the duration of a tow should reduce avoidance.

How might chaetognaths detect towed nets? The lack of day/night differences in abundance as sampled by nets supports the hypothesis that chaetognaths do not visually avoid nets. It may be argued, however, that visual cues produced by bioluminescence in the net at night may result in equal visually oriented avoidance during the day and the night. Recent 
studies suggest that some copepods may visually detect prey or avoid predation (Gophen and Harris, 1981; Singarajah, 1975). Visual cues are probably limited to a distance of several centimeters, however. Chaetognaths do not have image-forming eyes (Eakin and Westfal1, 1964) and they do not appear to use vision for prey capture (Reeve, 1964; Feigenbaum, 1977). It appears unlikely that chaetognaths visually detect nets more than a few centimeters away.

A more likely cue for the detection of nets by chaetognaths is the displacement of water in front of the net. Both cyclic (from wire struming) and noncyclic water displacements may precede nets and are detectable up to $1.5 \mathrm{~m}$ ahead of the net by flowmeters (Smith, cited by Clutter and Anraku, 1968). The chaetognaths Spadella cephaloptera, Sp. schizoptera, Sagitta hispida, and S. euneritica are known to detect prey by vibrations produced by the swimming and feeding motions of their prey (Horridge and Boulton, 1967; Feigenbaum and Reeve, 1977). Sensory hairs, presumably for the detection of water movements produced by predators and prey, are arranged on the bodies of chaetognaths (Horridge and Boulton, 1967; Feigenbaum, 1978) and may allow them to detect water displacements preceding towed nets.

It is not known if chaetognaths can detect sound produced by cables and nets, but no structures resembling sound receptors have been identified from them.

At present, it appears that reducing the displacement of water in front of towed nets by removing bridles and cables and streamlining the net frame and associated instrumentation is the most reasonable approach for reducing avoidance by chaetognaths. Determination of optimum net size and 
towing speed will have to await a better understanding of the parameters necessary to predict avoidance capabilities of chaetognaths from equation (2). This study suggests, however, that a $1 \mathrm{~m}$ diameter net towed at $1 \mathrm{~m} / \mathrm{s}$ ( 2 knots) should catch most $(>80 \%$ ) chaetognaths less than $20 \mathrm{~mm}$ in length. 
C. Mesh selection

Introduction

Many planktonic organisms are small enough to pass through the meshes of plankton nets. This phenomenon of retention of the larger organisms and loss of many of the smaller organisms, known as mesh selection, results in an undersampling of the smaller plankters. Whether or not an individual is retained by the net depends upon upon the mesh size of the net, the size, shape, flexibility, and activity of the organism, the pressure drop across the meshes, and aspects of the net material such as the type of material, the nature of the weave, the flexibility of the strands, and variability in the sizes of the mesh openings (Saville, 1968; Vannucci, 1968).

The most important variables are the mesh size and the size of the organisms being sampled. Very little information is available to assess mesh selection of chaetognaths by mesh of different sizes.

Given a mesh size, the width of the organism appears to be the most important factor determining its retention, although flexibility of both the organism and the mesh strands and variability in mesh size can result in loss of organisms somewhat larger than the mean mesh width (Saville, 1968; Omori et al., 1965; Heron, 1968). Length is typically used to characterize the size of planktonic organisms, so I have calculated length versus width regressions for fourteen chaetognath species from the MOCNESS samples. Given mesh size, these regressions can be used to calculate the minimum lengths of chaetognaths caught efficiently by that mesh size. Although not a statistically advisable procedure (because it involves 
extrapolation of the regression equations beyond the data), the mesh size necessary to sample the entire size range for any of these species can be estimated if the length of newly hatched individuals is known.

Comparisons of size frequencies caught by two nets of $0.333 \mathrm{~mm}$ and 3.0 $\mathrm{mm}$ mesh reported here and data from published reports suggests that the $100 \%$ retention sizes are larger than predicted from the regressions. These observations are probably due to several factors discussed above, mesh and organism flexibility and variation in mesh size, but may also be due in part to problems in measuring the widths of the chaetognaths in this analysis. In spite of their shortcomings, these regressions provide useful estimates of chaetognath sizes which are sampled effectively by mesh of different sizes.

Methods

Zooplankton samples were collected on six cruises in the western North Atlantic Ocean during the period August 1975 to November 1977 using the MOC $1(0.333 \mathrm{~mm}$ mesh) and the MOC 10 (3 mm mesh). Details of the sampling gear and sampling procedures can be found in Chapter 2. Samples were preserved in $5-10 \%$ formalin.

Chaetognaths were removed from the samples or aliquots of the samples and identified to species. Usually, between 50 and 101 individuals were used in the length versus width analysis. Individuals were selected from five to ten different tows so as to include spatial and temporal variability and possible differences in preservation and handing 
techniques.

Lengths and widths were measured with a calibrated ocular micrometer on a Wild dissecting microscope. Length was measured from the tip of the head to the end of the tail section, excluding the caudal fin. Width, or more accurately body diameter, was measured across the widest portion of the body, excluding the lateral fins. For most of these species the width measurement was made near the middle of the body, but for some species measurements were made of the width of the head.

Many of the preserved specimens were somewhat dorso-ventrally flattened instead of maintaining the cylindrical shape of live specimens. I attempted to select specimens with minimum dorso-ventral flattening, but as a result of this flattening, predictions of width from these regressions may overestimate the width for a particular length of live individuals. This would result in a predicted minimum retention length smaller than what actually occurs. Head width measured for some of these species was highly variable, depending upon whether the grasping hooks were expanded or contracted during preservation. Some shrinkage of individuals occurs with formalin preservation, but I did not attempt to account for this. Sands (1980) reported about a 5\% reduction in length for E. hamata, but it is not clear whether or not body width changes after preservation.

Geometric mean regressions were calculated for the $\log _{10}$ transforms of length and width. $\log _{10}$ transforms were used to reduce the dependence of the variance on the mean typical of many morphometric measurements (Teissier, 1948). It also was not clear that width should remain a constant proportion of length over the entire range of lengths for each of 
these species, so the $\log _{10}$ transforms should provide a better description of the relationship between length and width if in fact width is not a constant proportion of length.

Geometric mean regressions were determined to be the appropriate regressions for these data for reasons discussed by Ricker (1973). The length and width measurements were not normally distributed, as I attempted to measure equal numbers of individuals from a large size range. It was also desirable to have a regression equation from which either length or width could be predicted, and because it may be necessary to extrapolate beyond the limits of these data, the geometric mean regression was the most appropriate regression for the data at hand.

Results

Table 3.6 presents the geometric mean regressions for fourteen chaetognath species. In general, the correlation coefficients were large, but three values below 0.8 were found for regressions of length and head width, the variability of which was noted earlier. For these three species, individuals with both expanded and contracted grasping spines were measured. Two other species for which head width measurements were taken, S. macrocephala and S. planctonis, had large correlation coefficients. For these two species, only specimens with contracted hooks were measured.

An interesting feature of these regression equations is that the slope of the $\log _{10}$ transformed data is less than 1.00 for fifteen of the 
Table 3.6. Geometric mean regressions of length and width. 


\begin{tabular}{|c|c|c|c|c|c|}
\hline Species & $\begin{array}{l}\text { Sample } \\
\text { size }\end{array}$ & $\begin{array}{l}\text { Correlation } \\
\text { coefficient }\end{array}$ & Regres & sion equation & \\
\hline E. hamata & 51 & 0.960 & $\log (L)$ & $=0.894 \log (W)$ & +1.1465 \\
\hline K. pacifica & 32 & 0.881 & $\log (L)$ & $=0.8561 \log (W)$ & +1.1034 \\
\hline K. pacirica $^{1}$ & 31 & 0.514 & $\log (L)$ & $=0.9652 \log (W)$ & +1.0867 \\
\hline K. subtilis & 101 & 0.955 & $\log (I)$ & $=0.9134 \log (W)$ & +1.3277 \\
\hline K. subtilis ${ }^{1}$ & 100 & 0.715 & $\log (L)$ & $=1.4152 \log (W)$ & +1.2766 \\
\hline P. draco $^{1}$ & 100 & 0.581 & $\log (L)$ & $=0.4689 \log (W)$ & +0.8065 \\
\hline S. bipunctata & 64 & 0.948 & $\log (L)$ & $=0.806110 g(W)$ & +1.1103 \\
\hline S. decipiens & 101 & 0.896 & $\log (L)$ & $=0.9243 \log (\mathrm{W})$ & +1.1570 \\
\hline S. hexaptera & 101 & 0.975 & $\log (L)$ & $=0.9368 \log (W)$ & +1.1399 \\
\hline S. Iyra & 101 & 0.972 & $\log (L)$ & $=0.8786 \log (W)$ & +1.0887 \\
\hline S. macrocephala ${ }^{1}$ & 51 & 0.950 & $\log (L)$ & $=0.9993 \log (W)$ & +1.0395 \\
\hline S. maxima & 51 & 0.951 & $\log (L)$ & $=0.8906 \log (W)$ & +1.0892 \\
\hline S. minima & 62 & 0.901 & $\log (L)$ & $=0.7903 \log (W)$ & +1.0328 \\
\hline S. planctonis & 66 & 0.875 & $\log (L)$ & $=0.6868 \log (W)$ & +1.0769 \\
\hline S. planctonis & 66 & 0.913 & $\log (L)$ & $=1.0432 \log (W)$ & +0.9909 \\
\hline S. serratodentata & 101 & 0.963 & $\log (L)$ & $=0.7758 \log (W)$ & +1.1372 \\
\hline S. tasmanica & 50 & 0.943 & $\log (\mathrm{L})$ & $=0.6883 \log (\mathrm{W})$ & +1.1702 \\
\hline
\end{tabular}

1 These regressions were of head width and body length. All other regressions were for body width and body length. 
Table 3.6. (cont'd).

\begin{tabular}{|c|c|c|c|c|c|c|}
\hline Sp. & $s^{2}$ & $\overline{\log (W)}$ & $w^{2}$ & $s^{2}$ & $\overline{\log (L)}$ & $1^{2}$ \\
\hline E.h. & 0.002633 & 0.0451 & 2.0384 & 0.003292 & 1.1868 & 1.6294 \\
\hline $\mathrm{K} \cdot \mathrm{p}$. & 0.002113 & -0.4514 & 0.3867 & 0.002878 & 0.7169 & 0.2838 \\
\hline$k \cdot p$. & 0.007185 & -0.3830 & 0.3042 & 0.007715 & 0.7170 & 0.2833 \\
\hline K.s. & 0.002194 & -0.4063 & 2.9660 & 0.002630 & 0.9565 & 2.4745 \\
\hline K.s. & 0.01216 & -0.2275 & 1.2166 & 0.006070 & 0.9546 & 2.4369 \\
\hline P.d. & 0.008769 & -0.2019 & 5.9053 & 0.03989 & 0.7119 & 1.2982 \\
\hline S.b. & 0.001766 & -0.3959 & 1.6605 & 0.002718 & 0.7912 & 1.0788 \\
\hline S.d. & 0.003161 & -0.2532 & 1.8525 & 0.003700 & 0.9230 & 1.5826 \\
\hline S.h. & 0.002383 & 0.0080 & 5.4445 & 0.002715 & 1.1474 & 4.7786 \\
\hline S. 1 . & 0.001641 & 0.0930 & 3.7727 & 0.002126 & 1.1704 & 2.9119 \\
\hline S.m. & 0.002099 & -0.0243 & 1.0547 & 0.002103 & 1.0152 & 1.0529 \\
\hline S.m. & 0.003600 & 0.1246 & 2.3353 & 0.004538 & 1.2001 & 1.8524 \\
\hline S.m. & 0.001514 & -0.4361 & 0.7692 & 0.002424 & 0.6882 & 0.4803 \\
\hline S.p. & 0.009549 & -0.0639 & 5.5328 & 0.02025 & 1.0331 & 2.6095 \\
\hline$S \cdot p \cdot$ & 0.006793 & 0.0404 & 2.3979 & 0.006243 & 1.0331 & 2.6095 \\
\hline S.s. & 0.0009048 & -0.4264 & 2.0275 & 0.001503 & 0.8064 & 1.2204 \\
\hline S.t. & 0.002023 & -0.3911 & 1.8348 & 0.004269 & 0.9010 & 0.8692 \\
\hline
\end{tabular}


seventeen regressions. If these equations are converted to their untransformed counterparts $\left(L=b W^{a}\right)$, it can be seen that for values of the slope (a) less than 1.00, width is not a constant proportion of length, but in fact is a decreasing proportion of length as length increases. In other words, shorter chaetognaths are broader in relation to length than longer chaetognaths.

Also included in Table 3.6 are several quantities necessary to predict confidence limits for these regression equations. Although not strictly applicable to the geometric mean regression, standard errors for the $Y$ on $X$ regression can be used to calculate confidence limits for the geometric mean regression. Ricker (1973) stated that these confidence limits "...are a reasonable approximation to the true limits and will rarely lead to incorrect conclusions."

The variance $\left(s^{2}\right)$ of a single estimate of length from the regression equation is given by

$s^{2}=\left(s_{L}^{2}\left(1-r^{2}\right)\right)\left(1+1 / N+(\log W-\overline{\log W})^{2} / w^{2}\right)$

where $s_{L}^{2}$ is the variance of length, $r$ is the correlation coefficient, $\mathrm{N}$ is the sample size upon which the regression is based, $\log \mathrm{W}$ is the $\log _{10}$ transform of the width for which length is to be predicted, $\overline{\operatorname{logW}}$ is the mean $\log _{10}$ transform of width from the regression data, and $w^{2}$ is the sum of the squared deviations of the $\log _{10}$ transformed width data.

A similar equation for the variance of a width estimate from the regression equation is given by: 


$$
s^{2}=\left(s_{W}^{2}\left(1-r^{2}\right)\right)\left(1+1 / N+(\log L-\overline{\log L})^{2} / 1^{2}\right)
$$

where $s_{W}^{2}$ is the variance of width, $r$ is again the correlation coefficient, $\mathbf{N}$ is the sample size upon which the regression is based, $\log L$ is the $\log _{10}$ transform of the length for which width is to be predicted, $\overline{\log L}$ is the mean $\log _{10}$ transform of length from the regression data, and $1^{2}$ is the sum of the squared deviations of the $\log _{10}$ transformed length data.

These regression equations were used to calculate minimum lengths for the fourteen chaetognath species as sampled by the MOC $1(0.333 \mathrm{~mm}$ mesh) and the MOC 10 ( $3 \mathrm{~mm}$ mesh) (Table 3.7). Lengths calculated from these regressions represent mean lengths for these widths, so to better estimate the size of $100 \%$ retention, one may also calculate the mean length plus the upper $95 \%$ confidence confidence limit for a single prediction. At this length, $97.5 \%$ of the individuals should have widths greater than or equal to the mesh width.

The largest individual length recorded for each species from this study is also presented in Table 3.7 for comparison. For K. pacifica, P. draco

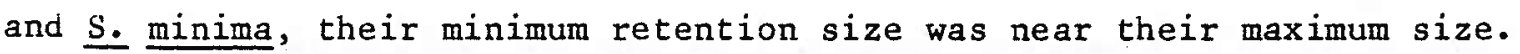
This indicates that the MOCNESS abundance estimates may not represent the total population abundances for these three species.

In the avoidance section, MOC $10 / \mathrm{MOC} 1$ catch ratio was found to be independent of the lengths of S. 1yra, S. maxima, S. planctonis and $\underline{E}$. hamata. Mesh selection theory predicts, however, that the MOC $10 /$ MOC 1 catch ratio should increase from zero to one as chaetognath size increases to the minimum retention length for the $3 \mathrm{~mm}$ mesh of the MOC 10 . It was 
Table 3.7 . Minimum retention lengths for chaetognaths caught by the MOC 1 and MOC 10 as predicted from the regression equations. 


\begin{tabular}{|c|c|c|c|c|c|}
\hline Species & $\begin{array}{l}\text { MOC } \\
\text { Mean }\end{array}$ & $\begin{array}{l}1 \text { length (mm) } \\
\text { tupper } 95 \% \text { c. } 1\end{array}$ & $\begin{array}{l}\text { MOC } \\
\text { Mean }\end{array}$ & $\begin{array}{l}10 \text { length }(\mathrm{mm}) \\
\text { tupper } 95 \% \mathrm{c} .1\end{array}$ & $\begin{array}{l}\text { Maximum } \\
\text { length } \\
(\mathrm{mm})\end{array}$ \\
\hline E. hamata & 5.24 & 6.76 & 37.4 & 48.0 & 30 \\
\hline K. pacifica & 4.95 & 6.14 & - & - & 7 \\
\hline K. pacifica ${ }^{1}$ & 4.22 & 6.32 & - & - & \\
\hline K. subtilis & 7.79 & 9.66 & - & - & 15 \\
\hline K. subtilis ${ }^{1}$ & 3.99 & 0.73 & - & - & \\
\hline P. draco $^{1}$ & 3.82 & 5.92 & - & - & 8 \\
\hline S. bipunctata & 5.31 & 6.46 & - & - & 16 \\
\hline S. decipiens & 5.20 & 6.76 & - & - & 13 \\
\hline S. hexaptera & 4.93 & 6.20 & 38.6 & 48.6 & 61 \\
\hline S. 1yra & 4.67 & 5.67 & 32.2 & 39.0 & 41 \\
\hline S. macrocephala ${ }^{1}$ & 3.65 & 4.61 & - & - & 21 \\
\hline S. maxima & 4.61 & 6.22 & 32.7 & 43.5 & 49 \\
\hline S. minima & 4.52 & 5.42 & - & - & 8 \\
\hline S. planctonis & 5.61 & 8.89 & $25 \cdot 4$ & 40.4 & 41 \\
\hline S. planctonis ${ }^{1}$ & 3.11 & 4.65 & 30.8 & 45.8 & \\
\hline S. serratodentata & 5.84 & 6.72 & - & - & 10 \\
\hline S. tasmanica & 6.94 & 8.56 & - & - & 15 \\
\hline
\end{tabular}

1 These were regressions of head width and body length. All other regressions were for body width and body length. 
speculated that the independence of catch ratio and length was due either to the opposing effects of avoidance and mesh selection or to large variability in the abundance estimates for size classes. In order to reduce the effects of this variability, it was desirable to average the abundances of size classes for several tows in order to assess the effects of mesh selection on the MOC 10 catches.

For the four species for which length was measured from the five MOC 10/MOC 1 tow sets (Table 2.2), the abundance within two millimeter length classes was calculated. If there were two MOC 1 or MOC 10 tows within a set, the average abundance for each size class was calculated, and then the abundance values for each size class were averaged for the five MOC 10 and MOC 1 sets. Assuming that the MOCl catches represent the true abundance of each size class, the percent capture for each size class from the MOC 10 relative to the MOC 1 tows should increase with increasing chaetognath length to a value of $100 \%$ at the minimum retention length (mean plus upper $95 \%$ confidence limit) from Table 3.7 .

It is clear from Table 3.8 that the regressions do a very poor job of predicting the $100 \%$ retention sizes for these four species. For S. 1yra and $\underline{S}$. hexaptera, none of the size classes appear to be sampled well by the $3 \mathrm{~mm}$ mesh of the MOC10. As discussed in the previous section on avoidance, this may be the result of the opposing influences of avoidance and mesh selection. Both S. maxima and S. planctonis appear to reach $100 \%$ retention at sizes smaller than predicted from the regression equations. The lack of predictability of the $100 \%$ retention length may still be due to the variability in capture of the larger size classes even after averaging the abundance values from these tows. For these four species, 
Table 3.8. Comparisons of observed and predicted retention lengths for four chaetognath species from the MOC 10. Mean length and mean plus upper $95 \%$ confidence limit from Table 3.7 are underlined for each species. 


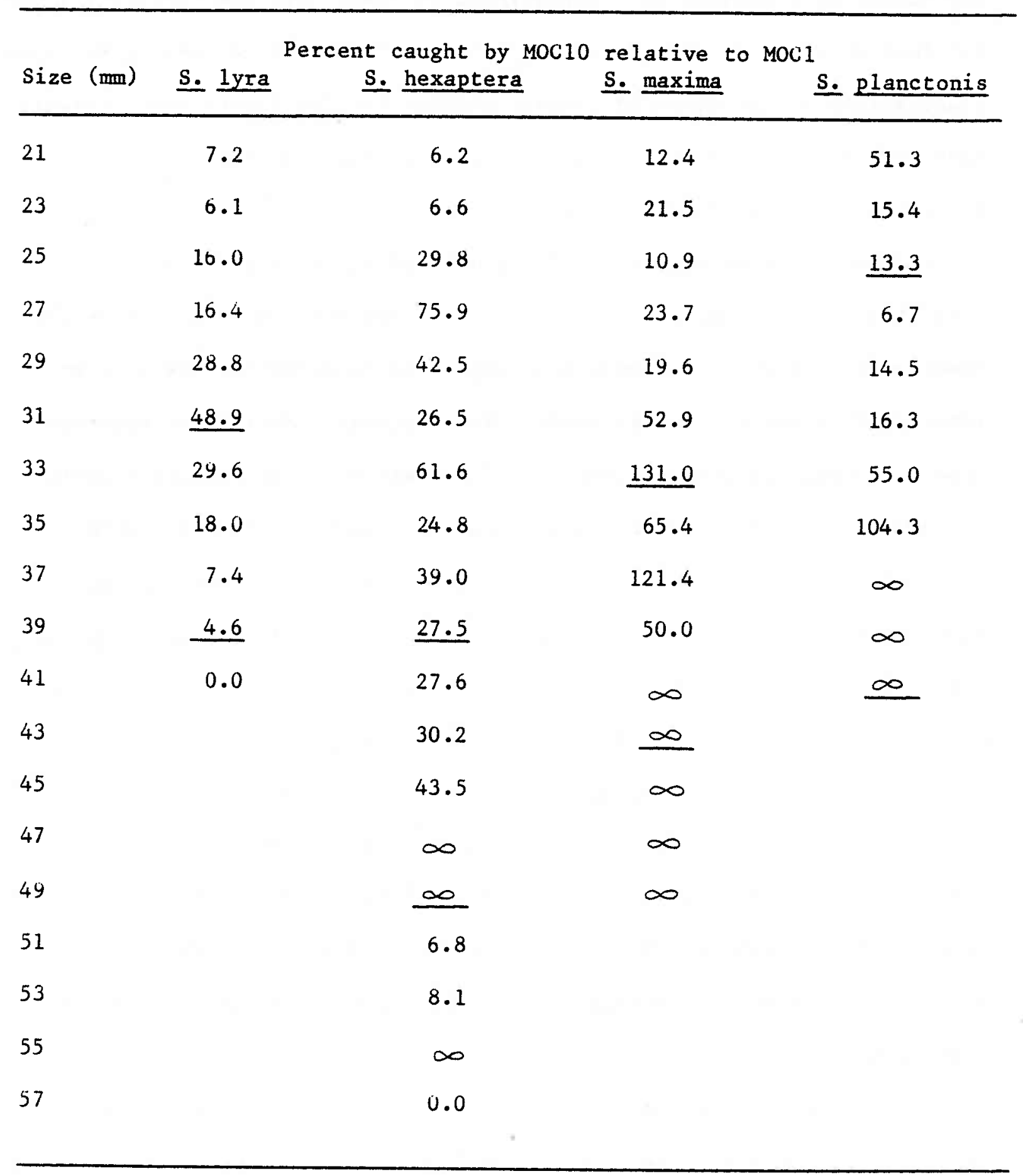

Values of infinity occur when the MOC 1 catch is zero and the MOC $10 \mathrm{catch}$ is greater than zero. 
the larger size classes were represented by fewer than ten individuals for all tows combined in this analysis. Random variability may result in large fluctuations in the computed percent capture for the larger size classes. Many more tows are necessary to accurately assess the length of $100 \%$ retention for these four species.

Data presented by Clarke et al. (1940) and Omori et al. (1965) indicated that mesh sizes of 1.7 and $2.0 \mathrm{~mm}$ seriously undersample smaller chaetognaths. They do not provide enough data to determine the size at which $100 \%$ retention occurs. Sands (1978) presented data which suggests that $\mathrm{S}$. elegans is undersampled by $1.0 \mathrm{~mm}$ mesh until it reaches a length of about $20 \mathrm{~mm}$ and $\mathrm{E}$. hamata is undersampled until it reaches a length of about $10 \mathrm{~mm}$. The predicted retention length from the regressions (mean plus upper $95 \%$ confidence limit) for $1.0 \mathrm{~mm}$ mesh is $18.7 \mathrm{~mm}$ for s. elegans and $17.8 \mathrm{~mm}$ for $\mathrm{E}$. hamata. Since there was no regression for S. elegans, I used the equation for a closely related species, $\underline{S}$. decipiens. The prediction is good for $\underline{S}$ elegans but poor for E. hamata.

Although the available data do not very well support the hypothesis that it is body width which determines the $100 \%$ retention length for chaetognaths, these regressions provide the only means of estimating the lengths of chaetognaths sampled effectively by mesh of various sizes at this time.

These equations may also be used to calculate the mesh size necessary to catch individuals above a specified length. Table 3.9 provides measurements of the lengths of newly hatched larvae and egg diameters of several chaetognath species. One must be cautious when extrapolating beyond the limits of the data used to produce the regression equation, but 
Table 3.9. Published reports of the size of chaetognath eggs and larvae. 


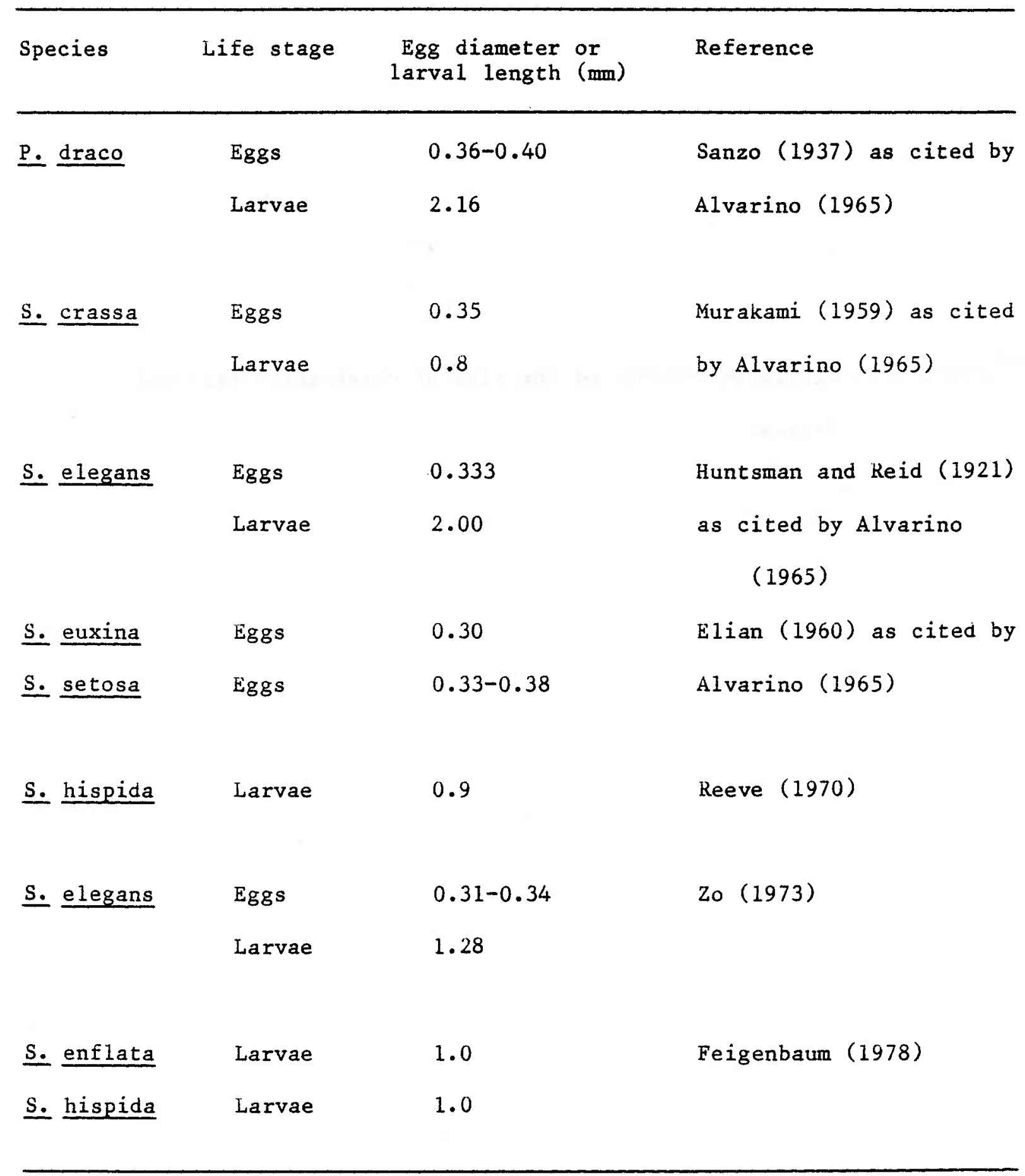


such an extrapolation is at present the only means of determining the appropriate mesh size for collecting all size classes of a particular species. From Table 3.9, we see that minimum larval lengths are about 1 $\mathrm{mm}$, which corresponds to widths from the regressions ranging from 0.019 to $0.125 \mathrm{~mm}$ (these are mean widths and do not include lower $95 \%$ confidence limits). It is clear that a very fine mesh net is needed to sample the entire size range for each species.

\section{Discussion}

The regression equations presented here provide a basis for estimating the $100 \%$ retention lengths for any mesh size for each of these species. As discussed in the introduction, other factors may alter the effective mesh size and thus may produce $100 \%$ retention lengths different from those predicted by the equations. These factors may be incorporated into an analysis of mesh selection along with these regressions in order to better estimate the $100 \%$ retention length.

Saville (1958) showed that flexibility of both the organisms and the meshes can result in loss of organisms above the size predicted from mesh width. He determined that for $0.222 \mathrm{~mm}$ mesh the mean $100 \%$ retention width for seven zooplankton groups was $0.334 \mathrm{~mm}$, or $50.5 \%$ greater than the mean mesh size. Approximating the $100 \%$ retention size from his Figure 2 provides a diameter of $1.6 \mathrm{~mm}$ for fish eggs as caught by $1.188 \mathrm{~mm}$ mesh. This is a $35 \%$ increase over the mean mesh size. Experiments with glass beads suggested that both organism flexibility and mesh flexibility were 
involved in increasing the $100 \%$ retention size. Saville performed his experiments with silk gauze rather than with the nylon gauze in common use today. Silk is more extensible than nylon, so the problem of mesh flexibility for nets in common use today may not be quite as large as Saville's experiments indicated.

Heron (1968) discussed the variability of mesh size within one piece of gauze and suggested that a large part of Saville's organism and mesh flexibility may be explained by mesh size variability. As an organism moves from mesh to mesh toward the cod end of the net, it is likely to encounter meshes of larger and smaller size than the mean mesh size. If the mean mesh size and its standard deviation are known, then the mean plus upper $95 \%$ confidence limit provides a good estimate of the effective mesh size for $100 \%$ retention. This adjusted mesh size is actually a slight underestimate of the $100 \%$ retention size becuase only $97.5 \%$ of the meshes are smaller than this size.

One might predict that the faster a net is towed, and thus the greater the pressure drop across the meshes, the more organisms near the effective mesh size should be squeezed through the meshes. Tranter et al. (1968), however, observed that as net speed increased, the number of small organisms caught increased. They suggested that a larger pressure drop might trap organisms in their initial encounters with the net, and thus they are not exposed to other meshes through which they might pass. Much more work is needed if we are to understand the effects of hauling speed on mesh selection.

The regressions presented here provide estimates of chaetognath lengths given their widths. Assuming that width of the organism is the operating 
factor, these equations can be used to determine the lengths of chaetognaths sampled well by plankton nets of various mesh sizes. If the mean and standard deviation of the mesh material is known, then the mean plus upper $95 \%$ confidence limit is probably the best estimate of the effective mesh size. Another 10 to $20 \%$ may be added to the mesh size estimate to account for organism and mesh flexibility. This adjusted mesh size may be used to calculate the mean length plus upper $95 \%$ confidence limit, and this value should provide a reasonable estimate of the length above which retention is approximately $100 \%$.

Ideally, mesh selection should be tested in the field to determine minimum length for $100 \%$ retention for several nets of different mesh size. This is probably best accomplished by attaching several nets of different mesh size to a multiple net frame (such as a Bongo net). Many tows would be needed to reduce the effects of patchiness and the filtration efficiency of each net (especially for the smaller mesh sizes) would have to monitored closely with flowmeters. The minimum $100 \%$ retention sizes could then be determined for each mesh size by comparing the abundances within appropriate size class intervals. Such an experiment would allow us to test the usefulness of the regression equations in the analysis of mesh selection for chaetognaths.

Although they are underestimates of total population abundance, the MOC 1 abundance estimates are probably representative of the spatial and temporal abundance patterns for most of these species. 
D. Filtration volume

It is necessary to accurately measure the volume of water filtered by a plankton net in order to determine the abundance of the plankton in the water. The flowmeter of the MOC 1 was mounted on the frame of the net rather than in the mouth of the net, so it measured the distance traveled by the net relative to the water rather than the amount of water flowing through the mouth of the net. The filtration volume for each net is calculated as:

$\mathrm{V}=\mathrm{c} \cdot \mathrm{k} \cdot \mathrm{F} \cdot \mathrm{A}$

where $V$ is the volume filtered, $c$ is the flowmeter count, $k$ is the flowmeter calibration constant, $F$ is the filtration efficiency, and $A$ is the mouth area of the net. In practice the mouth area of the MOC 1 was calculated as the width $(1 \mathrm{~m})$ times the height $(1.4 \mathrm{~m})$ times $\cos \theta$, where $\theta$ was the angle of the net from the vertical. The MOC 1 was usually towed at an angle to the vertical of $45^{\circ}$, so the effective mouth area was $1 \mathrm{~m}^{2}$. The filtration efficiency of the net is defined as the proportion of water encountered at the net mouth which is actually filtered through the meshes of the net. The filtration efficiency of a net may be reduced during the course of a tow by clogging of the meshes with particulate matter in the water. The flowmeter counts and net angle were monitored throughout MOCNESS tows, so if we know the filtration efficiency we can calculate the volume of water filtered by each net.

Because there was no flowmeter within the mouth of the net, the 
filtration efficiency could not be measured directly. Tranter (1967) and Tranter and Smith (1968) provided several equations useful for the determination of the initial filtration efficiency of a net given several characteristics of the gauze and towing procedure. The filtration efficiency ( $F$ ) is calculated by the empirical relation:

$F=1 /(1+0.01 \cdot K)$,

(2) Tranter (12)

where $K$ is the resistance coefficient of the gauze. $K$ is a function of the porosity of the gauze (B) and its Reynolds' number (Re):

$\mathrm{K}=\left((1-\mathrm{B}) / \mathrm{B}^{2}\right) \cdot 6 \cdot \mathrm{Re}^{1 / 3}$.

(3) Tranter (6)

Porosity of the gauze is a measure of the fraction of the area of the gauze through which fluid can pass and is given by the equation:

$B=m^{2} /(m+d)^{2}$,

where $m$ is the mesh width and $d$ is the strand width (= diameter) of the gauze threads. The Reynolds' number is determined from the following equation:

$\operatorname{Re}=((A / a) \cdot U \cdot d) /\left(B \cdot v \cdot F_{b}\right)$

where $A$ is the mouth area of the net, $a$ is the mesh area of the net, $U$ is the towing velocity, $B$ and $d$ are as before, $v$ is the kinematic viscosity 
of water $\left(\sim 0.01 \mathrm{~cm}^{2} / \mathrm{s}\right)$, and $F_{b}$ is the filtration efficiency of the net frame without the net $\left(F_{b}\right.$ is assumed to be 1.0 here). The values of the variables necessary to calculate the initial filtration efficiency of the $\mathrm{MOCl}$ are provided in Table 3.10. These calculations yield an initial filtration efficiency of $0.95(95 \%)$ for the MOC 1 nets.

Clogging can reduce the filtration efficiency of the net during a tow. The quantitative effects of clogging are primarily dependent upon the size structure and species composition of the plankton being sampled and aspects of the net design such as shape and mesh size. Smith et al. (1968) presented an empirical relation for nets with $0.333 \mathrm{~mm}$ mesh by which the volume of water filtered before a reduction in filtration efficiency to 0.85 can be estimated as:

$\log _{10} R=0.37 \cdot \log _{10}(\mathrm{~V} / \mathrm{A})-0.49$ (6) Smith et al. (6)

where $R$ is the open area ratio of the net, $V$ is the volume filtered when filtration efficiency reaches 0.85 , and $A$ is the mouth area of the net. The open area ratio of the net is given by:

$R=a \cdot B / A$,

where $a, B$ and $A$ are the mesh area, porosity and mouth area of the net, respectively. Equation (6) was based upon observations of filtration efficiency of nets in 'blue water' off Catalina Island, California.

The MOC 1 nets were designed with an open area ratio of 5.3 (Wiebe et al., 1976) so that from equation (6) we predict that $1875 \mathrm{~m}^{3}$ of water 
can be filtered before a reduction of filtration efficiency to $85 \%$. MOC 1 nets usually filtered between 500 and $1000 \mathrm{~m}^{3}$ of oceanic water. The nets used by Smith et al. (1968) appear to have had the same initial filtration efficiency as the MOC 1 (0.95). Because each net should have filtered at greater than $85 \%$ efficiency during the tow and the volume of water filtered is usually about half the volume predicted from equation (6), it is probably safe to estimate that the filtration efficiency of each MOC 1 net was greater than 0.90 . That is, each net filtered greater than $90 \%$ of the water it encountered, but because an accurate estimate of the filtration efficiency was not available, filtration volumes were calculated on the basis of a filtration efficiency of 1.0 . Given the magnitude of some of the other sources of error for these samples, a possible $10 \%$ underestimate of the abundance of chaetognaths is minor.

Tranter (1967) and Tranter and Smith (1968) also provided equations from which the filtration pressure of the gauze can be calculated. The filtration pressure is defined as the drop in pressure from the inside surface of the gauze to its outside surface during the filtering process. Filtration pressure is probably an important factor affecting the condition of the catch and mesh selection. High filtration pressures may damage fragile organisms or push small organisms through the meshes. Filtration pressure $(P)$ is given by:

$P=\left(K \cdot P \cdot u_{a}^{2}\right) /(2 \cdot g)$,

(8) Tranter (1)

where $K$ is the gauze resistance coefficient, $p$ is the density of seawater $(1.026 \mathrm{~g} / \mathrm{cc}), \mathrm{u}_{\mathrm{a}}$ is the approach velocity of the water, and $\mathrm{g}$ is 
acceleration due to gravity. Tranter (1967) and Tranter and Smith (1968) failed to include $g$ in their equations, yet it is clear from Table 5 in Tranter and Smith (1968) that they used $g$ in their calculations. The inclusion of $\mathrm{g}$ in the equation converts filtration pressure from force per unit area $\left(\mathrm{g} / \mathrm{cm} \cdot \mathrm{s}^{2}\right)$ to weight per unit area $\left(\mathrm{g} / \mathrm{cm}^{2}\right)$. The approach velocity $\left(u_{a}\right)$ is the velocity of the fluid normal to the gauze and is given by:

$u_{a}=U \cdot \cos \theta$ or $u_{a}=U \cdot A / a$,

where $U, A$ and $a$ are the towing velocity, mouth area and mesh area, respectively, and $\theta$ is the side angle of the net. For a conical net, A/a is equal to $\cos \theta$. The average velocity through the meshes $\left(u_{m}\right)$ is calculated as :

$u_{\mathrm{m}}=u_{\mathrm{a}} / \mathrm{B}$

where $u_{a}$ is the approach velocity and $B$ is the net porosity. From these relations it can be seen that the Reynolds' number discussed earlier is, in effect:

$\operatorname{Re}=\left(u_{m} d\right) /\left(F_{b} v\right)$

A filtration pressure of $0.20 \mathrm{~g} / \mathrm{cm}^{2}$ was calculated for the MOC 1 nets. In comparison with commonly used nets from Table 5 of Tranter and Smith (1968), five nets had smaller filtration pressures and eleven nets 
had larger filtration pressures than the MOC 1. There should be less damage to the organisms and fewer organisms should be extruded through the meshes for the MOC 1 than for many zooplankton nets in common use today. 
Table 3.10. Filtration characteristics of the MOC 1 nets. 


\begin{tabular}{|c|c|c|}
\hline Variable & Designation & Value for $\mathrm{MOCl}$ nets \\
\hline Mouth area & $A$ & $1.0 \mathrm{~m}^{2}$ \\
\hline Mesh area & $\mathbf{a}$ & $11.8 \mathrm{~m}^{2}$ \\
\hline Mesh size & $\mathbf{m}$ & $0.333 \mathrm{~mm}$ \\
\hline Strand width & $\mathrm{d}$ & $0.16 \mathrm{~mm}$ \\
\hline Side angle of net & $\theta$ & $85^{\circ}$ \\
\hline Porosity & B & 0.45 \\
\hline Open area ratio & $\mathbf{R}$ & 5.3 \\
\hline Frame filtration efficiency & $F_{b}$ & 1.0 (assumed) \\
\hline Towing velocity & $\mathrm{U}$ & $100 \mathrm{~cm} / \mathrm{s}$ \\
\hline Reynolds' number & $\operatorname{Re}$ & 30.1 \\
\hline Gauze resistance coefficient & $\mathbf{K}$ & 5.2 \\
\hline Initial filtration efficiency & $F$ & 0.95 \\
\hline Volume of water filtered at & $85 \%$ & \\
\hline filtration efficiency & $\mathrm{V}$ & $1875 \mathrm{~m}^{3}$ \\
\hline Approach velocity & $u_{a}$ & $8.5 \mathrm{~cm} / \mathrm{s}$ \\
\hline Mesh velocity & $u_{b}$ & $18.8 \mathrm{~cm} / \mathrm{s}$ \\
\hline Filtration pressure & $\mathrm{P}$ & $0.197 \mathrm{~g} / \mathrm{cm}^{2}$ \\
\hline
\end{tabular}




\section{Patchiness}

\section{Introduction}

Patchiness, or overdispersion (in the statistical sense), of plankton has been found on a wide variety of spatial and temporal scales (Haury et a1., 1978). Patchiness has assumed an important status in the study of plankton ecology for several reasons. Patchiness reduces the precision (increases the variability) of plankton abundance estimates from net tows and pump samples, patchiness of food organisms in both the horizontal and vertical dimensions is believed to be very important for the survival of many pelagic organisms, especially larval fish (Anraku, 1975; Lasker, 1975; Mullin and Brooks, 1976), and recent modeling efforts have shown that patchiness on small spatial scales can affect spatial distributions on larger scales (Steele, 1974). I will limit further discussion of patchiness to its role in producing sampling error.

A number of studies have been concerned with empirically describing patchiness and its effects on sampling error (Winsor and Walford, 1936; Winsor and Clarke, 1940; Barnes and Marshal1, 1951; Cassie, 1963; Wiebe, 1971, 1972; Wiebe and Holland, 1968). Patchiness is detected by comparison of sampling variability with the random expectation from the Poisson distribution. Fisher's index of dispersion:

$x_{(n-1)}^{2}=(n-1) s^{2} / \bar{x}$

is used most frequently to detect departures from the Poisson 
distribution, where $n$ is the number of samples, $s^{2}$ is the variance of the sample counts, and $\bar{x}$ is the mean sample count. If $s^{2} / \bar{x}$ is greater than one, the organisms are patchy or overdispersed, and the rarely observed inverse, $s^{2} / \bar{x}$ less than one, results from a uniform or underdispersed distribution.

A number of frequency distribution models have been fit to observed plankton distributions (Cassie, 1962; Barnes and Marshal1, 1951; Fasham et al, 1974), but there appears to be little or no reason to prefer one of these models over any of the others. Cassie (1968) suggested that the lognormal distribution "does as well as any". Use of the $\log _{10}(x+1)$ transformed data offers several advantages over the untransformed data. The $\log _{10}$ transformed data are often normally distributed, negative lower confidence limits are avoided and the correlation between the mean and variance is reduced or removed.

Cassie (1968), summarizing earlier work in the field, suggested that the equation:

$s^{2}=\bar{x}+c \bar{x}^{2}$

is a good predictor of the variance associated with samples of mean $\bar{X}$. He reported values of the coefficient $c$ ranging from 0.05 to 0.10 , with higher values not uncommon. It is clearly seen from this equation that the sample variance will be much greater than the Poisson variance for all but small values of the mean abundance.

In the present study, a limited number of samples were available to represent the zoogeographic and seasonal abundance patterns of chaetognaths in the western North Atlantic Ocean. Variability due to 
patchiness, if large enough, can make it difficult or impossible to detect spatial and temporal differences in abundance. Analysis of two horizontal transects in the Slope Water confirmed the patchy distribution of chaetognaths in this region. Variability of the $\log _{10}$ transformed chaetognath abundances from day/night replicate tows was smaller than that found for many other zooplankters. Geographic and some temporal abundance differences were detected for many of these species, even with the large variability in these data.

Methods

Samples were collected and chaetognath species enumerated as discussed in Chapter 2. Two horizontal tows taken at $350 \mathrm{~m}$ in the Slope Water were available for the analysis of coarse scale variability in chaetognath abundance. Six and eight samples were available from MOC 1-22 and MOC 1-43, respectively, and each sample represented a distance of $500 \mathrm{~m}$ and a volume of $500 \mathrm{~m}^{3}$ water filtered. Fisher's index of dispersion was used to test for randomness of each species' abundance in these samples. The standard deviation $(s)$ of the $\log _{10}(x+1)$ transformed species' counts were also calculated. From the standard deviation, both the $95 \%$ confidence limits for a single observation and the logarithmic coefficient of variation $\left(\mathrm{CV}_{\log }\right)$ were calculated. Confidence intervals were calculated as percentages of the mean from the following equations:

Upper $95 \%$ confidence limit $=10010^{t \cdot s}$ and 
Lower $95 \%$ confidence 1 imit $=100 / 10^{t \cdot s}$,

where $t$ is the Student's t-distribution value for a probability of 0.05 and $(n-1)$ degrees of freedom and $s$ is ţhe standard deviation of the $\log _{10}$ transformed abundances. The logarithmic coefficient of variation is not very appropriately named, but its value is given by the following equation:

$C V_{10 g}=\left(10^{S}-1\right)+100$

The $\mathrm{CV}_{10 g}$ should not be confused with and is not equal to the coefficient of variation of the untransformed data (Bagenal, 1955). It is very useful, however, as a means of comparing the variablity between species.

I also used the data from the horizontal tows to calculate the value of the coefficient c from equation (2). The equation can be linearized as follows:

$s^{2} / \bar{x}=1+c \bar{x}$,

where $s^{2}$ and $\bar{x}$ are the variance and mean of the untransformed data, respectively. I calculated values of $s^{2} / \bar{x}$ for all the species in these two tows and used the values of $s^{2} / \bar{x}$ and $\bar{x}$ in a geometric mean regression (Ricker, 1973). The slope of this regression is $c$, and the $y$-intercept should be close to its expected value of one from equation (5). The results of this analysis will be used primarily in a comparison 
of subsampling and small scale patchiness variability to be discussed in the section on subsampling.

Sixteen day/night replicates tows were available for further analysis of the variability due to small scale patchiness. Members of a day/night pair were usually taken within several kilometers of one another. Fewer than sixteen pairs were actually available for any species because samples were missing from some of the tows (see Table 2.1). If the missing samples were from the depth range normally occupied by a species, that day/night pair was eliminated from the analysis for that species. The variance for each species in each day/night pair was calculated for $\log _{10}(x+1)$ transforms of abundance. For each species, the sum of the variances for all day/night pairs was divided by the number of day/night pairs for that species to estimate its average variance. The standard deviation was calculated from the average variance and was then used to calculate $95 \%$ confidence limits and the logarithmic coefficient of variation.

Usually, between two and four tows were taken within each of two hydrographic regions (SW and NSS) on each of the six cruises. As an example, when four tows were taken, they usually represented two day/night replicate pairs, the members of each pair usually separated by several kilometers and the pairs separated by tens to hundreds of kilometers. Eight sets of tows were available for the analysis of the large scale patchiness, but fewer were available for any particular species for the reason discussed earlier. The variance of these within cruise, within hydrographic region sets was calculated for each species based upon a nested analysis of variance model. The within set variance was tested for equality with the day/night replicate variance using the F-test. The 
within set standard deviation was used to calculate $95 \%$ confidence limits and the logarithmic coefficient of variation for each of these species.

Several caveats must be issued before proceeding to an analysis of the results. The samples collected within cruises were not synoptic. The variability within hydrographic regions and cruises thus includes both spatial and temporal components. I have assumed here that the spatial components of the variability are much greater than the temporal components within cruises. Also, for the within replicate and within hydrographic region and cruise variability, I have assumed that the variability of any species was the same in both the slope Water and the Northern Sargasso Sea. Although there may be a difference in the spatial variability between these regions for any particular species, I did not observe any such trends in these data. Lastly, it may not be appropriate to combine data from different species in the calculation of the coefficient $c$ from equation (2), but there is not enough data to calculate c separately for each species.

\section{Results}

\section{Horizontal tow variability}

Nine of the twenty one chi-square values for species in the horizontal tows were significantly greater than expected from the random Poisson distribution (Table 3.11). Nine of the twelve nonsignificant chi-square values were for chaetognaths with mean abundances of less than 5.0. A lack 
Table 3.11. Patchiness of chaetognaths in two horizontal transects at $350 \mathrm{~m}$ in the Slope Water $(*=p<0.05, * *=p<0.01$, $* * *=p(0.001)$. 


\begin{tabular}{|c|c|c|c|c|c|c|}
\hline Species & MOC 非 & $\mathbf{N}$ & $\begin{array}{l}\operatorname{Mean}(\text { 非/ } \\
\left.500 \mathrm{~m}^{3}\right)\end{array}$ & Variance & $x^{2}$ & $\mathrm{CV}_{10 \mathrm{~g}}$ \\
\hline \multirow[t]{2}{*}{ E. hamata } & 22 & 6 & 126.3 & 304.7 & $12.7 *$ & $15 \cdot 3$ \\
\hline & 43 & 8 & 41.7 & 165.6 & $27.8 * * *$ & 44.0 \\
\hline \multirow[t]{2}{*}{ E. bathypelagica } & 22 & 6 & 0.17 & 0.17 & $5.0 \mathrm{NS}$ & - \\
\hline & 43 & 8 & 0.12 & 0.12 & $7.9 \mathrm{NS}$ & - \\
\hline \multirow[t]{2}{*}{ K. subtilis } & 22 & 6 & 3.17 & 4.56 & $7.2 \mathrm{NS}$ & 99.9 \\
\hline & 43 & 8 & 8.60 & 42.1 & $34.3 * \star *$ & 82.5 \\
\hline K. pacifica & 43 & 8 & 0.37 & 1.09 & $20.7 * \star$ & - \\
\hline \multirow[t]{2}{*}{ S. decipiens } & 22 & 6 & 23.7 & 111.1 & $23.5 * * *$ & 60.0 \\
\hline & 43 & 8 & 30.2 & 306.8 & $71.0 * * *$ & 92.3 \\
\hline S. enflata & 43 & 8 & 1.57 & 7.36 & $32.8 * * *$ & - \\
\hline \multirow[t]{2}{*}{ S. 1yra } & 22 & 6 & 12.2 & 19.0 & $7.8 \mathrm{NS}$ & 36.8 \\
\hline & 43 & 8 & 2.30 & 0.48 & $1.5 \mathrm{NS}$ & 26.5 \\
\hline S. macrocephala & 43 & 8 & 0.25 & 0.21 & $5.9 \mathrm{NS}$ & - \\
\hline \multirow[t]{2}{*}{ S. maxima } & 22 & 6 & 12.2 & 22.2 & $9.1 \mathrm{NS}$ & 61.0 \\
\hline & 43 & 8 & 18.3 & 37.7 & $14.4 *$ & 34.9 \\
\hline S. minima & 43 & 8 & 0.49 & 0.53 & 7.6 NS & - \\
\hline \multirow[t]{2}{*}{ S. planctonis } & 22 & 6 & 0.33 & 0.27 & $4.0 \mathrm{NS}$ & - \\
\hline & 43 & 8 & 0.99 & 6.20 & $0.2 \mathrm{NS}$ & 49.8 \\
\hline S. serratodentata & 43 & 8 & 0.25 & 0.21 & $5.9 \mathrm{iNS}$ & - \\
\hline \multirow[t]{2}{*}{ S. tasmanica } & 22 & 6 & 1.00 & 1.20 & $6.0 \mathrm{NS}$ & - \\
\hline & 43 & 8 & 6.17 & 17.8 & $20.2 * *$ & 93.2 \\
\hline
\end{tabular}


of significance for such small values of the mean was expected (Cassie, 1968). Actual variations in abundance along the horizontal transects are shown in Figure 3.2, and these variations are seen to occur on scales larger than the $500 \mathrm{~m}$ sample lengths (e.g. E. hamata, MOC 1-43). Also presented in Table 3.11 are the $95 \%$ confidence limits for a single observation and the logarithmic coefficients of variation for chaetognaths with mean abundance greater than 2.0 . Because the organism counts are discrete rather than continuous, the variability in the abundance of these rare $\left(<2.0\right.$ individuals $\left./ 500 \mathrm{~m}^{3}\right)$ species can be quite large for the $\log _{10}$ transformed data. The mean logarithmic coefficient of variation for twelve species shown was $58.0 \%$, with a range from 15.3 to $99.9 \%$. The geometric mean regression of $s^{2} / \bar{x}$ and $\bar{x}$ resulted in the following equation:

$s^{2} / \bar{x}=1.31+0.0807 \bar{x}$ or

$s^{2}=1.31 \bar{x}+0.0807 \bar{x}^{2}$

The correlation coefficient was 0.254 , a value not significantly different from zero. An examination of the data presented in Figure 3.3 shows that one data point (E. hamata, $\overline{\mathrm{x}}=126.3, \mathrm{~s}^{2} / \overline{\mathrm{x}}=2.412$ ) was isolated from the rest of the points. After the exclusion of this point from the data, the geometric mean regression equation was:

$s^{2} / \bar{x}=0.746+0.200 \bar{x}$ or 
Figure 3.2. Small scale spatial distributions of chaetognaths at 350 $m$ in the Slope Water. Asterisks denote lost samples. 

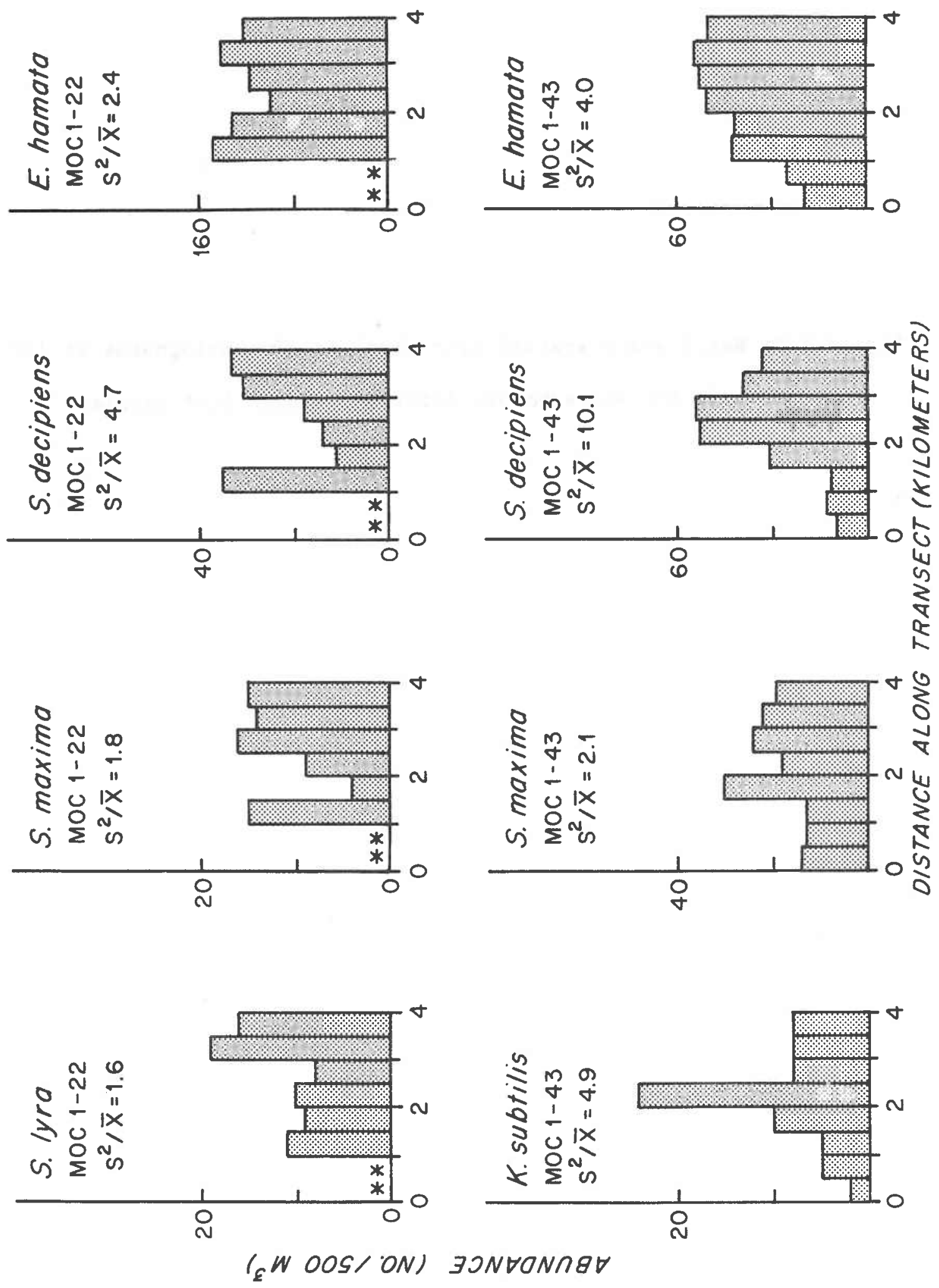
Figure 3.3. Relationship of $\mathrm{s}^{2} / \overline{\mathrm{x}}$ and $\bar{x}$ for the chaetognaths from the horizontal tows in the SW. Line 2 is the geometric mean regression for all the data and Line 1 is the regression excluding the point, $\bar{x}=126, \mathrm{~s}^{2} / \bar{x}=2.4$. 


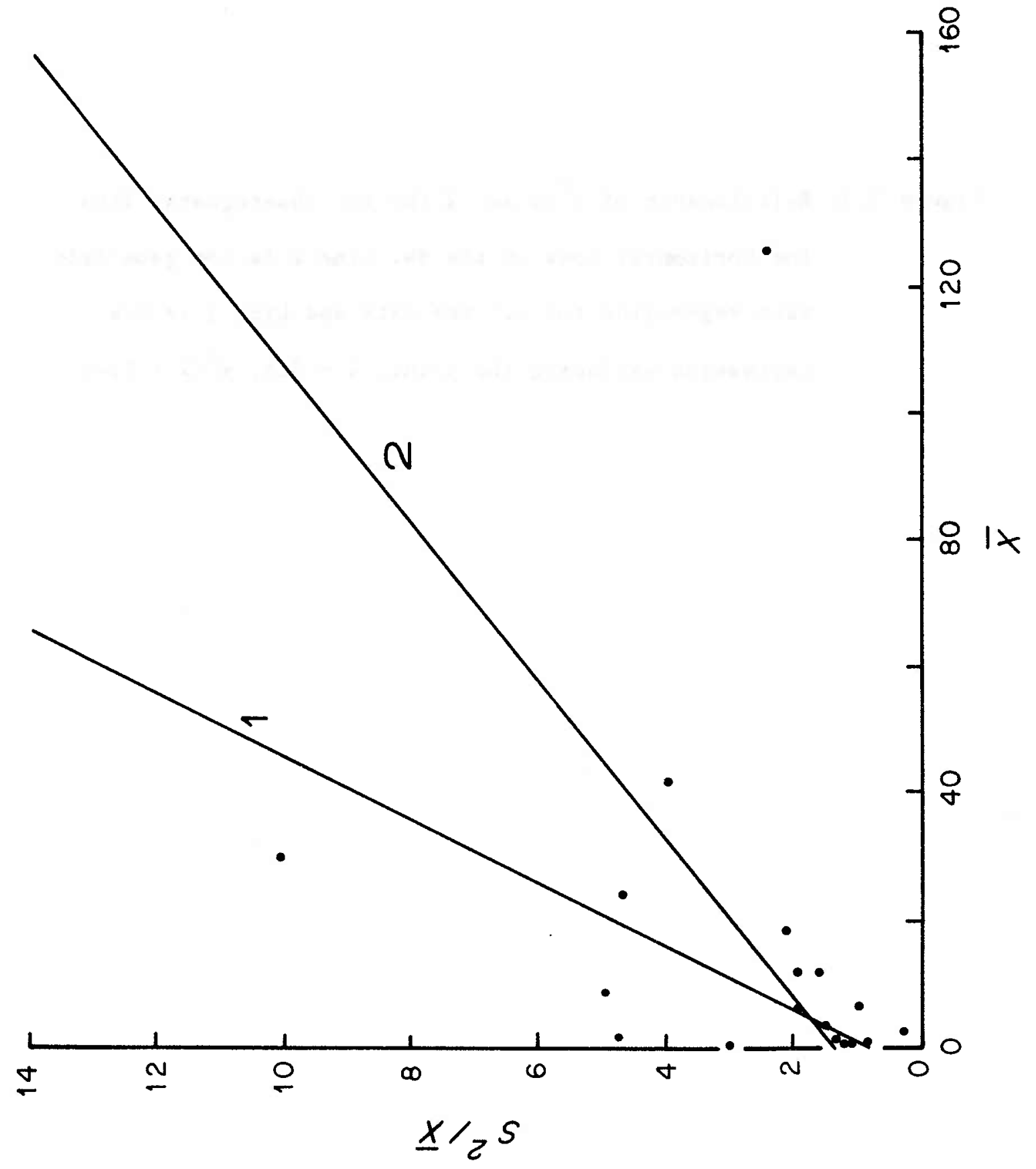


$s^{2}=0.746 \bar{x}+0.200 \bar{x}^{2}$,

with a correlation coefficient of 0.635 ( $p$ 0.01). In both equations (7) and $(y)$, the $y$-intercept was close to its expected value of one. The true value of the coefficient $c$ for these data probably lies somewhere between the values of 0.0807 and 0.200 . Cassie (1968) quoted a range of values from 0.05 to 0.10 as typical for plankton.

Paired tow variability

Paired day/night tows separated by a few kilometers yielded variabilities for species counts of the same order as found for the horizontal tows (Table 3.12). The average logarithmic coefficient of variation for seventeen species was $59.3 \%$, with a range from 17.5 to 176 $\%$. It should be noted that the variability within replicate tows is due both to patchiness and subsampling. Subsampling variability may be an important component of the observed variability for some of these species (see Discussion).

The replicate tow variability appears to be a function of the depth of occurrence of these species. The mean logarithmic coefficient of variation value was $24.0 \%$ for bathypelagic species, $44.5 \%$ for mesopelagic species, and $88.7 \%$ for epipelagic species, and these values were significantly different from one another (Mann-Whitney u-test, $p<0.01$ ). One might expect such a pattern because spatial and temporal fluctuations in the oceanic environment decrease as depth increases. This pattern may also be attributed to subsampling variability. Because chaetognath abundance was 
Table 3.12. Variability of chaetognath abundance estimates within day/night replicates and within hydrographic region and cruise sets. 
Species

Within replicate variability

$95 \%$ confidence limits
$(\%$ of mean $)$

Bathypelagic species

E. bathypelagica

$64-156$

9

21.6

E. fowleri

$58-174$

13

29.4

E. hamata

$67-150$

11

20.4

S. macrocephala

$56-180$

13

31.3

S. maxima

$70-143$

11

17.5

Mesopelagic species

K. subtilis

S. decipiens

S. lyra

S. planctonis

Epipelagic species

$\begin{array}{lll}49-206 & 13 & 40.0 \\ 40-253 & 15 & 54.6 \\ 48-210 & 14 & 41.3 \\ 46-215 & 12 & 42.1\end{array}$

$11-912$

12

176

$23-441$

14

99.8

$21-488$

12

107

$34-295$

15

66.2

$35-283$

15

63.0

$39-256$

13

54.4

31 - 319

15

72.4

$30-337$

9

71.1 
Table 3.12. (cont'd).

Within set variability
$95 \%$ confidence
$(\%$ of mean)
$\mathrm{CV}_{1 \mathrm{og}}$
$\mathrm{H}_{\mathrm{o}}: \mathrm{s}_{\mathrm{r}}^{2}=\mathrm{s}_{\mathrm{s}}^{2}$

$\begin{array}{llllc}\text { E.b. } & 51-195 & 5 & 29.7 & \text { NS } \\ \text { E.f. } & 42-238 & 7 & 44.4 & \text { NS } \\ \text { E.h. } & 34-292 & 5 & 51.7 & \mathrm{p}<0.01 \\ \text { S.m. } & 23-441 & 8 & 90.3 & \mathrm{p}<0.001 \\ \text { S.m. } & 16-607 & 7 & 114 & \mathrm{p}<0.001\end{array}$

$\begin{array}{lllll}\text { K.s. } & 36-274 & 7 & 53.2 & \text { ISS } \\ \text { S.d. } & 35-286 & 7 & 55.9 & \text { NS } \\ \text { S.1. } & 36-278 & 7 & 54.1 & \text { NS } \\ \text { S.P. } & 32-313 & 8 & 63.9 & \text { NS }\end{array}$

$\begin{array}{lrrrr}\text { K.p. } & 11-912 & 7 & 176 & \text { NS } \\ \text { P.d. } & 19-514 & 8 & 103 & \text { NS } \\ \text { S.b. } & 16-604 & 7 & 114 & \text { NS } \\ \text { S.e. } & 8-1240 & 8 & 197 & \text { p }<0.001 \\ \text { S.h. } & 25-404 & 8 & 83.2 & \text { NS } \\ \text { S.m. } & 9-1144 & 7 & 180 & \text { p }<0.001 \\ \text { S.s. } & 31-319 & 8 & 72.4 & \text { NS } \\ \text { S.t. } & 10-994 & 5 & 144 & \text { P }<0.05\end{array}$


greatest in the surface waters, the shallower living species were more subject to subsampling than were the deeper living species. How might we test the hypothesis that the increased variability of the surface species was due to subsampling variability? The value of $s_{s} / s_{p}$ from Table 3.15 of the subsampling section is a measure of the relative contributions of subsampling and patchiness to the species' abundance variability. The larger the value of $s_{s} / s_{p}$, the greater the contribution of subsampling to the total variability observed. A Spearman rank correlation coefficient should reveal a positive relationship between $s_{s} / s_{p}$ and the $\mathrm{CV}_{10 g}$ from Table 3.12 , if subsampling was a major component of the variability for these species. In order to remove the effect of depth of occurrence, I calculated the Spearman rank correlation coefficient for only the epipelagic species, and its value was $0.857(p<0.05)$. Thus we can assume that a large part of the differences in variability for these species was due to subsampling. The variability due to patchiness may still be a function of the depth of occurrence, but the differences are not as great as indicated in Table 3.12.

Within hydrographic region and cruise variability

Variability in species abundance within hydrographic regions and cruises was in general greater than that for day/night pairs (Table 3.12). Six of the seventeen species showed significantly greater variability on the larger spatial scale, and all but two species showed some increase in variability on the larger scale. The mean $\mathrm{CV}_{10 g}$ for the within hydrographic region and cruise variability was $95.7 \%$, with a range from 
29.7 to $197 \%$.

\section{Discussion}

The results of this analysis show that chaetognaths in the western North Atlantic Ocean are patchily distributed on several spatial scales. The spatial scales for which patchiness was detected in this study include the fine scale ( $10^{\prime}$ 's to 100 's of meters; horizontal tows), coarse scale ( 1 to $10^{\prime} \mathrm{s}$ of kilometers; replicate day/night pairs), meso-scale (100's of kilometers; within hydrographic region and cruise tows and Gulf Stream rings), and macro-scale (1000's of kilometers; zoogeographic patterns). The small (fine and coarse) scale patchiness often makes it difficult to detect spatial or temporal differences in chaetognath abundance on larger scales.

The degree of variability observed for these chaetognaths is smaller than the variability observed for many other zooplankters. Table 3 of Wiebe and Holland (1968) provided $95 \%$ confidence limits for zooplankters from eleven previous studies of small scale patchiness of zooplankton. I converted these confidence limits to logarithmic coefficients of variation, assuming $t_{.05}$ was 2.0 . The $\mathrm{CV}_{10 \mathrm{~g}}$ for these studies ranged from 30.0 to $297 \%$, with a mean of $81.4 \%$. The mean $\mathrm{CV}_{10 g}$ for twenty categories of zooplankton sampled at night and reported by Miller (1969) was $102 \%$, with a range from 31.2 to $454 \%$. The mean for twenty categories of zooplankton sampled during the day was $166 \%$, with a range from 50.6 to $402 \%$. Assuming a t.05 value of $2.0,95 \%$ confidence limits for nine 
categories of zooplankton reported by wiebe et al. (1973) yielded a range from 55.2 to $156 \%$ and a mean of $85.2 \%$. The $95 \%$ confidence limits for a single observation and the $\mathrm{CV}_{10 g}$ values for several chaetognath species from published reports are given in Table 3.13. Sameoto (1978) published $95 \%$ confidence limits for Sagitta spp. (probably S. elegans) off Nova Scotia, but he obviously did not calculate his confidence limits as I have done, nor can I determine how he calculated them based on the reference (Bagenal, 1955) he cited. It is clear that the variability in these published reports is much larger than that observed in this study.

I can offer several hypotheses to explain the smaller variability found in this study. Most of the previous studies were done in neritic waters which are generally more variable than oceanic waters. Most of these studies also include some subsampling variability in addition to that due to small scale patchiness. Probably the most important feature of the studies from Table 3.13 is that less than $200 \mathrm{~m}^{3}$ of water was filtered for each sample and the longest tows were $600 \mathrm{~m}$ in length. Wiebe (1971, 1972), in a theoretical study of patchiness and subsequent field test of the model, showed that variability in zooplankton abundance from repeated tows (measured as the coefficient of variation) could be reduced by increasing the volume of water filtered by the net. The variability was reduced most when the tow length was increased rather than increasing the mouth area of the net. Each MOC 1 net usually filtered between 500 and $1000 \mathrm{~m}^{3}$ along an oblique tow path of 500 to $1000 \mathrm{~m}$. All nets combined from a tow usually sampled from 4000 to $6000 \mathrm{~m}^{3}$ of water along an oblique tow path of 4000 to $6000 \mathrm{~m}$. Many of these chaetognaths occurred over several depth intervals sampled, so that typically, each MOC 1 tow 
Table 3.13. Small scale variability of chaetognath abundance from published reports. 


\begin{tabular}{|c|c|c|c|}
\hline Species & $\begin{array}{l}\text { confidence limits } \\
(\% \text { of mean })\end{array}$ & $\mathrm{CV}_{1 \circ \mathrm{g}}$ & Reference \\
\hline S. elegans, $75 \mathrm{~cm}$ net & $13-799$ & 151 & Winsor and Clarke, \\
\hline S. elegans, $30.5 \mathrm{~cm}$ net & $17-594$ & 120 & 1940; Table IV \\
\hline S. elegans, $12.7 \mathrm{~cm}$ net & $28-363$ & 76.9 & \\
\hline S. elegans, $75 \mathrm{~cm}$ net & $12-850$ & 158 & Winsor and Clarke, \\
\hline S. elegans, $12.7 \mathrm{~cm}$ net & $20-492$ & 102 & 1940; Table VIII \\
\hline S. elegans, $12.7 \mathrm{~cm}$ net & $10-995$ & 180 & \\
\hline S. enflata & $29-341$ & 80.6 & Miller, 1968; Night \\
\hline S. pseudoserratodentata & $41-243$ & 53.5 & \\
\hline Inmature Sagitta & $17-590$ & 137 & \\
\hline S. bieri & $27-368$ & 86.4 & Miller, 1968; Day \\
\hline S. euneritica & $13-771$ & 165 & \\
\hline Immature Sagitta & $33-306$ & 70.7 & \\
\hline S. elegans, eggs & $30-346$ & 86.0 & Wiebe et al., 1973 \\
\hline S. elegans, juveniles & $40-251$ & 58.4 & \\
\hline Sagitta spp., Roseway & $5-721$ & ? & Sameoto, 1978 \\
\hline Sagitta spp., Roseway & $9-429$ & ? & \\
\hline Sagitta spp., Random & $12-394$ & $?$ & \\
\hline Sagitta spp., Shelf & $0.3-735$ & $?$ & \\
\hline S. elegans & $71-140$ & 18.3 & King, 1979 \\
\hline
\end{tabular}


sampled an oblique path from about 1000 to $3000 \mathrm{~m}$ in length over the habitat occupied by each species. This increase in tow length most likely resulted in the reduced variability in chaetognath abundance on the small scale in this study relative to previous studies.

The value of the coefficient $c$ from equation (2) may also be thought of as an index of patchiness. The larger the value of $c$, the greater the variability from repeated tows. Winsor and Walford (1936) first proposed this equation to account for variability introduced in the volume of water filtered. It has been found to provide a reasonable empirical prediction of the variance expected from a given mean abundance, but it should not be thought of as a theoretical model. The coefficient c can take on a rather wide range of values, from the value of 0.05 reported by Winsor and Walford (1936) to the value of 0.40 approximated from Miller's (1969) data. The value of $c$ probably depends upon a number of variables such as the species counted, the sampling gear used, and the time and place of sampling. It is interesting to note that equation (2) predicts that the coefficient of variation should decrease with increasing abundance to an asymptotic value of $c^{1 / 2}$. Thus an increase in the volume of water filtered (thus increasing the abundance of zooplankton in the sample) results in a decrease in the coefficient of variation, just as Wiebe's $(1971,1972)$ studies showed. Wiebe found that increased tow length reduced the coefficient of variation much more than an equivalent increase in volume filtered by increasing the mouth area, however. The coefficient $c$ may be a function of the tow length as well as mean abundance. The coefficient of variation should decrease as tow length increases, but at larger towing distances the coefficient of variation will again increase 
due to patchiness on larger scales.

The variability between widely spaced stations within a hydrographic region was found to be greater than the small scale variability. Because we have too few samples, it is not clear how representative our abundance estimates were of the larger hydrographic regions. An analysis of variance on the $\log _{10}(x+1)$ transformed abundances for each of the species in this study showed that even with the large scale variability it was possible to detect the large scale zoogeographic and some temporal differences in abundance. Fifteen of seventeen species showed significant $(p<0.05)$ differences in abundance between the Slope Water and the Northern Sargasso Sea. The two nonsignificant species had probabilities of 0.052 and 0.065 , suggesting that there were large scale spatial trends for all these species. In addition, significant $(p<0.05)$ differences between cruises (assumed to represent seasonal differences) were detectable for eleven of the seventeen species. The small scale variability and that within hydrographic regions and cruises were not large enough to obscure all the spatial and temporal abundance patterns of these species.

These estimates of variability may be useful in designing future sampling programs. They may be especially useful for designing field tests of model predictions. As an example, suppose we have a model which predicts that the abundance of $\underline{S}$. decipiens in the Northern Sargasso Sea in summer should be twice its winter value. Given the variability in abundance estimates from this study, how many samples are necessary to detect a two-fold difference in abundance significant at the 0.05 level? Assuming that we collect equal numbers of samples at both times, we can calculate the necessary number of samples from the t-test formula: 
$t=\left(x_{1}-x_{2}\right) /\left(2 s^{2} / N\right)^{1 / 2}$

where $x_{1}$ and $x_{2}$ are the $\log _{10}(x+1)$ transforms of $S_{\text {. decipiens }}$ abundance at the two time points, $s^{2}$ is the variance of $\underline{s}$ decipiens abundance from Table 3.12 (calculated from the $\mathrm{CV}_{\log }$ ), and $\mathrm{N}$ is the number of samples needed for each time point. For a two-fold difference in abundance, $x_{1}-x_{2}=\log _{10}(2)=0.301$, and $s^{2}$ calculated from the $\mathrm{CV}_{\text {log }}$ from Table 2 (the within hydrographic region and cruise variance) is 0.0372 . Several iterations of equation (11) may be necessary to determine the appropriate number of samples since both the left and right sides of the equation are functions of $\mathrm{N}$. For this example, six samples at each time produce a $t$ value of 2.70 , and $t .05(5)$ is 2.57 . 
E. Subsampling

Introduction

Zooplankton samples often contain thousands to hundreds of thousands of individual organisms. Any one species may be represented by up to several thousand individuals. Sorting and identifying the organisms in these samples is a slow, laborious and therefore expensive process. It is often necessary to subsample in order to reduce the number of individuals to a manageable level for sorting and identification. Subsampling, however, introduces another component of variability in the abundance estimates. It is necessary to understand the magnitude of subsampling variability so that the desired level of variability may be balanced against the time and effort needed to sort and identify zooplankton.

The following theoretical framework can be applied to any subsampler, but I will limit further discussion to the subsampling properties of the Folsom plankton sample splitter (McEwen et al., 1954). The Folsom splitter divides a sample suspended in liquid into two approximately equal parts. Each of the subsamples may be further split (up to m times) so that final aliquots of $1 / 2,1 / 4,1 / 8, \ldots, 1 / 2^{\text {m }}$ of the original sample volume may be obtained. The value $1 / 2^{\mathrm{m}}$ also represents the probability that any particular individual will be present in the final aliquot. If $N$ is the number of individuals in the original sample, $p$ is the probability of an individual's presence in the final aliquot, and $q$ is the probability of not being in the final aliquot $(1-p)$, the probability of obtaining any particular outcome from a subsampling experiment is given by the binomial 
expansion, $(p+q)^{N}$. The expected number of organisms in the final aliquot is:

$\mathrm{u}=\mathrm{N} \cdot \mathrm{p}$ or $\mathrm{u}=\mathrm{N} / 2^{\mathrm{m}}$,

and the expected variance about the aliquot count for the binomial distribution is:

$s_{a}^{2}=N \cdot p \cdot q$ or $\quad s_{a}^{2}=\left(N / 2^{m}\right)\left(1-1 / 2^{m}\right)$.

The expected variance about the total sample count $N$ is:

$s_{N}^{2}=N\left(2^{m}-1\right)$

The expected standard deviation about $\mathrm{N}$ for increasing values of $\mathrm{N}$ are shown in Figure 3.4 for aliquots of $1 / 2,1 / 4,1 / 8,1 / 16$.

If the organisms are randomly distributed in the aliquots, then equation (2) gives the expected variance. If the probability of an individual's presence in the final aliquot is not independent of the presence of other individuals in the final aliquot, then the observed variance will be either greater or less than the expected variance, depending upon whether the organisms tend to clump together or are distributed uniformly during the subsampling process. Although I cannot determine a theoretical basis for expecting nonrandomness, Murphy's laws suggest that the aliquot counts should be nonrandom and the observed variance should be greater than expected. The appropriate test for nonrandomness is the chi-square test: 
$x_{(n-1)}^{2}=(n-1) s^{2} /(N \cdot p \cdot q)$

where $\mathrm{n}$ is the number of aliquots counted, $\mathrm{s}^{2}$ is the observed variance of the aliquot counts, and $\mathrm{N} \cdot \mathrm{p} \cdot \mathrm{q}$ is the expected variance from equation (2). A chi-square value greater than $\mathrm{n}-1$ (or $\mathrm{s}^{2}>\mathrm{N} \mathrm{p} q$ ) indicates a clumped distribution, while a value less than $n-1$ (or $s^{2}<N$ p $q$ ) indicates a uniform distribution in the aliquots. The significance of the chi-square value with $n-1$ degrees of freedom can be determined from the appropriate statistical tables.

The Poisson distribution has occassionally been applied to subsampling variations (Venrick, 1971), and tests for randomess of aliquot counts have sometimes been based upon this distribution (Miller, 1969). The Poisson distribution is an approximation to the binomial when the probability of a discrete event occurring is very low. If the event is the occurrence of an individual in the final aliquot, then the Poisson is an appropiate approximation when the aliquot size is small. The expected number in the aliquot is $\mathrm{N} \cdot \mathrm{P}$ as in the binomial distribution, but the variance of the Poisson is $N \cdot p$, as opposed to $N \cdot p \cdot q$ for the binomial. As the value of $p\left(=1 / 2^{m}\right)$ decreases, the value of $q$ approaches one. The soundness of Poisson approximation depends upon the closeness of $q$ to a value of one. Values of $p$ and $q$ for decreasing aliquot size are as follows:

$\begin{array}{ccccccccc}\text { \# splits } & 1 & 2 & 3 & 4 & 5 & 6 & 7 & 8 \\ \mathrm{p} & 0.500 & 0.250 & 0.125 & 0.063 & 0.031 & 0.016 & 0.008 & 0.004 \\ \mathrm{q} & 0.500 & 0.750 & 0.875 & 0.937 & 0.969 & 0.984 & 0.992 & 0.996\end{array}$


The Poisson distribution should be a very close approximation to the binomial when the aliquot size is less than $1 / 32\left(1 / 2^{5}\right)$ of the original sample volume.

The test most often applied to detect randomness of aliquot counts has been the chi-square goodness of fit test:

$x_{(n-1)}^{2}=\sum\left(c_{i}-\bar{c}\right)^{2} / \bar{c}$

where $\mathrm{n}$ is the number of aliquots counted, $\mathrm{C}_{i}$ is the observed count from the $i^{\text {th }}$ aliquot, and $\bar{C}$ is the mean aliquot count. The appropriate chi-square goodness of fit test for a single aliquot where the mean count is known is, however:

$x_{(1)}^{2}=\left(C_{i}-\bar{C}\right)^{2} / \bar{C}+\left(\left(N-C_{i}\right)-(N-\bar{C})\right)^{2} /(N-\bar{C})$,

where the second term on the right side is the deviation from the expected number not in the final aliquot. For a series of aliquots from the same sample, the chi-square goodness of fit test is now:

$x_{(n-1)}^{2}=\Sigma\left(C_{i}-\bar{C}\right)^{2} / \bar{C}+\Sigma\left(\left(N-C_{i}\right)-(N-\bar{C})\right)^{2} /(N-\bar{C})$,

where $\mathrm{n}$ is the number of aliquots counted and $\mathrm{N}$ is the number of individuals in the original sample (estimated by $\overline{\mathrm{C}} \cdot 2^{\mathrm{m}}$ ). The chi-square goodness of fit statistic from equation (7) can be shown to be equivalent to the chi-square statistic from equation (4). The chi-square goodness of 
fit statistic from equation (5) is an appropriate test for randomness based upon the Poisson distribution, and its use as a test for the randomness of aliquot counts should be limited to small aliquots (less than $1 / 32$ of the original sample).

McEwen et al. (1954) concluded that the errors associated with the use of the Folsom splitter are random. Their chi-squared test for randomness was based on the average variance of the aliquot counts instead of the expected binomial variance. Miller (1969) calculated the chi-square goodness of fit test from equation (5) for the data presented by McEwen et a1. and he concluded that their aliquot counts showed significant nonrandomness. Since the appropriate chi-square statistic (from equation (4) or (7)) is larger in value than that from equation (5), a reanaly sis of their data using equation (4) would also indicate nonrandomess. McEwen et al. presented an equation for estimating the variance about the total sample count $\mathrm{N}$ as follows:

$$
s^{2}=0.028 \mathrm{~N}^{2} \mathrm{~F}_{\mathrm{v}}(\mathrm{m}) / \mathrm{V}^{1 / 2}+\mathrm{N} \cdot \mathrm{F}_{\mathrm{n}}(\mathrm{m})
$$

where $V$ is the volume of the original sample, and $F_{v}(m)$ and $E_{n}(m)$ are functions dependent upon the number of sample splits $m$. Values for $F_{v}(m)$ are obtained from the relationship $F_{v}(m+1)=F_{v}(m)+2^{(m+1) / 2}$ $\left(F_{v}(0)=0\right)$. Values of $F_{n}(m)$ are obtained from the equation $F_{n}(m)=$ $2^{m}-1$. The second term in equation ( 8 ) is thus equivalent to the binomial variance from equation ( 3 ). McEwen et al. attribute the second term to the random distribution of organisms suspended in liquid, while the first term is an empirical formulation of the variability of the 
volume of liquid in the final aliquot. Their total variance is therefore greater than that predicted from the binomial variance. The standard deviations associated with increasing values of $\mathrm{N}$ for aliquots of $1 / 2$, $1 / 4,1 / 8,1 / 16$ based upon equation (8) are shown in Figure 3.4 . Miller (1969) concluded from his analysis of the Folsom splitter results that the errors were nonrandom. Again, he used the chi-square statistic based on equation (5), and the use of the appropriate statistic from equation (4) confirms the nonrandomness of his data. Miller estimated the variance of aliquot counts by the following equation

$s^{2}=1.84 \mathrm{~N}\left(2^{\mathrm{m}}-1\right)$,

which was unfortunately based upon the erroneous chi-square statistic from equation (5). Miller's equation is included in Figure 3.4 for comparison with the prediction from the binomial distribution and McEwen et al.

I have performed experiments on the subsampling error of the Folsom splitter and I have reanalyzed data presented by Miller and by Longhurst and Siebert (1967). My data and Miller's support the hypothesis that subsampling errors are nonrandom and the variance is larger than expected. The observed variance can be estimated by multiplying the binomial variance by the ratio of the observed variance to the binomial variance as Miller did, but using the appropriate chi-square value. The relationship between the observed variance and the number of individuals in the original sample can be used to determine the aliquot size necessary to achieve a specified level of variability. A comparison of the variability due to subsampling and that due to small scale patchiness suggests that 
Figure 3.4. Relationship between the number of organisms in the total sample and the expected standard deviation of the total sample count for aliquots of $1 / 2,1 / 4,1 / 8$, and $1 / 16$ 


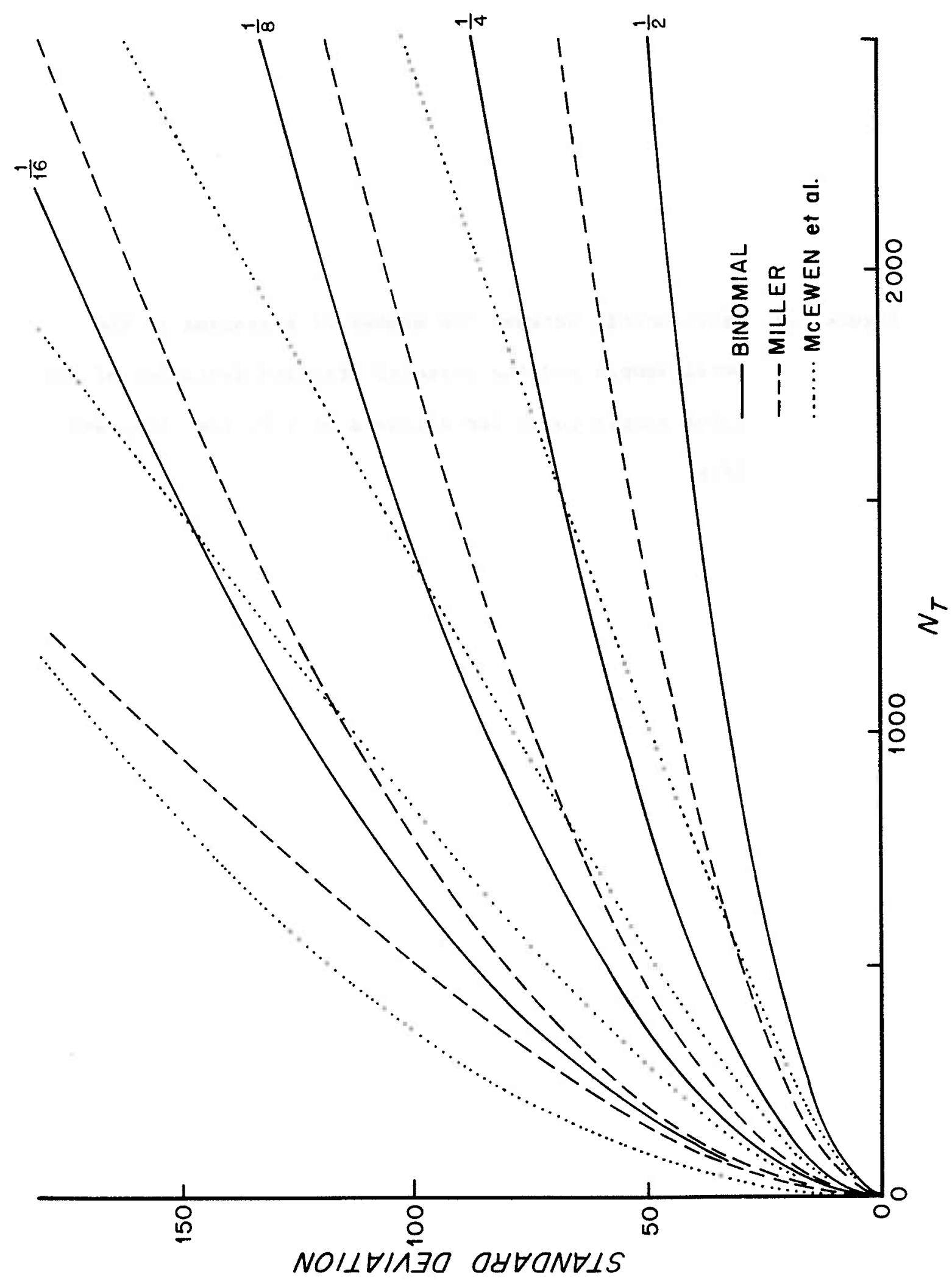


subsampling variability was a very important source of variability for some of the species in this study.

Methods

Data from three sources were used to test the randomness of the aliquot counts of chaetognaths from the Folsom plankton sample splitter. I obtained $1 / 2$ aliquots from 21 samples of mixed chaetognath species. The mixed species were suspended in approximately one liter of water, and atter pouring the sample into the splitter, the sample was stirred until it appeared we 11 mixed. For twenty of the samples, total chaetognaths from both of the resulting aliquots were counted and for the remaining sample the numbers of each species in both aliquots were counted. The variance of the aliquot counts was computed for each of the twenty total chaetognath samples and for ten species in the remaining sample.

In addition to the $1 / 2$ aliquots, $1 / 4$ aliquots were obtained from seven mixed chaetognath samples. For six of the samples, each species was counted in the $1 / 4$ aliquot and the remaining $3 / 4$ aliquot and for the remaining sample only total chaetognath number was counted in both the $1 / 4$ and $3 / 4$ aliquots.

Miller (1969) split ten mixed plankton samples twice to obtain four $1 / 4$ aliquots for each sample, and he counted two chaetognath species and several crustacean species in these aliquots. Miller presented all four $1 / 4$ aliquot counts for each sample, so it is possible to compute three variances for $1 / 2$ aliquots for each sample. The first split produces two 
$1 / 2$ aliquots for which a variance can be calculated. Splitting these two aliquots results in two more pairs of $1 / 2$ aliquots, and a variance can be calculated for each pair. In this manner, 60 variances ( 2 chaetognath species $\times 10$ samples $\times 3$ variances) for $1 / 2$ alquots were calculated from Miller's data. The variances of the $1 / 4$ aliquots were also calculated (2 species $\times 10$ samples $=20$ variances $)$.

Longhurst and Siebert (1967) split a mixed zooplankton sample to obtain $1 / 16$ aliquots. They did this six times for each of three different mixing techniques. They present the standard deviations of the aliquot counts for each of the three mixing methods, so three variance values for $1 / 16$ aliquots were available for a single chaetognath species.

In summary, for $1 / 2$ aliquots, 90 estimates of subsampling variance were obtained, for $1 / 4$ aliquots, 30 estimates were obtained, and for $1 / 16$ aliquots, three estimates were obtained. The chi-square value for each of these variances was calculated from equation (4) and significant departure from randomness was determined from Table $R$ in Rohlf and Sokal (1969).

The samples used in the above analyses were not the original plankton samples, but instead were made by mixing together several sorted zooplankton populations. Although the performance of the Folsom splitter may not be exacly the same for these mixed samples as with the original plankton samples, the results discussed here are probably reasonable approximations to those expected from the original samples. 
Results

The variance of the aliquot counts was generally greater than expected from the binomial distribution (Figure 3.5). Of the 90 variances for $1 / 2$ aliquots, fourteen were significantly greater than expected and two were significantly less than expected at the 0.05 level. The expected number of significant differences was $4.5(=90 \times 0.05)$ if the aliquot counts were random. The probability of obtaining sixteen or more significant differences when 4.5 are expected in 90 trials is less than 0.001 . Because chi-square values are approximately additive (Sokal and Rohlf, 1969), we can sum the chi-square values and degrees of freedom to obtain a total chi-square of 247.8 with 90 degrees of freedom. The probability of obtaining a chi-square value as large or larger is less than 0.001 given the null hypothesis of randomness.

Seven of the thirty chi-square values for $1 / 4$ aliquots were significantly different than expected at the 0.05 level. The expected number was $1.5(=30 \times 0.05)$, and the probability of observing seven or more significant values is less than 0.001 . The total chi-square for the $1 / 4$ aliquots was 130.6 with 70 degrees of freedom, and this value has a probability of less than 0.001 under the assumption of randomness.

Of the three chi-square values for the $1 / 16$ aliquots, none were significantly different from the expected at the 0.05 level. The total chi-square was 14.5 with 15 degrees of freedom and was not significantly different from the random expectation. 
Figure 3.5. Observed and expected standard deviations of the $1 / 2$ aliquot data as a function of the total sample count $\left(\mathrm{N}_{\mathrm{T}}\right)$. 


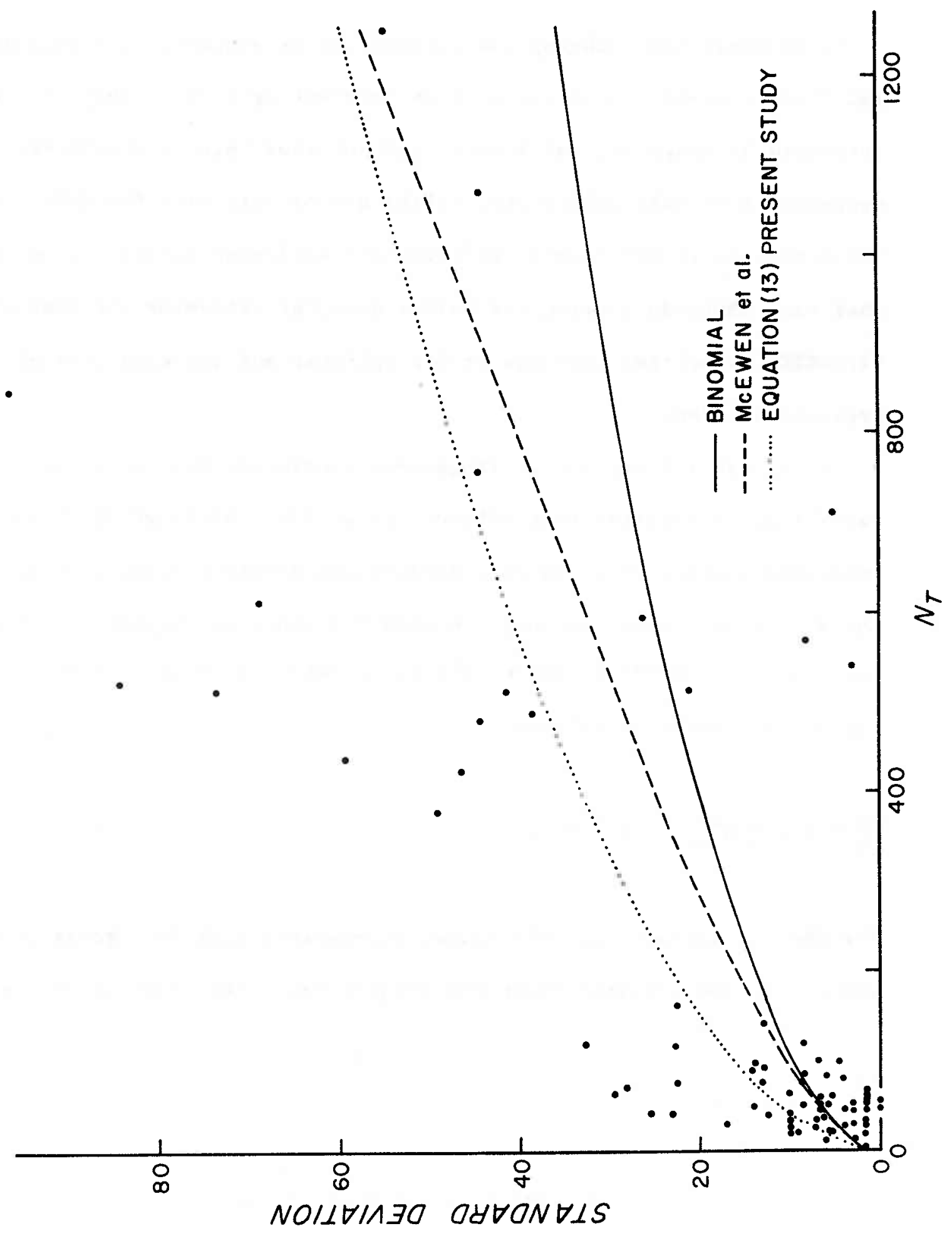


Discussion

It is clear that subsampling variability is nonrandom for both my data and those presented by Miller. It is interesting to note that the data presented by Longhurst and Siebert did not show this nonrandomness. Miller suggested that subsampling variability may be different for different organisms, different Folsom splitters and different workers. I recommend that each individual using the Folsom splitter determine the subsampling variation associated with his or her splitter and for each type of organism counted.

Given that subsampling is in general nonrandom, how can we estimate the variability associated with subsampling in the present study? Miller suggested a means of estimating subsampling variance based upon the chi-square statistic. Although he used the wrong chi-square statistic, the basic idea presented is sound. The chi-square statistic from equation (4) can be rearranged as follows:

$s_{a}^{2}=N \cdot p \cdot q \cdot x_{(n-1)}^{2} /(n-1)$

For the $1 / 2$ aliquots in this study, chi-squared with 90 degrees of freedom was 247.8 . The variance about the aliquot count may be estimated by:

$s_{c_{2}}^{2}=2.75 \cdot \mathrm{N} \cdot \mathrm{p} \cdot \mathrm{q}$

The subsampling variance about $N$, the total sample count, is: 
$s_{N}^{2}=2.75 \cdot N \cdot\left(2^{m}-1\right)$

This equation should be an adequate measure of the subsampling variance as long as the chi-square values are independent of the total sample count. In other words, the chi-square values should not increase (or decrease) as the sample count $(N)$ increases. The correlation coefficient for the chi-square values and total sample counts for the $1 / 2$ aliquots was 0.188 , a value not significantly different from zero.

Calculation of the $x^{2} /(n-1)$ factor from the $1 / 4$ aliquot data yielded a value of 1.87 , which was smaller than that calculated from the $1 / 2$ aliquot data. Because most of my data are included in the calculation of the first factor (2.75), I will assume that its value is appropriate for the subsampling performed during the course of my work. The standard deviations associated with the estimate of subsampling variance from equation (13), with the binomial estimate of subsampling variance, and with the McEwen et al. estimate of subsampling variance are included in Figures 3.5. It appears that the estimate from equation (13) was the best predictor of variance for these data.

Given that subsampling is necessary at times, what aliquot size is needed to minimize the subsampling variability and to reduce the time and effort needed to sort plankton samples to a manageable level. Equation (13) may be manipulated so that the coefficient of variation can be calculated as a function of the abundance of the organisms in the sample and the aliquot size:

$\mathrm{s}_{\mathrm{N}} / \mathrm{N}=\left(2.75 \cdot\left(2^{\mathrm{m}}-1\right) / \mathrm{N}\right)^{1 / 2}$ 
The coefficient of variation is plotted as a function of the total sample count for four aliquot sizes in Figure 3.6. These graphs indicate that it is inefficient to count more than 50 to 100 individuals in each aliquot. Beyond aliquot counts of 100, a large increase in the number of individuals counted is needed to produce even a small decrease in the coefficient of variation. If the level of variability introduced by subsampling is acceptable, then the sample should be split such that between 50 and 100 individuals of each of the organisms in question are present in the final aliquot. This may require different aliquot sizes for different organisms from the same sample.

For my work, I subsampled by splitting a sample until there were approximately 300 to 500 individuals of all species combined. Although it would have been best to count different aliquot sizes based on the abundance of each of the species in the sample, this was not found to be practical. For rare species the entire sample would have to be sorted, but each chaetognath in the sample would have to be examined in order to determine if it was a member of the rare species. For many samples this would mean looking at over 10,000 specimens, a number which would take far too much time to examine.

Given the aliquot count and aliquot size, we can now determine the variance associated with that aliquot count. The subsampling variance can be compared with the variance expected from small scale patchiness, and hopefully its value will be small relative to that due to patchiness. The variance due to small scale patchiness can be calculated from the equation suggested by Cassie (1968) (see patchiness section): 
Figure 3.6. Coefficient of variation as a function of aliquot count (C) and total sample count $\left(\mathrm{N}_{\mathrm{T}}\right)$ for $1 / 2,1 / 4,1 / 8$, and $1 / 16$ aliquots. 

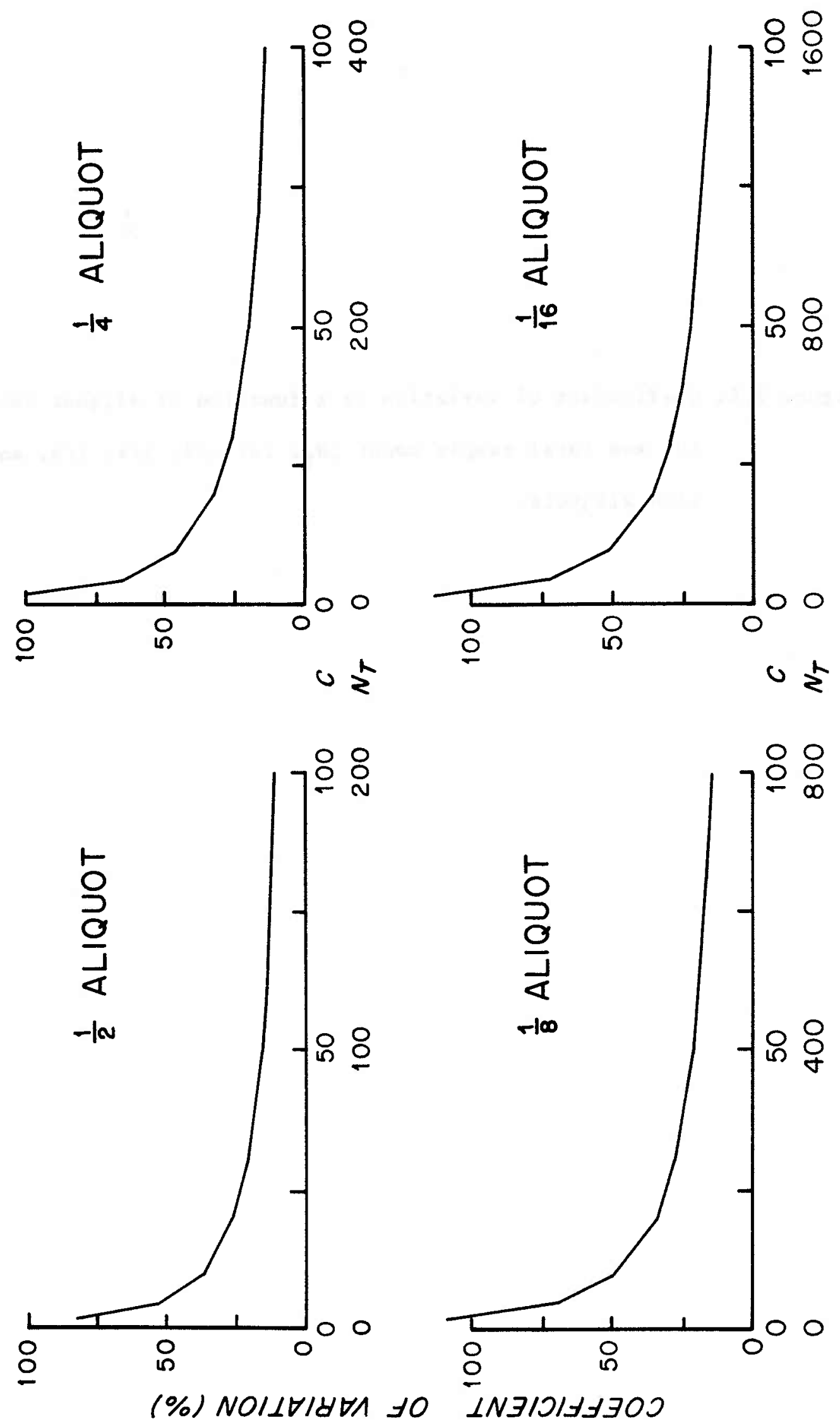
$s_{p}^{2}=u+c \cdot u^{2}$

Cassie (1968) quotes a range from 0.05 to 0.10 for the coefficient $c$ from previous studies and analysis of the two horizontal tows in the earlier section on patchiness suggested that the value of $c$ for chaetognaths in this study was somewhere between 0.081 and 0.200 . I will assume a value of 0.10 for the chaetognaths in this study because it is within the ranges just quoted. Thus the variance due to small scale patchiness can be calculated given the total number of chaetognaths in the sample.

A comprehensive comparison of variability due to patchiness and subsampling would require analysis of seventeen chaetognath species in each sample (eight samples per tow and 38 tows) because the aliquot size and total number for each species varies from sample to sample. An analysis which provides almost as much information and requires much less time can be made for average abundances of chaetognaths in these samples and average aliquot sizes for the samples in which these species occur. Because the abundance of each of these species and the aliquot size were quite different in samples from the SW and NSS, I will compare the subsampling and small scale patchiness variability for each of these species in each of these two hydrographic regions.

We first need an estimate of the total numbers of each of these species in each of the MOC 1 tows. The original sample counts have all been converted to numbers per $1000 \mathrm{~m}^{3}$ and these values have been integrated for each tow to provide numbers under a square meter of ocean surface. Since most of the tows sampled the upper $1000 \mathrm{~m}$ of the water column, the numbers per $\mathrm{m}^{2}$ of ocean surface also represent the average numbers per 
$1000 \mathrm{~m}^{3}$ in the upper $1000 \mathrm{~m}$. The average volume of water filtered by all the nets during a tow was approximately $5000 \mathrm{~m}^{3}$. The total numbers of chaetognaths in each tow can therefore be calculated by multiplying the number per $\mathrm{m}^{2}$ by 5.0 . The median abundance per $\mathrm{m}^{2}$ for each species in each of the two hydrographic regions was multiplied by 5.0 to estimate the average numbers caught in each tow.

We must also determine the average aliquot sizes for each of the depth intervals sampled. I have estimated the average aliquot size for each of the depth intervals by summing the number of splits for each depth interval over all the tows in a given hydrographic region and dividing this sum by the number of tows. I will use depth interval 4 as an example ( 1 =deepest sample, $8=$ shallowest samples). In the SW tows, no aliquots were taken in this depth interval in any of the samples, so the sum of the splits is zero and the average number of splits was zero. For the Northern Sargasso Sea tows, $1 / 2$ aliquots ( 1 split) were counted for three samples, $1 / 4$ aliquots ( 2 splits) were taken for one sample, and for the other sixteen tows the entire samples were counted. The sum of the number of splits for this depth interval is $3 \times 1+1 \times 2+16 \times 0=5$. The average number of splits for this depth interval is $5 / 20=0.25$. The average number of splits for all depth intervals calculated in this manner is shown in Table 3.14 .

Most of the chaetognaths in these tows occur in more than one depth interval, so an average split number for each species was obtained by averaging the split numbers from Table 3.14 over the range of depth intervals occupied by each species (Table 3.15 ).

We have now estimated the average number of each species per tow and 
Table 3.14. Average number of splits for the eight depth intervals from the Slope Water and Northern Sargasso Sea. 


\begin{tabular}{|c|c|c|c|c|}
\hline Hydrographic region & Net非 & Sum of splits & \# of tows & Average splits \\
\hline \multirow[t]{8}{*}{ Slope Water } & 1 & 0 & 18 & 0 \\
\hline & 2 & 0 & 18 & 0 \\
\hline & 3 & 0 & 18 & 0 \\
\hline & 4 & 0 & 18 & 0 \\
\hline & 5 & 0 & 18 & 0 \\
\hline & 6 & 2 & 18 & 0.11 \\
\hline & 7 & 9 & 18 & 0.50 \\
\hline & 8 & 39 & 17 & 2.17 \\
\hline Northern Sargasso & 1 & 0 & 20 & 0 \\
\hline \multirow[t]{7}{*}{ Sea } & 2 & 0 & 20 & 0 \\
\hline & 3 & 1 & 20 & 0.05 \\
\hline & 4 & 5 & 20 & 0.25 \\
\hline & 5 & 11 & 20 & 0.55 \\
\hline & 6 & 16 & 20 & 0.80 \\
\hline & 7 & 42 & 20 & 2.10 \\
\hline & 8 & 50 & 20 & 3.00 \\
\hline
\end{tabular}


the average number of splits for each species in each of these two hydrographic regions. Table 3.15 presents the standard deviations due to subsampling $\left(s_{s}\right)$ and patchiness $\left(s_{p}\right)$ for seventeen chaetognath species from these samples. In some cases, especially for epipelagic species, the subsampling variability was greater than that due to small scale patchiness. It should be noted, however, that for 27 of the 34 comparisons, the variablity due to patchiness was greater than that due to subsampling, and that for 20 comparisons the subsampling standard deviation was less than half that for patchiness. While subsampling appears to be a problem for some of these species, it appears not to be a major problem for most of them. My advice is to keep subsampling to a minimum, and if subsampling is necessary, one must be cautious in the interpretation of the sources of the observed variability in abundance. 


\section{Table 3.15. Comparison of variability due to subsampling $\left(s_{s}\right)$ and variability due to small scale patchiness $\left(s_{p}\right)$ for seventeen chaetognath species.}


SLOPE WATER

\begin{tabular}{llllll} 
& $\begin{array}{l}\text { Median } \# \\
\text { Species }\end{array}$ & Mean tow & & \\
& of splits & $s_{s}$ & $s_{p}$ & $s_{s} / s_{p}$ \\
\hline
\end{tabular}

Bathypelagic species

$\begin{array}{lcllll}\text { E. bathypelagica } & 9.5 & 0 & 0 & 4.30 & 0 \\ \text { E. fowleri } & 30.0 & 0 & 0 & 10.95 & 0 \\ \text { E. hamata } & 160 & 0.08 & 5.01 & 52.15 & 0.10 \\ \text { S. macrocephala } & 200 & 0 & 0 & 64.81 & 0 \\ \text { S. maxima } & 90.0 & 0.09 & 3.99 & 30.00 & 0.13\end{array}$

Mesopelagic species

$\begin{array}{lrrrrr}\text { K. subtilis } & 13.5 & 0.40 & 3.44 & 5.63 & 0.61 \\ \text { S. decipiens } & 65.0 & 0.15 & 4.43 & 22.08 & 0.20 \\ \text { S. lyra } & 37.0 & 0.46 & 6.18 & 13.19 & 0.47 \\ \text { S. planctonis } & 9.0 & 0.02 & 0.59 & 4.14 & 0.14\end{array}$

Epipelagic species

$\begin{array}{lccccr}\text { K. pacifica } & 12.0 & 2.2 & 10.89 & 5.14 & 2.12 \\ \text { P. draco } & 8.0 & 2.2 & 8.89 & 3.79 & 2.35 \\ \text { S. bipunctata } & 6.0 & 2.2 & 7.70 & 3.10 & 2.48 \\ \text { S. enflata } & 650 & 2.2 & 80.16 & 207.1 & 0.39 \\ \text { S. hexaptera } & 9.0 & 1.3 & 6.02 & 4.14 & 1.45 \\ \text { S. minima } & 155 & 2.2 & 39.14 & 50.57 & 0.77 \\ \text { S. serratodentata } & 33.0 & 1.3 & 11.52 & 11.91 & 0.97 \\ \text { S. tasmanica } & 1275 & 1.3 & 71.60 & 404.8 & 0.18\end{array}$


Table 3.15. (cont'd).

NORTHERN SARGASSO SEA

$\begin{array}{llllll}\text { Median } & \text { Mean } \#_{1} & & & \\ \text { per tow } & \text { of splits } & s_{s} & s_{p} & s_{s} / s_{p}\end{array}$

$\begin{array}{lccccc}\text { E.b. } & 0.10 & 0 & 0 & 0.32 & 0 \\ \text { E.f. } & 13.0 & 0 & 0 & 5.47 & 0 \\ \text { E.h. } & 3.2 & 0 & 0 & 2.06 & 0 \\ \text { S.m. } & 19.5 & 0 & 0 & 7.58 & 0 \\ \text { S.m. } & 0.90 & 0 & 0 & 0.99 & 0\end{array}$

$\begin{array}{llllll}\text { ik.s. } & 535 & 0.96 & 53.50 & 170.8 & 0.31 \\ \text { S.d. } & 735 & 0.96 & 43.71 & 234.0 & 0.19 \\ \text { S.1. } & 665 & 1.1 & 45.73 & 211.9 & 0.22 \\ \text { S.p. } & 33.5 & 0.84 & 5.41 & 12.07 & 0.45\end{array}$

$\begin{array}{lccccc}\text { K.p. } & 34.0 & 3.0 & 25.58 & 12.23 & 2.09 \\ \text { P.d. } & 635 & 3.0 & 110.6 & 202.4 & 0.55 \\ \text { S.b. } & 145 & 3.0 & 52.83 & 47.41 & 1.11 \\ \text { S.e. } & 210 & 2.6 & 54.07 & 67.97 & 0.80 \\ \text { s.h. } & 360 & 2.0 & 54.50 & 115.4 & 0.47 \\ \text { s.m. } & 660 & 2.6 & 95.86 & 210.3 & 0.46 \\ \text { s.s. } & 575 & 3.0 & 105.2 & 183.4 & 0.57 \\ \text { s.t. } & - & - & - & - & - \\ \end{array}$


F. Miscellaneous

Several other error sources were identified for the chaetognath abundance estimates from this study. They appear to be minor errors compared with those discussed in the preceding pages.

In the course of my thesis work, I examined 103,168 chaetognaths of which I was able to identify 94,667 (91.8\%). Most of the unidentified individuals were either damaged beyond recognition or were juveniles lacking the diagnostic characters needed for identification.

The presence of unidentified specimens results in an underestimate of the abundance of chaetognaths in nature. If the percentage of unidentified specimens was the same for all species, the species counts could be multiplied by $1.089(1 / .918)$ for this study to correct for this source of error. The percentage unidentified was not, however, equally distributed among the species, nor was the percentage unidentified the same for all the tows. The range of the percent unidentified in the tows was from 2.9 to $20.1 \%$. This value probably depends on such factors as the speed of the tow (which affects the damage to the catch), the proportions of the different species in the water and the population size structure for individual species (more juveniles result in more unidentified specimens).

The percent unidentified was also not distributed equally among the species. Of the unidentified specimens, $49.0 \%$ were Eukrohnia spp., while the genus Eukrohnia accounted for only $11.2 \%$ of the total number of chaetognaths examined in this study. Unidentified specimens accounted for $35.9 \%$ of all Eukrohnia specimens, while on 1 y $4.7 \%$ of the remaining species were unidentified. Most of the Eukrohnia spp. were headless 
juveniles, and I believe that they were primarily E. fowleri. The small fraction of the remaining species which were not identified was insignificant in light of the other sources of error in this study. Zooplankters, at times, may become caught and entangled in the meshes of the net. Spinose forms are most likely to be trapped in the meshes, and some organisms, such as chaetognaths, may actively grab and hold onto the net gauze. Net hanups by chaetognaths are an important source of sampling bias for the Longhurst-hardy Plankton Recorder (Haury, 1976). Haury found that chaetognaths and polychaetes tend to hangup during the tow, but after the LHPR surfaced, they were washed into the recorder box. For the MOC 1 samples, the nets were thoroughly washed down, and this should have effectively washed all the chaetognaths into the cod end buckets. When specimens are not washed into the cod end buckets, they become dried out as the net material dries between tows. Dried specimens take on a very flattened and shiny appearance, and they are readily detectable as they are washed into the cod end bucket during the next tow. I observed fewer than $0.05 \%$ of the individuals in these samples with this appearance. Misidentification may result in underestimation of the abundance of a species which is misidentified as another species and overestimation of the abundance for the species to which individuals were wrongly assigned. Because there was little problem with identification in this study, this source of sampling error should have little or no effect for the MOC 1 samples. Specimens for which misidentification might have been a problem were usually placed in the unidentified category. During the analysis of size frequency for S. 1yra, I discovered that two S. maxima and one S. $\underline{\text { hexaptera }}$ were misidentified as $\underline{S}$. lyra. Although this undoubtedly does 
not include all those specimens which were misidentified as $\underline{S}$. Iyra, the low number of misidentifications found does not pose a serious problem in the estimation of chaetognath abundance. 
CHAPTER 4

Vertical Distribution of Chaetognaths

in the Western North Atlantic Ocean 
Introduction

There is a voluminous literature documenting the vertical distributions of marine zooplankton (see Vinogradov, 1970 for an extensive review), but very little is known about how these organisms select their vertical positions in the water column. Environmental conditions change rapidly with depth in the ocean, so successful growth and reproduction depend upon the depths at which an organism lives. We need to understand how this vertical habitat selection occurs if we are to understand how the horizontal and temporal abundance patterns of zooplankton are regulated.

Broad vertical distribution patterns are known for most chaetognaths (Alvarino, 1965), yet little is known of the factors regulating these patterns. While laboratory studies have provided some insight into the mechanisms involved (Singarajah, 1966; Harder, 1968), we have had to rely primarily upon comparisons of vertical distributions in the field with variables such as temperature, light and salinity. The strong degree of correlation among these variables and the lack of adequate sampling techniques in the past have made it difficult to assess their effects. Kecent advances in the development of multiple opening/closing nets and concomitant measurement of environmental variables such as temperature, salinity, and oxygeri concentration as a function of depth enable us more accurately to describe the vertical distributions and their relationships with these variables (Frost and McCrone, 1974; Wiebe et al., 1976a; Sameoto et al., 1977). In addition, sampling in regions where there are substantial horizontal differences in the values of these variables at a given depth allow the uncoupling of correlations among them. Such an area 
is found in the western North Atlantic Ocean south of Cape Cod, Massachusetts. Vertical profiles of temperature, salinity, and oxygen concentration are quite different in the Slope Water (hereafter referred to as SW) to the nortin of the Gulf Stream (GS) and the Northern Sargasso Sea (NSS) to the south (Figure 4.1). Gulf Stream cold core rings, formed by the pinching off of Gulf Stream meanders to the south, may be used as quasi-experimental sites where the correlations between many important variables change with time (Wiebe et al., 1976b). The vertical distributions of chaetognaths have not been studied in this region, although there have been several studies in adjacent regions [Bigelow (1926), Huntsman (1919), and Aurich (1971) to the north and northeast; Moore (1949) in the Northern Sargasso Sea off Bermuda; and Owre (1960) in the Florida Current off Miami].

This study was undertaken to describe the average vertical distributions and diel and ontogenetic vertical migrations of chaetognaths in the upper $1000 \mathrm{~m}$ of the SW and NSS. Because temperature and light have been reported to control the vertical distributions of several chaetognaths (Russe11, 1931; Moore et a1., 1953; Owre, 1960), vertical distributions were compared with temperature and light profiles from 52 MOCNESS tows. During the course of this analysis, however, it was discovered that vertical distribution as measured by median depth of the total sampled population was inadequate for assessing the effects of temperature and light. Ontogenetic migrations combined with spatial or temporal changes in the size frequency distribution of many of these chaetognaths may be responsible for the observed variations in median depth in this study and in comparisons with other studies. 


\author{
Figure 4.1. Hydrographic comparisons of the upper $1000 \mathrm{~m}$ of the \\ Slope Water and Northern Sargasso Sea. \\ a. Temperature envelopes- MOC $1-91$ and 115 represent \\ the Gulf Stream and MOC 1-60,61 represent the NSS in \\ April 1977. \\ b. Salinity envelopes- MOC 1-60,61 represent the NSS in \\ Apri1 1977. \\ c. Oxygen envelopes- Redrawn after Wiebe et al. (1976b). \\ d. Noon light envelopes- See text for methods used in \\ calculation.
}




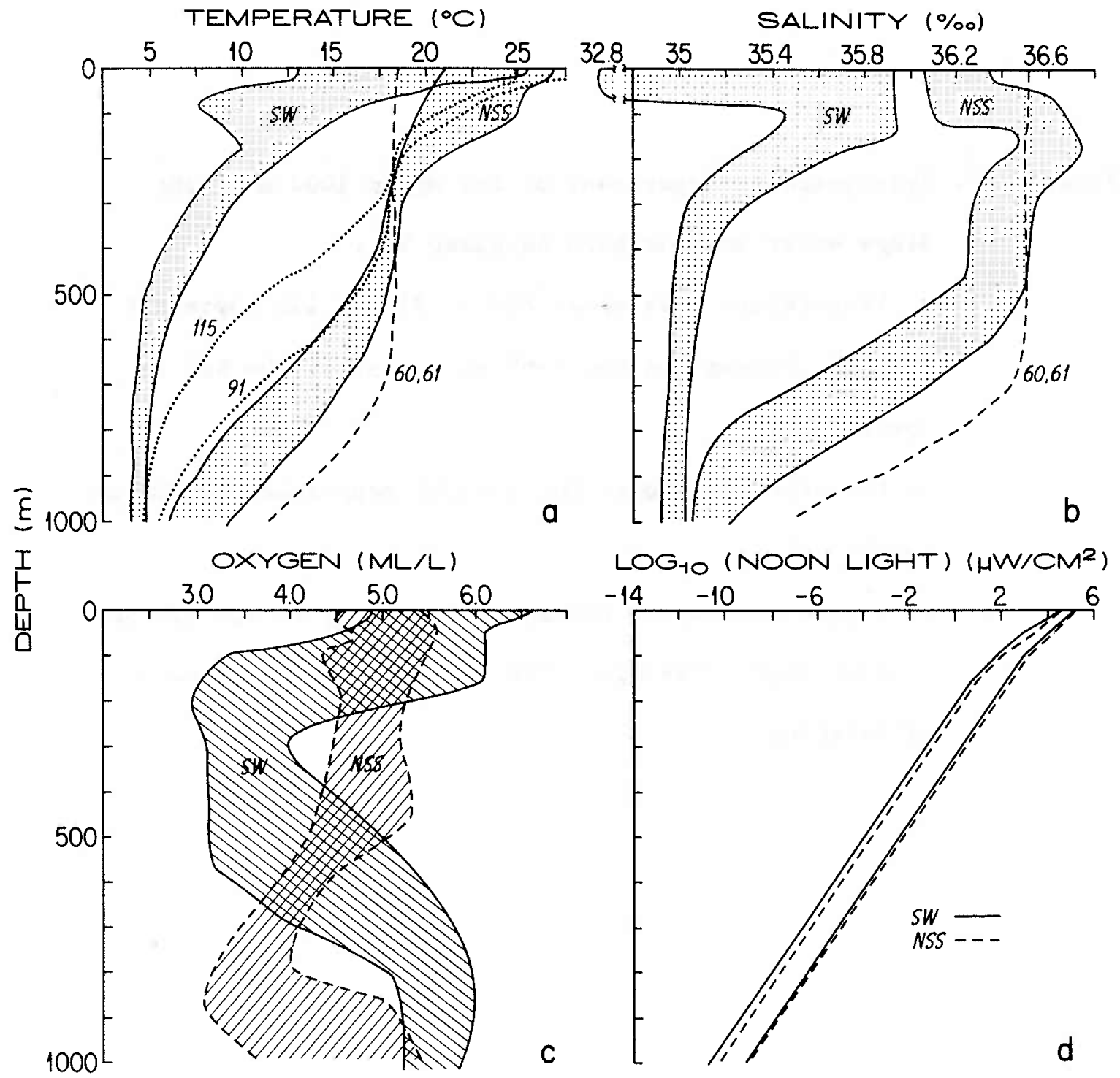


Methods

Plankton samples were collected and analyzed as discussed in Chapter 2. Because the depth intervals sampled varied somewhat between tows, all vertical distributions were converted to $100 \mathrm{~m}$ depth intervals from the surface to $1000 \mathrm{~m}$ to facilitate comparisons between tows. For any given $100 \mathrm{~m}$ depth interval, this was done by summing the product of the number per $1000 \mathrm{~m}^{3}$ and the fraction of that $100 \mathrm{~m}$ depth interval contributed by each of the eight original depth intervals. Such a procedure resolves the upper $1000 \mathrm{~m}$ into ten depth intervals where in fact only eight were sampled, but this manipulation had little or no effect on subsequent calculations of median depth.

The average vertical distribution for each species was determined separately for the SW and NSS because the physical structure of the water column varied greatly between these regions and observations of individual tows showed greatest differences in vertical distributions between these two hydrographic regions. Average number per $1000 \mathrm{~m}^{3}$ for each $100 \mathrm{~m}$ depth interval was calculated using the eighteen SW and eighteen NSS tows (Table 2.1).

Analysis of the diel and ontogenetic vertical migrations and relation of vertical distributions to environmental variables ideally requires the capaility to detect significant differences in vertical distribution between tows. The vertical distribution data in this study was represented by abundance values within each of eight depth intervals. Ordinarily, 
either the chi-square goodness of fit or the Kolmogorov-Smirnov test would be applied to such data, but both procedures test for departures greater than expected at random. The analysis of patchiness in Chapter 3 showed that the small scale horizontal abundance patterns for these chaetognaths were in fact nonrandom. Thus the number of organisms within each depth interval varied nonrandomly and either of the previously mentioned tests would yield significant differences between almost any two tows. For example, when the Kolmogorov-Smirnov test was applied to the vertical distributions for $\underline{S}$. decipiens in each of fourteen day/night tow pairs, nine pairs were significantly different at the 0.05 level. S. decipiens did not vertically migrate on a daily basis (see Results), so little or no difference was expected between the members of a tow pair. The probability of obtaining nine or more significant differences at the 0.05 level given the null hypothesis of no real differences is less than 0.001 . Analysis of differences in vertical distribution between individual tows will have to await the development of a statistical procedure which takes into account the nonrandom horizontal variations for these species.

In the absence of such a statistical technique, I chose to use the median depth as a measure of vertical distribution in tests for differences in vertical distribution between SW and NSS, for diel vertical migration, and for correlating vertical distributions with environmental conditions. Median or mean depths have been used by others for such tests (Moore, 1949; Moore et a1., 1953; Owre, 1960; Pearre, 1974). The median depth is defined as the depth above and below which $50 \%$ of the population occurs. It was calculated by first determining the cumulative percent of the population within each depth interval from the surface down. The depth 
of the 50th cunulative percentile was then computed assuming a uniform distribution within the depth interval encompassing the 50 th cumulative percentile.

Temperature as a function of depth was determined from plots generated from the MOCNESS CTD during each of the tows. The depth of the mixed layer was also determined from these plots for correlation with the vertical distribution of the epipelagic species. Light was not measured directly during MOCNESS tows, so it was calculated as a function of depth by an indirect method. Noon solar irradiation at the sea surface was calculated for the latitude and date of each tow using Lumb's (1964) formula as presented by Reed (1977) and was corrected for cloudiness using the formula presented by Reed (1977). The amount of downwelling light at depth was calculated in $\mathrm{uW} / \mathrm{cm}^{2}$ from the equation:

$I_{z}=I_{0} e^{-k \cdot z}$

where $I_{z}$ is the light at depth $z, I_{0}$ is the noon surface irradiation corrected for cloudiness, and $\mathrm{k}$ is the extinction coefficient. Penetration of light of different wavelengths could not be calculated because of the absence of data on the spectral composition of the surface light and the lack of wavelength dependent extinction coefficients. The wavelengths of light to which chaetognaths are sensitive are also unknown. Extinction coefficients were calculated from an equation presented by Riley (1950), which assumes that chlorophyll a is the only material, besides the water itself, responsible for absorbing and scattering light. This equation was modified by using a value of 0.03 rather than 0.04 for the extinction 
coefficient of clear oceanic water because it is more representative of the values obtained by subsequent investigators (Jerlov, 1976).

Chlorophyl1 a profiles in the upper $200 \mathrm{~m}$ were obtained in conjunction with most of the MOCNESS tows. The extinction coefficient for each tow was allowed to vary as a function of the measured chlorophyll a changes with depth in the upper $200 \mathrm{~m}$, and chlorophyll a was assumed to be absent below $200 \mathrm{~m}$.

Direct correlation of median depth with temperature or light at that depth was inappropriate because the expected correlation coefficient would be zero if these chaetognaths were following isotherms or isolumes. Median depth can be correlated with the depth of a particular isotherm or isolume as was done by Moore et al. (1953) or with the value of temperature or light at a specified depth. Either method should yield similar results. I have chosen to correlate median depth with temperature and light at a specified depth because it was the most convenient method.

Because of the extent of the ontogenetic migrations of some of these species (see Results), it appeared that differences in median depth between tows might be a function of changes in population size Erequency distribution rather than responses to changes in the depths of specific environmental conditions. Size frequency distribution was measured only for S. Iyra in all the tows, so I also correlated median depth of this species with average individual size for each tow. Product-moment correlation coefficients were used exclusively. 
Resu1ts

Average vertical distributions

Twenty one chaetognath species were identified from these tows. Data concerning three species collected in insufficient quantities for adequate assessment of their vertical distributions are presented in Table 4.1 . S. $\underline{\text { elegans }}$ and $\underline{\mathrm{S}}$ hispida are both reported to be epipelagic coastal species

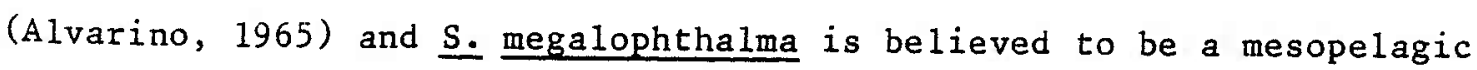
species (Dallot and Ducret, 1969). The limited observations reported here do not conflict with their previously reported vertical distributions. Average vertical distributions for the remaining eighteen species in the SW and NSS are presented in Figure 4.2. These species may be broadly classified from this study and previous reports as epipelagic $(0-200 \mathrm{~m})-$ K. pacifica, P. draco, S. bipunctata, S. enflata, S. helenae, S. hexaptera, S. minima, S. serratodentata, and S. tasmanica; mesopelagic (200-1000 m)- K. subtilis, S. decipiens, S. 1yra, and S. planctonis; and bathypelagic (below $1000 \mathrm{~m}$ ) - E. bathypelagica, E. fowleri, E. hamata, S. macrocephala, and S. maxima. It is clear that the meso- and bathypelagic species do not fit well into the idealized 200-1000 m and greater than $1000 \mathrm{~m}$ categories, respectively. Most of the species which were bathypelagic in the NSS were mesopelagic in the SW. The terms are used here rather loosely to denote species with relatively similar vertical distribution patterns and which occur primarily in the depth ranges cited. Judging from the shapes of the vertical distributions, the populations of

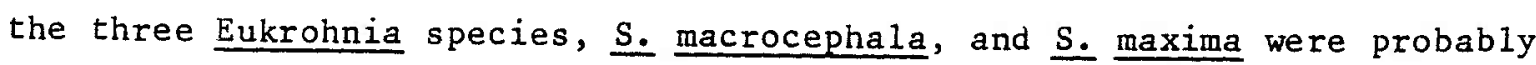


Table 4.1. Records of occurrence of rare chaetognath species in this study. 


\begin{tabular}{|c|c|c|c|c|}
\hline Species & $\begin{array}{c}\text { Hydrographic } \\
\text { Region }\end{array}$ & MOC1非 & $\begin{array}{c}\text { Depth } \\
\text { Interval (m) }\end{array}$ & $\begin{array}{l}\text { Number }{ }_{3} \text { per } \\
1000 \mathrm{~m}^{-}\end{array}$ \\
\hline \multirow[t]{8}{*}{ S. elegans } & SW & 116 & $0-50$ & 23.2 \\
\hline & & & $50-100$ & 21.7 \\
\hline & & & $100-200$ & 6.2 \\
\hline & & 117 & $50-100$ & 45.1 \\
\hline & & & $100-200$ & 4.2 \\
\hline & & & $200-300$ & 4.2 \\
\hline & & & $300-400$ & 3.0 \\
\hline & & & $400-600$ & 1.0 \\
\hline S. hispida & SW & 75 & $0-100$ & 15.1 \\
\hline \multirow[t]{3}{*}{ S. megalophthalma } & NSS & 65 & $400-550$ & 2.9 \\
\hline & GS & 115 & $0-100$ & 12.3 \\
\hline & & & $300-400$ & 3.9 \\
\hline
\end{tabular}


Figure 4.2. Average vertical distributions of chaetognaths in the upper $1000 \mathrm{~m}$ of the Slope Water and Northern Sargasso Sea. Arrows indicate median depths calculated from the average vertical distributions. Depth intervals labeled with asterisks contained less than $1 \%$ of the population. 


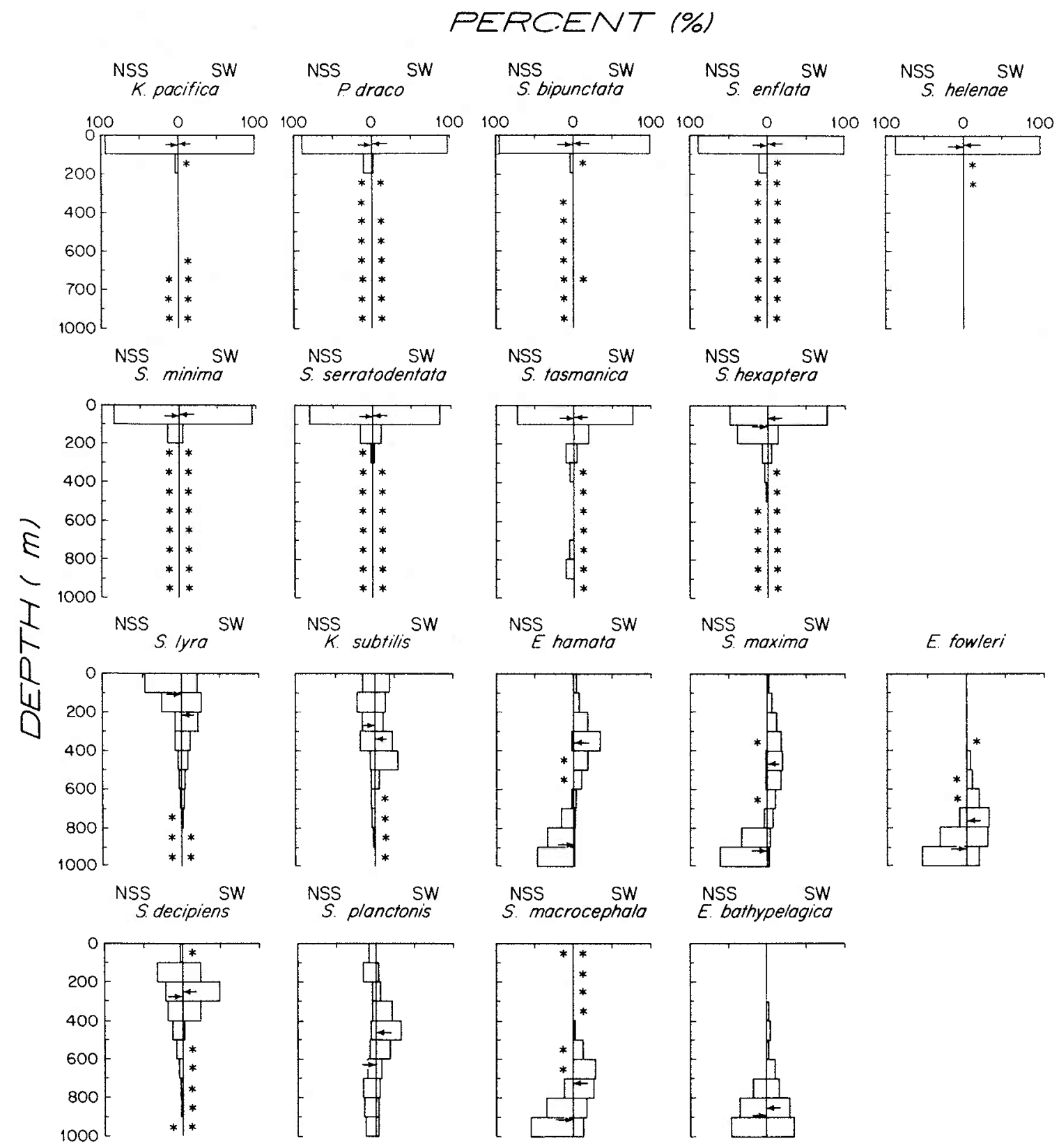


present in susbstantial numbers below $1000 \mathrm{~m}$ in the NSS (thus their classification as bathypelagic species). Substantial proportions of the populations of E. bathypelagica, E. fowleri and S. macrocephala were also probably present below $1000 \mathrm{~m}$ in the SW. The presence of individuals of the epipelagic species below depths of $300-400 \mathrm{~m}$ probably resulted from either contamination or capture of sinking, moribund individuals.

The vertical distributions of the three Eukrohnia species should be viewed with caution as a large fraction ( $36 \%$ ) of the specimens of this genus could not be identified. Most of the unidentified specimens appeared to be juvenile E. fowleri, so the vertical distributions presented for $\underline{E}$. bathypelagica and $\underline{E}$. hamata are probably accurate.

Table 4.2 presents a statistical comparison of median depths in the SW and NSS for the species from Figure 4.2. Mean median depths calculated in Table 4.2 were generally in agreement with median depths calculated from the average vertical distributions presented in Figure 4.2. Differences in values for the median depth were due to the different procedures for calculation. Mean median depths presented in Table 4.2 give equal weight to each tow, while median depths calculated from the average vertical distributions in Figure 4.2 are weighted by the abundance of each species in each tow. Observed differences were rarely greater than $15 \mathrm{~m}$ (three exceptions) for the epipelagic species and $40 \mathrm{~m}$ (two exceptions) for the meso- and bathypelagic species. It should be noted that given the depth intervals sampled, 50 and $925 \mathrm{~m}$ were the minimum and maximum values obtainable for median depth in this study. The ranges given in Table 4.2 indicate that variability in median depth can be quite large for most of these species. 
Table 4.2. Comparison of median depths in the SW and NSS.

Differences between regions were tested using the Mann-Whitney U test. 
Bathypelagic species

$\begin{array}{lrllllll}\text { E. bathypelagica } & 9 & 775 * & 488-891 & 2 & 848 & 775-920 & \text { NS } \\ \text { E. fowleri } & 8 & 770 & 715-829 & 13 & 909 & 828-925 & \text { p }<0.01 \\ \text { E. hamata } & 14 & 359 & 160-455 & 13 & 871 & 775-925 & \mathrm{p}<0.01 \\ \text { S. macrocepha1a } & 9 & 703 & 647-776 & 14 & 907 & 834-925 & \mathrm{p}<0.01 \\ \text { S. maxima } & 14 & 452 & 338-670 & 10 & 767 * & 475-925 & \mathrm{p}<0.01\end{array}$

Mesopelagic species

$\begin{array}{lllrlrrc}\text { K. subtilis } & 14 & 363 & 79-456 & 17 & 269 & 135-387 & \mathrm{p}<0.01 \\ \text { S. decipiens } & 16 & 275 & 218-352 & 18 & 272 & 171-428 & \text { NS } \\ \text { S. Iyra } & 14 & 249 & 74-375 & 18 & 125 & 63-352 & \mathrm{p}<0.01 \\ \text { S. planctonis } & 15 & 462 & 230-685 & 13 & 647 & 105-896 & \mathrm{p}<0.01\end{array}$

Epipelagic species

\begin{tabular}{lccccccc} 
K. pacifica & 11 & 60 & $50-164$ & 15 & 52 & $50-60$ & NS \\
P. draco & 11 & 52 & $50-67$ & 18 & 60 & $50-111$ & $\mathrm{p}<0.01$ \\
S. bipunctata & 10 & 50 & $50-100$ & 17 & 61 & $50-135$ & $\mathrm{p}<0.05$ \\
S. enflata & 16 & 51 & $50-62$ & 18 & 62 & $51-104$ & $\mathrm{p}<0.01$ \\
S. hexaptera & 15 & $112^{*}$ & $53-218$ & 18 & 105 & $65-163$ & NS \\
S. minima & 13 & 55 & $50-87$ & 18 & 64 & $51-114$ & $\mathrm{p}<0.05$ \\
S. serratodentata & 15 & $111^{*}$ & $50-273$ & 18 & 61 & $51-83$ & NS \\
S. tasmanica & 17 & 80 & $50-197$ & 4 & $356 *$ & $50-775$ & NS \\
& & & & & & & \\
\hline
\end{tabular}

* Median depths which differ substantially from median depths presented in Figure 4.2. (see text for discussion). 
Comparisons of the vertical distributions in the SW and NSS can be used in a preliminary analysis of how these species select their vertical distributions. The effects of biotic factors such as prey and predator distributions could not be assessed because information on their vertical distributions was not available. The values of salinity and oxygen concentration (Figure 4.1) were probably not limiting for any of the species considered here. If a species orients its vertical distribution to a particular isotherm, then it should migrate upward in the SW as compared with the NSS. If a species orients its vertical distribution to light or pressure, there should be little or no difference in vertical distribution between the two regions (Figure 4.1). A note of caution should be issued here- many of the meso- and bathypelagic species exhibited large ontogenetic migrations, so the observed vertical distributions may be a function of the size frequency distribution as well as behavioral responses to these physico-chemical variables. This will be discussed more fully in later sections. In general, species within each broadly defined depth category (epi-, meso-, and bathypelagic) behaved in a similar manner.

For each of the nine epipelagic species, the fraction of the population below $100 \mathrm{~m}$ was smaller in the SW than the NSS (Figure 4.2). This is indicated by their shallower median depths in the SW. Four of these species were significantly shallower in the $\mathrm{SW}$, while the remaining four showed no significant differences (Table 4.2). S. helenae was not included in Table 4.2 because it was not abundant enough for adequate analysis. Mean median depth for S. serratodentata (Table 4.2) was deeper in the SW due to the presence of a few individuals in several deep SW samples. Although $\underline{S}$. tasmanica showed a large difference in mean median depth 
between SW and NSS (Table 4.2), its presence in a small number of tows in the SW and in one tow with a median depth of $50 \mathrm{~m}$ resulted in no significant difference. The results from Figure 4.2 were based upon the behavior of the majority of the individuals sampled, so it is assumed that those results were more representative of the vertical distributions than the results of Table 4.2 and it is concluded that the epipelagic species were all shallower in the SW. Because water temperature in the upper $200 \mathrm{~m}$ was higher in the NSS, it may be argued that low temperature was an important factor limiting the penetration of these species into deeper waters in the $\mathrm{SW}$. Much better vertical resolution in the upper $200 \mathrm{~m}$ is needed to substantiate this hypothesis.

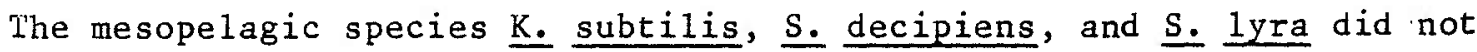
show upward population migrations in the SW. In fact, $\underline{K}_{\text {. subtilis }}$ and $\underline{\mathrm{S} \text {. }}$ lyra were found to be significantly deeper in the SW (Table 4.2). Although it may be argued that $\underline{S}$. decipiens was responding to light or pressure, none of the measured physical factors corresponded with the deeper distributions for the other two species in the SW. As will be discussed later, it is likely that the differences in vertical distribution observed for these two species were due to ontogenetic migrations coupled with population size structure shifts in the SW.

The vertical distribution of $\underline{S}$. planctonis was bimodal in the NSS, suggesting that the NSS population was composed of two subspecies, S. R. $\underline{\text { planctonis }}$ and S. p. zetesios. These subspecies were reported as co-occurring in the Atlantic Ocean by Pierrot-Bults (1975), with $\underline{\text { S. P. }}$

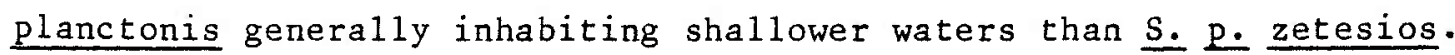
Separation of the subspecies in these tows is needed for further analysis 
of their vertical distributions.

A11 the bathypelagic species except E. bathypelagica showed significant upward shifts in vertical distribution in the SW. This argues for temperature control of their vertical distributions, although, as will be discussed later, these patterns may also result from ontogenetic migrations and population size structure shifts.

Diel vertical migrations

Sixteen day/night paired tows were available for the analysis of diel vertical migrations for seventeen chaetognath species. Fewer than sixteen day/night pairs were actually available for any species because species were sometimes absent from one or both tows of a pair and because occasionally samples were missing in a tow from the depth range normally occupied by a species. S. helenae was not found frequently enough in these tows for adequate statistical analysis of its day/night differences in vertical distribution.

Night median depth was significantly shallower than day median depth only for S. tasmanica (Table 4.3). The probability of obtaining one or more significant differences at the 0.05 level for sixteen tests is 0.51 , so I conclude that there was no diel vertical migration for any of these chaetognaths. The three Eukrohnia species, S. macrocepha1a, and $\underline{\text { S. maxima }}$ were probably present below the maximum depth of sampling in the SW and/or NSS as indicated from the vertical distibutions in Figure 4.2 and from published reports, so the median depths calculated for these species did not represent the true population median depths. However, shifts in their 
vertical distributions over a diel cycle should still have been detectable from this analysis. If these species were migrating upward at night from below $1000 \mathrm{~m}$, they should have been more abundant in the night samples. Day/night comparisons of total abundance in the upper $1000 \mathrm{~m}$ for these species showed no significant differences (Table 3.1), thus supporting the results of the day/night median depth comparisons.

Diel vertical migrations may occur only under certain environmental conditions and the inability to detect significant day/night changes in median depth here may reflect the variability of this behavior. Each day/night tow pair was examined for a greater than expected number of species with shallower night than day median depths. Only one day/night pair (MOC 1-60,61) was found to be significant $(p<0.05$, Wilcoxon signed-rank test). Nine of the twelve species in this pair of tows had night median depths shallower than day, and the other three species showed no difference in median depth. One of sixteen tows showing significance at the 0.05 level is not beyond expectation $(p=0.56)$, but it is interesting to note that at this station the water column was isothermal $\left(\sim 18^{\circ} \mathrm{C}\right)$ to approximately $700 \mathrm{~m}$ (Figure 4.1). If diel vertical migration was actually occurring at this station, this argues against McLaren's (1963, 1974) hypothesis that a demographic advantage accrued by migrating across a temperature gradient is the principal adaptive value of this behavior. Pearre (1979) discussed many of the problems associated with using vertically stratified net hauls and resultant mean or median depths to detect diel vertical migration. The detection of this behavior depends upon the cohesiveness and synchrony of a species' behavior. In other words, all individuals must migrate the same distance and they must 
Table 4.3. Day/night differences in the vertical distribution of chaetognaths. 


$\begin{array}{cccc} & \text { Number of day/ } & \text { Mean (day - night) } & \text { Day= } \\ \text { Species } & \text { night pairs } & \text { median depth(m) } & \text { Night* }\end{array}$

Bathypelagic species

E. bathypelagica

E. fowleri 10

$6-9$

NS

E. hamata 10

-9 NS

S. macrocephala 12

12 NS

S. maxima

8

$-10$

NS

NS

Mesopelagic species

K. subtilis

S. decipiens

S. 1yra

S. planctonis

Epipelagic species
10

13

11

11
25

$-2$

$-3$

7
NS

NS

NS

NS
K. pacifica

P. draco

S. bipunctata

S. enflata

S. hexaptera

S. minima

S. serratodentata 13

S. tasmanica

* Wilcoxon signed-rank test

$\begin{aligned} 15 & \text { NS } \\ 4 & \text { NS } \\ 14 & \text { NS } \\ -1 & \text { NS } \\ 3 & \text { NS } \\ 2 & \text { NS } \\ 5 & \text { NS } \\ 33 & \text { p }<0.05\end{aligned}$


migrate together if day/night differences in median depth are to reflect the behavior of individuals. If only some members of the population migrate, the resultant day/night difference in median depth will depend upon the proportion of the population migrating, and this method will always underestimate the extent of the migration of the migrating individuals. Differences in the migratory behavior of sexual maturity stages have been observed for S. elegans (Russel1, 1931; Pearre, 1973). Because older stages are usually less abundant, migratory behavior of these older stages might not be detectable from changes in total population median depth. If individuals migrate asynchronously, temporal variations in median depth will represent some sort of average vertical movement vector. Pearre (1979) suggested that asynchronously migrating populations might produce bimodal vertical distributions (such as that found for S. planctonis from Figure 4.2) regardless of the time of sampling. Such bimodal distributions were rare in these data.

Diel vertical migrations can only be detected from these data if individuals migrated from one depth interval to another, so vertical migrations within the upper $100 \mathrm{~m}$ were not detectable. Because the epipelagic species were primarily present in the upper $100 \mathrm{~m}$, their vertical migrations were not detectable from these data.

Ontogenetic migrations

Length data for seven meso- and bathypelagic species were examined for ontogenetic migrations by dividing the total size range observed for each species into four or five equal length classes and calculating the 
number/1000 $\mathrm{m}^{3}$ for each length class in each of the eight depth intervals sampled for several tows. These length classes were arbitrarily selected for ease in the calculation of the number/1000 $\mathrm{m}^{3}$. Ontogenetic migrations were examined from five tows for S. 1yra, four for $\underline{\mathrm{S}}$. hexaptera, two for E. hamata, and one each for K. subtilis, s. decipiens, S. macrocephala and S. maxima.

Figure 4.3 shows typical patterns of the vertical distribution of the length classes for these species. The larger length classes were usually deeper for all these species and the depth range occupied by each length class was smaller than that of the entire population. An indication of the extent of these migrations is given by the difference in median depths of the shallowest and deepest length classes (Table 4.3).

S. 1yra showed the typical pattern of ontogenetic migration with progressively larger sized individuals at progressively greater depths (Figure 4.3). The ranking of the size classes from smallest to largest corresponded exactly with the ranking of median depth from shallowest to deepest in all five tows for S. lyra. E. hamata displayed the same correspondence of length and depth in both tows from which it was measured and $\underline{S}$. decipiens and $S$. macrocephala also showed the same pattern in single tows. For K. subtilis the second smallest length class was found at a slightly shallower depth than the smallest length class.

For $S$. hexaptera, the second smallest length class was found to be shallowest in all four tows. S. maxima showed an interesting pattern of progressive shoaling in the first three length classes and then deepening of the two largest length classes. Any general theory of the adaptive value of ontogenetic migrations must take into account the differences 


\section{Figure 4.3. Ontogenetic migrations of seven chaetognaths. Arrows indicate median depths for each length class.}




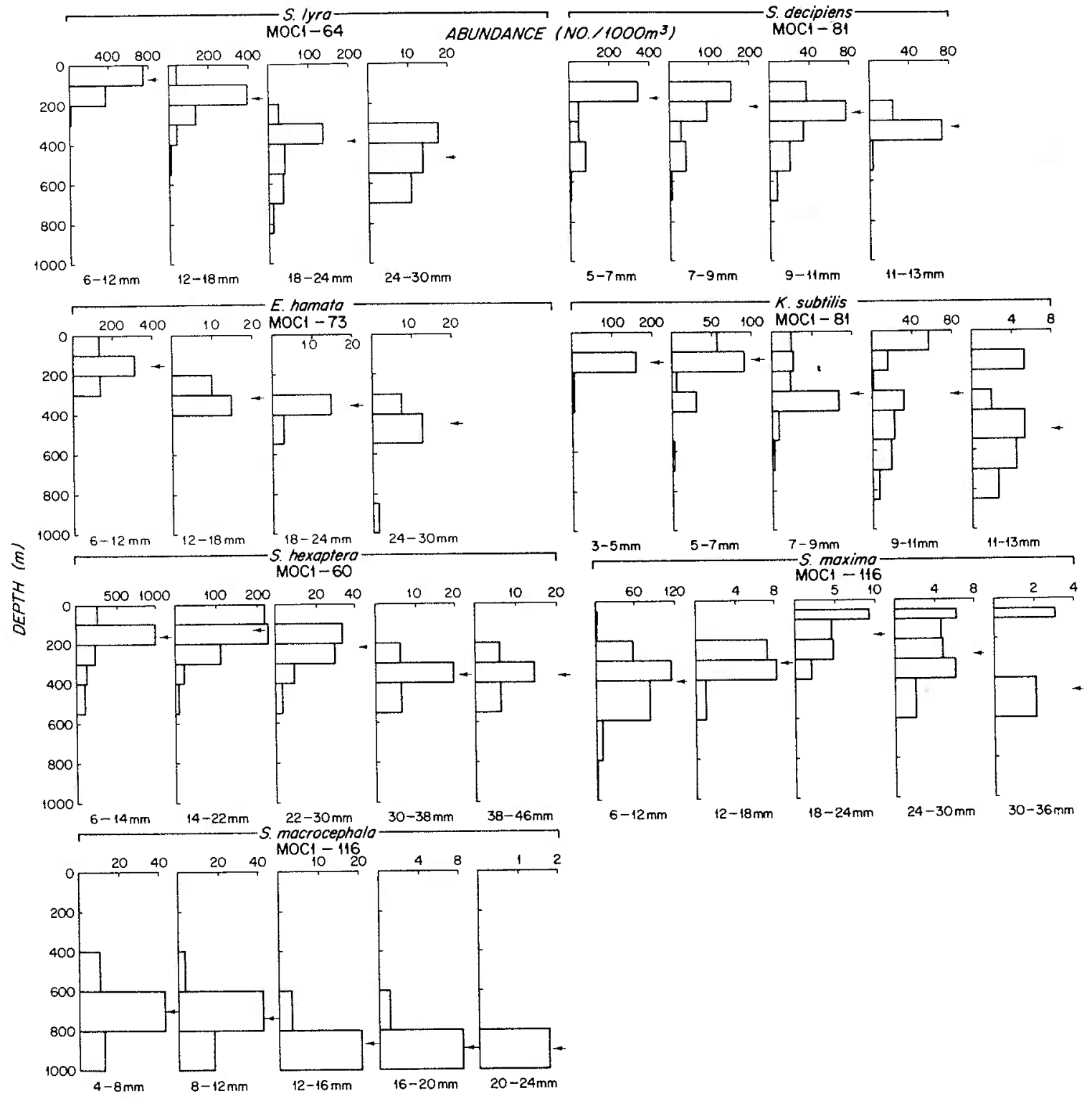


Table 4.4. Extent of the ontogenetic migrations of six chaetognaths. 


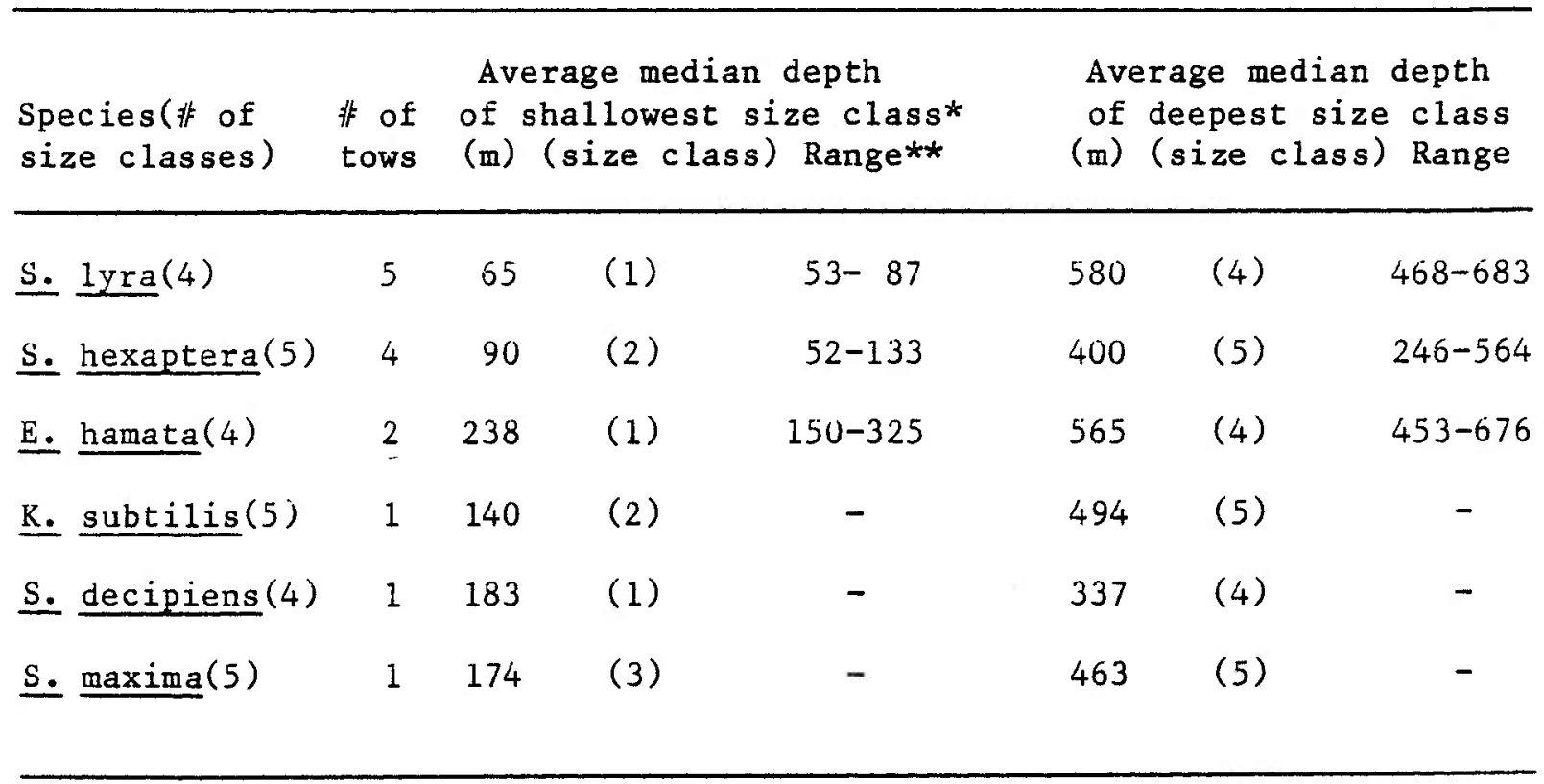

*For size classes, 1 = smallest and 5 = largest, and corresponding length ranges for each species are given in Figure 4.3 .

**This is the range of the median depths for the indicated size class. 
observed in the behavior of these two species.

As discussed earlier, these ontogenetic migrations have an important implication when comparing vertical distributions from different times and places in the ocean. Observed differences in vertical distribution may be a function of differences in population size frequency distribution. Extreme changes in size frequency distribution may lead to changes in population vertical distribution of the order of several hundred meters for some of these species. As an example, suppose we compare the vertical distributions of two populations of S. Iyra, one composed entirely of 6-12 $\mathrm{mm}$ individuals and the other composed of $24-30 \mathrm{~mm}$ individuals. As seen from Table 4.4, we would observe a difference in median depth of over 500 $m$, and in the absence of size frequency data and a knowledge of the ontogenetic migration of this species, we would assume that the difference in median depths was due to behavioral responses of individuals to environmental differences between stations. Although such extreme differences in size frequency distribution are probably uncommon in nature, differences associated with seasonal reproductive patterns or between populations in different water masses may have substantial effects on the observed vertical distributions.

Correlation of vertical distributions with environmental conditions

Because so little is known of the mechanisms by which chaetognaths select their vertical distributions, testing of simple hypotheses appeared to be the best way to begin. The hypotheses to be tested were that these chaetognaths were orienting their vertical distributions to particular 
temperatures or light levels. It was assumed that all members of a population responded in a similar manner to environmental conditions and that median depth differences between stations reflected the responses of individuals to differing temperature or light levels between those stations. Median depth differences have been used in previous studies of the vertical distribution responses of zooplankton populations to differing temperature and light conditions (Moore et al., 1953, Owre, 1960)

Because of the lack of the necessary vertical resolution within the upper $200 \mathrm{~m}$, it was not possible to explore fully the relationships of the epipelagic species' vertical distributions with the physical structure of the water column. A limited analysis, however, of the relationships between median depth and temperature, light, and depth of the mixed layer for several of the epipelagic species was performed. Correlations were found to be inappropriate for these data because of the lack of vertical resolution in the upper $200 \mathrm{~m}$, so a graphical analysis was undertaken.

Because of the depth intervals sampled, the median depth of the epipelagic species reflected the fraction of the population found below $100 \mathrm{~m}$, so temperature and light at $100 \mathrm{~m}$ were considered to be more representative of conditions which altered the median depths than conditions at shallower depths. Initial plots of median depth versus temperature at $100 \mathrm{~m}, 1$ ight at $100 \mathrm{~m}$, and mixed layer depth for $\underline{\mathrm{k}}$. pacifica, P. draco, S. bipunctata, S. enflata, s. minima, S.

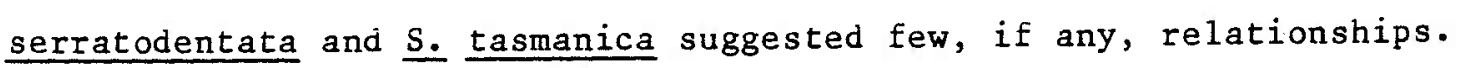
Removal of species from tows in which fewer than ten individuals were identified and removal of four tows (MOC1- 18, 19, 116, 117) for which 
surface salinity was less than 34.8 ppt revealed some interesting patterns for several species. Apparently, conditions which reduced populations to fewer than ten individuals also considerably altered the vertical distributions of these species. The presence of low salinity water at the stations listed above suggests transport of coastal water over the underlying slope Water, considerably altering the vertical distributions of these species.

No species showed any relationship of median depth with light at $100 \mathrm{~m}$. Mixed layer depth did appear to be related to median depth for P. draco,

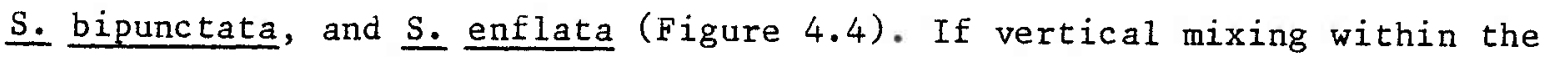
mixed layer is great enough, we might expect to see a uniform distribution of a population within the mixed layer. For MOC 1-54, the depth of the mixed layer was $160 \mathrm{~m}$ and the upper $200 \mathrm{~m}$ was sampled by three nets (0-50 $\mathrm{m}, 50-100 \mathrm{~m}$, and $100-200 \mathrm{~m}$ ). Assuming that no individuals occurred below $160 \mathrm{~m}$, I calculated the expected number of individuals for eight species in each of the three samples. Chi-square goodness of fit tests indicated that for six species, K. pacifica, p. draco, S. bipunctata, S. enflata, S. $\underline{\text { hexaptera }}$ and $\underline{S}$. lyra, the vertical distributions within the mixed layer were not significantly different from a uniform distribution. Only $\underline{S}$.

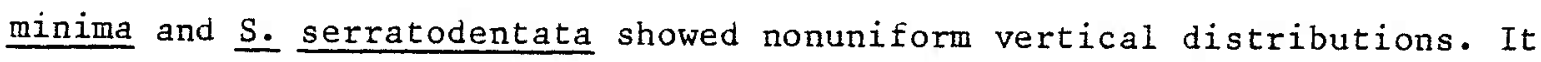
is interesting to note that these two species also showed no consistent relationship of median depth with depth of the mixed layer. With the exception of $\underline{S}$. hexaptera and $\underline{S}$ tasmanica, the epipelagic species appeared to be limited primarily to the surface mixed layer. They were occasionally found below the seasonal thermocline, but these instances could not be related to any of the measured variables. It is presumed that 
Figure 4.4. Relationships of median depths for four epipelagic species with depth of the mixed layer and temperature at 100 m. 


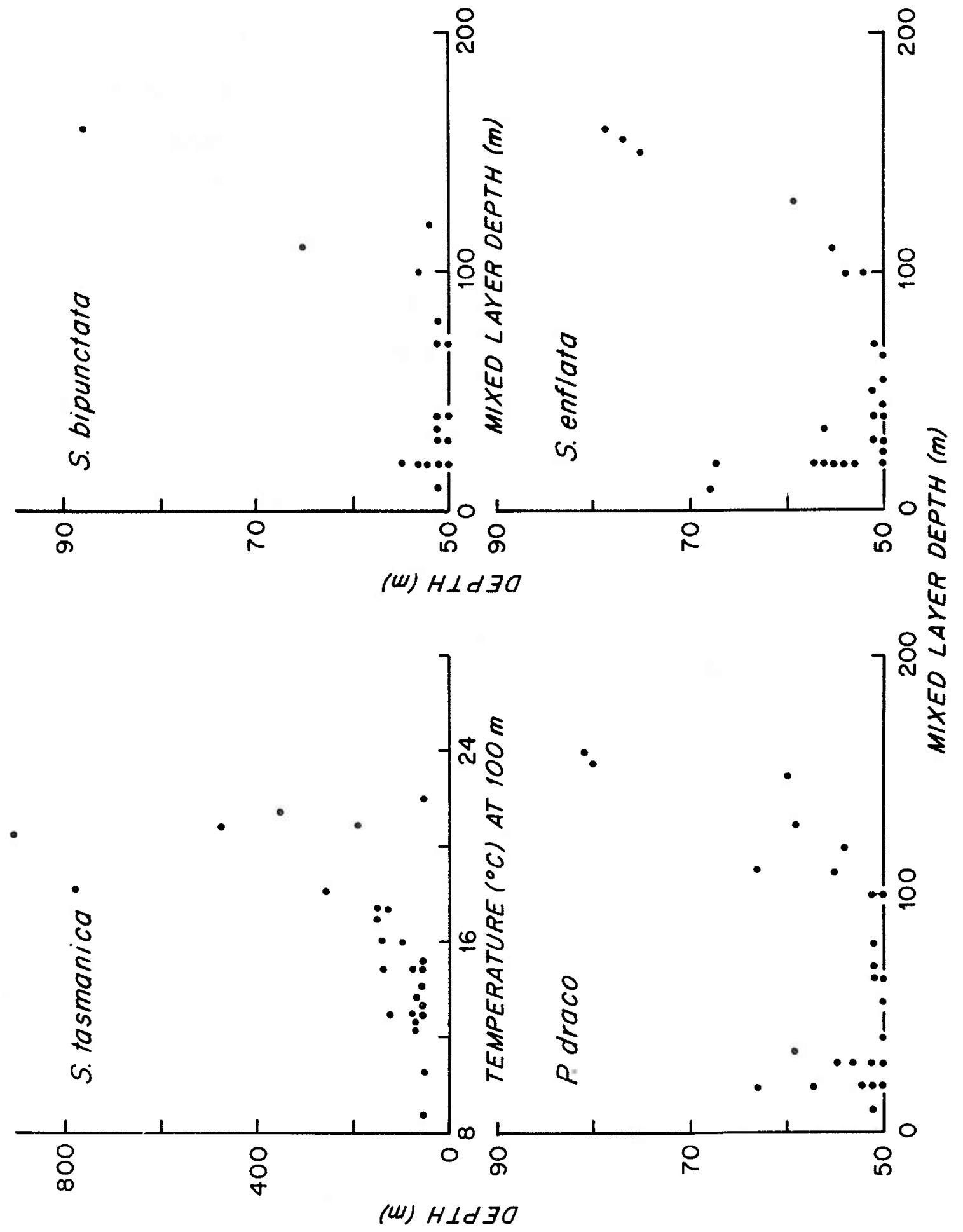


low temperatures limit their penetration into deeper waters, but better vertical resolution within the upper $200 \mathrm{~m}$ is needed to test this hypothesis.

The only epipelagic species to show a relationship with temperature at $100 \mathrm{~m}$ was S. tasmanica (Figure 4.4). Only the tows taken in the low salinity surface water were removed from this plot. The Spearman rank correlation coefficient for these data was $0.63(p<0.01)$. Individuals were rarely observed in the NSS, but when present were most often found at great depth. Personal observations made while SCUBA diving in the North Atlantic suggested that $\underline{S}$ tasmanica was found only in and below the depths of the seasonal thermocline, but again the coarse vertical resolution of these tows did not allow the testing of this hypothesis.

A more detailed analysis of the relationship between median depth and temperature and light was carried out for the following species- E. hamata, K. subtilis, S. decipiens, S. hexaptera, S. lyra, S. macrocephala, and $\mathrm{S}$. maxima. The hypothesis to be tested is as follows:

\footnotetext{
Each species lives at its own specific temperature, light level, or combination thereof.
}

There is obviously variability in the depths occupied by individuals within plankton populations, so it was assumed that the median depth for each tow represented the optimal temperature and/or 1 ight conditions. This hypothesis may be tested by a multiple regression of median depth on the depth of the appropriate isotherm and isolume for each species. The large ontogenetic migrations and the deeper vertical distributions of $\underline{\mathrm{K}}$. 
subtilis and S. lyra in the SW, however, suggested an alternative hypothesis to explain differences in vertical distribution:

Differences in median depth between stations are due to ontogenetic migrations coupled with differences in size frequency distributions.

Rather than perform a complete multiple regression analysis for each species, I used a simpler correlation analysis as a preliminary test of these two hypotheses.

A brief discussion of the correlation procedure is necessary. Median depths were calculated for each of these species from a total of 52 MOCNESS tows taken in the SW (18), NSS (18), Gulf Stream (2), and Gulf Stream cold core rings (14). Usually fewer than 52 tows were available for any species for reasons discussed in Chapter 2. Direct correlations of median depth with temperature or light from that depth were not useful as discussed earlier. Temperatures for correlation were determined from a specified depth for each species. That depth was determined as the average median depth, rounded to the nearest $25 \mathrm{~m}$, in the hydrographic region where the species in question was most abundant. Hydrographic region of greatest abundance (Table 5.1) and depth used for E. hamata was SW and 350 $\mathrm{m}$, for $\mathrm{K}$. subtilis was $\mathrm{NSS}$ and $275 \mathrm{~m}$, for $\mathrm{S}$. decipiens was NSS and $275 \mathrm{~m}$, for S. hexaptera was NSS and $100 \mathrm{~m}$, for $\mathrm{S}$. Iyra was NSS and $125 \mathrm{~m}$, for $\mathrm{S}$. macrocephala was SW and $700 \mathrm{~m}$, and for $\mathrm{S}$. maxima was $\mathrm{SW}$ and $450 \mathrm{~m}$.

Light was calculated as discussed earlier, and the $\log _{10}$ (noon light) value at $100 \mathrm{~m}$ was used in all correlations. Because chlorophyll a values were generally insignificant below $100 \mathrm{~m}$, calculated $\log _{10}$ (noon light) 
values below $100 \mathrm{~m}$ differed by an approximately constant value between any two tows. Thus, $\log _{10}$ (noon light) at $100 \mathrm{~m}$ adequately expressed the differences between tows at any depth greater than $100 \mathrm{~m}$. Noon light levels were chosen because these chaetognaths showed no significant diel vertical migration. It was assumed that if they were orienting their depths of occurrence by light, they may have been using either a maximum or average daily light level. Maximum light levels occur at noon (discounting the effects of variable cloudiness), and the $\log _{10}$ (noon light) levels were highly correlated ( $r=0.99$, product-moment correlation coefficient) with the $\log _{10}$ average daily light calculated from equations presented by Reed (1977).

As discussed earlier, observed spatial and temporal differences in vertical distribution may be a function of changes in population size frequency distribution as we 11 as changes in environmental conditions. Size trequency distributions were available from all these tows only for S. Iyra. Its average individual size was calculated for each tow and was also correlated with median depth.

A discussion of the expected outcomes will make it easier to interpret the results of the correlation analysis. If a species' vertical distribution follows an isotherm, then the median depth should be positively correlated with temperature. The same holds true for light. If shifts in median depth are due to changes in size frequency distribution, a significantly positive correlation should be seen for median depth and average individual size.

I will begin with a discussion of the results for $\underline{S}$. lyra before proceeding to the other species. The correlation matrix for $\underline{S}$. lyra is 
presented in Table 4.5. The significant negative correlation of median depth and temperature was in opposition to the hypothesis that median depth is associated with a particular temperature for $\underline{S}$. lyra. This was in accord with the finding that this species was significantly deeper in the SW (Figure 4.2 and Table 4.2). The significant positive correlation of median depth with average individual size supported the hypothesis that the observed vertical distributions were largely a function of differences in population size structure. Average individual size was larger and population size was smaller in the SW for S. lyra (both, $p<0.01$, Mann-Whitney U test). Either a small breeding population of $\underline{\text { S. }}$ lyra was maintained in the SW or it was transported into the SW across the Gulf Stream from the south by horizontal mixing processes such as Gulf Stream warm core rings. It is clear that median population depth was an inadequate measure for determining the relationships of vertical distribution of individuals with temperature and light.

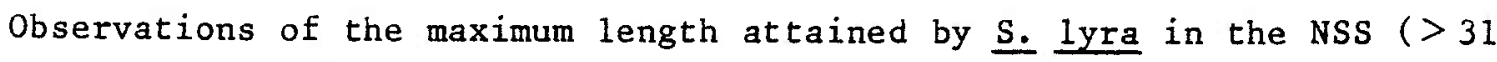
$\mathrm{mm})$ and the $\mathrm{SW}(>41 \mathrm{~mm})$ suggested that individuals were living at colder temperatures in the SW. Maximum body size has been found to be inversely correlated with temperature for many marine invertebrates (Kinne, 1970, pp. 511-512). McLaren (1966) reported greater maximum length with decreasing temperature for $\underline{S}$. elegans from field collections, and Reeve and Walter (1972) reported an inverse relationship for length at maturity and temperature for $\underline{S}$. hispida from laboratory experiments. This observation supported the second hypothesis and was in opposition to the hypothesis that this species follows an isotherm.

There is obviously a need to assess the effects of ontogenetic 
Table 4.5. Correlations of median depth with environmental variables for $\underline{S}$. 1yra. The number of tows upon which the correlation coefficient was based is given in parentheses $(*=p<0.05, * *=p<0.01)$ 


\begin{tabular}{|c|c|c|c|c|}
\hline & $\mathbf{T}$ & $\mathrm{L}$ & $\mathbf{S}$ & $\mathrm{p}$ \\
\hline Median depth (D) & $-.572(48) * *$ & $.225(41)$ & $.794(48) * *$ & $-.489(48) * *$ \\
\hline Temperature ( $\mathrm{T}$ ) & 1 & $.313(45) *$ & $-.856(48) * \star$ & $.509(48) * *$ \\
\hline Light (L) & & 1 & $.023(41)$ & $-.144(41)$ \\
\hline $\begin{array}{l}\text { Average individual } \\
\text { size (S) }\end{array}$ & & & 1 & $-.575(48) * *$ \\
\hline $\begin{array}{l}\text { Population } \\
\text { abundance (P) }\end{array}$ & & & & 1 \\
\hline
\end{tabular}


migrations and population size structure changes on the vertical distributions of the remaining species, but size frequency distributions were not available from these tows. The significant correlation of average individual size and population abundance for $\underline{S}$. lyra suggested that population abundance was an adequate indicator of average individual size. Median depth and population abundance were significantly negatively correlated, and this correlation was due to the relationship of average individual size and population abundance as indicated by the partial correlation coefficient of $-0.065(\mathrm{p}<0.05)$ for median depth and population size after removal of average individual size effects.

Correlation coefficients of median depth with temperature, light, and population size are presented for the remaining six species in Table 4.6, and $\underline{S}$. lyra was included for comparative purposes.

The correlation coefficients for $\underline{K}$. subtilis were quite similar to those for S. Iyra, suggesting that population size structure changes were also responsible for many of the observed differences in its median depth.

Neither $\underline{S}$. decipiens nor $\underline{S}$. hexaptera showed any significant correlation and neither showed significant differences in median depth between NSS and SW (Table 4.2). Both species were ontogenetic migrators, so the lack of a significant correlation of median depth and population abundance may reflect the inadequacy of population abundance as an indicator of population size frequency distribution.

All three bathypelagic species showed significant positive correlations with temperature in accord with the preliminary analysis from Figure 4.2 and Table 4.2. S. maxima was the only species with a significant correlation with light, but this may have been due to chance. The 
Table 4.6. Correlations of median depth with temperature (T), light (L) and population abundance (P) for seven chaetognath species. The number of tows upon which the correlation coefficient was based is given in parentheses $(*=p<$ $0.05, * *=p(0.01)$ 


\begin{tabular}{lll}
$\mathrm{T}$ & $\mathrm{L}$ & $\mathrm{P}$ \\
\hline
\end{tabular}

S. Iyra

$-.572(48) * *$

$.225(41)$

$-.489(48) * *$

K. subtilis

$-.535(47) * *$

$.022(40)$

$-.529(47) * *$

S. decipiens

$-.093(50)$

$-.057(43)$

$.098(50)$

S. hexaptera

$-.133(48)$

$.199(41)$

$-.021(46)$

s. maxima

$.739(35) * *$

$.369(28) *$

$-.280(35)$

S. macrocephala

$$
.936(34) * *
$$

$.135(27)$

$-.777(34) * *$

E. hamata

$.952(38) * *$

$.126(31)$

$-.773(38) * *$ 
probability of obtaining one or more significant correlations at the 0.05 level in seven tests of median depth with light is 0.30 . That E. hamata and $\underline{S}$. macrocephala median depths were significantly negatively correlated with population abundance again suggested that population size structure changes may be responsible for the observed differences in median depth. Although population abundances for the bathypelagic species were underestimates, especially in the NSS, I believe that the same trends in population abundance would be found if the entire populations were sampled. The lack of a significant negative correlation with population abundance for $\underline{\mathrm{S}}$ maxima may be due to its 'abnormal' ontogenetic migration (Figure 4.3).

An examination of the vertical distribution of these species in cold core rings serves to better illustrate the results of this analysis. The data from the cold core rings do not constitute an independent test of these hypotheses, however, as these data were also used in the correlation analysis.

Ring D, a cold core ring formed in February 1975, was sampled with the MOCNESS in August and November of 1975. Figure 4.5 shows the changes in isotherm depth with time and concomitant shifts in median depth for nine species. Only $\underline{\mathrm{S}}$ macrocephala and $\underline{\mathrm{S}}$. maxima were deeper in November than in August, and these differences were not significant. Five species showed significantly different median depths, and in each case the median depth was shallower in November. These results were inconsistent with the hypothesis that median depths were associated with specific isotherms. Average individual size for $\underline{S}$. 1yra was $22.0 \mathrm{~mm}$ in August and $11.8 \mathrm{~mm}$ in November, again in accord with the hypothesis that population size 
Figure 4.5. Relationships of isotherm depth with median depths for nine chaetognaths in Ring $D$. Asterisks denote significant differences in median depth $(*=p<0.05$, $\left.\star * x=\mathrm{p}<0.01 . * * *_{\mathrm{p}}=<0.001\right)$. 


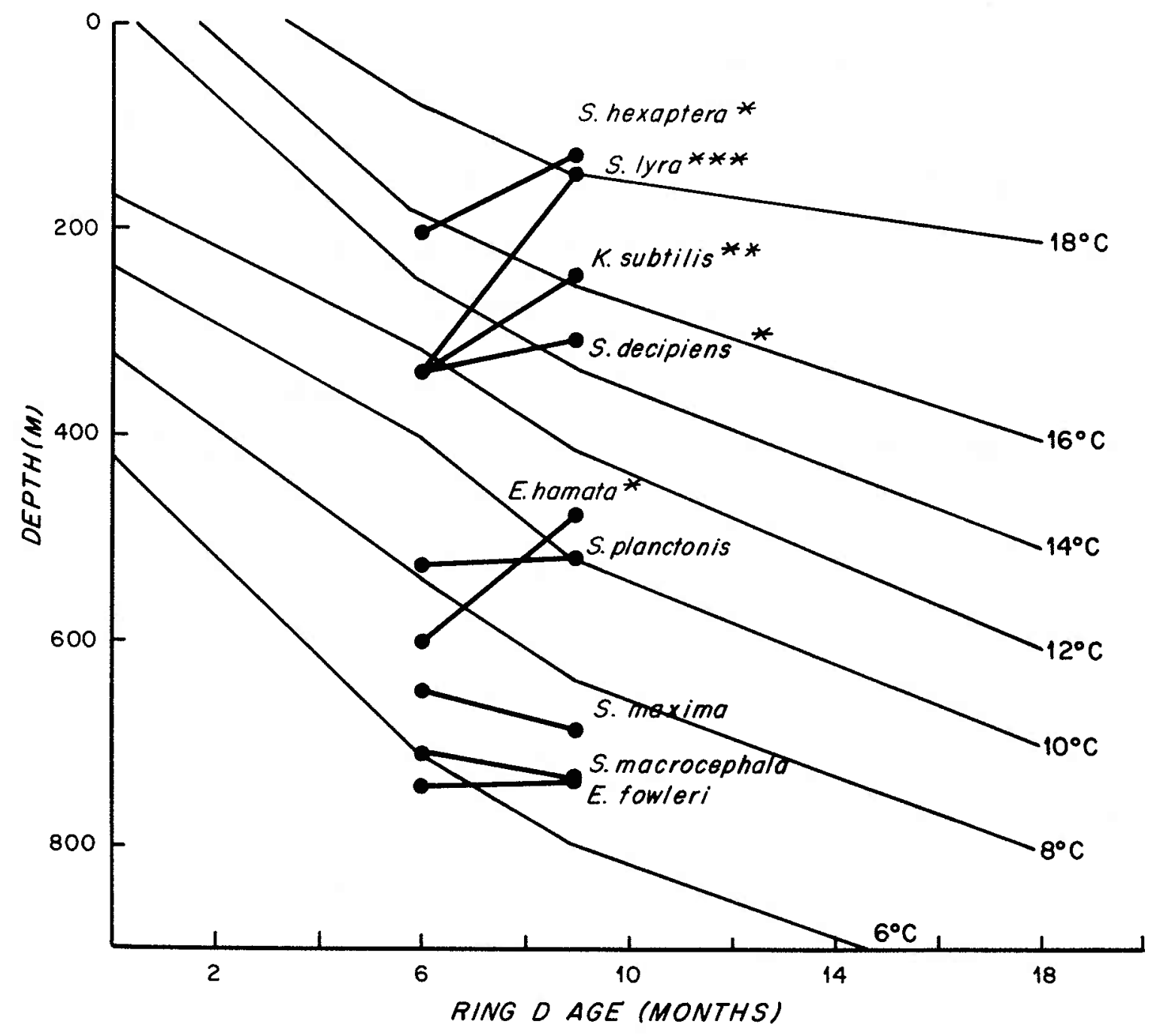


structure differences were responsible for the observed differences in median depth.

During the Endeavor 11 cruise of August 1977, a series of MOCNESS tows was collected at varying distances from the center of Ring Bob. Median depths for ten species have been plotted as a function of distance from the ring center in Figure 4.6. MOCNESS tows $77,78,84,87$, and 83 represent ring center stations, tows $85,90,86$, and 89 represent ring fringe stations, and tows $82,81,80$, and 79 represent NSS stations. The depths of the isotherms in Figure 4.6 show the expected relationship of median depth with distance from the ring center if these organisms were behaviorally orienting their depths to isotherms. E. fowleri, E. hamata, S. macrocephala, S. maxima, S. planctonis, and S. tasmanica were found progressively deeper as distance from the ring center increased, supporting the hypothesis of temperature regulation of depth. None of the species can be said to be closely following a particular isotherm, but this may be due in part to inaccurate median depths for the bathypelagic species (some unknown fraction of their populations occurred below 1000 m). Although there is no size frequency data available to test the hypothesis, if differences in population abundances were correlated with average individual size as discussed for $\underline{S}$. lyra earlier, then the patterns for these species could be due to size frequency distribution differences. Abundances for each of these species decreased as distance from ring center increased.

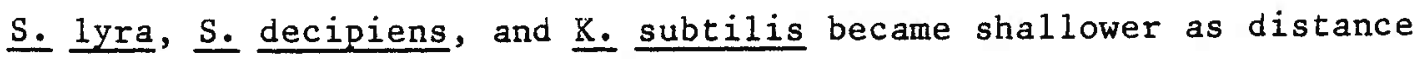
from ring center increased, in accord with the earlier observations of the behavior of these species. The median depths for these species were 
Figure 4.6. Isotherm depths and median depths of ten chaetognaths plotted as a function of distance from the center of Ring Bob. 


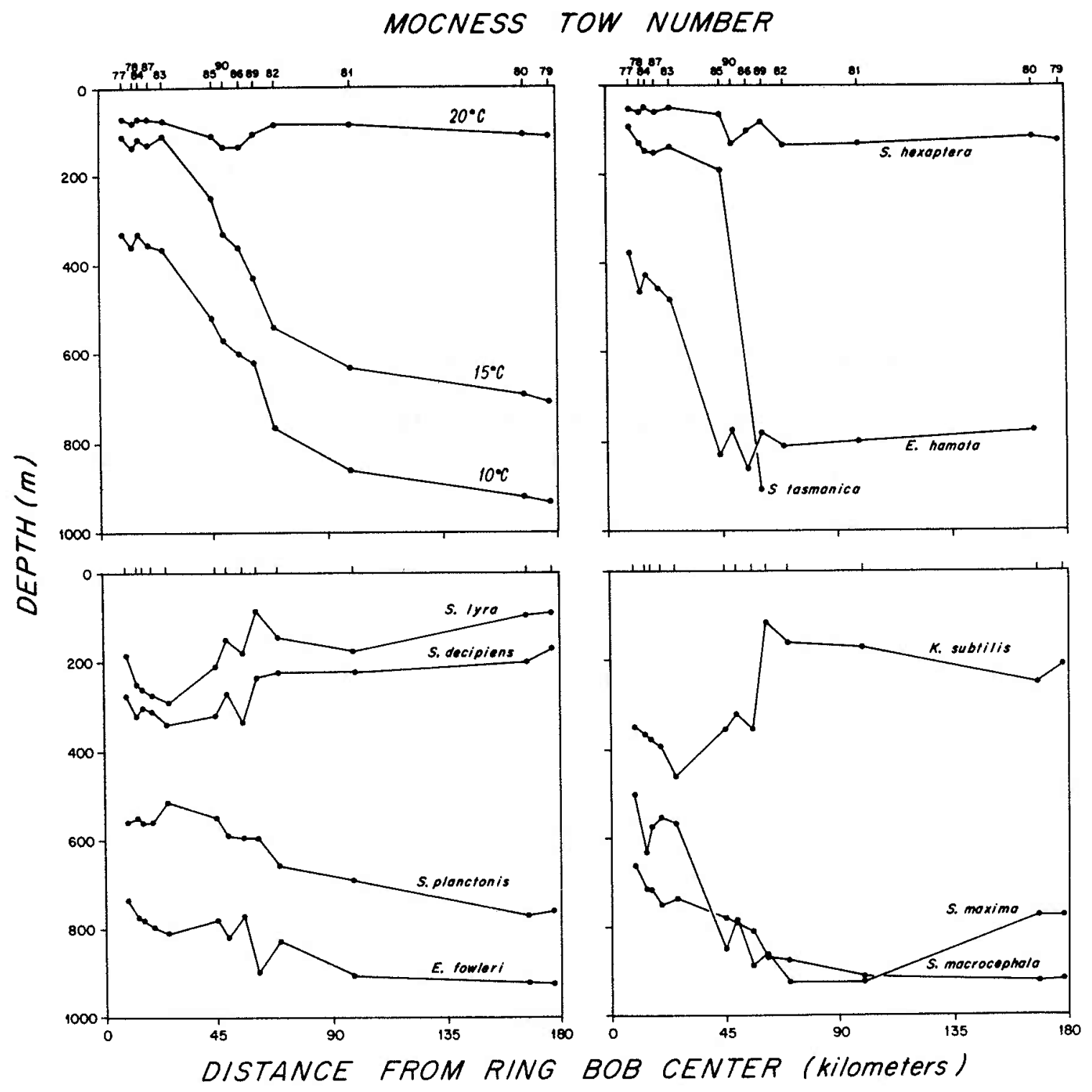


obviously not following isotherms or isolumes. The Kenda11 rank correlation coefficient for $\underline{S}$. lyra median depth and average individual size for these tows was $0.84(p<0.01)$, again supporting the hypothesis that size frequency distribution changes were responsible for the shoaling of these populations away from ring center.

The S. hexaptera population became slightly deeper away from the ring center and was maintained in the vicinity of the $20^{\circ} \mathrm{C}$ isotherm. Correspondence of median depth with this isotherm was far from exact. Population abundances increased in the NSS, so it was expected that $\mathrm{S}$. hexaptera should be shallower in the NSS. This may again reflect the inadequacy of population abundance as a measure average individual size for this species, as was found in the correlation analysis.

In summary, what can now be said about the regulation of the vertical distributions of chaetognaths in the western North Atlantic Ocean? For the epipelagic species, temperature is the most likely factor limiting their penetration into deeper waters. Much better vertical resolution is needed to fully assess the regulation of their vertical distributions.

For the mesopelagic species, K. subtilis, S. decipiens, S. Iyra, and $\underline{\text { S. }}$ hexaptera (considered here to be functionally related more to the mesopelagic than epipelagic species), the hypothesis that temperature and/or light were responsible for the observed differences in median depth can be discarded. The hypothesis that ontogenetic migrations coupled with size frequency distribution changes appears more appropriate. An analysis of the relationship of the median depths of size or sexual maturity classes with temperature, light, and depth is needed. Such an analysis is currently underway for $\underline{S}$. Iyra and will be reported in a subsequent paper. 
It has not been possible to distinguish between the hypotheses of temperature control and population size structure changes for the bathypelagic species. Analysis of the size frequency distributions of these species and sampling below $1000 \mathrm{~m}$ are necessary to distinguish between these two hypotheses.

It is probably unwise to discount the role of light in regulating the vertical distributions of any of these species. While we can say that they are probably not responding to diel fluctuations in light level as indicated by the lack of diel vertical migrations, they may be averaging light over some longer time interval.

Discussion

Sampling problems and vertical distributions

Although zooplankton ecologists are aware of the many problems associated with net sampling, we often ignore these problems in the interpretation of vertical distribution patterns. The biases and imprecision of net sampling can result in observed vertical distributions which are quite unrepresentative of the natural patterns. A brief discussion of the effects of some of these problems can aid in the interpretation of spatial and temporal patterns of zooplankton vertical distributions.

Mesh selection often results in the undersampling of younger, smaller individuals. Observed vertical distributions only represent the patterns 
of the size classes caught. If all size classes within a population are distributed similarly, then the observed pattern is little different from the natural pattern. For organisms which migrate ontogenetically, the observed patterns do not represent the vertical distribution of the total population. Many zooplankters are ontogenetic migrators (Vinogradov, 1970), so care must be taken in interpreting the observed patterns, especially when comparing vertical distributions from studies using different mesh sizes.

Avoidance of towed nets may also influence the observed vertical distributions. The patterns for organisms which do not visually detect and avoid nets or migrate ontogenetically should not be affected much by avoidance. Organisms which visually detect and avoid nets may reveal some rather interesting "behaviors". Shallower organisms should be more capable of detecting and avoiding nets during the day than deeper living individuals because of the greater light nearer the surface. At night, the entire population of a visual avoider is equally likely to be sampled by the net. The resulting diel patterns of vertical distribution could be interpreted as diel vertical migration, but a comparison of total abundance in day and night samples will reveal greater night abundances if this is a problem. No day/night differences in abundance were observed for the chaetognaths in this study, but for other zooplankters such as euphausiids (Brinton, 1967), the vertical distibutions observed during the day may be quite biased. In general, larger individuals of a species swim faster than do smaller individuals, so the vertical distribution of an ontogenetic migrator will be biased by the greater avoidance of the larger, deeper individuals. This may be a problem for several of the 
larger chaetognaths in this study.

As was discussed earlier, small scale patchiness may result in observed differences in vertical distribution over small time or space scales on which environmental conditions do not change. This is primarily a problem in the study of diel vertical migrations (Cushing, 1951), and its effects were apparent in some studies of chaetognath diel vertical migration (Moore, 1949; Pearre, 1974). One must be cautious in the interpretation of such time series studies of vertical distribution if there are large variations in total abundance. Use of the largest sized net and longest tow length possible are recommended to reduce the effects of patchiness on observed vertical distributions (Wiebe, 1971, 1972). Subsampling variability may also produce observed differences in vertical distribution.

The nature of the sampling procedure may also affect the vertical distribution patterns observed. Banse (1964) discussed problems of previous studies, and he argued that horizontal hauls at specified depths should be employed rather than vertical or oblique tows, which obscure the relationships between the vertical distributions and environmental conditions. Such a procedure does not, however, sample the entire population under study. Oblique hauls in adjacent depth intervals which cover the entire vertical range occupied by a species are recommended here. The size of the depth intervals sampled can be varied to obtain detailed resolution around strong hydrographic gradients such as the thermocline and still provide total coverage of the water column. The recent development of multiple opening/closing nets greatly facilitates such a sampling procedure. Diel vertical migration has occasionally been studied by time series sampling at one or two depths (Welsh et al., 1937; 
Roe, 1974). The data from these studies is ambiguous because of the problem of horizontal patchiness and provides no information on the extent of the migrations. This sampling procedure is not to be recommended. As is evident from the results of this study, there are problems associated with the use of mean or median depth as a measure of vertical distribution. These problems are associated with differences in behavior between individuals. If these behavioral differences can be correlated with identifiable characteristics such as size or developmental stage, then median depths calculated separately for each group should be useful for comparisons between tows. This statistic, however, provides no information about the spread or shape of the vertical distributions. Interpercentile depth ranges, such as the difference between the $75 \%$ and $25 \%$ depth levels or the 90 and $10 \%$ depth levels, have been used for such purposes by a number of authors (Moore, 1949; Owre, 1960; Kolosova, 1972).

Vertical distributions and migrations

The average vertical distributions reported here correspond well with most reports from other regions (Alvarino, 1965, 1967; Fagetti, 1972; Miche1 and Foyo, 1976; Nair, 1978; Stone, 1969; Thomson, 1947). Perhaps the main reason for studying vertical distributions in previously unstudied regions is for comparative purposes. Comparisons of vertical distributions from regions with different vertical structures of temperature, salinity, etc. can be used to elucidate the factors regulating the observed patterns. The ontogenetic migrations reported here and by others complicate comparisons of vertical distribution between 
studies, and with this in mind, I will now discuss several reported vertical distributions which differ substantially from the patterns reported here. Because most previous studies do not present physical data, mesh size of the net, or population size frequency distributions it is impossible to determine exactly why the patterns differ from those reported here.

Alvarino $(1964,1965)$ reported K. subtilis to be an epipelagic species in the world's oceans and Fagetti (1972) also reported this species as epipelagic in the southeastern Pacific Ocean off the coast of Chile. It was found to be primarily mesopelagic here and by other authors (Bieri, 1959; Aurich, 1971; Michel and Foyo, 1976). These differences may be due to population size frequency distribution differences or to mesh selection. When reproduction is occurring or when there are relatively few large individuals, this species will be found primarily within the upper $200 \mathrm{~m}$. Nair (1978) showed some very interesting latitudinal variations in vertical distribution for $K$. subtilis in the Indian Ocean, and it would be interesting to know if these trends were related to behavioral responses to environmental conditions or to population size structure differences. Stone (1969) reported S. bipunctata from the Agulhas Current to be primarily abundant from 100 to $200 \mathrm{~m}$. He reported that this species was not an ontogenetic migrator, so it appears that its deeper vertical distribution may be related to behavioral responses to environmental differences, but no physical data were included for assessment of its responses.

Both Alvarino (1967) and Fagetti (1972) found $\underline{S}$ decipiens to be quite abundant in the upper $100 \mathrm{~m}$ off San Diego, California and Chile, 
respectively. This study and that by Michel and Foyo (1976) showed S. decipiens to be very rare in the upper $100 \mathrm{~m}$ in the western North Atlantic. Alvarino (1967) believed its presence in the upper $100 \mathrm{~m}$ was an indicator of upwelling, as did Stone (1969). Whether or not this involves passive changes in vertical distribution due to upward vertical currents or active orientation to decreased surface temperatures characteristic of upwelling events remains unclear.

Lyons (1970) reported $\underline{S}$. enflata to be most abundant at about $200 \mathrm{~m}$ in winter samples from the Central North Pacific. This was obviously not a response to temperature because winter temperatures were colder than in summer and Lyons found no evidence of ontogenetic migration. Stone (1969), however, reported juveniles higher in the water column than adults for $\mathrm{S}$. enflata in the Agulhas Current. Lyons' sampling scheme in winter and summer were different, so it is not clear that his winter/summer comparisons of vertical distribution were valid. In summer, he sampled six depth intervals obliquely from the surface to $600 \mathrm{~m}$ with $0.505 \mathrm{~mm}$ mesh, and in winter, he sampled five depths with horizontal tows using $0.183 \mathrm{~mm}$ mesh.

Fagetti (1972) found the populations of $\underline{S}$. hexaptera and $\underline{S}$. Iyra almost entirely within the 100 to $200 \mathrm{~m}$ depth interval off Chile. Both species showed ontogenetic migrations in the present study, so the differences may be related to size frequency distribution differences. Nair (1978) found interesting latitudinal variations for these species as well in the Indian Ocean.

S. macrocephala was found in relatively shallow waters (up to $200 \mathrm{~m}$ ) in the southern part of the Indian Ocean by Nair (1978). The temperature 
structure of the water column appeared to be similar to that of the slope water, yet $\underline{\mathrm{S}}$ macrocephala was rarely found above $400 \mathrm{~m}$ in this study. This points to the necessity of providing abundance versus depth profiles rather than upper and lower limits of vertical distribution.

Both Fagetti (1972) and Lyons (1976) showed S. maxima to be very abundant in the upper $200 \mathrm{~m}$. S. maxima was an ontogenetic migrator here, so these differences may be related to population size structure

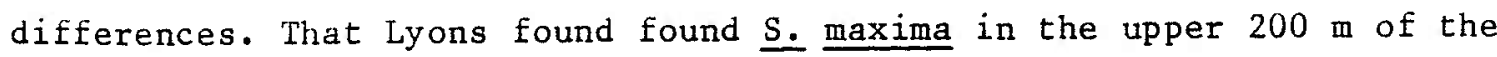
Central North Pacific is quite striking. Neither Bieri (1959) nor Alvarino (1964) reported this species from the upper $500 \mathrm{~m}$ in this region previously. It is possible that Lyons misidentified another species as $\underline{S}$ maxima.

A number of authors have reported $\underline{S}$. minima to be primarily abundant between 100 and $300 \mathrm{~m}$ (Thomson, 1947; Bieri, 1959; Lyons, 1976; Nair, 1978). This species matures at a very small size $(\sim 8 \mathrm{~mm})$ and has been reported to be an ontogenetic migrator (Alvarino, 1964; Stone, 1969). Mesh sizes used in most studies of vertical distribution (including the present one) are probably not adequate for sampling the vertical distribution of this species.

Perhaps the most interesting differences in vertical distribution were reported by Owre (1960) from the Florida Current off Miami. Eleven epiand mesopelagic species showed normal vertical distributions at her NG station, yet only two species, $\underline{K}$ subtilis and $\underline{S}$. decipiens, can be considered as having normal vertical distributions at her SL station. $\underline{\text { P. }}$ draco, K. pacifica, S. enflata, S. hexaptera, and S. serratodentata had median depths of approximately $300 \mathrm{~m}( \pm 25 \mathrm{~m})$, and $\underline{\mathrm{S}}$. 1yra had a median 
depth of $605 \mathrm{~m}$. K. subtilis and S. decipiens were also deeper at the SL station, but the bottom at $350 \mathrm{~m}$ may have cut off the deep members of their populations at the NG station. Owre did not detect any ontogenetic migrations for any of these species. She related the differences in depth at these two stations to temperature and found that the temperature at the median depth of most of the species corresponded very well between the stations. Stepien (1980) recently reported the occurrence of epipelagic chaetognaths and pteropods with meso- and bathypelagic species at approximately $600 \mathrm{~m}$ in the Straits of Florida. These observations were associated with deep southwestward flow in the Florida straits (normal flow is northeastward) and suggested downward transport of shallow oceanic waters during these periods of southwestward flow. Stepien believes that these downwelling events may have been responsible for Owre's anomalous observations of chaetognath vertical distribution. That vertical water transport may alter the vertical distributions of chaetognaths was illustrated by the uniform distributions of six chaetognaths from MOC 1-54 (see Results) and was suggested for other zooplankton by Banse (1964). Further studies are needed in the Straits of Florida to confirm Owre's observations and to assess the causal mechanisms for the vertical distributions.

Large latitudinal variations in vertical distribution have been reported for many bathypelagic chaetognaths (Alvarino, 1964, 1965; Nair, 1978). The observations of deeper vertical distributions in the NSS for the bathypelagic chaetognaths here were consistent with the observations of submergence in regions of warmer water reported by earlier authors. The results of previous studies of the diel vertical migration of 
Table 4.7. Previous studies of the diel vertical migration of chaetognaths found in this study. $0=$ no diel vertical migration, $1=<50$ m difference in day/night median depths, $2=50-100 \mathrm{~m}$ difference, $3=$ greater than $100 \mathrm{~m}$ difference, and ?= migration reported but no estimate of extent was available. 


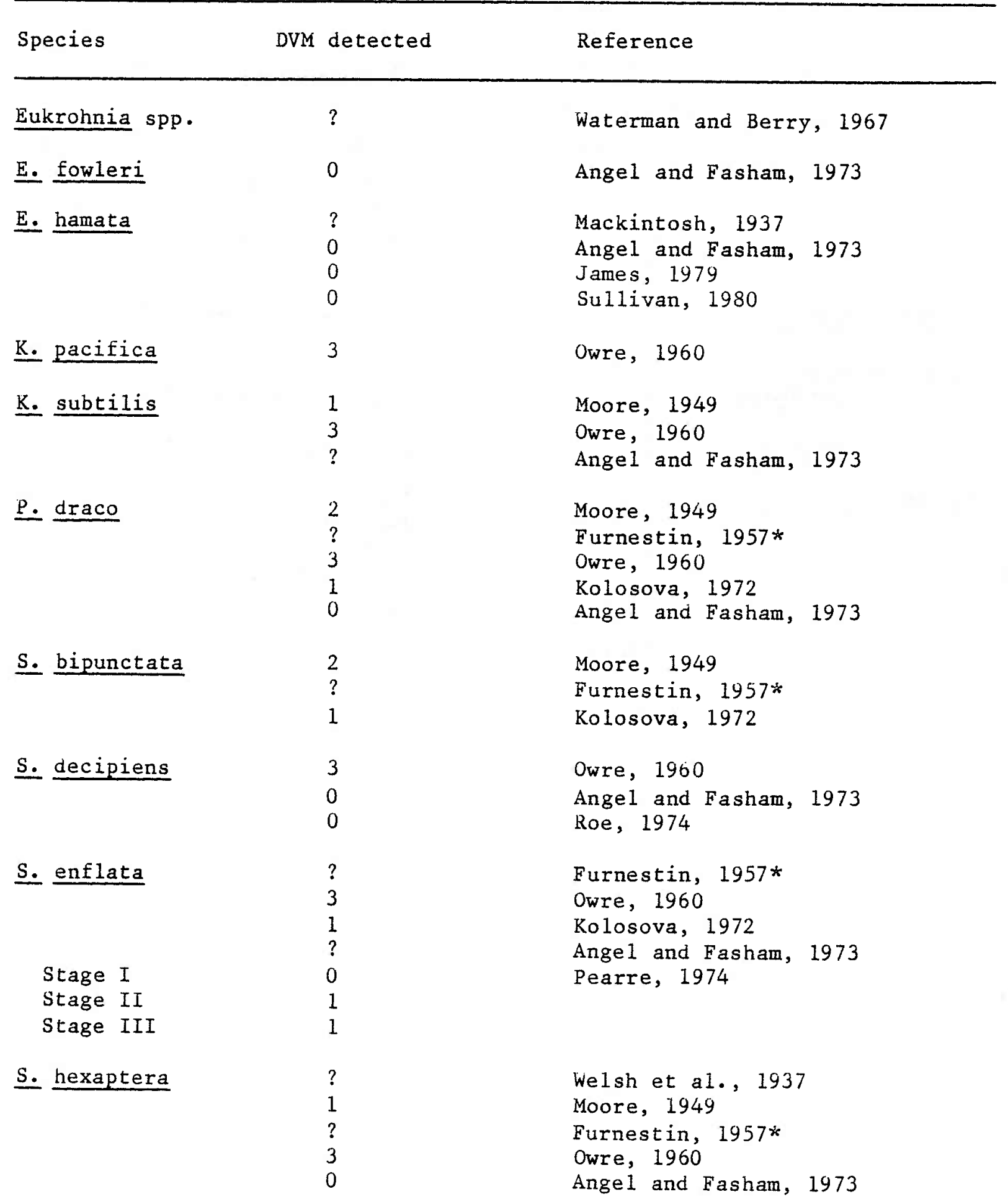


Table 4.7. (cont'd).

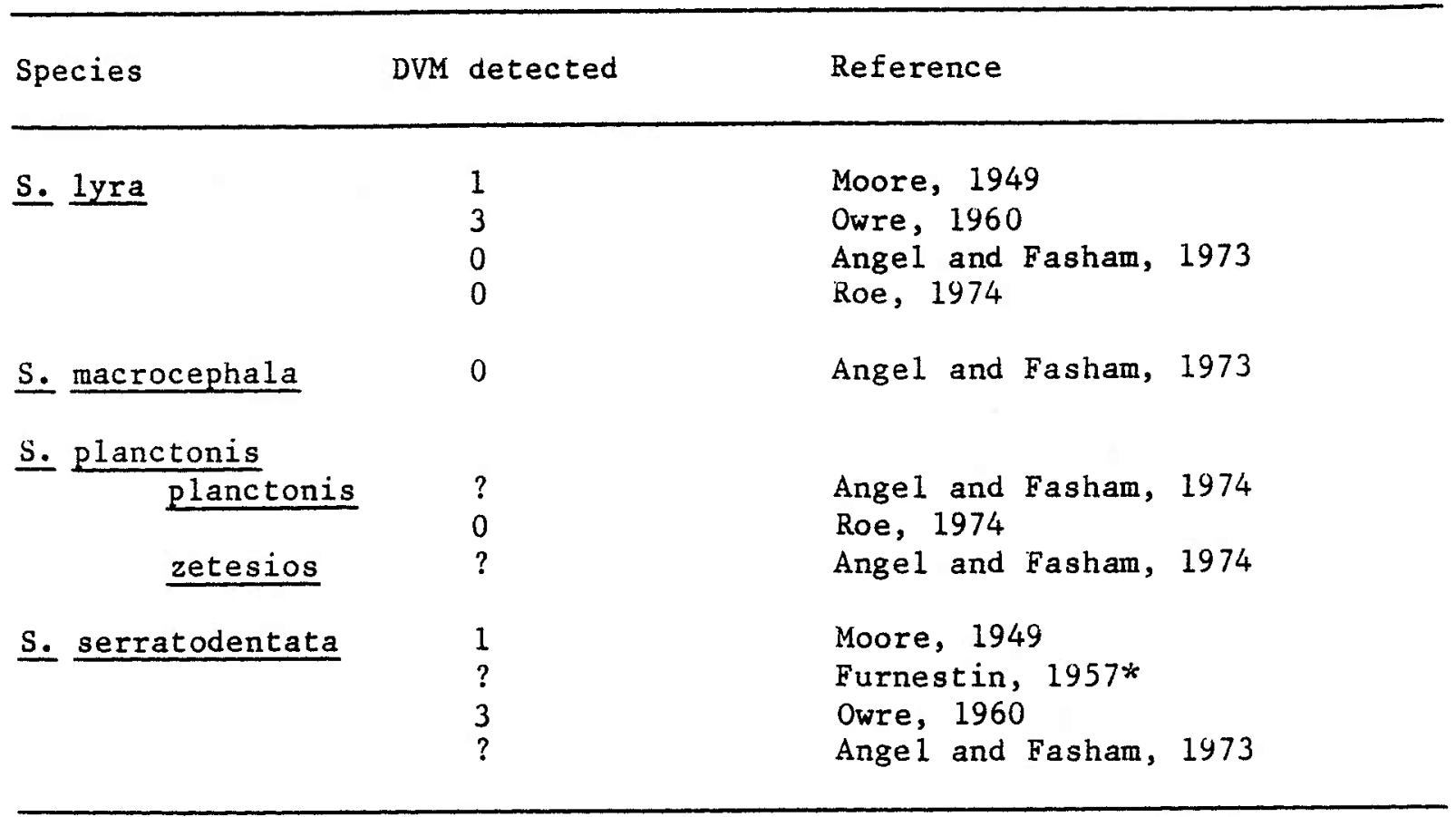

* As cited by Alvarino, 1965. 
chaetognaths from this study are presented in Table 4.7. Perhaps the only chaetognath for which diel vertical migration has been well documented is S. elegans (Russel1, 1931; Pearre, 1973). The only reports of extensive ( $>$ $100 \mathrm{~m})$ diel vertical migrations for chaetognaths cone from Owre's (1960) study in the Florida Current. These results should be viewed with caution, however, as the observations were made at the SL station, and the large differences in vertical distribution were probably related to the downwelling phenomenon reported by Stepien (1980). Waterman and Berry (1967) provided the only report of diel vertical migration for bathypelagic chaetognaths (Eukrohnia spp.). There was clearly no diel vertical migration detectable for any of the bathypelagic species in the present study, and I suggest that Waterman and Berry's observations were due to inadequate sampling techniques. Welsh et a1. (1937) reported diel vertical migration for $\mathrm{S}$. hexaptera from a time series collection at $400 \mathrm{~m}$ in the Northern Sargasso Sea. As discussed earlier, the results of such a sampling procedure are ambiguous and should be viewed with caution.

The reports by Moore (1949), Kolosova (1972), and Pearre (1974) are the best evidence available for diel vertical migrations for the species considered here. Their studies were limited mainly to epipelagic species, although Moore reported the mesopelagic species $\underline{K}$. subtilis and $\underline{S}$. $\underline{1 y r a}$ to be migrating within the upper $25 \mathrm{~m}$ of the water column.

From the results of this study and published reports, it may be concluded that there is little or no evidence of diel vertical migrations by chaetognaths from below $200 \mathrm{~m}$. The evidence suggests that diel vertical migrations by chaetognaths are of limited extent $(<100 \mathrm{~m})$ and occur primarily within the upper $200 \mathrm{~m}$ of the water column. 
Table 4.8. Reports of ontogenetic migrations of chaetognaths. 
Species for which ontogenetic migrations have been detected

Species

E. bathypelagica

E. fowleri

E. hamata

K. subtilis

P. draco

S. bedoti

S. decipiens

S. elegans

S. enflata

S. gaze1lae

S. hexaptera

S. Iyra

S. macrocephala

S. marri

S. maxima

S. $\underline{\text { minima }}$

S. planctonis

S. regularis/neglecta

\section{Reference}

Nair, 1978

Alvarino, 1964; Nair, 1978

Fowler, 1905*; Kramp, 1917*; Alvarino, 1964;

Kitou, 1967; Nair, 1978; Present study

Stone, 1969; Present study

Stone, 1969

Stone, 1969

Stone, 1969; Present study

Kramp, 1917*; Huntsman, 1919; Bigelow, 1926;

Russe11, 1931; Alvarino, 1964; Jakobsen, 1971 ;

Kotori, 1972; Pearre, 1973; King, 1979; Sullivan, 1980

Stone, 1969

David, 1958; Alvarino, 1964

Stone, 1969; Present study

Furnestin, 1962*; Kitou, 1966; Stone, 1969;

Present study

Alvarino, 1964; Present study

David, 1958

Present study

Alvarino, 1964; Stone, 1969

Alvarino, 1964

Stone, 1969 
Table 4.8. (cont'd).

$\begin{array}{ll}\text { Species } & \text { Reference } \\ \text { S. scrippsae } & \text { Alvarino, } 1964 \\ \text { S. serratodentata } & \text { Stone, } 1969\end{array}$

Reports of no observed ontogenetic migrations

Reference $\quad$ Species

Owre, 1960 K. pacifica, K. subtilis, P. draco, S. bipunctata,

S. decipiens, s. enflata, s. hexaptera, s. lyra,

S. minima, s. serratodentata

Stone, 1969 S. bipunctata, S. robusta/ferox

Pearre, 1974 S. friderici, S. enflata, S. minima

Lyons, 1976 K. pacifica, K. subtilis, P. draco, S. bipunctata,

S. decipiens, S. enflata, S. ferox, S. hexaptera,

S. maxima, S. minima, S. pacifica, s. robusta, s. pseudoserratodentata

* As cited by Alvarino, 1964 . 
Ontogenetic migrations have been reported for a number of epi-, meso-, and bathypelagic chaetognaths (Table 4.8). Owre (1960) reported, "...no especial concentrations of juveniles were found in the upper layers even at times when spawning appeared to have been heavy...." The sporadic downwelling events reported by Stepien (1980) again may have altered the patterns so that such migrations were not detectable. However, since there are few reports of ontogenetic migrations by epipelagic species and Owre's species were mostly epipelagic, her results may accurately reflect the behavior of those species. Lyons (1976) reported, "Vertical distributions of juveniles and adults for each of the species did not differ in any obvious way..." A careful examination of his data suggests that juveniles were shallower than adults in either summer or winter for $\underline{K}$. subtilis, $\underline{P}$. draco, S. bipunctata, S. decipiens, S. hexaptera, S. maxima, and S. pacifica.

These reports suggest that ontogenetic migrations are a widespread behavior among chaetognaths, especially meso- and bathypelagic species. Further studies are needed to determine the extent of these migrations and the relationships of size and sexual maturity classes to environmental conditions. The adaptive value of these migrations has received little attention and deserves further consideration.

Vinogradov (1970) proposed that diel and ontogenetic migrations may be important means for transporting food (organic matter) to greater depths in the ocean. Although diel vertical migrations by chaetognaths are probably insignificant in this regard, the extensive ontogenetic migrations by the meso- and bathypelagic species may be quite important in transferring organic matter to the ocean depths. 
Regulation of the vertical distribution of chaetognaths

A review of mechanisms suggested by previous authors for controlling the vertical distributions of chaetognaths can provide some insight into the problem. The two variables which are most frequently invoked as regulating zooplankton vertical distributions are temperature and light.

That temperature affects biochemical and physiological processes of all organisms is well documented. It is also clear that no poikilothermic species can be adapted for growth and reproduction at all temperatures found in the oceans. On this basis, it has long been assumed that temperature must play a significant role in regulating the vertical distributions of zooplankton.

Moore et a1. (1953) found a significant correlation $(r=0.24, p<0.05)$ of median depth for nine chaetognath species with the depth of the $15^{\circ} \mathrm{C}$ isotherm in the Florida Current. No significant correlation of median depth with light was found, nor was there a significant correlation of median depth with season. The $25 \%$ depth level of these species was correlated more with light than temperature, and the reverse was true for the $75 \%$ depth level. That upper and lower members of the population responded differently may be related to ontogenetic migrations and the different responses of size or sexual maturity classes. They also tested the hypothesis that increases in the distance between the isotherm and isolume used resulted in a larger spread of the population ( $75 \%$ depth level - $25 \%$ depth level) and found a significant correlation coefficient of 0.63 .

In a later paper, Moore (1955) calculated partial regressions of the 
10,50 , and $90 \%$ depth levels on the depth of the same isotherm and isolume used earlier for the same nine chaetognaths. Comparisons of predicted 10, 50, and $90 \%$ depth levels with observed values were quite good. Owre (1960), in a more detailed analysis of individual species' vertical distributions from the same data, concluded that temperature was primarily controlling the median depth of most of the species, with light and pressure having modifying effects for some species. These observations from the Florida Current cannot be regarded as conclusive, however, because of the downwelling events discussed by Stepien (1980).

The submergence of bathypelagic chaetognaths in temperate regions discussed earlier suggests that temperature regulates their vertical distributions. Banse (1964), however, argued that these species may simply remain with a particular water mass as it submerges. Alvarino (1964, 1965) also discussed the submergence and emergence of chaetognaths as related to water mass movements. It is difficult, if not impossible, to determine whether the observed patterns are the result of passive distribution processes or active behavioral responses to temperature. The results of the present study suggest another hypothesis. That is, the observations of submergence are the result of ontogenetic migrations and population size structure differences between regions. This hypothesis is easily tested and should be given further consideration.

Light probably has no direct effect on mortality of zooplankton except with regard to visually directed predation. Thus, light is best viewed as providing a means of orientation for these organisms. As seen from the light profiles in Figure 4.1, light provides a reasonable estimate of depth in both the SW and NSS. Within the upper $100 \mathrm{~m}$, noon light of a 
specified intensity varied in depth $\pm 25 \mathrm{~m}$, and below $100 \mathrm{~m}$ noon light of a specified intensity varied in depth $\pm 50 \mathrm{~m}$. Although there has been much specualtion regarding the control of vertical distributions by light, no direct relationship of vertical distribution of a particular species with a particular light intensity has ever been reported. A large part of the problem may be that light is usually indirectly calculated by previous workers, as was done in this study. Rigorous testing of the hypothesis that zooplankters follow isolumes will require direct measurement of light during the sampling of the vertical distributions.

Russe11 (1931) discussed the role of light in determining the vertical distributions of several sexual maturity stages of $\underline{\mathrm{S}}$. elegans, but he could find no correspondence of average depth with the depth of a particular isolume. As discussed earlier, Moore et al. (1953) found no correlation of light and median depth for nine chaetognath species, but light appeared to have some effect in determining the spread of the populations.

Singarajah (1966) reported a pressure sensitivity for the chaetognath S. setosa. It swam upward in response to increases in pressure equivalent to $2 . j \mathrm{~m}$ of seawater, but it is not clear if this pressure sensitivity can be used for determination of depth.

oxygen concentration has occasionally been invoked as regulating the horizontal and vertical distributions of zooplankton. Alvarino (1964) reported that $\underline{\mathrm{S}}$. elegans was confined to waters with greater than $6 \mathrm{ml} / 1$ of oxygen. Subsequent authors have reported $\underline{S}$. elegans from waters with oxygen concentration as low as $0.1 \mathrm{ml} / \mathrm{l}$ (Kotori, 1972; Jakobsen, 1971; Sullivan, 1980). Alvarino (1965) also reported that the vertical 
distribution of S. decipiens was related to oxygen concentration in the Gulf of California. This is quite plausible, as oxygen concentrations below $0.1 \mathrm{ml} / 1$ have been reported from intermediate depths there (Sverdrup et al., 1942). It is not likely that oxygen concentration has any effect on the horizontal or vertical distributions of chaetognaths in the western North Atlantic Ocean because miminum concentrations are about $3.0 \mathrm{ml} / 1$ (Sverdrup et al., 1942) (Figure 4.1).

It also appears unlikely that salinity variations observed in oceanic waters have much effect on zooplankters. Experiments performed by Hopper (1960) suggested that oceanic zooplankters are tolerant of moderate (10-20\%) decreases of salinity. Zooplankters were much less tolerant of salinity increases, however. Harder (1968) observed that many zooplankters aggregate at salinity discontinuities in laboratory experiments, but this was shown to be a response to vertical density differences rather than to salinity differences for the copepod Temora longicornis. This species showed no aggregation at a salinity discontinuity when density differences between layers were eliminated by the addition of sugar. Salinity values ranged from 32.8 to 36.8 ppt in the present study (Figure 4.1), so there should be little or no effect of salinity on the observed vertical distributions.

Sullivan (1980) and Ortner et al. (1980) suggested that vertical patterns of food abundance may affect the vertical distributions of chaetognaths. It is not clear whether this is a response to increased concentrations of food per se or to other environmental cues which indicate where food should be most abundant. Harder (1968) suggested that aggregation by zooplankters at density interfaces is adaptive because 
particulate matter (i.e. food) collects at such surfaces. The relationship of chaetognath abundance with the deep chlorophyl1 maximum reported by Ortner et al. may have been due to the chaetognaths' detection of the pycnocline with which the deep chlorophyll maximum was associated.

As discussed earlier, vertical water movements may also affect the vertical distributions of chaetognaths.

\section{Conclusions}

The hypothesis that all members of a population have essentially the same response to environmental conditions in selecting their vertical distributions is not supported by the data presented here. The ontogenetic migrations observed for the meso- and bathypelagic chaetognaths clearly indicate that the response to environmental conditions depends upon the size of the individual, or some correlated characteristic such as age or sexual maturity. McLaren (1969) realized this when he wrote, "...differences between developmental stages of a species may be just as great as differences between species; it is not possible to generalize about diurnal, seasonal, or geographical differences in depth of a species from studies of all stages combined or of a single stage, although there are many such studies in the literature". If we are to understand how the vertical distributions of chaetognaths are regulated, it will be necessary to examine the vertical distributions of size or possibly sexual maturity classes in relation to temperature, light, etc. As mentioned earlier, such an analysis is currently underway for S. lyra. 
Much better sampling of the upper $200 \mathrm{~m}$ is needed to understand the depth regulation of the epipelagic chaetognaths. Whether or not these species undertake diel or ontogenetic vertical migrations must also be more carefully examined.

Although laboratory studies can provide insight into the mechanisms involved in this habitat selection by chaetognaths, such studies are limited by the difficulties of keeping the organisms alive and recreating the wide range of environmental conditions as a function of depth found in nature. For these reasons, I believe that field studies will continue to provide the most useful information for these purposes. Improved sampling gear and a better knowledge of the errors associated with sampling enable us to more accurately assess the relationships of vertical distributions with environmental conditions. Sampling in quasi-experimental regions, such as Gulf Stream cold and warm core rings, provides informatio on the changes in vertical distribution that accompanies the changes in environmental conditions with time. Eddies similar to those formed by the Gulf Stream have also been observed in other regions with strong currents such as the Kuroshio Current, the Somali Current, the Agulhas Current, and the East Australia Current (O1son and Wiebe, 1981).

Understanding the regulation of the vertical distributions of zooplankton is a prerequisite for understanding their horizontal and temporal abundance patterns. Variables such as temperature and quality and quantity of food are known to affect the population dynamics of zooplankters, and the values of these variables change rapidly with depth in the oceans. 


\section{CHAPTER 5}

Horizontal and Seasonal Abundance Patterns

of Chaetognaths

in the Western North Atlantic Ocean 


\section{Introduction}

The broad geographic distributions of chaetognaths are well known (Bieri, 1959; A1varino, 1965), but as the reported ranges are based primarily on presence/absence data, they include expatriated populations as well as self reproducing (= independent) populations. Expatriated populations may be found at great distances from the home range of the independent population(s) (McGowan, 1960), and the inclusion of expatriated with independent populations in the published ranges obscures the relationships between these species and the environmental conditions necessary to support their populations. In order to develop testable hypotheses regarding the regulation of the geographic abundance patterns, it will be necessary to distinguish between independent and expatriated populations.

The region of the western North Atlantic south of Cape Cod, Massachusetts is a region of sharp horizontal gradients in hydrography and species composition. The Gulf Stream separates the colder less saline SW from the warmer, more saline NSS. Previous studies have characterized some of the differences in phytoplankton, zooplankton, and midwater fish species composition between the SW and NSS (Ortner et al., 1979; Grice and Hart, 1962; Jahn and Backus, 1976). Alvarino (1965, 1969) depicted the SW/NSS region as the northern limit for many subtropical chaetognaths and the southern limit for several temperate and boreal species. Grice and Hart (1962) observed that many of the chaetognaths in the upper $200 \mathrm{~m}$ were common to both the SW and NSS, but there were abundance differences for those species inhabiting both regions. While the chaetognath species 
composition for these regions is well known, it is not at all clear whether these species are present as expatriated or independent populations.

Previous studies of chaetognath distributions within the SW/NSS region have not appreciated the contributions of Gulf stream rings to the mixing of SW and NSS populations. In the past several years, it has become increasingly clear that Gulf stream rings are an important source of mesoscale biological, chemical and physical variability in this western North Atlantic (Wiebe et al., 1976b; Ortner et a1., 1978; The Ring Group, 1981).

The purpose of the present study was to describe the spatial and temporal abundance patterns of chaetognaths in the upper $1000 \mathrm{~m}$ in relation to the hydrography of the SW/NSS region. Spatial and temporal abundance patterns from a limited set of ring observations are also described.

Stone (1969) suggested that community analysis (winich I will refer to as species association analysis) may provide additional insight into the spatial and temporal abundance patterns of zooplankton. Several techniques of species association analysis are compared as applied to the chaetognath data from this study.

It is suggested that these hydrographic abundance patterns may be used to distinguish independent from expatriated populations. Habitat differences between the SW and NSS are described, and several hypotheses regarding the regulation of the observed abundance patterns are discussed. 
Methods

Zooplankton samples were collected and chaetognaths were identified as described in Chapter 2. Hydrographic and temporal abundance comparisons were based upon the number of individuals of each species under a square meter of ocean surface $\left(n o . / \mathrm{m}^{2}\right)$ in the upper 800 or $1000 \mathrm{~m}$ of the water column, depending upon the maximum depth of sampling. Some of the bathypelagic species occurred in large numbers below $800 \mathrm{~m}$, so the abundance data for these species from the 0-800 $\mathrm{m}$ tows were excluded from all analyses except the species association analysis and the temporal analysis from Ring D (see below).

It must be remembered that the abundances presented do not represent the total population abundances because of sampling errors as discussed in Chapter 3. The most important of these errors appeared to be mesh selection, with the result that individuals smaller than about $6 \mathrm{~mm}$ were not well represented in the samples. There may also have been some avoidance by the larger $(>20 \mathrm{~mm})$ individuals of the larger species. In spite of the underestimation of total abundance, I believe that the observed abundances reflect the spatial and temporal patterns of the total population abundances. Appropriate statistical tests for abundance differences were used, thereby accounting for the effects of patchiness and subsampling.

Tows were classified as representing SW, NSS, GS, and cold core rings on the basis of vertical profiles of temperature and salinity. Ring $D$ was sampled in August (three tows) and November (two tows) of 1975, and Ring Bob was sampled in August of 1977. 
Three methods were used in the species association analysis. Recurrent group analysis is a method based upon the grouping of species which co-occur frequently in a set of tows (Fager, 1957; Fager and McGowan, 1963). The number of joint occurrences $(J)$ is used in the calculation of an affinity index (I) between all species pairs:

$I=J /\left(N_{A} N_{B}\right)^{1 / 2}-N_{B}^{1 / 2} / 2$,

where $\mathrm{N}_{A}$ and $\mathrm{N}_{B}$ are the total number of occurrences of species $A$ and $B$ $\left(N_{A} N_{B}\right)$. The tirst term in the equation represents the geometric mean of the proportion of joint occurrences and the second term is a correction for sample size. Fager and McGowan (1963) considered values of I greater than 0.5 to represent affinity between species, and that value was used in the present study. Species groupings were determined from procedures outlined by Fager (1957).

Several methods are available for analyzing quantitative relationships between species in a set of tows. These methods fall into the categories of cluster analysis or ordination analysis. There is controversy over what the best clustering procedures are (Williams, 1971), and it can often be difficult to determine the relationships between species in different clusters. Ordination methods, on the other hand, ideally allow the simultaneous viewing of relationships between all species. These methods attempt to reduce the relationships between variables in a large data set to a smaller, more easily interpretable set of values. I have applied two ordination methods, correspondence analysis (CA) and factor analysis (FA), to the chaetognath abundance data. 
$\mathrm{CA}$ is most easily viewed by this author as a partitioning of the chi-square statistic which can be computed for a species by tow abundance matrix. The total chi-square is partitioned into an uncorrelated set of factors, and each species is assigned a factor score for each factor based upon the species' contribution to the chi-square accounted for by each factor. Ideally, the first two or three factors will account for most of the total chi-square, so that two or three dimensional plots of the species by their factor scores will reveal the important relationships between species. Rigorous mathematical presentations of CA are provided by Hill (1974), Teil (1975), McElroy and Smith (1975) and Chardy et al. (1976).

CA offers two important advantages over other ordination techniques. It assumes no underlying statistical distribution of the data (David et a1., 1974) and it simultaneously orders both species and tows (Hill, 1973). Computer routines used for $\mathrm{CA}$ were developed by J. P. Bordet of the Ecolie Nationale Superiere des Mines de Paris, Centre d'Informatique Geologique and subsequently modified by W. K. Smith at WHOI.

FA partitions the variance of the species abundances into a set of uncorrelated factors in a manner analogous to the chi-square partitioning of CA. Species abundances are first standardized by production of a species by species correlation matrix. An interesting feature of FA is that each species variance is first separated into comon and unique components, and then only the common variance components for each of the species are partitioned. The common variance components are those shared with other species in the analysis, while the unique component for each species is not shared with other species. The factor analysis model is 
somewhat attractive in this regard, as species, by definition, have unique characteristics, some of which may result in unique responses to environmental conditions. Again, factor scores are assigned to each species for each factor based upon the species' contribution to that factor. There are a number of good mathematical presentations of FA, including Harman (1967) and Rummel (1970).

I used the SPSS subprogram FACTOR using PA2 factoring and varimax rotation (Nie et al., 1975). FA assumes a multivariate normal distribution of the species abundances for the calculation of correlation coefficients, an assumption clearly not satisfied by the present data (see Chapter 3 ). Therefore, both untransformed and $\log _{10}(X+1)$ transformed species abundances were used.

Hydrography and habitat differences in the western North Atlantic

Iselin (1936) recognized four distinct bands of water as proceeding seaward from the northeastern U.S. coast. These bands of water are now recognized as the shelf water, the slope Water, the Gulf Stream, and the Northern Sargasso Sea (Figure 5.1). These hydrographic regions are easily identified on the basis of vertical profiles of temperature and salinity. There can be large fluctuations in the geographic positions of the boundaries between these regions, so a knowledge of the hydrography is essential for understanding the spatial and temporal abundance patterns of zooplankton in this region. I will first present a brief review of the hydrography of the SW/NSS region, and I will then discuss important 
Figure 5.1. Hydrography of the western North Atlantic. 


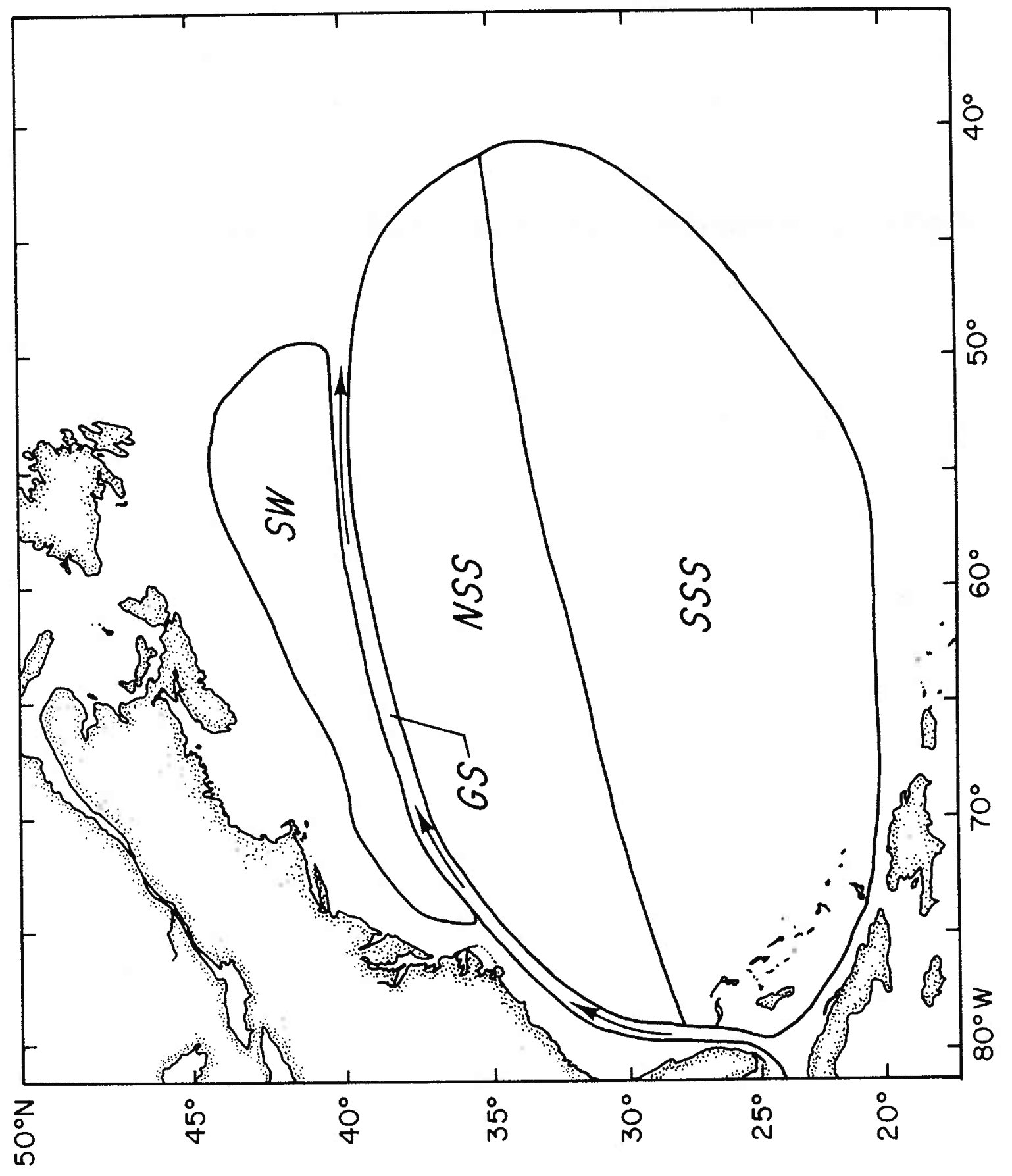


habitat differences between the SW and NSS.

The shelf water south of Cape Cod generally extends seaward to the 100 m isobath (Wright, 1976a). Surface shelf water frequently overlies the SW near the shelf/slope boundary. Shelf water salinity values generally range from 32 to 35 ppt (Wright and Parker, 1976). The shelf water is somewhat continuous from Nova Scotia to Cape Hatteras, and currents are weak and variable with mean flow to the southwest (Wright, 1976a). Wright (1976b) reported the frequent occurrence of detached shelf water parcels in the SW region.

The SW is also continuous from Nova Scotia to Cape Hatteras. Lee (1970) offered this definition of SW:

"The slope water is the body of water, from the surface down to approximately $900 \mathrm{~m}$, that generally lies within the region bounded by the edge of the continental shelf and the Gulf Stream. Its surface water is the incompletely mixed product of Coastal Water and Gulf Stream Water, and its subsurface water is the incompletely mixed product of Labrador Water and Atlantic Water."

Lee's Atlantic Water is equivalent to Iselin's (1936) North Atlantic Central Water (NACW) and Worthington's (1976) Western North Atlantic Water. The upper $200 \mathrm{~m}$ of the $\mathrm{SW}$ is primarily a mixture of shelf water and Gulf Stream water (McLellan, 1957; Lee, 1970). Shelf water is probably supplied by the detached parcels observed by Wright (1976a), and while GS water may be blown into the SW by southerly winds, Gulf stream warm core rings are probably the major source of GS water (Wright, 1976b). NSS 
surface water is also brought into the SW region by warm core rings. Wright (1976b) estimated a residence time of about a year for the surface SW.

Surface salinity varies from about 34 to 36 ppt and surface temperature varies both spatially and seasonally in the upper $200 \mathrm{~m}$ of the SW (Wright, $1976 b)$.

Below $200 \mathrm{~m}$, the $\mathrm{SW}$ is a mixture of Labrador Water and NACW (Iselin, 1936; McLellan, 1957; Lee, 1970). SW of a specified temperature is found approximately $500 \mathrm{~m}$ deeper in the adjacent NSS. McLellan (1957) and Lee (1970) estimated that eastern SW is about $80 \%$ NACW and $20 \%$ Labrador Water. The NSS appears to be the source of NACW for the SW. NACW may be pumped across the Gulf stream along equal density surfaces according to Rossby's (1936) wake stream theory, but warm core rings probably supply much of the NACW to the SW. Wright (1976b) cited three reports of anomalously cold water at intermediate depths in the SW. Colder, fresher Labrador Water may occasionally replace large volumes of $\mathrm{SW}$, but conditions appear to be restored to normal within a year.

Few current measurements are available from the SW, but what data are available suggest a slight and variable counterclockwise circulation in the western SW (Wright, 1976b), and a somewhat stronger clockwise flow in the eastern SW (Lee, 1970; Worthington, 1976). In the deep water $(>1000$ m), the Western Boundary Undercurrent transports North Atlantic Deep Water to the southeast and under the Gulf Stream near Cape Hatteras. The Gulf Stream is a narrow, intensive western boundary current which transports Caribbean water and associated plankton to high latitudes in the North Atlantic. It is approximately $100 \mathrm{~km}$ wide south of Cape Cod and 
is characterized by surface currents of up to $250 \mathrm{~cm} / \mathrm{s}$ and steeply, downward sloping isotherms from the SW edge to the NSS edge. Transport by the Gulf stream is thought to extend from the surface to the bottom (Worthington, 1976), but there is still some controversy regarding the coherence of the surface and deep flows (Schmitz et al., 1981). The flow is clearly coherent in the upper $1000 \mathrm{~m}$. Some mixing with surrounding water occurs during transit northward from the Florida Straits and Sargasso Sea water appears to be the principal source of this mixed water, but occasionally shelf water or SW may be entrained along the northern edge of the Gulf Stream near Cape Hatteras (Ford et a1., 1952; Fisher, 1972).

One of the most interesting characteristics of the Gulf stream is its formation of large meanders as it turns eastward off Cape Hatteras. These meanders can pinch off fo form both cyclonic (counterclockwise flowing) and anticyclonic (clockwise flowing) rings which move into the NSS and SW, respectively. These rings will be discussed further below.

Backus et a1. (1969) summarized evidence for a physical and biological boundary in the Sargasso Sea as depicted in Figure 5.1. I will limit discussion to the Northern Sargasso Sea, where the present zooplankton samples were collected.

The NSS may be divided vertically into three layers- the deep water (< $\left.4^{\circ} \mathrm{C},>1600 \mathrm{~m}\right)$, the main thermocline $\left(4^{\circ}-17^{\circ} \mathrm{C}, 1600-500 \mathrm{~m}\right)$ and the surface waters $\left(>17^{\circ} \mathrm{C},<500 \mathrm{~m}\right)$. The deep water is composed primarily of North Atlantic Deep Water, itself a mixture of several deep waters which may also be found in small quantities in the region.

The main thermocline is composed entirely of NACW. Iselin (1939) first 
suggested that the main thermocline waters are formed at the surface in winter at various latitudes with subsequent dispersal along equal density surfaces. Stommel (1958) hypothesized that the main thermocline is formed by the slow, upward advection of deep water and the downward diffusion of heat from surface water. It is likely that both mechanisms are involved in the production of the main thermocline water, and the presence of an oxygen minimum zone at $10^{\circ} \mathrm{C}$ (Figure 4.1 ) indicates that the main thermocline water is not renewed as frequently as deep and surface waters. The surface water of the NSS is characterized by a thermostad of approximately $18^{\circ} \mathrm{C}$ water from 200 to $500 \mathrm{~m}$. This $18^{\circ}$ water is formed during the late winter and early spring in the NSS by cooling of the surface waters and subsequent mixing of the water column to several hundred meters (Worthington, 1959). The presence of isothermal water at $18^{\circ} \mathrm{C}$ to $700 \mathrm{~m}$ during the MOC $1-60,61$ collections (Figure 4.1 ) represents the formation of $18^{\circ}$ water during April of 1977 . The $18^{\circ}$ water is transported south to approximately $20^{\circ} \mathrm{N}$ latitude, and it is presumed that overlying surface waters move north to replace that transported south as $18^{\circ}$ water (Worthington, 1976).

Worthington (1976) used both geostrophic calculations and water mass properties to infer an anticyclonic (clockwise) gyral circulation system for the Sargasso Sea. Most of the transport is confined to the NSS, and there appears to be little exchange of water with adjacent regions.

The formation of Gulf Stream rings represent large scale transfers of water, salt, heat, nutrients, and biological populations between the SW and NSS (The Ring Group, 1981). It is becoming increasingly evident that such eddies play an important role in the general circulation of the 
oceans.

Rings are formed by the pinching off of accentuated Gulf Stream meanders. Cold core rings form during southward meanders, enclosing a core of cold SW within a ring of Gulf Stream water. Warm core rings are formed by northward meanders, whereby warm NSS water is enclosed by a ring of Gulf Stream water.

Cold core rings may vary in diameter from 100 to $300 \mathrm{~km}$. Although they may persist in the Sargasso Sea for two to three years, average lifespan is shorter because many of the rings coalesce with the Gulf stream as they drift to the west and southwest. Fuglister (1972) estimated that five to eight cold core rings are formed each year, and there are, on the average, eleven rings in the Sargasso Sea at any one time (Lai and Richardson, 1977). Cold core rings which do not coalesce with the Gulf Stream gradually decay until their physical, chemical and biological properties become identical with those of the surrounding Sargasso Sea.

Warm core rings also have diameters ranging from 100 to $300 \mathrm{~km}$, but they persist in the SW for periods of only six months to a year. Again, five to eight warm core rings are estimated to form each year, but because of their relatively short lifespans only three to four are generally present in the SW at any one time (Lai and Richardson, 1977). Warm core rings also move to the west and southwest where they generally coalesce with the Gulf Stream. In transit along the slope/shelf boundary, these rings have been observed to draw shelf water into the SW region (Morgan and Bishop, 1977), and this process may be responsible for the frequent observation of shelf water parcels in the SW as reported by Wright (1976a). An indication of the importance of rings can be obtained by calculating 
the volumes of water transferred relative to the total volumes of the SW and NSS. Assuming a Sw core of $100 \mathrm{~km}$ diameter, seven cold core rings per year would add $5.5 \times 10^{4} \mathrm{~km}^{3}$ of $\mathrm{SW}$ to the upper $1000 \mathrm{~m}$ of the NSS. Jahn (1976) calculated the volume of the NSS as $32.9 \times 10^{5} \mathrm{~km}^{2}$, for a volume of $32.9 \times 10^{5} \mathrm{~km}^{3}$ in the upper $1000 \mathrm{~m}$. This results in about a two percent addition to the NSS per year in the upper $1000 \mathrm{~m}$,. Assuming seven warm core rings form per year with average diameters of $150 \mathrm{~km}$ (including the Gulf stream segment) results in a transport of $1.2 \times 10^{5}$ $\mathrm{km}^{3}$ into the upper $1000 \mathrm{~m}$ of the SW. Jahn (1976) calculated the area of the SW as $4.85 \times 10^{5} \mathrm{~km}^{2}$, corresponding to a volume of $4.85 \times 10^{5}$ $\mathrm{km}^{3}$ in the upper $1000 \mathrm{~m}$. Because warm core rings coalesce relatively rapidly with the Gulf Stream, we may assume that half, or $0.6 \times 10^{5}$ $\mathrm{km}^{3}$ remains in the $\mathrm{SW}$. This amounts to a twelve percent addition to the SW per year, and indicates that warm core rings have a much larger effect on the Sw than cold core rings have on the NSS.

The semi-enclosed circulation systems of the NSS and SW permit the development and maintenance of independent plankton populations. A brief account of the major habitat differences between these two hydrographic regions follows.

Spatial and temporal variations of physical, chemical, and biological properties are much greater in the upper $200 \mathrm{~m}$ of the SW than in the NSS. Temperature at any depth is colder in the SW than the NSS, with the possible exception of surface $(0-50 \mathrm{~m})$ temperatures in late summer (Figure 4.1). Seasonal temperature fluctuations in the upper $200 \mathrm{~m}$ are greater in the SW than in the NSS (Figure 5.2). Maximum surface temperatures are about the same in both regions $\left(\sim 27^{\circ} \mathrm{C}\right)$, but minimum surface temperature 
Figure 5.2. Seasonal temperature variations in the upper $150 \mathrm{~m}$ of the Slope Water and Northern Sargasso Sea. The curves represent from top to bottom, $0,30,60,90,120$, and 150 m (after Robinson et al., 1979). 


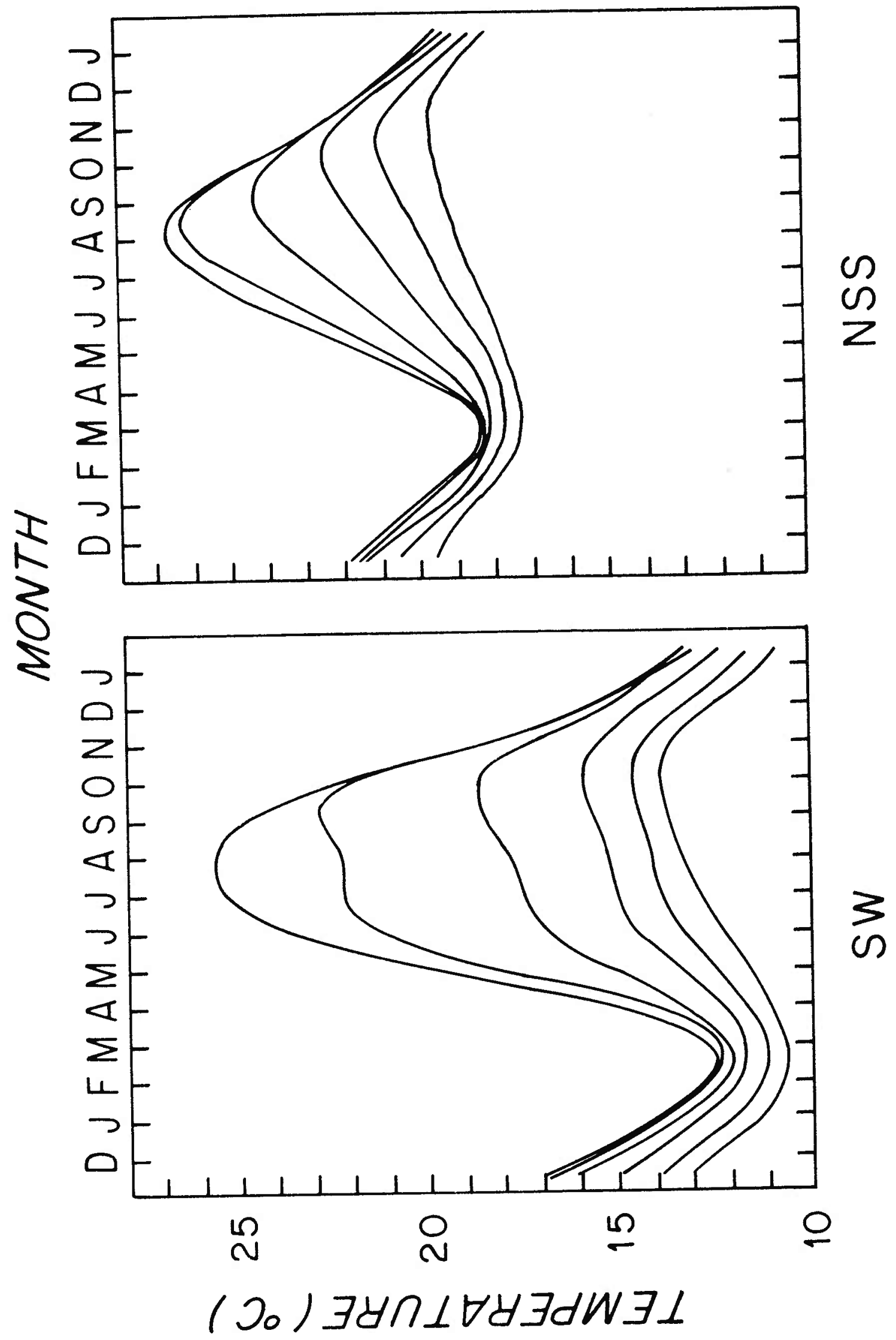


is never below $17^{\circ} \mathrm{C}$ in the NSS, yet it reaches values below $10^{\circ} \mathrm{C}$ in the SW. The formation of the seasonal thermocline is similar in both regions, with thermocline development in late spring and persistence until late fall, when surface cooling and wind mixing eliminate the seasonal thermocline. There is little seasonal variation of physical properties below $200 \mathrm{~m}$ in either region, except during $18^{\circ}$ water formation in the NSS.

Salinity and oxygen values also differ somewhat between the SW and NSS (Figure 4.1), but I am not aware of any studies which suggest that values observed for these two variables should limit the distribution of any oceanic zooplankton species.

Nutrients such as nitrate and phosphate are present in higher concentrations in the euphotic zone of the SW (Wiebe et al., 1976b). Seasonal vertical mixing processes bring large quantities of these nutrients into the surface waters during winter and early spring in both the SW and NSS.

The standing stock and productivity of phytoplankton are also greater in the SW (Wiebe et al., 1976b; Cox et al., 1982; Ryther, 1963). Phytoplankton standing stock, as measured by chlorophyll a, reaches a peak in winter and early spring in both the SW and NSS (Figure 5.3). Primary productivity also reaches a peak in the spring in the NSS, presumably as a result of the increased nutrient and light levels in the euphotic zone (Menzel and Ryther, 1961). The same seasonal pattern of primary productivity is presumed true of the SW. Ryther (1963) estimated that SW primary productivity was about twice that of the NSS $\left(120 \mathrm{gC} / \mathrm{m}^{2} / \mathrm{yr}\right.$ vs. $\left.60 \mathrm{gC} / \mathrm{m}^{2} / \mathrm{yr}\right)$. Phytoplankton species and their abundances also differ 
Figure 5.3. Seasonal patterns of chlorophyll a and zooplankton biomass in the SW and NSS. 


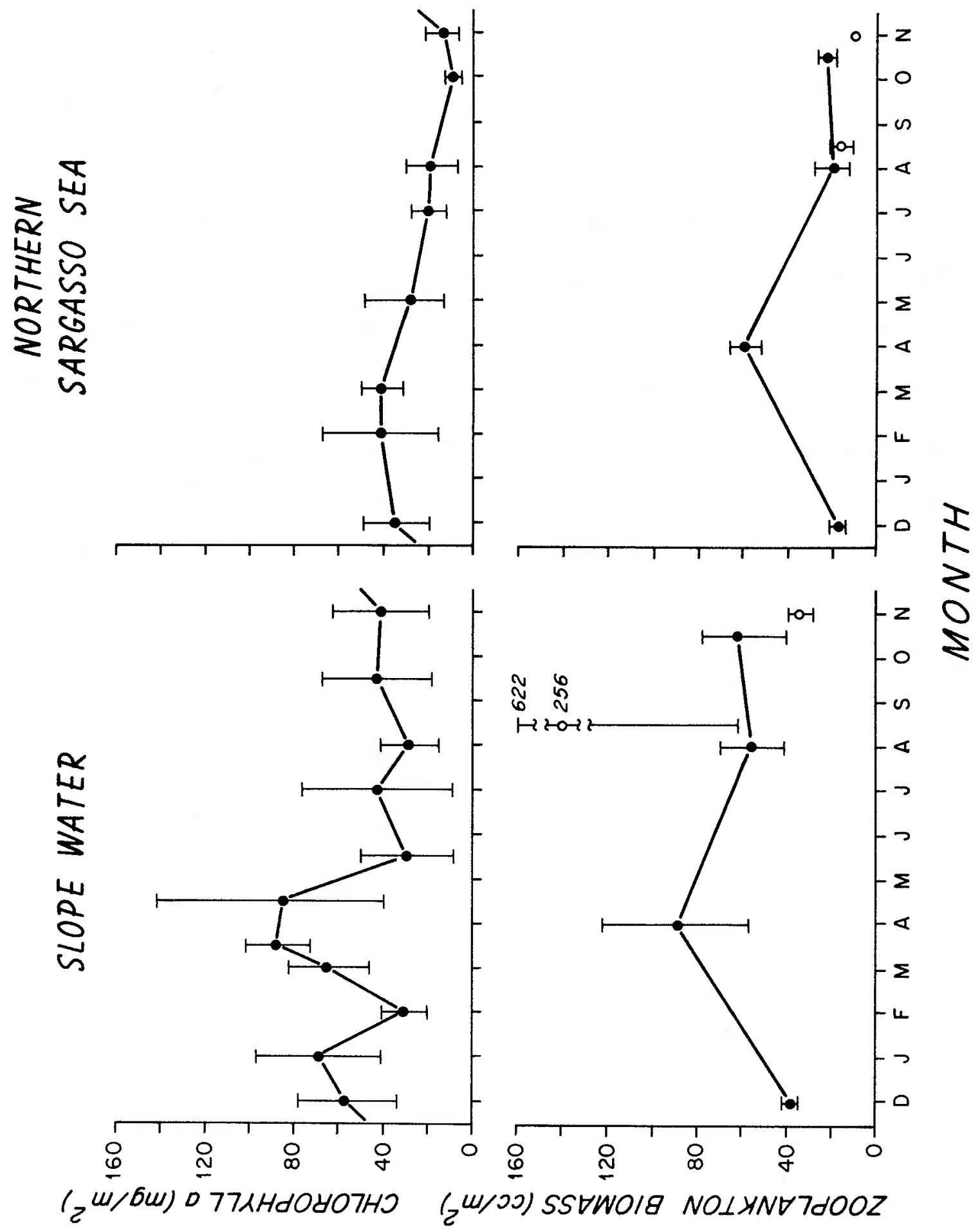


between the regions and these differences have important implications for herbivore distributions in the SW/NSS region (Hulbert, 1966; Ortner et al., 1979).

Zooplankton biomass is also greater in the SW (Be et al., 1971 provide a review of previous studies). Ortner et al. (1978) estimated that SW biomass in the upper $800 \mathrm{~m}$ was about 3.5 times that of the NSS. Peak biomass was observed in the spring in both regions during 1977 (Figure 5.3), and there was a large increase in SW biomass in August of 1975 due to large numbers of salps (Ortner et al., 1977). Such increases in SW zooplankton biomass due to salp swarms have been observed by previous investigators (Clarke, 1940; Grice and Hart, 1962). The spring biomass peak in the NSS was also documented by Menzel and Ryther (1961) and Deevey (1971), but there have been no adequate seasonal studies of zooplankton biomass in the SW. Grice and Hart (1962) reported differences in zooplankton species composition and their abundances between the SW and ISS. These differences represent changes in both the types and abundances of chaetognath prey and chaetognath predators, either or both of which may be important in regulating chaetognath abundance patterns in the SW/NSS region. 


\section{Results}

\section{Horizontal and temporal abundance patterns}

Twenty one species were identified from 52 MOCNESS tows in the SW (eighteen tows), the NSS (eighteen tows), the Gulf Stream (two tows) and Gulf Stream cold core rings (fourteen tows). Comparisons of the abundances of eighteen species in these four hydrographic regions are presented in

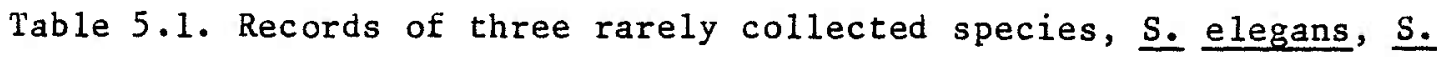

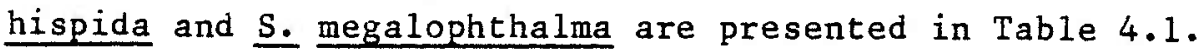

Of the eighteen species included in Table 5.1, only $\underline{\mathrm{S}}$. enflata and $\underline{\mathrm{K}}$. pacifica showed no significant abundance differences between the SW and NSS. Seven species may be classified as SW species and nine species may be classified as NSS species on the basis of their region of greatest abundance. Species abundance differences between the SW and NSS were generally quite large (Table 5.1). Twelve of the sixteen species showing significant abundance differences between the SW and NSS were greater than nine times more abundant in either the SW or NSS (Table 5.1). Although S. minima was significantly more abundant in the SW, its low NSS to SW abundance ratio (1.9) was more typical of the two species showing no significant abundance differences. E. Eowleri also had a low SW to NSS abundance ratio $(2.0)$, but this may have been due to the inability to confirm that the Eukrohnia spp. were juvenile E. fowleri. If the unidentified Eukrohnia specimens are assigned to E. fowleri, the mean SW abundance for this species becomes 36.8 individuals $/ \mathrm{m}^{2}$ and for the NSS becomes 5.4 individuals $/ \mathrm{m}^{2}$, yielding a SW to NSS abundance ratio of 6.8 . 
Table 5.1. Mean chaetognath species abundance $\left(\right.$ no. $/ \mathrm{m}^{2}$ ) in the Slope Water (SW), Northern Sargasso Sea (NSS), Gulf Stream (GS) and cold core rings (CCR). The number of tows on which the average is based is given in parentheses. $R$ represents the ratio of SW to NSS or NSS to SW abundance, whichever is larger. $P$ is the probability of equal $\mathrm{SW}$ and NSS abundances as tested by the Mann-Whitney $U$ test ( $* * *=$ $\mathrm{p}<0.001, * *=\mathrm{p}<0.01, *=\mathrm{p}<0.05, \mathrm{NS}=$ not significant $)$. 


\begin{tabular}{|c|c|c|c|c|c|c|c|c|c|c|}
\hline \multirow[t]{2}{*}{ Species } & \multicolumn{2}{|c|}{ SW } & \multicolumn{2}{|c|}{ NSS } & \multicolumn{2}{|c|}{ GS } & \multicolumn{2}{|c|}{ CCR } & \multirow[t]{2}{*}{$\mathbf{R}$} & \multirow[t]{2}{*}{$P$} \\
\hline & & Slope & Water $\mathrm{S}$ & Specie & & & & & & \\
\hline E. bathypelagica & 1.8 & (9) & 0.05 & $(14)$ & 0.74 & (2) & 0.02 & (9) & 36 & $* * *$ \\
\hline E. fowleri & 5.6 & (9) & 2.8 & $(14)$ & 3.4 & (2) & 5.7 & (9) & 2.0 & $* *$ \\
\hline E. hamata & 43.5 & (14) & 0.87 & (14) & 4.5 & $(2)$ & 30.6 & $(14)$ & 50 & $* * *$ \\
\hline S. helenae & 7.6 & (17) & 0.08 & (18) & 5.9 & (2) & 0 & (14) & 95 & * \\
\hline S. macrocepha la & 39.5 & (8) & 7.6 & $(14)$ & 29.6 & (2) & 44.9 & (9) & 5.2 & $x * *$ \\
\hline S. maxima & 21.1 & (8) & 2.0 & (14) & 3.5 & (2) & 45.5 & (9) & 11 & $* * * *$ \\
\hline \multirow[t]{2}{*}{ S. tasmanica } & 339 & (17) & 0.30 & $(18)$ & 60.5 & (2) & 69.3 & $(14)$ & 1130 & $* * *$ \\
\hline & \multicolumn{4}{|c|}{ Northern Sargasso } & \multicolumn{2}{|c|}{ Sea Species } & & & & \\
\hline K. subtilis & 3.2 & $(15)$ & 103 & $(16)$ & 23.9 & (2) & 31.0 & (14) & 32 & $* * * x$ \\
\hline P. draco & 4.9 & (17) & 160 & $(18)$ & 75.1 & (2) & 29.6 & $(14)$ & 33 & $* * *$ \\
\hline S. bipunctata & 3.7 & $(17)$ & 42.9 & $(18)$ & 14.6 & (2) & 33.6 & (14) & 12 & $* * x$ \\
\hline S. decipiens & 14.7 & $(16)$ & 150 & (18) & 90.3 & (2) & 144 & $(14)$ & 10 & $x * x$ \\
\hline S. hexaptera & 3.2 & $(17)$ & 101 & (18) & 38.5 & (2) & 34.9 & (14) & 32 & $\neq * x$ \\
\hline S. lyra & 8.9 & (15) & 179 & (18) & 51.6 & (2) & 56.5 & $(14)$ & 20 & $* * * x$ \\
\hline S. minima & 66.0 & $(18)$ & 110 & $(18)$ & 21.7 & (2) & 46.5 & (14) & 1.7 & $* * *$ \\
\hline S. planctonis & 2.0 & (8) & 7.6 & (14) & 3.1 & $(2)$ & 3.4 & (9) & 3.8 & $* x$ \\
\hline \multirow[t]{2}{*}{ S. serratodentata } & 36.7 & (17) & 342 & (18) & 110 & $(2)$ & 33.0 & (14) & 9.3 & $* * *$ \\
\hline & \multicolumn{4}{|c|}{ "?" Species } & & & & & & \\
\hline K. pacifica & 13.5 & $(17)$ & 24.8 & $(18)$ & 25.1 & (2) & 1.9 & $(14)$ & 1.8 & NS \\
\hline S. enflata & 252 & (17) & 118 & (18) & 106 & (2) & 143 & (14) & 2.1 & INS \\
\hline
\end{tabular}


The reduced abundances of the bathypelagic species (the three Eukrohnia species, S. macrocephala and $\underline{S}$. maxima) in the NSS may be due to submergence of a large fraction of their populations below $1000 \mathrm{~m}$ (see Chapter 4). As discussed in Chapter 4, the absence of juvenile E. hamata and S. maxima in the NSS suggests that their abundance differences were real, but additional sampling below $1000 \mathrm{~m}$ is required to substantiate these patterns.

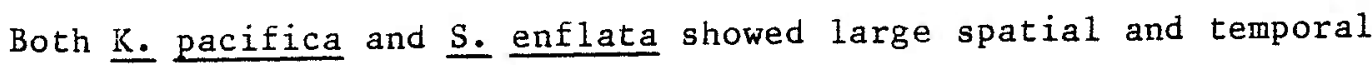
abundance variations (Table 3.12 ). K. pacifica was equally abundant in both the GS and NSS. It was one of the least abundant epipelagic species, while $\underline{\text { s. enflata }}$ was one of the most abundant.

Little can be said of the typical Gulf Stream chaetognath fauna on the basis of the two tows collected there, but chaetognath abundances were generally intermediate between SW and NSS values. Species' relative abundances were most similar to those of the NSS, although MOC 1-115 was more similar to the $\mathrm{SW}$ in relative abundance than was MOC 1-91 (Table 5.2). Vertical temperature profiles showed that MOC 1-115 was collected near the northern edge of the GS, while MOC 1-91 was collected near the southern edge (Figure 4.1). On the basis of these two tows, it can be argued that the GS chaetognath fauna is most similar to the NSS, with increasing SW influence toward the northern edge.

Species' abundances in the cold core rings were also generally intermediate between their SW and NSS abundances (Table 5.1). A more detailed discussion of chaetognath abundance patterns in cold core rings is presented later, but it may be commented here that these rings represent anomalously increased abundances of SW species and decreased 
Table 5.2. Percent similarity of the chaetognath fauna of the Gulf Stream tows with the SW and NSS. Whittaker and Fairbanks (1958) percent similarity index ranges from 0 for no similarity to 1.0 for perfect similarity. 
MOC 1-91

0.356

0.787

MOC 1-115

0.544

0.593 
abundances of NSS species in what is geographically the NSS.

Average species abundances for each cruise may best be viewed as representing their seasonal abundance patterns (Figure 5.4). Although the sampling frequency was coarse and the variability large, some general trends emerged. Included in the analysis of the temporal abundance patterns were all species from Table 5.1 except S. helenae.

In the SW, only $\underline{S}$. hexaptera and $\underline{S}$. serratodentata showed significant seasonal abundance differences (Kruskal-Wallis test applied only to the data from the 1976-77 time series cruises, $p<0.05)$. The probability of obtaining two or more significant values at the 0.05 level in seventeen tests is 0.21 , so I conclude that there were no detectable seasonal abundance differences for any species in the SW.

Seven species showed significant seasonal abundance differences in the NSS, and these were K. pacifica, S. bipunctata, S. enflata, S. hexaptera, S. 1yra, S. maxima and $\underline{S}$. serratodentata. The probability of obtaining seven or more significant differences at the 0.05 level in seventeen tests is less than 0.00001 . Six of these seven species were most abundant in April, and twelve of the total seventeen species in the analysis were most abundant in April in the NSS. Four of the five species which were not most abundant in April were bathypelagic species. The spring abundance peak for chaetognaths in the NSS corresponds with the seasonal peak of phytoplankton and zooplankton standing stock (Figure 5.3).

It is interesting to note that in the NSS nine of eleven species were more abundant in 1977 than in 1975 for both August and November (Figure 5.4). The probability of observing eighteen or more values greater in 1977 in 22 comparisons is less than 0.002 , given the null hypothesis of equal 
Figure 5.4. Seasonal abundance patterns of chaetognaths in the SW and NSS. Open circles represent 1975 cruises and closed circles represent $1976-77$ cruises. Bars indicate the ranges of abundance. 

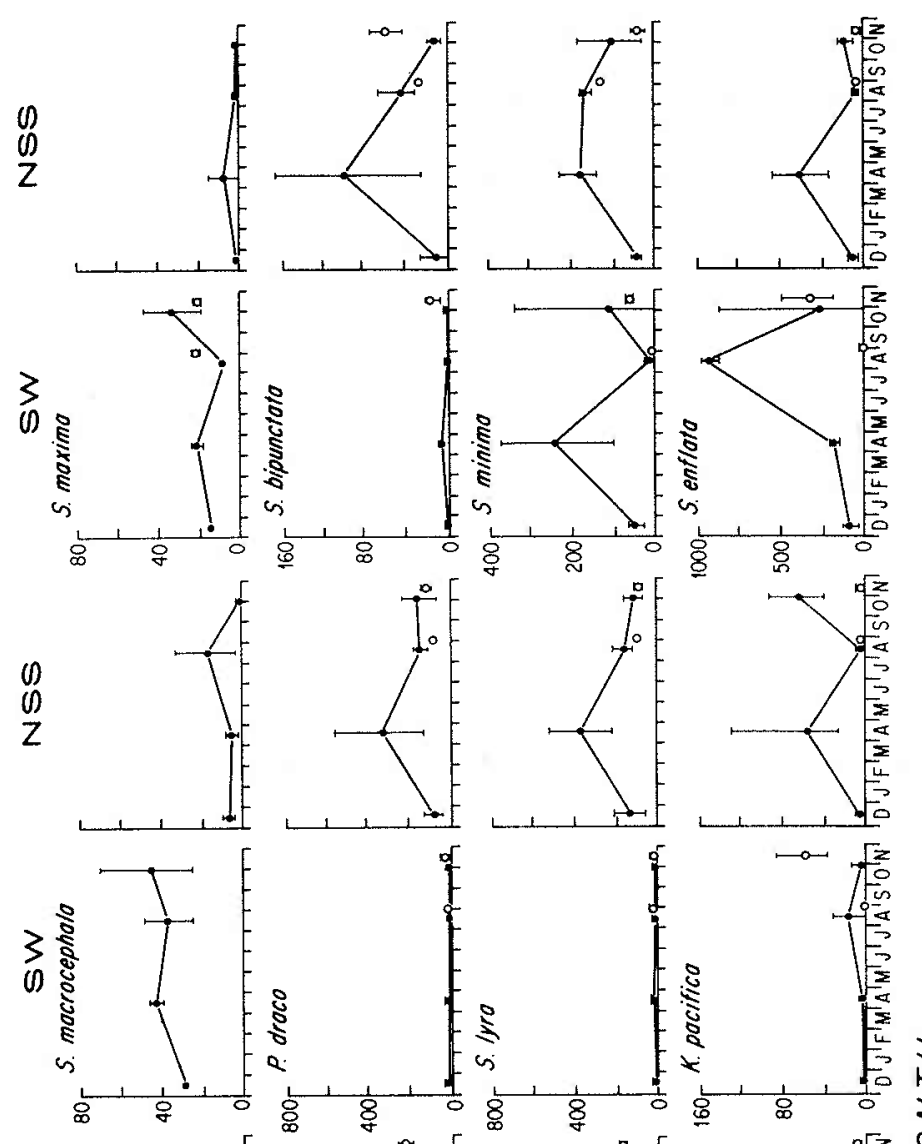

1
2
2
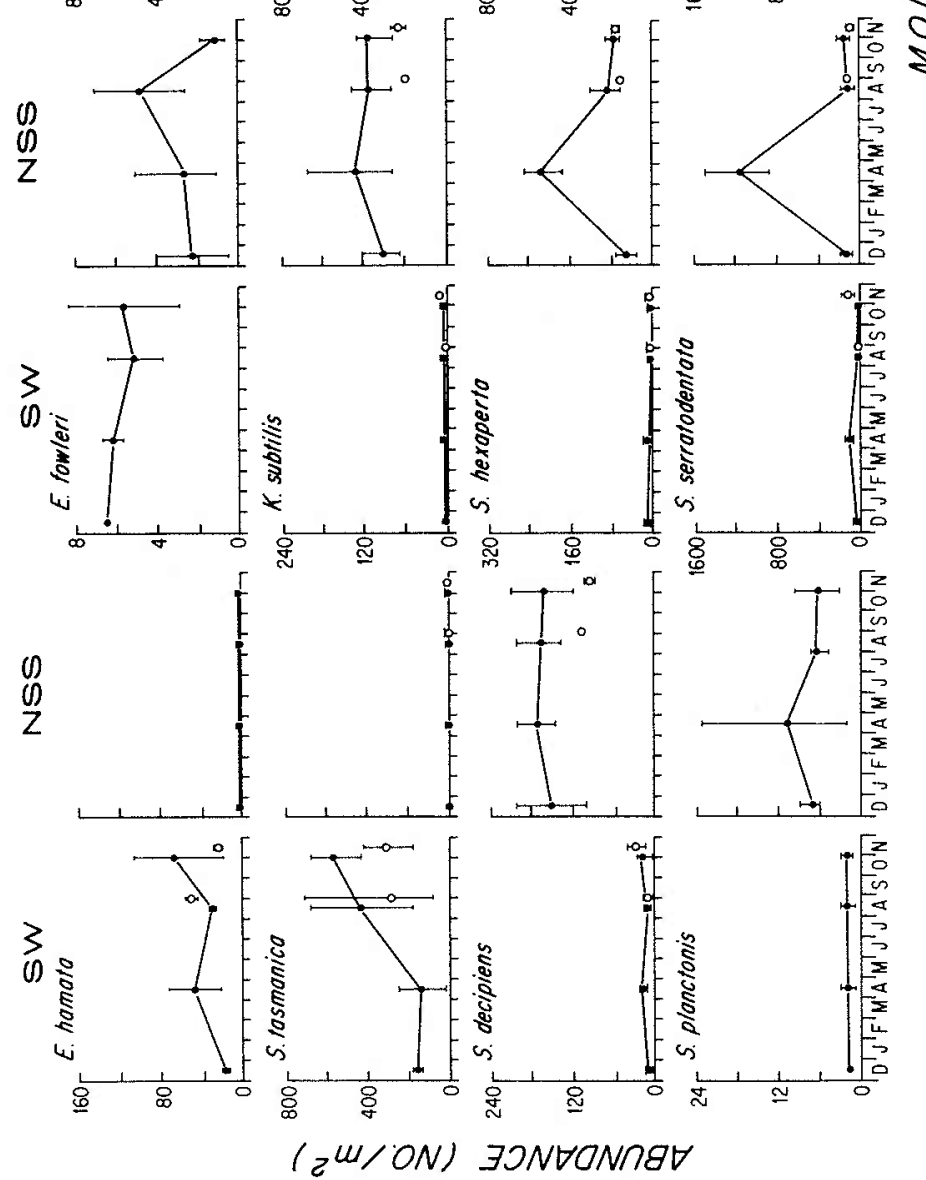
abundances in both years. This suggests that there may be year to year variations in abundance which are consistent between species, thus implying similar responses by these species to environmental conditions.

Abundance patterns in cold core rings

Abundance patterns for sixteen chaetognath species along a radial section from the center of Ring Bob into the NSS are presented in Figure 5.5. MOCNESS tows representing the SW core were numbered $77,78,84,87$ and 83 , tows representing the GS remnant (or ring fringe) were numbered $85,90,86$ and 89 , and NSS tows were numbered $82,81,80$ and 79 . Although MOC 1-82 was located near the outer edge of Ring Bob as indicated by the depths of the $10^{\circ} \mathrm{C}$ and $15^{\circ} \mathrm{C}$ isotherms (Figure 4.6), species abundances at this station were similar to those at the three NSS stations, so MOC 1-82 was also classified as a NSS station.

Species abundances along the section were remarkably similar to patterns predicted from the SW, NSS and GS abundances from Table 5.1. Lowest abundance in the ring core, increasing abundance in the ring fringe, and greatest abundance in the NSS were observed for all the NSS species except $\underline{S}$. bipunctata. For $\underline{S}$. bipunctata, maximal abundance was observed in the ring fringe, with average abundance in the ring core lower than in the NSS.

Five SW species showed the expected decrease in abundance with increasing distance from the ring core. $\underline{S}$. helenae was not present in any of these tows and $E$. bathypelagica was present in small numbers only in MOC 1-87 from the ring core. 
Figure 5.5. Spatial abundance patterns of chaetognaths in Ring Bob. 

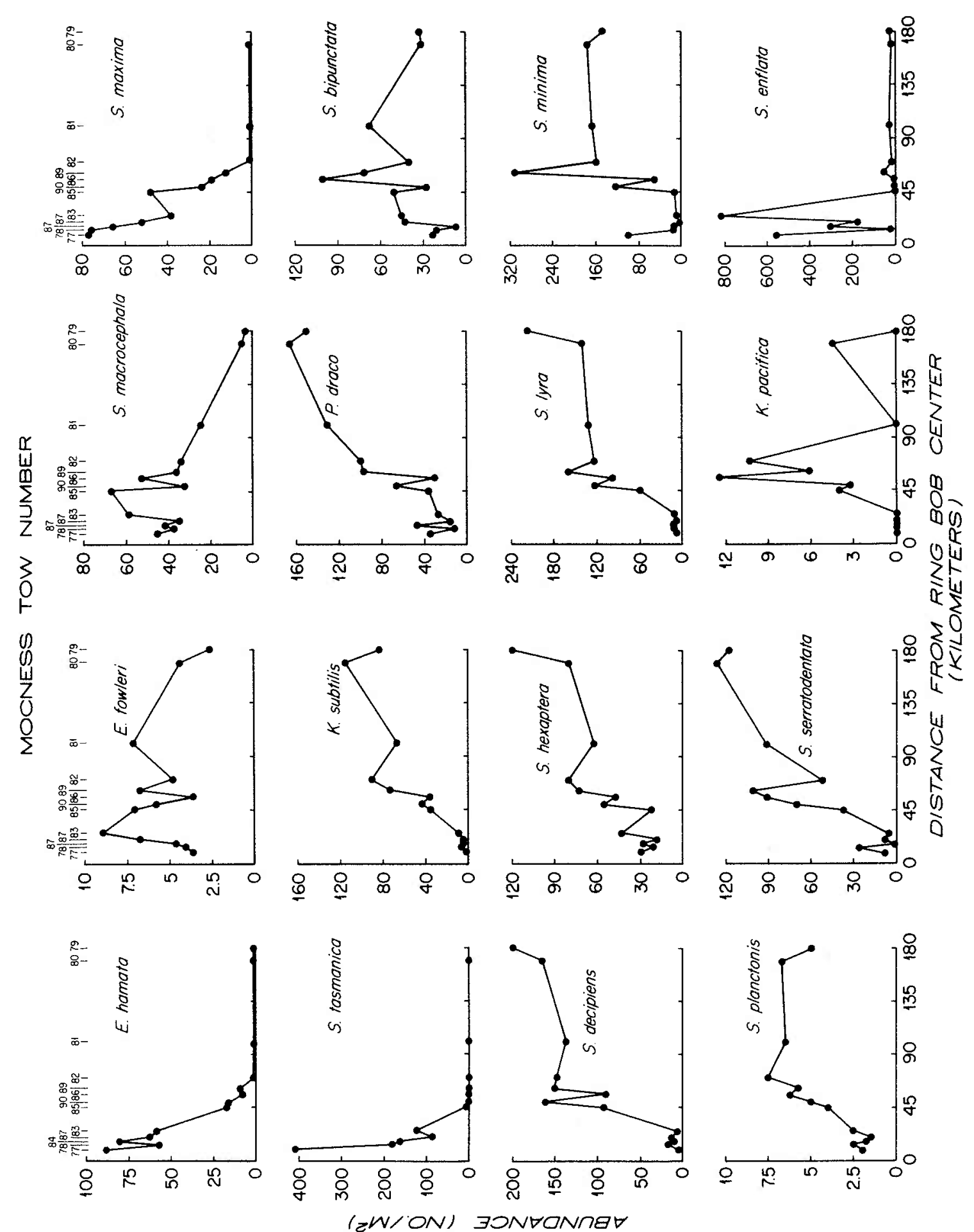

(¿W/ON) BONVONME 
K. pacifica was absent from the ring core, but was present in small numbers in the ring fringe and NSS. S. enflata abundance was both high and highly variable within the ring core, with much reduced abundance in the ring fringe and NSS. Such large variations in abundance were typical of this species in the SW/NSS region ('able 3.12 ).

Ring Bob was approximately three months old when sampled in August of 1977. The similarity of the spatial abundance patterns in Ring Bob with the patterns expected from crossing the Gulf stream in this region indicates that conditions within the ring had not changed sufficiently to affect chaetognath populations substantially. If hydrographic measurements had not been made and these stations had been classified as NSS stations, conclusions as to the species abundance differences in the SW and NSS may have been altered substantially.

Ring $D$ was sampled approximately six and nine months after its formation. Chaetognath species abundances in the ring core for August and November are plotted in Figure 5.6. Also included are average abundances in the SW and NSS to serve as approximations of the initial and final abundances in the ring. Because tows were only made to $800 \mathrm{~m}$ on the two 1975 cruises, the average SW and NSS abundances for the two bathypelagic species, E. fowleri and S. macrocephala, were calculated from only the two 1975 cruises. For the remaining species, average SW and NSS abundances were taken from Table 5.1.

A simple model of temporal changes of chaetognath abundances in cold core rings as environmental conditions changed from those of the SW to those of the NSS would predict a continual decrease in abundance for the SW species and a continual increase for the NSS species. Of the nine NSS 
Figure 5.6. Temporal abundance patterns of chaetognaths in Ring $D$. 

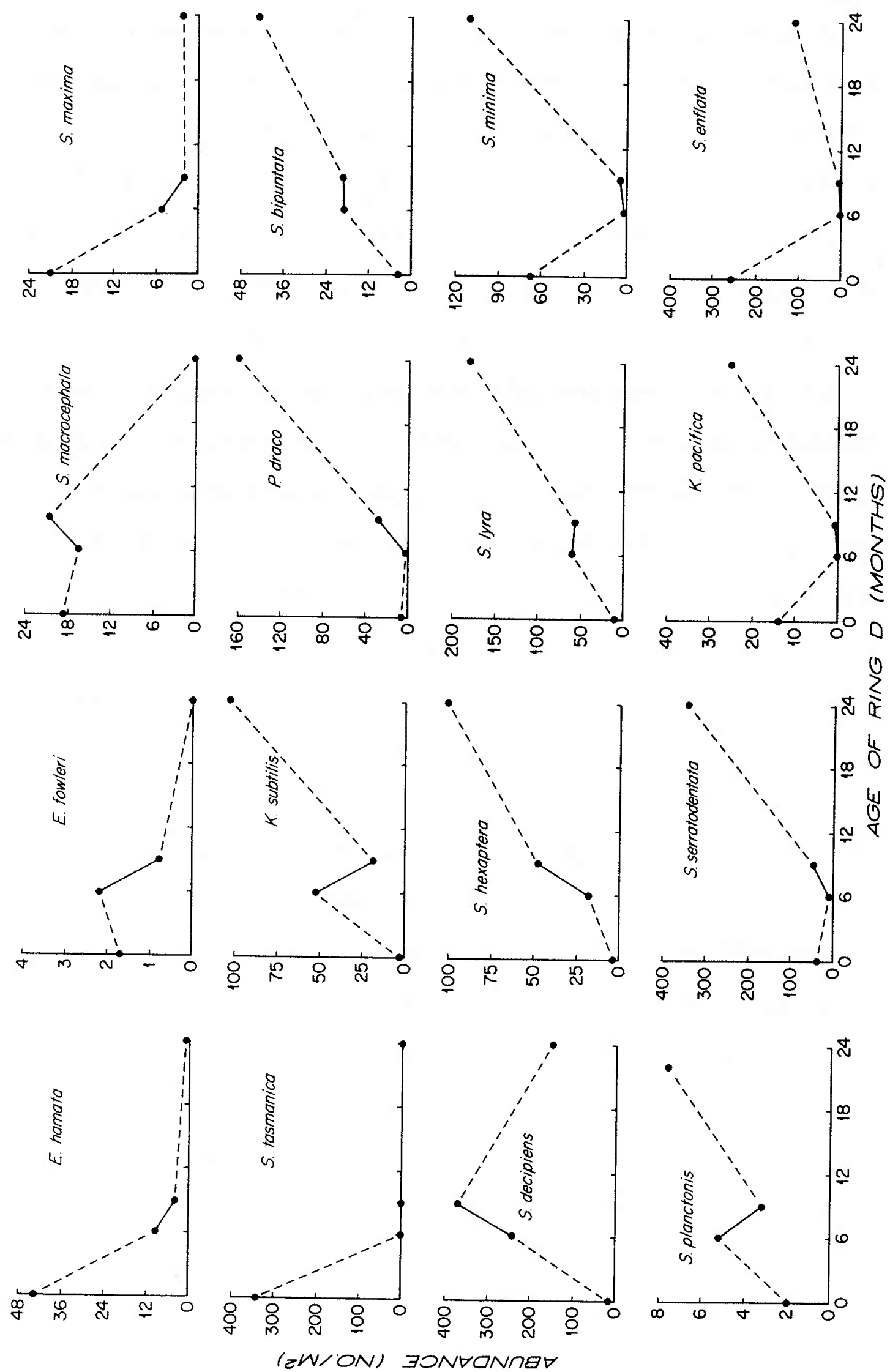

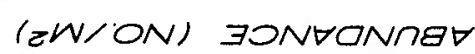


species, six increased in abundance from August to November in accord with this expectation, and three of the four SW species present decreased in abundance (Figure 5.6). Only four species showed significant differences in abundance between August and November [t-test, $\log _{10}(x+1)$ transformed abundances, $\mathrm{p}<0.05]$, however, and these were $\mathrm{k}$. subtilis, $\underline{\mathrm{P}}$. draco, S. hexaptera and S. 1yra. The observed abundance changes were in general agreement with the simple model discussed above.

Although the abundance data from Ring $D$ do not warrant detailed discussion because initial abundances were not known, the large numbers of

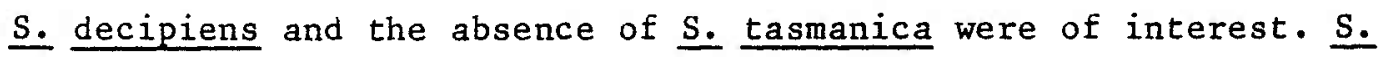
decipiens was notable for its relative lack of temporal variability in the NSS (Eigure 5.4), so its large numbers in Ring $D$ were probably due to real increases and not to sampling variability. Its large numbers suggest that some species may be able to exploit the unique nature of rings.

Assuming that initial abundances were the same and that the time course of changes in environmental conditions were similar in both rings, the presence of $\underline{S}$ tasmanica in Ring Bob after three months and its absence from Ring $D$ after six months indicates that environmental conditions altered sufficiently between three and six months to result in the extinction of the $S$. tasmanica population.

In general, the similarity of Ring Bob core stations with the SW and the relative lack of similarity of Ring $D$ core stations with the SW six months after formation provided evidence that significant environmental changes occur within the ring core between three and six months (Table 5.3). Further studies of cold core rings will be needed to characterize these environmental changes and their effects on biological populations 
Table 5.3. Percent similarity of Ring $D$ and Ring Bob cores with the SW and NSS. Ring core abundances were averaged for each ring and/or time, and SW and NSS abundances were averaged for the same cruise on which the ring was sampled. 
Ring D- August

0.129

0.475

Ring D- November

0.203

0.511

Ring Bob

0.720

0.219 
within rings.

Species association analysis

The recurrent group analysis produced only one large group containing sixteen of the twenty one species (Table 5.4). S. bipunctata and $\mathrm{S}$. helenae were associated with this group, while the three rarely collected

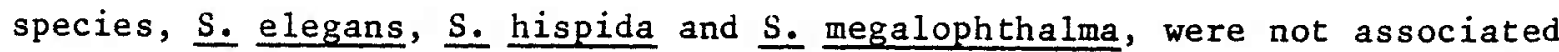
with any of the other species.

The results of the recurrent group analysis were in marked contrast with the species groupings from Table 5.1. Recurrent group analysis utilizes only presence/absence data to form groups and does not recognize quantitative abundance differences in the formation of these groups. While recurrent group analysis has been successfully applied to the study of the broad geographic distributions of plankton (Fager and McGowan, 1963; Venrick, 1971), its use in the analysis of spatial abundance patterns in restricted regions, especially where mixing across hydrographic boundaries is an important process, is not recommended.

CA and FA were applied to the total data set (52 MOCNESS tows and twenty one species) as well as to subsets of the data. I will limit discussion to the results of three of these analyses and a brief description of the data sets used in these three analyses will be presented before proceeding to the results.

FA 1 refers to the factor analysis applied to all twenty one species and only the SW and NSS tows. Only SW and NSS tows were used to provide results comparable with the species groupings from Table 5.1, which were 
Table 5.4. Results of the recurrent group analysis. Fractions in parentheses indicate the number of associations out of the total possible between the associated species and members of Group I. 


\begin{tabular}{|c|c|c|}
\hline Group I & Associated species & Unassociated species \\
\hline E. bathypelagica & S. bipunctata $(14 / 16)$ & S. elegans \\
\hline E. fowleri & S. helenae $(6 / 16)$ & S. hispida \\
\hline E. hamata & & S. megaloph thalma \\
\hline \multicolumn{3}{|l|}{ K. pacifica } \\
\hline K. subtilis & & . \\
\hline \multicolumn{3}{|l|}{ P. draco } \\
\hline \multicolumn{3}{|l|}{ S. decipiens } \\
\hline \multicolumn{3}{|l|}{ S. enflata } \\
\hline \multicolumn{3}{|l|}{ S. hexaptera } \\
\hline \multicolumn{3}{|l|}{ S. 1 yra } \\
\hline \multicolumn{3}{|l|}{ S. macrocephala } \\
\hline \multicolumn{3}{|l|}{ S. maxima } \\
\hline \multicolumn{3}{|l|}{ S. minima } \\
\hline \multicolumn{3}{|l|}{ S. planctonis } \\
\hline S. serratodentata & & \\
\hline S. tasmanica & & \\
\hline
\end{tabular}


based upon the species abundances in the SW and NSS. The $\log _{10}(X+1)$

transformed data proved to better delineate the species groupings than did the untransformed data, although the results of both analyses were quite similar. The better delineation of species groupings with the transformed data resulted from the improved correlations between species. Excluding al1 correlation coefficients for S. elegans, S. hispida, S. megalophthalma and S. helenae, each of which showed few significant correlations with the other species, the fraction of correlation coefficients significant at the 0.05 level was $88 / 136$ (65\%) for the untransformed data and was 104/136 (76\%) for the transformed data. The absolute magnitude of 111 of the 136 correlation coefficients increased after $\log _{10}(x+1)$ transformation.

Two of the correspondence analyses will be discussed. The first, CA 1 , for which only the species ordination will be presented, utilized the two GS stations as well as the SW and NSS stations. Results from other analyses showed that inclusion of the GS stations had negligible effects on the species factor scores. The three rarely collected species, $\underline{\mathrm{S}}$. elegans, S. hispida and S. megalophthalma, were excluded from this analysis, and again the results from other analyses showed that exclusion of these species had only minor effects on the species factor scores.

The second correspondence analysis, CA 2, for which only the station ordinations will be presented, included all 52 MOCNESS tows and the same eighteen species from CA 1. This analysis was chosen so that the relationships of the ring stations with the SW, NSS and GS stations could be observed.

One measure of the adequacy of these analyses is the percent of the total variability which is accounted for by each factor (Table 5.5). For 
Table 5.5. Percent variability accounted for by successive eigenvectors (factors). 


\begin{tabular}{llllllll}
\hline \multirow{2}{*}{ Factor } & \multicolumn{2}{c}{ CA 1} & \multicolumn{2}{c}{ CA 2} & \multicolumn{2}{c}{ FA 1} \\
& $\%$ & Cum. $\%$ & $\%$ & Cum. $\%$ & $\%$ & Cum. \% \\
\hline 1 & 49.8 & 49.8 & 43.6 & 43.6 & 50.9 & 50.9 \\
\hline 3 & 16.8 & 66.6 & 16.9 & 60.5 & 15.8 & 66.7 \\
4 & 9.6 & 76.2 & 10.9 & 71.5 & 7.4 & 74.0 \\
5 & 8.2 & 84.5 & 7.8 & 79.2 & 6.0 & 80.1 \\
6 & 4.2 & 88.7 & 5.9 & 85.1 & 5.2 & 85.2 \\
7 & 3.3 & 92.0 & 5.1 & 90.2 & 3.8 & 89.0 \\
8 & 3.0 & 95.0 & 2.5 & 92.7 & 3.0 & 92.0 \\
9 & 1.5 & 96.5 & 2.3 & 95.0 & 2.2 & 94.2 \\
10 & 1.2 & 97.7 & 1.8 & 96.8 & 1.3 & 95.5 \\
\hline & 1.1 & 98.8 & 1.3 & 98.1 & 1.1 & 96.5 \\
\hline
\end{tabular}


each of the three analyses, approximately half the variability was accounted for by the first factor, and about three-quarters of the variability was accounted for by the first three factors. As many factors can be extracted as there were species in the analysis, so the large fraction of the variability accounted for by the first three factors indicated that the three dimensional plots of species by their first three factor scores should have revealed most of the relationships in the data.

Another measure of the adequacy of the species ordinations is the percent of each species variability accounted for by each factor (Table 5.6). For $\mathrm{CA} 1$, eleven of eighteen species had $50 \%$ or greater of their variability accounted for by the first three factors. Sixteen of the same eighteen species in FA 1 had $50 \%$ or greater of their variability accounted for after three factors. It must be remembered that for FA this was the percent of the common variability (communality) which was accounted for. A value more comparable with the CA 1 results was obtained by multiplying the FA 1 cumulative percent value by the communality (Table 5.6). The resulting values were in general agreement with those from CA 1 . The communalities for FA 1 were generally large, with eighteen of the twenty one species having values greater than 0.60 .

Three dimensional plots of the species by their first three factor scores for both CA 1 and FA 1 (Figures 5.7 and 5.8 ) yielded species groupings similar to those obtained from the hydrographic abundance analysis presented in Table 5.1. For both ordination methods, the SW species from Table 5.1 had negative factor scores for the first factor, while NSS species had positive factor scores. Both $\underline{K}$. pacifica and $\underline{S}$. enflata had relatively low factor scores for the first factor in accord 
Table 5.6. Percent of each species variability accounted for after extraction of the first three factors and species communalities from factor analysis. 


\begin{tabular}{|c|c|c|c|c|c|}
\hline Species & $\begin{array}{l}\text { Abbreviation } \\
\text { for plots }\end{array}$ & CA $1 \%$ & FA $1 \%$ & Communality & $x F A \quad 1 \%$ \\
\hline E. bathypelagica & EBAT & 42.7 & 59.8 & 0.669 & 40.0 \\
\hline E. fowleri & EFOW & 47.1 & 67.2 & 0.699 & 47.0 \\
\hline E. hamata & EHAM & 66.3 & 90.1 & 0.916 & 82.5 \\
\hline K. pacifica & KPAC & 14.7 & 48.0 & 0.683 & 32.8 \\
\hline K. subtilis & KSUB & 72.0 & 92.9 & 0.932 & 86.6 \\
\hline P. draco & PDRA & 64.6 & 92.3 & 0.914 & 84.4 \\
\hline S. bipunctata & SBIP & 39.7 & 70.1 & 0.831 & 58.3 \\
\hline S. decipiens & SDEC & 69.9 & 86.0 & 0.932 & 80.2 \\
\hline S. elegans & SELE & - & 18.2 & 0.465 & 8.4 \\
\hline S. enflata & SENF & 93.5 & 81.3 & 0.688 & 55.9 \\
\hline S. helenae & SHEL & 48.3 & 85.9 & 0.701 & 85.9 \\
\hline S. hexaptera & SHEX & 89.1 & 94.5 & 0.947 & 89.5 \\
\hline S. hispida & SHIS & - & 60.5 & 0.701 & 42.4 \\
\hline S. 1yra & SLYR & 76.8 & 90.8 & 0.947 & 86.0 \\
\hline S. macrocephala & SMAC & 64.0 & 77.4 & 0.763 & 59.1 \\
\hline S. maxima & SMAX & 57.8 & 77.2 & 0.874 & 67.5 \\
\hline S. megalophthalma & SMEG & - & 5.8 & 0.302 & 1.7 \\
\hline S. minima & SMIN & 39.3 & 79.4 & 0.779 & 61.9 \\
\hline S. planctonis & SPLA & 28.6 & 34.8 & 0.568 & 19.8 \\
\hline S. serratodentata & SSER & 75.5 & 92.7 & 0.866 & $80 \cdot 3$ \\
\hline S. tasmanica & STAS & 97.4 & 89.8 & 0.913 & 82.0 \\
\hline
\end{tabular}


Figure 5.7. Three dimensional plot of species by their first three factor scores trom CA 1 . 


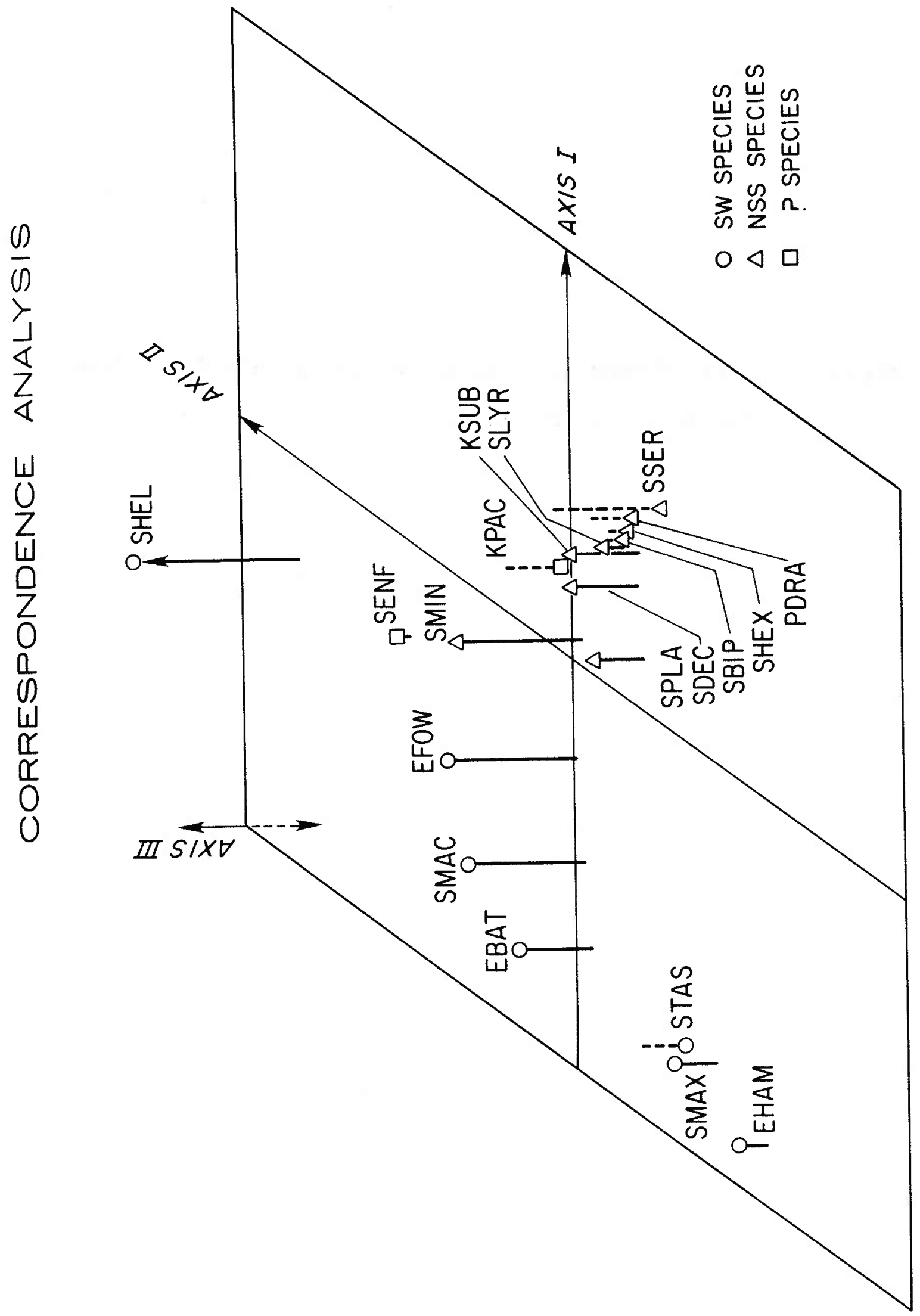


Figure 5.8. Three dimensional plot of species by their first three factor scores from FA 1 . 


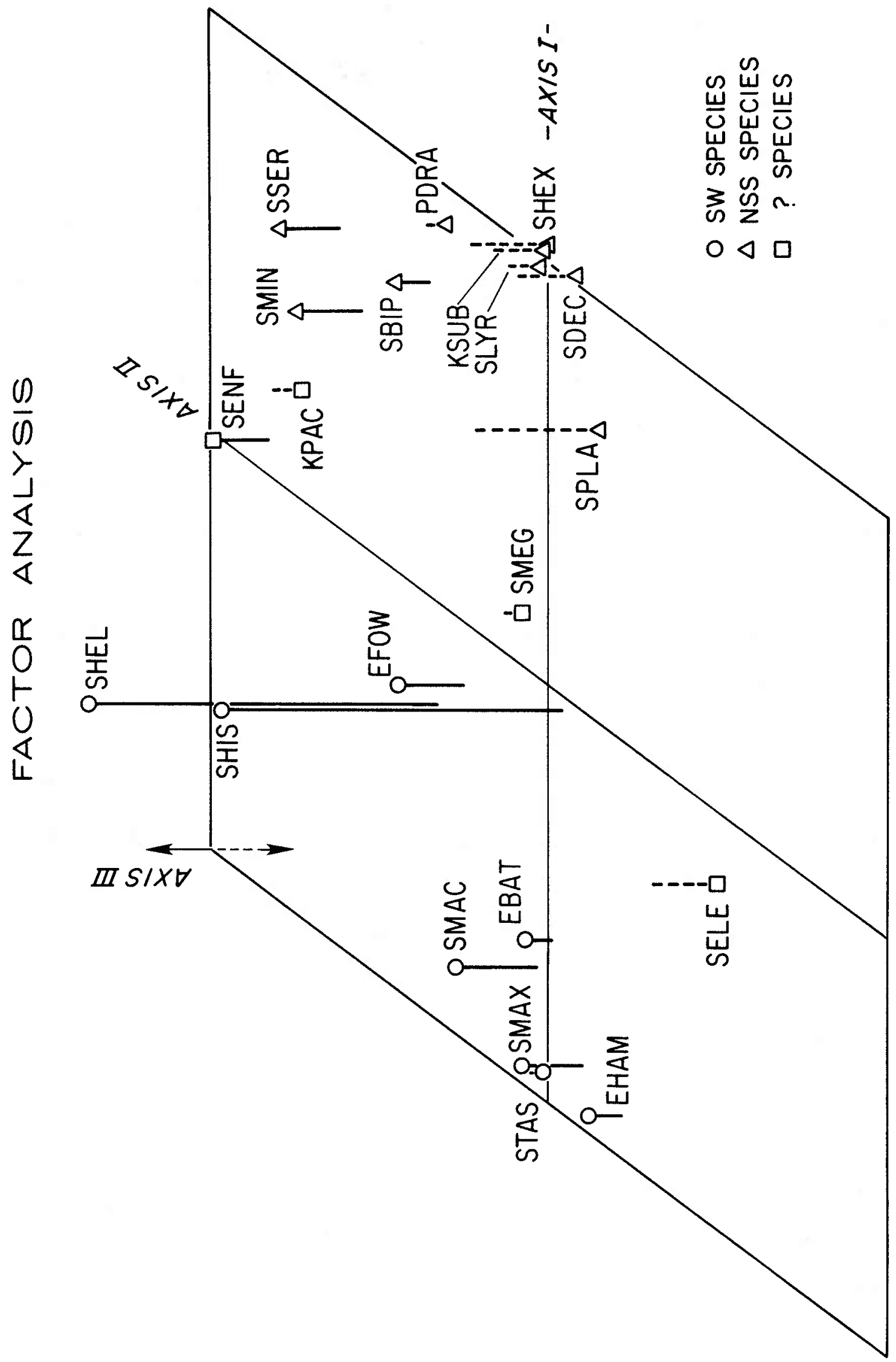


with their nonsignificant abundances differences between the SW and NSS. The three rarely collected species also had low first factor scores in FA 1.

Species factor scores for the first factor were related to their abundance differences between the SW and NSS. It the eighteen species from Table 5.1 are ranked according to the ratio of their NSS to SW abundance, the Spearman rank correlation coefficient for this ratio and the species first factor scores was 0.947 for $C A 1$ and was 0.932 for FA 1 .

Interpretation of the second and third factors were difficult because subsequent factors have been observed to be functions of previous factors (Gauch et al., 1977). This is especially true of FA after axis rotation. Subsequent factors tend to be dominated by one or two species, as indicated by the large third factor scores for S. helenae in CA 1 and $\underline{\text { S. }}$

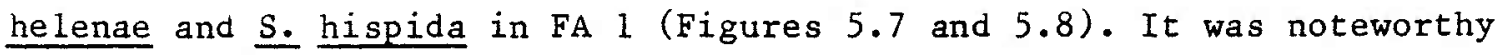
that both $\underline{S}$. helenae and $\underline{S}$. hispida had large third factor scores in FA 1 .

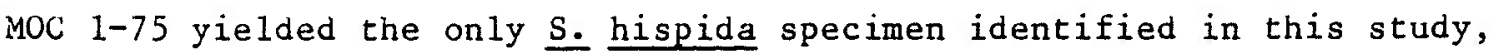
and the maximum abundance of $\underline{S}$. helenae was also recorded from this tow.

The station ordination from CA 2 also reflected the chaetognath abundance differences between the SW and NSS (Figure 5.9). The SW stations al1 had negative factor scores and the NSS stations all had positive factor scores for the first factor. The intermediate positions of the two GS stations were in agreement with their intermediate species abundances from Table 5.1. The five Ring Bob core stations were associated with the SW stations in accord with the percent similarity analysis discussed earlier. The four Ring Bob fringe stations represent the GS remnant, and they were associated with the NSS stations. Their similarity with the NSS 
Figure 5.9. Three dimensional plot of MOCNESS stations by their first three factor scores from CA 2. 
CORRESPONDENCE ANALYSIS

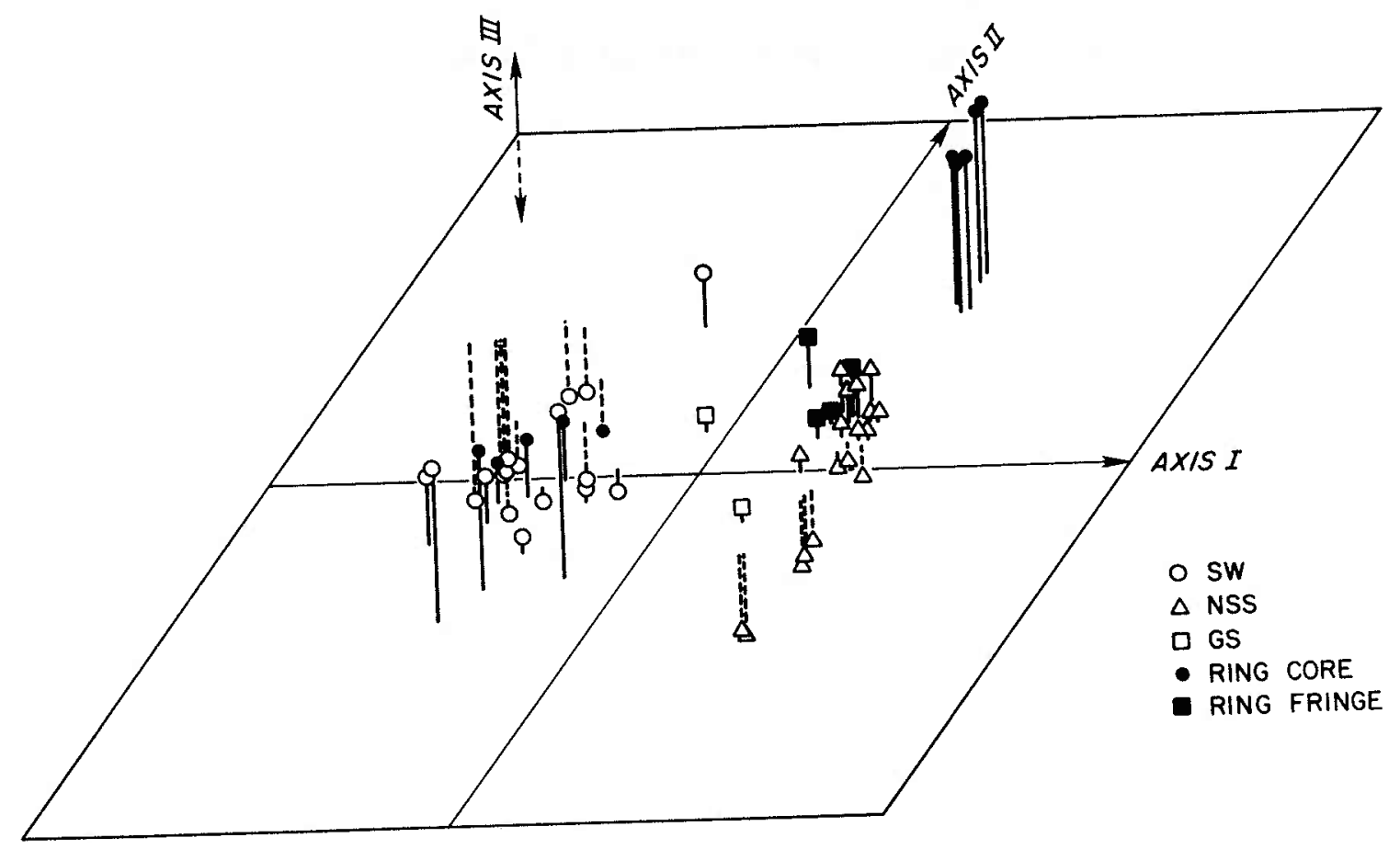


stations may have resulted from either an initial similarity of the GS and NSS during ring formation or the subsequent mixing of ring fringe with NSS water. The Ring D core stations from August and November of 1975 formed an isolated group of stations above the NSS stations (Figure 5.9). Their positive first factor scores indicated their relationships with the NSS stations, as was found in the percent similarity analysis, but their isolation from these stations along the second factor axis suggests that rings may represent unique biological environments which are not simply intermediate in character between the SW and NSS.

The results of the ordination analyses support the conclusions from the earlier analyses. This observation calls into question the usefulness of such methods as ordination analysis. In my view, ordination techniques are quite useful for preliminary data analysis. Relationships observed in the ordination analysis can be further studied using the original data. These techniques provide no more information than is contained within the original data, but they can considerably simplify the search for pattern within large, unwieldy data sets. The results of ordination analysis should not be used to supplant a careful analysis of the data, however.

Given that ordination is a useful procedure, which of the several methods available are most appropriate in given situations? CA and FA produced very similar species ordinations in the present study. The percent variability accounted for by each factor and the cumulative percent species variability accounted for by the first three factors were quite similar as discussed earlier. The product-moment correlation coefficients for the species factor scores from CA 1 and FA 1 were 0.98 for the first factor, 0.66 for the second factor, and 0.81 for the third 
factor. While there appears to be no basis for judging one technique better than the other based upon the species ordinations, CA is attractive in that both species and stations may be ordered simultaneously.

Determination of independent versus expatriated populations

Expatriated populations have rarely been identified in pelagic communities. McGowan (1960) and O'Day and Nafpaktitis (1967) identified expatriated populations of a pelagic worm and a midwater fish, respectively, on the basis of abnormal development of their reproductive organs. No obvious aberrations of the reproductive organs were observed for the chaetognaths in the present study. Expatriated populations may also be identified by the absence of particular developmental stages, especially the larval or juvenile stages. For S. 1yra, the only species from this study for which size frequency and sexual maturity were measured in all the samples, all sexual maturity stages were found in both the SW and NSS, although its SW size frequency distribution was shifted toward much larger individuals. Even though expatriated populations could not be identified by the above criteria, the low abundances for many of these species in the SW and/or NSS suggested that expatriated populations were present.

The following section represents an attempt to identify the presence of independent or expatriated populations based upon the abundances of these chaetognaths in the SW and NSS. The determination is partly subjective and partly objective, and where there is great doubt about the status of a population I have deferred judgement. The results of the following 
analyses are summarized in Table 5.7.

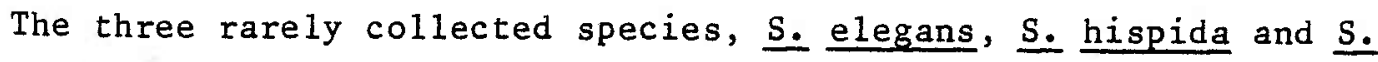
megalophthalma, were without doubt present in the SW/NSS region as expatriated populations. S. elegans is typically a boreal, coastal species, and it was reported by Grice and Hart (1962) to be the most abundant shelf water chaetognath in this region. They also reported it as the most abundant SW species, but a re-examination of their results showed that this species was abundant in the SW region only at their SW station adjacent to the she1f. It was found only in the MOC 1-116,117 collections, where the lowest surface salinity ( $32.70 \mathrm{ppt}$ ) was also recorded. These observations indicate that the presence of $\underline{\mathrm{S}}$ elegans in the SW region is limited to shelf water intrusions.

S. hispida is a tropical, coastal species in the Atlantic, and it is present as an independent population along the southeastern U.S. coast. Grant (1977) reported one battered specimen from $40^{\circ} 31.1^{\prime} \mathrm{N}$ and $70^{\circ}$ 53.8' $\mathrm{W}$ in June of 1963 . The one specimen collected in the present study was from the SW at $39^{\circ} 25^{\prime} \mathrm{N}$ and $69^{\circ} 32^{\prime} \mathrm{W}$. Owre (1960) reported S. hispida from the Florida Current off Miami, so it is likely that its presence in the SW is due to transport from the south by the Gulf Stream.

The distribution of $\underline{\mathrm{S}}$ megalophthalma is very poorly known. Dallot and Ducret (1969) described this species from collections in the Mediterranean and the Gulf of Guinea and Michel and Foyo (1976) reported it in small numbers from the Caribbean. It was found at one of the GS stations and one of the NSS stations in the present study. Its small numbers in the Caribbean suggest that it is also an expatriated population there. It is possible that this species is transported from the west African coast to 
Table 5.7. Presence of independent $(+)$ or expatriated (-) populations in the SW and NSS. 


$\frac{\text { Species }}{\text { Expatriated species }}$

S. elegans

S. hispida

S. megalophthalma

K. pacifica

S. helenae

Questionable species

E. bathypelagica

S. planctonis

E. fowleri

Slope Water species

E. hamata

S. maxima

S. tasmanica

Northern Sargasso Sea species

K. subtilis

P. draco

S. bipunctata

S. decipiens

S. hexaptera

S. 1yra

S. serratodentata

Species from both regions

S. entilata

S. minima

S. macrocephala
$+$

$+$

$+$

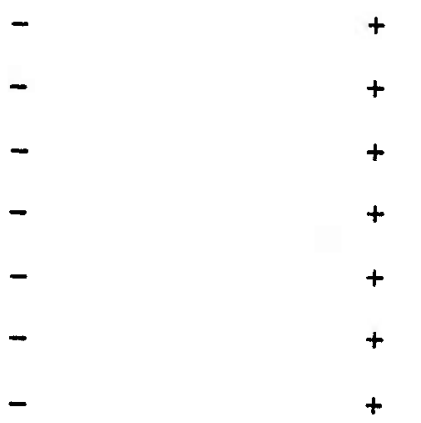

$+$

$+$
$+\quad+(?)$ $+$ $+$ $+$ $+$ 
the Caribbean by the North Equatorial Current, and from there it is transported to the SW/NSS region by the Gulf Stream. Whether $\underline{S}$. megalophthalma is present as an independent population along the west African coast or is an expatriate from the Mediterranean outflow is unclear.

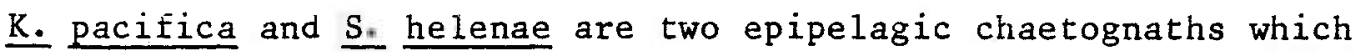
occurred in relatively small numbers and were thus presumed to be expatriates in the SW/NSS region. K. pacifica is an oceanic species found primarily in the equatorial regions of the world's oceans (Alvarino, 1965). It was found in the NSS infrequently and not at all in the SW by Grice and Hart (1962). Deevey (1971) reported K. pacifica as rare in the NSS off Bermuda and Moore (1949) did not report it from a study in the same region. Given its approximately equal abundances in the GS and NSS, I suggest that $\underline{K}$. pacifica is transported into the region from the Caribbean by the Gulf Stream.

S. helenae is a tropical, neritic species typical of the southeastern U.S. coast from Florida to Delaware (Alvarino, 1969; Grant, 1963). Alvarino (1969) reported $\underline{S}$. helenae from the shelf/slope region south of Cape Cod. Owre(1960) reported it from the Florida Current off Miami and it was present in approximately equal numbers in the $S W$ and $G S$ in the present study (Table 5.1), suggesting that it also is transported into the region by the Gulf Stream. This hypothesis is further supported by the occurrence of S. helenae in maximal numbers at the same SW station where the only $\underline{S}$. hispida specimen was found. The surface salinity at this station was 35.83 ppt, the fourth highest SW salinity observed in this study, thus suggesting that both these species were carried into the region by high 
salinity Gulf Stream water rather than low salinity shelf water intrusions. Removal of this station from the data results in a mean abundance of on 1 y 2.2 individuals $/ \mathrm{m}^{2}$ for $\mathrm{S}$. helenae in the SW as compared with 7.6 individuals $/ \mathrm{m}^{2}$ including the station.

Two other species, E. bathypelagica and S. planctonis, were present in low enough numbers to question their presence as independent populations. Alvarino (1969) reported E. bathypelagica only from the South Atlantic, but it has subsequent $1 \mathrm{y}$ been reported in small numbers from the Caribbean (Michel and Foyo, 1976), off the east coast of Canada (Figueira, 1972) and from the North Atlantic Current south of Greenland (Aurich, 1971). Given that this species is probably most abundant below $1000 \mathrm{~m}$ in the SW/NSS region (see Chapter 4 ), adequate assessment of its status in the region must await future studies of plankton abundance below $1000 \mathrm{~m}$.

The bimodal vertical distribution observed for $\underline{S}$. planctonis (Figure 4.2) suggested that two subspecies, S. planctonis planctonis and $\underline{S .}$. zetesios, were present in the study area. The abundance of both these forms combined was small relative to the other meso- and bathypelagic species (Table 5.1). Several investigators have recorded either one or both of these forms as uncommon in the North Atlantic (Grice and Hart, 1962; Moore, 1949; Michel and Foyo, 1976; Pierrot-Bults, 1969). Further studies of their abundances in other regions will be needed to determine whether or not they are present as independent populations in the SW/NSS region.

At face value, the low abundance of $\underline{E}$. fowleri made its presence as an independent population in the region questionable (Table 1). If we assume that the Eukrohnia spp. were juvenile E. fowleri, as discussed earlier, 
its SW abundance becomes 36.8 individuals $/ \mathrm{m}^{2}$ and its NSS abundance becomes 5.4 individuals $/ \mathrm{m}^{2}$. On this basis it may be argued that $\underline{\mathrm{E}}$ fowleri is present at least in the SW as an independent population. Further sampling to below $1000 \mathrm{~m}$ and a reliable means of identifying damaged and juvenile Eukrohnia specimens are needed for this species.

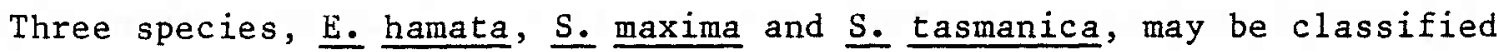
as having independent populations in the SW and expatriated populations in the NSS. The basis for this classification is a simple model first developed by Jahn (1976) in his analysis of expatriated midwater fish populations in the SW/NSS region. Earlier, it was determined that cold core rings were responsible for the addition to the NSS of a volume of SW equivalent to about two percent of the volume of the upper $1000 \mathrm{~m}$ of the NSS. Any species present in the NSS in less than two percent of its SW abundance may thus be presumed to have been brought into the region by cold core rings. E. hamata abundance in the NSS was $2.0 \%$ of its SW abundance, and S. tasmanica abundance in the NSS was $0.09 \%$ of its SW abundance. S. maxima abundance in the NSS was $9.5 \%$ of its SW abundance, but an examination of the data showed that this relatively large percentage was due to its presence in large numbers in the MOC 1-64,65 collections. It these two tows are excluded from the data, S. maxima abundance in the NSS becomes 0.25 individuals $/ \mathrm{m}^{2}$ or $1.2 \%$ of its SW abundance. It must then be presumed that the large numbers in the MOC $1-64,65$ collections were due to an anomalous transport of this species from the SW into the NSS. One would expect to see large numbers of the associated SW bathypelagic species, E. bathypelagica, E. fowleri, E. $\underline{\text { hamata }}$ and $\underline{\text { S. macrocephala, }}$, at these stations if such a transport 
occurred, but anomalously large abundances were not observed for these species. The large numbers of $\underline{\mathrm{S}}$. maxima at these two stations thus remain

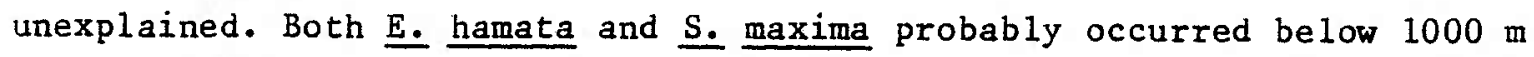
in the NSS, so further sampling studies are needed to confirm their classifications as expatriates in the NSS.

The model used in this analysis has several shortcomings. Variability in the sizes of rings and their frequency of formation may alter the volume of SW transported to the NSS. It also assumes that ring water is instantly mixed with NSS water, but this is clearly not the case. Thus, while a two percent addition to the NSS occurs, the SW core and its plankton populations remain isolated from the NSS in rings. There may also be other mechanisms by which SW populations are added to the NSS to increase the abundance of SW species in the NSS, such as transport by the Western Boundary Undercurrent. The model does represent a usetul approximation, however, and it may be used with other information for the assessment of the status of populations within the region. The relative lack of E. hamata and $\underline{S}$. maxima juveniles in the upper $1000 \mathrm{~m}$ of the NSS supports the hypothesis that they are expatriates in the NSS.

Using the same simple model, it is possible to identify species with independent WSS populations and expatriated SW populations. Earlier it was determined that warm core rings add twelve percent to the volume of the SW in the form of Gulf Stream and NSS water. Assuming that GS abundances were equal to NSS abundances, species whose SW abundances were less than twelve percent of their NSS abundances were $\underline{\mathrm{K}}$. subtilis $(3.1 \%), \underline{\mathrm{P}}$ draco $(3.2$ $\%)$, S. bipunctata $(8.6 \%)$, s. decipiens $(9.8 \%)$, S. hexaptera $(3.2 \%)$, s. lyra $(5.0 \%)$ and S. serratodentata $(10.7 \%)$. Each of these species is a 
typical inhabitant of warm and temperate oceanic environments and, with the exception of S. serratodentata, which is restricted to the Atlantic Ocean, they are cosmopolitan in the world's oceans (Alvarino, 1965).

Knowledge of the hydrography of the SW region may be used to develop another simple test of presence of the above seven species as expatriates in the SW. Since the upper $200 \mathrm{~m}$ of the $\mathrm{SW}$ is a mixture of shelf water and GS/NSS water, the surface salinity may be used as a measure of the quantities of shelf water and GS/NSS water used in its formation. Assuming a shelf water salinity of $34.0 \mathrm{ppt}$ and a GS/NSS salinity of $36.0 \mathrm{ppt}$, we may calculate the quantities of any conservative property given SW surface salinity and quantities present in the shelf and GS/NSS waters. Biological populations are not conservative properties, so if these species can survive and reproduce in the SW then their abundances should be greater than that predicted by the mixing curve, but if only mortality occurs in the populations, then we expect lower abundances than predicted by the mixing curve. Assuming their abundances in the shelf water were zero, the seven species discussed above were generally less abundant than predicted from the mixing curves (Figure 5.10). It may be concluded that these species are expatriates in the SW. This model is relatively robust in that either NSS or GS abundance may be used as the initial GS/NSS abundance, and shelf water salinity may be varied from 32 to 35 ppt with little effect on the conclusions reached.

$\underline{\text { S. enflata, }}$ S. minima and $\underline{S}$. macrocephala can be assigned independent population status in both the SW and NSS by virtue of their relatively large abundances in both regions. Although the NSS abundance of $\underline{S}$. macrocephala was about five times less than its SW abundance (Table 5.1), 
Figure 5.10. Species abundance as a function of surface salinity in the SW. The solid line represents the mixing curve using NSS abundance (Table 5.1) at 36 ppt and dashed line represents mixing curve using GS abundance (Table 5.1) at 36 ppt. 

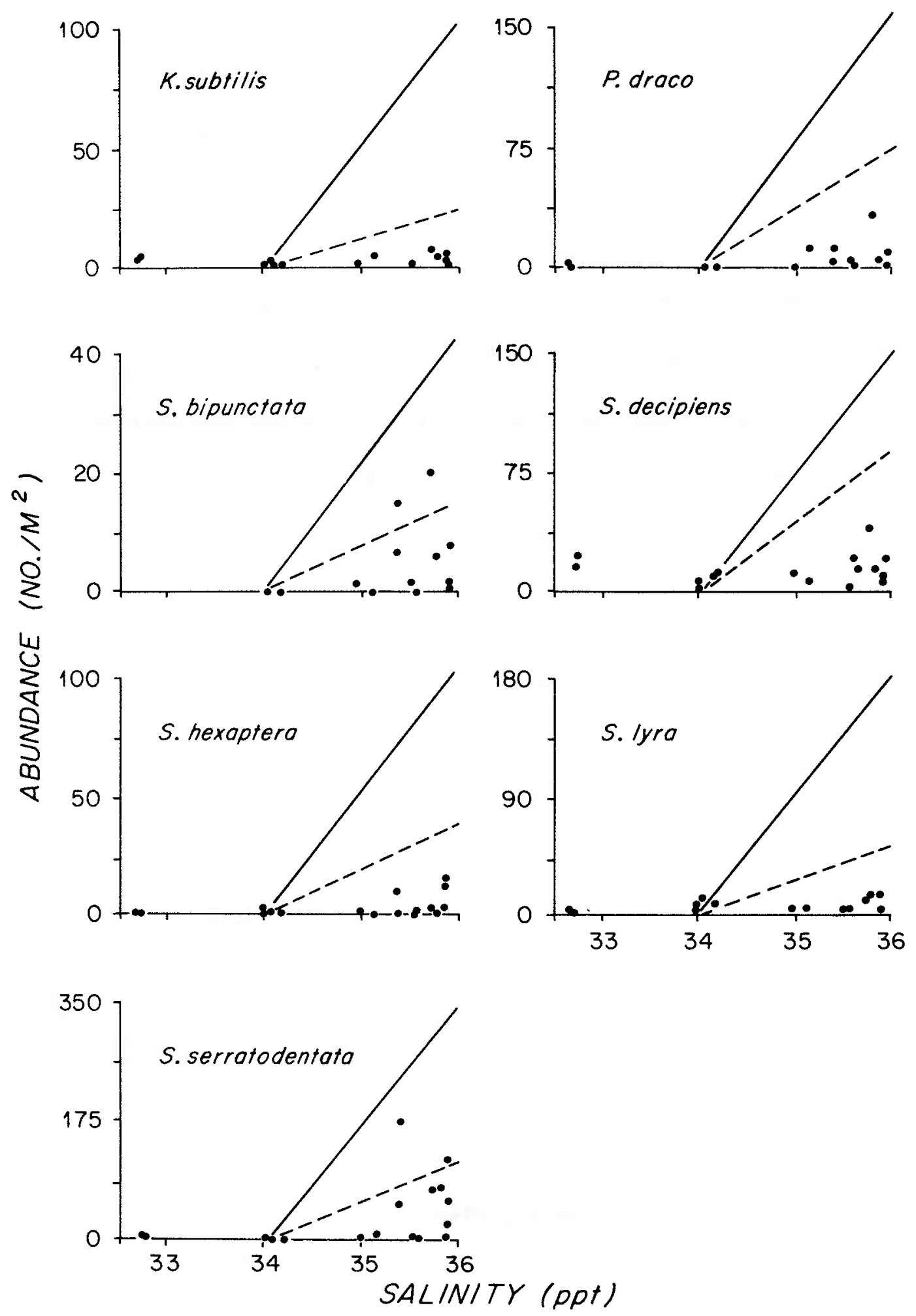
it was well above the two percent SW abundance expected of expatriated

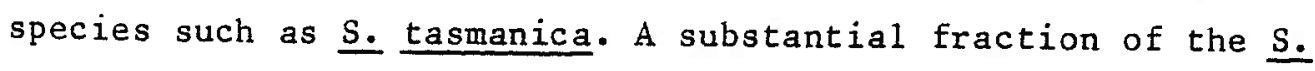
macrocephala population was undoubtedly present below $1000 \mathrm{~m}$ in both the SW and NSS (Figure 4.2), so further sampling studies are needed.

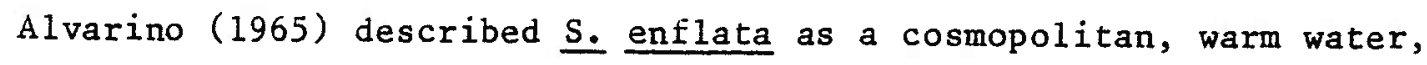
oceanic species, but reports from the western North Atlantic indicate that it is most abundant in coastal waters. Several studies have characterized S. enflata as the most abundant chaetognath in continental shelf waters south of Cape Hatteras (Pierce, 1953; Bumpus and Pierce, 1955; Pierce and Wass, 1962), and it has been recorded frequently from shelf waters north of Cape Hatteras as we11 (Bigelow and Sears, 1939; Grice and Hart, 1962; Grant, 1977). Grice and Hart reported it as abundant in both the $\mathrm{SW}$ and NSS, but Moore (1949) and Deevey (1971) found it only rarely in the NSS off Bermuda. Although not significantly different from its NSS abundance, its greater abundance in the SW in the present study was consistent with other observations, since SW conditions more closely resemble those of coastal regions than do those of the NSS. S. enflata thus appears to be primarily a coastal species in the western North Atlantic, with limited abilities to maintain independent populations in oceanic environments.

S. minima is also described as a cosmopolitan, warm water, oceanic chaetognath by Alvarino (1965), but Michel and Foyo (1976) considered it to be most abundant in oceanic waters near the continental shelf. Bieri (1959) reported S. minima as inhabiting regions between water masses in the Pacific. Grice and Hart (1962) found this species infrequently in the SW and regularly in the NSS, and Deevey (1971) reported it as the most abundant species in the NSS off Bermuda. In the present study, S. minima 
was approximately twice as abundant in the NSS as in the SW (Table 5.1). The geographic abundance pattern for S. minima in the western North Atlantic appears to be opposite that for $\underline{S}$ enflata. That is, moving from a coastal to an oceanic environment results in an increase in $\mathrm{s}$. minima abundance and a decrease in S. enflata abundance. I must agree with Michel and Foyo (1976) in that the distribution of S. minima is poorly known in the Atlantic due to inadequate sampling because of its small size at maturity (8 $\mathrm{mm}$ in length).

\section{Discussion}

Comparisons with previous studies

The chaetognath species composition found in the present study corresponds well with the results irom previous studies in the region (Moore, 1949; Grice and Hart, 1962; Alvarino, 1969; Deevey, 1971). Grice and Hart (1962) studied the zooplankton distributions from the upper $200 \mathrm{~m}$ along a transect from New York to Bermuda, Alvarino (1969) summarized the geographic distributions of Alantic chaetognaths from published reports and her own data, and Moore (1949) and Deevey (1971) studied the zooplankton of the upper $500 \mathrm{~m}$ off Bermuda. S. megalophthalma and E. bathypelagica were the only two species not previously reported from the SW/NSS region. The few individuals of $\mathrm{S}$. megalophthalma transported into the region by the Gulf Stream are of no consequence ecologically, and previous records of E. bathypelagica to the north (Figueira, 1972; Aurich, 
1971) and to the south (Michel and Foyo, 1976) suggested that it would be found in the SW/NSS region also.

Grice and Hart (1962) did not report S. tasmanica from the SW, but it was clearly the most abundant SW species in the present study. Grant (1967) re-examined their specimens of $\underline{S}$. serratodentata from the SW and found them to be primarily S. tasmanica. Since Grice and Hart sampled only the upper $200 \mathrm{~m}$, their report did not include several of the bathypelagic species in the region. Their identification of $\mathrm{s}$. ferox in small numbers and Moore's (1949) identification of $\mathrm{s}$. robusta in small numbers in the

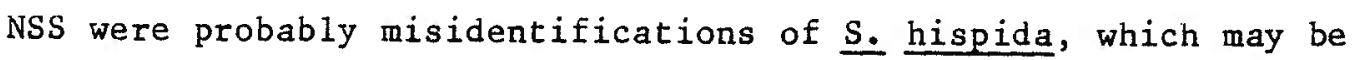
transported into the region by the Guli Stream or from Bermuda coastal waters. Grice and Hart also recorded $\underline{\text { S. friderici }}$ as rare in the NSS, and McLelland's (1980). report of its presence in the Gulf of Mexico suggests that it too is carried northward by the Gulf stream.

Grice and Hart (1962) and Deevey (1971) provided abundance estimates which may be compared with those of the present study. Although Grice and Hart's report of $\underline{S}$ elegans as the dominant $S W$ species was in error as discussed earlier, their repots of the two remaining abundant SW species, S. entiata and S. tasmanica (their S. serratodentata), and seven relatively rare species, $\underline{\text { P. draco, }} \underline{\text { S. bipunctata, }}$ S. decipiens, $\underline{\text {. }}$ hexaptera, S. lyra, S. minima and S. planctonis, correspond well with the results of the present study. Grice and Hart suggested that the presence of the latter seven species was related to the mixing of Gulf stream and Slope Waters, a result confirmed here for all but $\underline{S}$. minima and $\underline{S}$. planctonis. They reported S. serratodentata as the most abundant NSS species, as was also the case in the present study. 
Abundances estimated for eight NSS species from Figure 13 of Deevey (1971) were generally within a factor of three of those presented in Table 5.1. The only striking differences were that $\underline{\mathrm{s}}$ minima was the most abundant species and $\underline{S}$ enflata was only rarely collected off Bermuda. That Deevey found an average of more than 800 indiviuals $/ \mathrm{m}^{2}$ as compared with 110 individuals $/ \mathrm{m}^{2}$ for $\mathrm{S}$. minima from Table 5.1 may be due to year to year variations in abundance, geographical abundance differences within the NSS, or to Deevey's use of a net with smaller mesh size than was used in the present study. The difference in $\underline{S}$ enflata abundance was discussed earlier (see Results). Spring abundance peaks were evident from Deevey's data for S. minima, S. serratodentata, S. hexaptera and S. 1yra, but not for $\underline{\text { K. subtilis, }}$ S. decipiens, $\underline{\text { P. draco }}$ or S. bipunctata.

That the abundances found in these three studies are comparable strongly argues that net sampling provides abundance estimates which are representative of larger time and space scales of zooplankton abundance. Previous studies of the geographic distributions of plankton have relied primarily on presence/absence data (although the work of Bieri (1959) on Pacific chaetognaths provides a remarkable exception). If only presence/absence data had been reported from this study, the relationships of these species with the conditions necessary to support independent populations would have been greatly obscured. Beklemishev (1976) argued that frequency of capture in different geographic locations should be used to construct charts of the geographic distributions of zooplankters, but this is only a crude approximation to abundance. We now know enough about the errors associated with net sampling to design appropriate sampling techniques for estimating the abundances of these plankters, and it is 
argued here that future studies of the geographic distributions of plankters should be directed toward collecting abundance data rather presence/absence data.

The importance of rings for the study of plankton distributions

Mesoscale rings and eddies are known to form in a number of regions, and they have significance for the study of plankton geography for several reasons.

Rings, if unrecognized, may obscure the relationships of geographic distributions and hydrography. Bieri (1959) did recognize anomalous distributions of chaetognaths in the North Pacific which probably resulted from both warm and cold core ring formation by the Kuroshio Current, as he wrote,

"The occurrence of $\underline{\mathrm{S}}$ elegans at Transpacific Station $112 \ldots$, considerably south of its usual southern limit, is accompanied by an absence of many warm-water species... The fauna at this station is interpreted as an isolated community that was carried considerably south of its usual range. The same phenomenon is shown at Transpacific Station $60 \ldots$, where a segment of the warm-water fauna has been carried north of its usual range...."

In addition to understanding the relationships of independent populations with environmental conditions, we are interested in understanding the mechanisms which produce expatriated populations. 
Because rings are consistent features in regions of western boundary currents, they may be largely responsible for the formation of expatriated populations in those regions.

Rings may also be useful as large scale experimental sites for the study of the processes regulating geographic abundance patterns (Wiebe et al., 1976b). Because physical and biological properties of hydrographic regions are correlated, it has been difficult to isolate the important factors regulating geographic patterns. Differences in the rates of change of biological and physical properties in rings may allow the separation of the important processes.

An example of the use of rings in the study of geographic distributions was provided by Wiebe and Boyd (1978) and Boyd et al. (1978). They studied the distribution of the euphausiid, Nematoscelis megalops, in the SW/NSS region, and found it to be almost exclusively associated with the SW. Its presence within the NSS was limited to cold core rings or could be traced to the recent passage of a ring. Observations within rings showed that $\mathrm{N}$. megalops abundance decreased with time until extinction of the population occurred. Its vertical distribution deepened with time, apparently following the depth of the $10^{\circ} \mathrm{C}$ isotherm. Biochemical and physiological measurements made on ring and $\mathrm{SW}$ individuals suggested that ring populations were slowly starving. It may be hypothesized that their selection of a vertical distribution around the $10^{\circ} \mathrm{C}$ isotherm removed them from food rich upper waters and resulted in their eventual starvation. Wiebe and Boyd (1978) argued that this was the likely mechanism for the restriction of N. megalops to the SW.

Further studies in both warm and cold core rings in the western North 
Atlantic should provide much needed information for assessing the mechanisms which regulate the geographic distributions of plankton.

Regulation of the geographic and seasonal abundance patterns

Understanding the regulation of the spatial and temporal abundance patterns of organisms is primarily a problem of understanding their population dynamics under changing environmental conditions. Any factor which affects an organism's birth or death rates may therefore be important in regulating its abundance over space and time. Environmental conditions in the oceans change rapidly with depth, so we must understand how zooplankters choose the depths at which they live as well as the resultant effects of these environmental conditions on birth and death rates.

While it will be necessary to test the effects of changes of environmental conditions on birth and death rates in controlled laboratory conditions, field sampling studies can provide insight into the important factors regulating the observed abundance patterns. If expatriated populations can be distinguished from independent populations, then conditions where independent populations are found may be compared with conditions where they are not found, and testable hypotheses may be deve loped.

The importance of several processes regulating chaetognath abundance patterns in the western North Atlantic will now be discussed. The limited number of factors to be discussed were chosen because information was available regarding their spatial and temporal variations in the region, 
and because they are known to affect birth and death rates. Because conditions in regions with independent and expatriated populations must be compared, only those species with independent populations in either the SW or NSS and expatriated populations in the other region (see Table 5.7) will be discussed.

Processes responsible for the horizontal abundance patterns will be discussed first, followed by a brief discussion of those processes responsible for the spring peak in chaetognath abundance in the NSS. Birth rates are a function of growth rates and subsequent egg production, so each factor will be considered in relation to its effect on growth rate, egg production and mortality before consideration of its role in limiting the geographic distributions of chaetognaths in this region.

\section{A. Food}

Although little direct evidence is available, food abundance must play a major role in the growth, reproduction and survival of chaetognaths. Reeve (1970) showed that growth rate of S. hipida in the laboratory depended upon food concentration. The number of animals with mature eggs was also found to increase with increasing food levels, and survival was higher at the larger food concentrations. Reeve (1963), Feigenbaum and Reeve (1977) and Reeve (1981) showed that ingestion rate for several chaetognaths increased with increasing food abundance up to a maximal feeding rate. Reeve (1981) discussed a possible lower feeding threshold for $\underline{S}$. elegans, S. enflata and $\underline{S}$. hispida in relation to environmental food levels, which were generally observed to be below the threshold feeding level. He argued that chaetognaths must depend upon patchy food distributions to obtain adequate food. These studies indicate that food 
concentrations in the environment can limit ingestion rates of chaetognaths, and can therefore limit their growth and reproduction and increase their mortality.

Numerous studies of the gut contents of chaetognaths indicate that copepods are the primary prey, but a wide variety of other plankters may be taken as wel1 (Lebour, 1922, 1923; Nagasawa and Marumo, 1972, 1976; Rakusa-Suszczewski, 1969; Stone, 1969; Mironov, 1960; Szyper, 1978; Pearre, 1973, 1974, 1976; Sullivan, 1980). These studies have shown that food selection, as indicated by differing proportions of food in their guts and the environment, is primarily based upon prey size differences, but there is evidence that other mechanisms may be involved (Pearre, 1974; Sullivan, 1980). Pearre (1974) suggested that prey avoidance capabilities may be responsible for some of the selectivity observed. Horridge and Boulton (1967) first showed that the benthic chaetognath, Spadella cephaloptera, detected prey by mechanoreception and responded to a vibrating probe over a narrow frequency range from 9 to 20 cycles/second. Newbury (1972) speculated that such a narrow frequency response might lead to prey selection because different copepod species produce vibrations of their feeding appendages at different frequencies. It was subequently shown by Feigenbaum and Reeve (1977) that Sp. cephaloptera and two other species, Sp. schizoptera and $\underline{\mathrm{S}}$. hispida, responded to a much wider range of frequencies than Newbury reported for copepods. Experimentally, Reeve (1966) and Reeve and Walter (1972) found selection of food by size for $\underline{\text { S. }}$ hispida. For the moment, selection by size is the only mechanism of chaetognath prey selection with firm observational and experimental support. 
As discussed above, experimental work has shown that some chaetognaths detect prey by mechanoreception, and they will only feed upon live, active prey (Horridge and Boulton, 1967; Reeve, 1966; Feigenbaum and Reeve, 1977). Because chaetognaths are a morphologically similar group, it may be presumed that a11 species detect prey by mechanoreception.

The above information indicates that that the availability of active prey of the appropriate size can limit the abundance of chaetognaths in nature. Rakusa-Suszczewski (1969) argued that the size distribution of available prey altered both the abundance and size distribution of $\underline{\mathrm{S}}$. elegans in parts of the North Sea.

Detalled investigation of the hypothesis of food limitation requires knowledge of the prey selection by individual species and the distribution of prey in nature. Since neither of these is known in detail for the SW/NSS region, zooplankton biomass will be used as an approximate indicator of prey abundance. Grice and Hart (1962) found that copepods averaged $66 \%$ (excluding stations dominated by salps) and $48 \%$ of the zooplankton biomass in the upper $200 \mathrm{~m}$ of the SW and NSS, respectively. Ortner et a1. (1978) reported that SW zooplankton biomass in the upper 750 m averaged 3.5 times greater than in the NSS, and Grice and Hart (1962) reported a SW/NSS zooplankton biomass ratio of 4.0 in the upper $200 \mathrm{~m}$, as did Clarke (1940). Utilizing only the 1976-1977 tows from the present study, The SW/NSS biomass ratio in the upper $1000 \mathrm{~m}$ was 2.0 . In general, zooplankton biomass decreases with depth in both these regions, and at any given depth in the SW, zooplankton biomass is greater than NSS biomass. On the basis of total food availability (measured as zooplankton biomass), it cannot be argued that food limitation excludes NSS species 
from the SW. It may be argued, however, that NSS species are excluded by a lack of food of the appropriate size. Over a wide range of chaetognath sizes, prey size is generally related directly with chaetognath size, although there may be some differences in the exact nature of this relationship between species (Pearre, 1980) Assuming that all chaetognaths hatch as $1.0 \mathrm{~mm}$ larvae (Table 3.9 ), the size ranges for the SW and NSS species overlaps considerably (see Table 3.7 for maximum lengths for these species). This suggests that prey sizes must also be similar in the SW and NSS in order to support chaetognath populations of similar sizes, thus arguing against prey size differences as a mechanism for the exclusion of NSS species from the SW.

Food limitation is, however, a viable hypothesis for the exclusion of the SW species, E. hamata, S. maxima and S. tasmanica, from the NSS. If these species occupy the same depths in both regions, there will be approximately two to four times less food available in the NSS. If they orient their vertical distributions to temperature, they will occupy greater depths in the NSS and thus be even further removed from an abundant food supply. This is essentially the mechanism proposed by Wiebe and Boyd (1978) for the exclusion of N. megalops from the NSS. Neither of these means of selecting their vertical distributions could be rejected for these species from the analysis in Chapter 4.

\section{B. Temperature}

Temperature has long been considered one of the most important factors limiting the geographic distributions of zooplankton. Temperature affects most, if not all, physiological processes, with subsequent effects on the growth, egg production and mortality. At the extremes of the temperature 
range tolerated by an organism, mortality will increase and thus limit its abundance. Within the range of temperature tolerated, however, temperature effects on growth and egg production may result in the exclusion of a species from a geographic region. Decreasing temperature results in a decreased growth rate for the chaetognath S. hispida (Reeve, 1970; Reeve and Walter, 1972; Reeve and Baker, 1975). Owre (1960) suggested a relationship between latitude and generation time for chaetognaths from field studies, and this is presumably caused by decreased growth rates at lower temperatures. Slower growth rates may result from decreased ingestion rates at lower temperature (Reeve, 1966) due to increased digestion times at lower temperatures (Pearre, 1981). Associated with slower growth rates at lower temperatures is increased body size at maturity (Russe11, 1932; McLaren, 1966; Reeve and Walter, 1972), which would further delay the onset of maturity and thus further decrease the

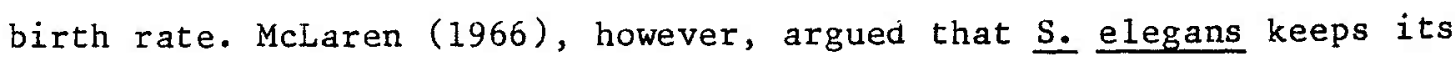
birth rate relatively constant by producing more eggs as body size at maturity increases. Reeve (1970) showed that egg production by $\underline{S}$. hispida was directly related with temperature, thus arguing against McLaren's observations, at least for $\underline{\mathrm{S}}$. hispida. Further work is needed to determine the relative effects of temperature on growth rate and egg production for other chaetognaths.

Seasonal temperature fluctuation is much greater in the $\mathrm{SW}$ than the NSS (Figure 5.2). In the $\mathrm{SW}$, surface temperatures may range from about $8^{\circ} \mathrm{C}$ in late winter to $27^{\circ} \mathrm{C}$ in late sumer. In the winter, there is generally a subsurface (50-150 m) temperature maximum which rarely falls below $10^{\circ} \mathrm{C}$ ( $\mathrm{P}$. Wiebe, unpublished data). There is little variation in 
temperature below $200 \mathrm{~m}$ (Figure 4.1). In the NSS, surface temperature ranges from $27^{\circ} \mathrm{C}$ in the summer to a $17^{\circ} \mathrm{C}$ minimum in the spring, and from the surface to a depth of about $500 \mathrm{~m}$, temperature is never below $17^{\circ} \mathrm{C}$

Temperature is a likely factor excluding the seven NSS species from the SW. P. draco, S. bipunctata and S. serratodentata were confined to the upper $200 \mathrm{~m}$ in both regions, and S. hexaptera, S. decipiens, S. 1yra and K. subtilis were primarily confined to the upper $500 \mathrm{~m}$ in both regions. Thus during the late winter in the SW, these species would be exposed to temperatures at least $7^{\circ} \mathrm{C}$ lower than they experience in the NSS. There is, however, not enough information to assess the temperature tolerances of any of these species.

Temperature is also a possible factor limiting E. hamata, S. maxima and S. tasmanica from the NSS. If these species orient their vertical distributions to temperature, then the hypothesis of temperature limitation must be discarded, but as discussed in Chapter 4, there are alternative interpretations of their vertical distributions. If these species orient their vertical distributions to light or pressure, they will be found at approximately the same depths in the SW and NSS. S. tasmanica was found primarily in the upper $200 \mathrm{~m}$ of the $\mathrm{SW}$, but personal observations made while SCUBA diving in the North Atlantic showed that it lives only within and below the seasonal thermocline, and thus it is probably not exposed to the high SW temperatures in late summer. Because of the large sampling intervals (50 to $100 \mathrm{~m}$ ) in the upper $100 \mathrm{~m}$ during the present study, it was not possible to confirm or refute the observation that $\underline{\text { s. tasmanica }}$ lives only in or below the seasonal 
thermocline in the SW. It was clear from the MOCNESS samples that a substantial proportion of its population lived in waters of less than $13^{\circ} \mathrm{C}$ below the seasonal thermocline. Water of this temperature is found in the NSS at a depth of about $800 \mathrm{~m}$. It appears that in the upper $200 \mathrm{~m}$ of the NSS, S. tasmanica probably is exposed to considerably higher temperatures than in the SW. If this were the case, the few individuals found at deptin at in the NSS (Figure 4.2) may represent dead or dying individuals sinking out of the surface waters.

S. maxima and E. hamata populations were found principally below $200 \mathrm{~m}$ in the SW (Figure 4.2), where temperature at any given depth would be 5 to $10^{\circ} \mathrm{C}$ warmer in the NSS (Figure 4.1). High temperature and/or low food may then be excluding these species from the SW, depending upon how they select their vertical distributions.

C. Predation

Predation is undoubtedly one of the most important sources of mortality for zooplankters. Field studies of predation mortality are not easy to perform because of the difficulty of identifying all the predators on a given species, so it has generally been assumed in theoretical studies that predation is related to predator abundance and prey abundance (Steele, 1974b). Increased predator abundance leads to increased prey mortality, and differences in predator abundance between the SW and ivSS may exclude chaetognaths from one of the regions.

It is known that midwater Eish, decapod crustaceans, euphausiids, copepods, medusae, siphonophores and chaetognaths are predators on chaetognaths (Merrett and Roe, 1974; Donaldson, 1975; Mauchline and Fisher, 1969; Omori, 1974; Stone, 1969). It is not known, however, what 
species are predators on chaetognaths in the SW/NSS region. Many of the predators are probably included in the measurement of zooplankton biomass, but without a knowledge of the proportion of predators in the zooplankton biomass, it is unwise to use zooplankton biomass as a measure of predator abundance. Samples collected with the MOC 10 trawI (see Methods) contain primarily large, predaceous animals such as midwater fish, decapod crustaceans, medusae and siphonophores. Although certainly not a perfect measure of predator abundance, the biomass from the MOC 10 collections may provide a measure of the relative abundance of predators in the SW and NSS. Biomass of both the invertebrate and vertebrate (fish) components of the MOC 10 collections from Knorr 65 and Knorr 71 were kindly provided by Steven Boyd (unpublished data). Invertebrate biomass from the SW averaged 2.7 times that of the NSS, and SW fish biomass averaged 3.0 times that of the NSS. If these biomass measures are related to chaetognath predator abundance, then it may be hypothesized that the NSS species are excluded from the SW by predators.

D. Salinity

On the basis of distributions restricted to oceanic regions, it has long been assumed that oceanic zooplankters are intolerant of even small changes in salinity. There is little experimental evidence to indicate the tolerance of oceanic zooplankters to salinity changes, but as discussed Chapter 4, crude experiments by Hopper (1960) suggest that oceanic zooplankters are tolerant of larger salinity changes than they would be exposed to in the open ocean. Gilfillan (1972), using respiration rates as a measure of stress, showed that the oceanic euphausiid, Euphausia pacifica, was tolerant of salinity values as low as 27 ppt, and his review 
of several previous studies also indicated a tolerance by oceanic zooplankters to salinities in the 20-30 ppt range. The observed salinity variations at the stations in the present study ranged from 32.8 to 36.8 ppt, indicating that salinity probably does not play a major role in limiting the horizontal distribution of zooplankters in this region, but further experimental work should be done to confirm this.

E. Oxygen concentration

Also as discussed in Chapter 4, there is no evidence to suggest that oxygen concetrations as low as the $3.0 \mathrm{ml} / 1$ values observed in this study have any limiting affect on zooplankton distributions.

F. Pressure

Pressure may also be an important factor involved in the regulation of the horizontal distribution of an organism if it orients its vertical distribution to temperature. As shown in Figure 4.1 , below $200 \mathrm{~m}$ in the SW, corresponding temperatures are found approximately 500-600 m deeper in the NSS. An organism tollowing an isotherm would experience a pressure change of about 50 atmospheres (equivalent to about 50 bars) in going from the SW to the NSS. There are no studies of the effects of pressure on chaetognaths, but studies on other marine organisms suggest that pressure changes as small as 50 atm have little or no short term effects. Little is known of the long term effects of increased or decreased pressure on birth and death rates, however.

Several studies indicated that an increase in pressure results in an increase in respiration for some midwater crustaceans (Napora, 1964; Pearcy and Sma11, 1968; Teal, 1971). These species were vertical migrators, and it was found that the increase in respiration due to 
increasing pressure is approximately cancelled by the decrease due to decreasing temperature with depth, resulting in a relatively constant respiration rate over the course of the migration (Teal, 1971).

If the SW chaetognaths are orienting their depths to temperature, then the increased pressure at the greater depth occupied in the NSS would result in an increase in respiration. This would result in a slower growth rate because more energy would be channeled into metabolism, leaving less for growth. It is not likely that this loss to respiration could be countered by increased feeding because food is less abundant in the NSS. The NSS species should not be adversely affected if this pressure effect is real.

G. Combinations of factors

It is likely that several of the above factors act in combination to limit the horizontal distributions of chaetognaths. Adequate assessment of the interactions of these factors will probably require the development of mathematical models of their effects on an organism's growth rate, egg production and mortality.

It is of interest here to discuss the relationships of horizontal distributions with water masses at this point. Since water masses in the oceans are identified by their temperature-salinity (T-S) properties, it has been tempting to argue that oceanic plankters are limited to particular water masses by their temperature and salinity characteristics. That this is the case is doubtful, and it has become clear that other features associated with water masses are responsible for the observed relationships (Bary, 1964; Backus et a1., 1969). While it is clear that plankton distributions are broadly related to water mass distributions, 
most of these distributions do not coincide exactly with those of water masses (MicGowan, 1971, Bieri, 1969). This also argues that we must look for other features of the pelagic environment besides $\mathrm{T}-\mathrm{S}$ characteristics to relate to the geographic abundance patterns.

G. Seasonal abundance patterns

If there are seasonal abundance differences for chaetognaths in the SW, they were not detectable from these samples. The spring abundance peaks for several of the chaetognaths in the NSS should be viewed as well documented given the results of this and Deevey's (1971) study. The spring abundance peaks correspond with the seasonal phytoplankton and zooplankton biomass peaks, indicating that increased food abundance is the principle cause. Surface water temperature is also at its minimum during this period. Lower temperatures generally result in slower growth rates and larger size at maturity, but, if these species show an increase in egg production with increasing body size as McLaren (1966) found for S. elegans, their birth rates could actually increase during this period, also resulting in increased abundances. There was no difference in predator abundance as measured by $M O C 10$ biomass between April and November of 1977 , thus arguing against a decrease in predation mortality in April as the cause of the abundance peaks. It is interesting to note that the epipelagic species showed the largest abundance peaks in April (Figure 5.4), which was to be expected since the greatest changes in temperature and food abundance occur in the upper $200 \mathrm{~m}$. S. maxima was the only bathypelagic species to show an abundance peak in April, while two of

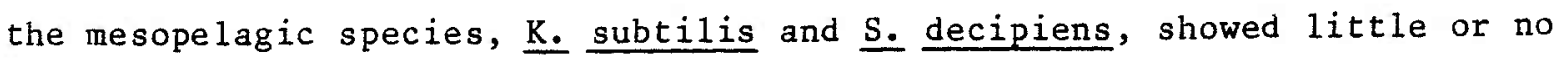
increase in April. Increased food abundance and/or decreased temperature 
are the most likely factors responsible for the spring chaetognath abundance peaks.

\section{Conclusions}

It is clear that gaining an understanding of the processes regulating the geographical and seasonal abundance patterns of zooplankton requires both laboratory experimentation and field studies. Until we are able to culture these oceanic plankters, however, we will have to rely upon field observations of their abundance patterns both for the development and testing of hypotheses regarding the regulation of the abundance patterns. Field studies in the future should be made as quantitative as possible in order to extract the maximum amount of information. I hope that I have convinced the reader of the utility of field sampling for the development of hypotheses, and it should be quite a test of our ingenuity to develop sampling programs to test these hypotheses. The use of quasi-experimental sites, such as Gulf Stream rings, should greatly increase our understanding of the processes regulating abundance patterns of pelagic organisms. 
CHAPTER 6

Summary and Recommendations 
I have documented the geographic, vertical and seasonal abundance patterns of chaetognaths in the western North Atlantic Ocean and shown how the sampling method can be used to develop hypotheses regarding the regulation of their spatial and temporal abundance patterns. Much further work is needed to test these hypotheses and to develop alternate hypotheses. I will briefly review the major findings of my work and offer some suggestions for future research.

Although the analysis in Chapter 3 suggested that sampling errors were not a major problem (or were at least quantifiable) for chaetognaths, further work is needed to confirm these preliminary analyses.

The analysis of avoidance suggested that only larger individuals of the larger species are undersampled because of avoidance. Laboratory experiments or in situ field observations are needed to test the assumptions made about the escape velocity and escape angle as well as to determine the stimuli which produce the escape reaction. Field experiments such as that described by Laval (1974) (see p. 72) can also be used to further quantify the avoidance capabilities of chaetognaths. If avoidance proves to be an important source of sampling error, then steps should be taken to reduce the stimuli produced by plankton nets.

The hypothesis that chaetognath width is the important dimension in mesh selection should be tested by a field program involving the use of nets with different mesh sizes. Such field studies are also needed to assess the fraction of each species' population which is not sampled by nets of a specific mesh size, such as the $0.333 \mathrm{~mm}$ mesh used in this study. Further studies of the spatial and temporal abundance patterns of small species such as $\underline{K}$. pacifica and $\underline{S}$. minima should employ a much finer 
mesh size than used in the present study.

Patchiness on a wide variety of space scales in the oceans can obscure the geographic and seasonal abundance patterns which we are trying to study. The effects of small scale patchiness can be reduced by increasing the volume of water filtered by plankton nets, and this is best accomplished by increasing the length of the tow rather than the mouth area of the net (Wiebe, 1971, 1972). Variations in abundance on larger space scales, such as those produced by Gulf Stream rings, were found to be large and require further study.

Subsampling with the Folsom plankton sample splitter produced greater variance than that expected from the binomial distribution and was a problem for some of the rarer species. Subsampling should be kept to a minimum, and if possible, aliquots should be taken such that between 50 and 100 individuals are counted for each species.

The observed vertical distributions were similar to those reported for these species from other regions. Diel vertical migrations were not detected for any of these species, but finer vertical resolution is needed within the upper $200 \mathrm{~m}$ to study diel vertical migrations of less than 100 m. Previous studies have shown that different developmental stages of some chaetognaths may have different migratory behaviors, so future work should involve differetiation of life stages.

Ontogenetic migrations were observed for several meso- and bathypelagic chaetognaths. These patterns should be confirmed from other samples, especially in other regions. Again, greater vertical resolution is needed in the upper $200 \mathrm{~m}$ to study ontogenetic migrations of epipelagic species. Analysis of the spatial and temporal variations in vertical 
distribution of the entire population of $\underline{S}$. lyra suggested that the se variations were related primarily to changes in the size frequency distribution of the population coupled with the ontogenetic migrations rather than to behavioral responses to differing environmental conditions. This hypothesis may explain variations in the vertical distributions of a number of other chaetognaths as well. Comparisons of the vertical distributions of particular size or sexual maturity classes may provide a means of understanding how individuals select their vertical distributions. Such an analysis is currently under way for S. 1yra. Studies of the vertical distributions of specific life stages of these chaetognaths in other oceanic regions should also contribute greatly to our understanding of how their vertical distributions are regulated.

Nine species were significantly more abundant in the Northern Sargasso Sea, seven species were significantly more abundant in the Slope Water, two species showed no significant differences and three species were too rare for analysis. Further sampling is needed from below $1000 \mathrm{~m}$ to adequately analyze the abundance differences for the bathypelagic species. The presence of many of the NSS species in the SW can be attributed to mixing processes as can the presence of a couple of SW species in the NSS. The identification of such expatriated populations requires the use of abundance estimates rather than presence/absence data and a quantitative knowledge of the mixing processes between hydrographic regions. Much more information is needed regarding the mixing processes in the western North Atlantic Ocean. In particular, the transfer of organisms from the SW to the NSS by the Western Boundary Undercurrent should be studied. 
Methods utilizing abundance estimates (e.g. factor analysis and correspondence analysis) should be employed for identification of pelagic communities rather than methods employing only presence/absence data (recurrent group analysis).

Seasonal abundance patterns were detected for many of the species in the NSS. The absence of seasonal abundance differences for other species in the SW and NSS may have been due to inadequate sampling. Further studies of the seasonal abundance patterns should employ biweekly to monthly sampling intervals and large spatial coverage at each time point. Such a study may not be practical, however, because of the large costs for ship time and labor which would be required.

Further studies in Gulf Stream rings may also provide much useful information for understanding the regulation of the spatial and temporal abundance patterns of chaetognaths. A good time series study from one ring is sorely needed.

It is clear that the testing of hypotheses regarding the regulation of the abundance patterns will require controlled laboratory experiments. Temperature and food availability are two factors which are known to affect the population dynamics of zooplankters and both differ significantly between the SW and NSS. Experimental studies are needed to determine the effects of temperature on the growth, reproduction and mortality of chaetognaths. Field studies of gut contents provide useful information concerning the selection of food by chaetognaths as can laboratory experiments. Further field studies combined with laboratory experimnts should allow us to understand how chaetognath abundance patterns are regulated. 
REFERENCES

0 
Ah1strom, E.H. 1954. Distribution and abundance of egg and larval populations of the Pacific Sardine. Fish. Bul1. 93(56): 83-140.

Alvarino, A. 1964. Bathymetric distribution of chaetognaths. Pacif. Sci. 18: $64-82$.

Alvarino, A. 1965. Chaetognaths. Oceanogr. Mar. Biol. Ann. Rev. 3: 115-194.

Alvarino, A. 1967. Bathymetric distribution of Chaetognatha, Siphonophorae, Medusae and Ctenophorae off San Diego, California. Pacif. Sci. 21: $274-285$.

Alvarino, A. 1969. Los quetognatos del Atlantico: Distribucion y notas esenciales de sistematica. Trab. Inst. Esp. Oceanogr. 37: 1-290.

Ange1, M.V. and M.J.R. Fasham. 1973. Sond Cruise 1965: Factor and cluster analyses of the plankton results, a general summary. J. Mar. Biol. Ass. U.K. 53: 185-231.

Ange1, M.V. and M.J.R. Fasham. 1974. Sond Cruise 1965: Further factor analyses of the plankton data. J. Mar. Biol. Ass. U.K. 54: 879-894.

Anraku, M. 1975. Microdistribution of marine copepods in a small inlet. Mar. Biol. 30: 79-87.

Aurich, V.H.J. 1971. Die verbreitung der Chaetognathen in gebiet des Nordatlantischen Strom-Systems. Ber. Dt. Wiss. Kommn. Meeresforsch 22: $1-30$.

Backus, R.H., J.E. Craddock, R.L. Haedrich and B.H. Robison. 1977. At lantic mesopelagic zoogeography. In: Fishes of the Western North Atlantic, Sears Foundation for Marine Research Memoir 1, Part 7: 266-287.

Backus, R.H., J.E. Craddock, R.L. Haedrich and D.L. Shores. 1969. Mesopelagic fishes and thermal fronts in the western Sargasso Sea. Mar. Biol. 3: 87-106.

Bagenal,M. 1955. A note on the relations of certain parameters following a logarithmic transformation. J. Mar. Biol. Ass. U.K. 34: 289-296.

Banse, K. 1964. On the vertical distribution of zooplankton in the sea. Progr. Oceanogr. 2: 53-125.

Barkley, R.A. 1964. The theoretical effectiveness of towed-net samplers as related to sampler size and to swimming speed of organisms. J. Cons. Perm. Int. Exp1or. Mer 29: 146-157.

Barkley, R.A. 1972. Selectivity of towed-net samplers. Fish. Bull. $70 \div 799-820$. 
Barnes, H. and S.M. Marshal1. 1951. On the variability of replicate plankton samples and some applications of 'contagious' series to the statistical distribution of catches over restricted periods. J. Mar. Biol. Assoc. U.K. 30: 233-263.

Bary, B.McK. 1964. Temperature, salinity and plankton in the eastern North Atlantic and coastal waters of Britain, 1957. IV. The species' relationship to the water body; Its role in distribution and in selecting and using indicator species. J. Fish. Res. Bd. Can. 21: 183-203.

Be, A.W.H., J.M. Forns and O.A. Roels. 1971. Plankton abundance in the North Atlantic Ucean. In: Fertility of the Sea, J.D. Costlow (ed.). Gordon and Breach Science Publishers, New York. Vol. 1: 17-50.

Belemishev, K.V. 1976. Ecology and Biogeography of the Open Ocean. Naval Oceanographic Office Translation, NOO T-25. 358 pp.

Bieri, R. 1959. The distribution of the planktonic Chaetognatha in the Pacific and their relationship to the water masses. Limnol. Oceanogr. 4: $1-28$.

Bigelow, H.B. 1926. Plankton of the offshore waters of the Gulf of Maine. Fish. Bul1. 40: 1-509.

Bigelow, H.B. and M. Sears. 1939. Studies of the waters of the continental shelf, Cape Cod to Chesapeake. III. A volumetric study of the zooplankton. Mem. Mus. Comp. Zool. 54: 181-378.

Boyd, S.H., P.H. Weibe and J.L. Cox. 1978. Limits of Nematoscelis megalops in the northwestern Atlantic in relation to Gulf Stream cold core rings. II. Physiological and biochemical effects of expatriation. J. Mar. Res. 36: 143-159.

Brinton, E. 1967. Vertical migration and avoidance capability of euphausiids in the California Current. Limnol. Oceanogr. 12: 451-483.

Lassie, R.M. 1962. Frequency distribution models in the ecology of plankton and other organisms. J. Anim. Ecol. 31: 65-92.

Cassie, R.M. 1963. Microdistribution of plankton. Oceanogr. Mar. Biol. Ann. Rev. 1: 223-252.

Cassie, R.M. 1968. Sample design. In: Zooplankton sampling. D.J. Tranter (ed.). UNESCO Monographs on Oceanographic Methodology 2: 105-121.

Chardy, P., M. Glemarec and A. Laurec. 1976. Application of intertia methods to benthic marine ecology: Practical implications of the basic options. Est. Coast. Mar. Sci. 4: 175-206.

Clarke, G.L. 1940. Comparative richness of zooplankton in coastal and offshore areas of the Atlantic. Biol. Bull., Woods Hole 78: 226-255. 
Clarke, G.L., E.L. Pierce and D.F. Bumpus. 1943. The distribution and reproduction of Sagitta elegans on Georges Bank in relation to the hydrographical conditions. Biol. Bul1. 85: 201-226.

Clutter, R.I. and M. Anraku. 1968. Avoidance of samplers. In: Zooplankton sampling. D.J. Tranter (ed.). UNESCO Monographs on Oceanographic Methodology 2: $57-76$.

Cosper, T.C. and M.R. Reeve. 1975. Digestive efficiency of the chaetognath Sagitta hispida Conant. J. Exp. Mar. Biol. Ecol. 17: 33-38.

Cox, J.L., P.H. Weibe, P. Ortner and S. Boyd. 1982. Seasonal development of subsurface chlorophy 11 maxima in Slope Water and Northern Sargasso Sea of the northwestern Atlantic Ocean. J. Biol. Oceanogr. In press.

Cushing, D.H. 1951. The vertical migration of planktonic Crustacea. Biol. Rev. 26: 158-192.

Dallot, S. and F. Ducret. 1969. Un Chaetognathe mesoplanktonique nouveau: Sagitta meglophthalena sp. n. Beaufortia 17: 13-20.

David, P.M. 1958. The distribution of the Chaetognatha of the Southern Ocean. Discovery Rept. 29: 199-228.

Deevey, G.B. 1971. The annual cycle in quantity and composition of the zooplankton of the Sargasso Sea off Bermuda. I. The upper $500 \mathrm{~m}$. Limnol. Oceanogr. 16: 219-240.

Deevey, G.B. and A.L. Brooks. 1971. The seasonal cycle in quantity and composition of the zooplankton of the Sargasso Sea off Bermuda. II. The surface to $2000 \mathrm{~m}$. Limnol. Oceanogr. 16: 927-y43.

Donaldson, H.A. 1975. Vertical distribution and feeding of sergestid shrimps (Decapoda: Natantia) collected near Bermuda. Mar. Biol. 31 : $37-50$.

Eakin, R.M. and J.A. Westiall. 1964. Fine structure of the eye of a chaetognath. J. Ce11 Biol. 21: 115-132.

Fager, E.W. 1957. Determination and analysis of recurrent groups. Ecology 38: $586-595$.

Fager, E.W. and J.A. McGowan. 1963. Zooplankton species groups in the North Pacific. Science 140: 453-460.

Fagetti, E. 1972. Bathymetric distribution of chaetognaths in the South Eastern Pacific Ocean. Mar. Biol. 17: 7-29.

Fasham, M.J.R., M.V. Angel and H.S.J. Roe. 1974. An investigation of the spatial pattern of zooplankton using the Longhurst-Hardy Plankton Recorder. J. Exp. Mar. Biol. Ecol. 16: 93-112. 
Feigenbaum, D.L. 1977. Nutritional ecology of the Chaetognatha with particular reference to external hair patterns, prey detection, and feeding. Ph.D. Dissertation, U. of Miami. $106 \mathrm{pp}$.

Feigenbaum, D.L. 1978. Hair-fan patterns in the Chaetognatha. Can. J. Zool.56(4): 536-546.

Figueiria, A.J.G. 1972. Occurrence of Eukrohnia bathypelagica Alvarino, 1962 (Chaetognatha) in the Atlantic waters of Canada. J. Fish. kes. Bd. Canada 29: 213-214.

Fisher, A. 1972. Entrainment of shelf water by the Gulf Stream northeast of Cape Hatteras. J. Geophys. Res. 77: 3248-3255.

Fleminger, A. and R.I. Clutter. 1965. Avoidance of towed nets by zooplankton. Limno1. Oceanogr. 10: 96-104.

Fleninger, A., J.D. Isaacs and J.G. Wyllie. 1974. Zooplankton biomass measurements from CalCOFI cruises of July 1955 to 1959 and remarks on comparison with results from October, January and April cruises of 1955 to 1959. California Cooperative Uceanic Fisheries Investigations, Atlas No. 21,118 pp.

Ford, W.L., J.R. Longard and R.E. Banks. 1952. On the nature, occurrence and origin of cold low salinity water along the edge of the Gulf Stream. J. Mar. Res. 11: 281-293.

Frost, B.W. 1974. Feeding processes at lower trophic levels in pelagic communities. In: The Biology of the Oceanic Pacific, C.B. Miller (ed.). Oregon State University Press, Corvallis. pp. 59-77.

Frost, B.W. and L.E. McCrone. 1974. Vertical distribution of zooplankton and myctophid fish at Canadian weather station $P$, with description of a new multiple net trawl. Proceedings of the International Conference on Engineering in the Ocean Environment. Halifax, Nova Scotia, Vol. 1: $159-165$.

Fuglister, F.C. 1972. Cyclonic rings formed by the Gulf stream 1965-66. In: Studies in Physical Oceanography- A Tribute to George Wust on his 80 th Birthday. A.L. Gordon (ed.), Gordon and Breach, New York. Vol. $1: 137-168$.

Gauch, H.G., R.H. Whittaker and T.R. Wentworth. 1977. A comparative study of reciprocal averaging and other ordination techniques. J. Ecol. 65: $157-174$.

Gehringer, J.W. 1962. The Gulf III and other modern high-speed plankton samplers. Rapp. P.-V. Reun. Cons. Perm. Int. Explor. Mer 153: 19-22.

Ghirarde11i, E. 1968. Some aspects of the biology of the chaetognaths. Adv. Mar. Biol. 67: 271-375. 
Gilfillan, E. 1972. Seasonal and latitudinal effects on the responses of Euphausia pacifica Hansen (Crustacea) to experimental changes of temperature and salinity. In: Biological Oceanography of the Northern North Pacific, A.Y. Takenouti (ed.), Idemitsu Shoten, Tokyo. pp. 443-463.

Gophen, M. and R.P. Harris. 1981. Visual predation by a marine cyclopoid copepod, Corycaeus anglicus. J. Mar. Biol. Assoc. U.K. 61: 391-399.

Grant, G.C. 1963. Investigations of inner continental shelf waters off lower Chesapeake Bay. Part IV. Descriptions of the Chaetognatha and a key to their identification. Chesapeake Sci. 4: 107-119.

Grant, G.C. 1967. The geographic distribution and taxonomic variation of Sagitta serratodentata Krohn 1853 and Sagitta tasmanica Thomson 1947 in the North Atlantic Ocean. Ph.D. dissertation, U. Rhode Island, 116 pp.

Grant, G.C. 1977. Seasonal distribution and abundance of the Chaetognatha in the lower Chesapeake Bay. Est. Coast. Mar. Sci. 5: 809-824.

Grice, G.D. and A.D. Hart. 1962. The abundance, seasonal occurrence and distribution of the epizooplankton between lNew York and Bermuda. Ecol. Monogr. 32: 287-309.

Harder, W. 1968. Reactions of plankton organisms to water stratification. Limnol. Uceanogr. 13: 156-168.

Harman, H.H. 1967. Modern Factor Analysis. The University of Chicago Press, Chicago.

Haury, L.R. 1976. Small scale pattern of a California Current zooplankton assemblage. Mar. Biol. 37: 137-157.

Haury, L.R., J.A. McGowan and P.H. Weibe. 1978. Patterns and processes in the time-space scales of plankton distributions. In: Spatial Pattern in Plankton Communities, J.H. Steele (ed.), Plenum Press, New York: $277-327$.

Heron, A.C. 1968. Plankton gauze. In: Zooplankton sampling. D.J. Tranter (ed.). UNESCO Monographs on Oceanographic Methodology 2: 19-25.

Hill, M.0. 1973. Reciprocal averaging: An eigenvector method of ordination. J. Ecol. 61: 237-249.

Hill, M.0. 1974. Correspondence analysis: A neglected multivariate method. App1. Statist. 23: 340-354.

Hopper, S.F. 1960. The resistance of marine zooplankton of the Caribbean and South Atlantic to changes in salinity. Limnol. Oceanogr. 5: 43-47.

Horridge, G.A. and P.S. Boulton. 1967. Prey detection by Chaetognatha via a vibration sense. Proc. Roy. Soc. B 168: 413-419. 
Hulburt, E.M. 1966. The distribution of phytoplankton, and its relationship to hydrography, between southern New $k$ England and Venezuela. J. Mar. Res. 24: 67-81.

Huntsman, A.G. 1919. A special study of the Canadian chaetognaths, their distribution, etc., in the waters of the eastern coast. Rep. Dept. Nav. Serv. Can., Can. Fish. Exped. 1914-15: 421-485.

Iselin, C. O'D. 1936. A study of the circulation of the western North At lantic. Pap. Phys. Oceanogr. Meteor. 4(4): 1-101.

Iselin, C. O'D. 1939. The influence of vertical and lateral turbulence on the characteristics of waters at mid-depth. Trans. Amer. Geophys. Un. 1939: $414-417$.

Jahn, A.E. 1976. On the midwater fish faunas of Gulf Stream rings, with respect to habitat differences between Slope Water and Northern Sargasso Sea. Ph.D. thesis. Woods Hole Oceanographic Institution. 173 pp.

Jahn, A.E. and R.H. Backus. 1976. On the mesopelagic fish faunas of Slope Water, Gulf Stream, and northern Sargasso Sea. Deep-Sea Res. 23: $223-234$.

Jakobsen, T. 1971. On the biology of Sagitta elegans Verrill and Sagitta setosa J. Muller in Inner Oslofjord. Norw. J. Zool. 19: 201-225.

James, R. 1979. Antarctic chaetognaths from a time station near Elephant Island. Meeresforsch. 27: 282-287.

King, K.R. 1979. The life history and vertical distribution of the chaetognath, Sagitta elegans, in Dabob Bay, Washington. J. Plankton Res. 1: 153-167.

Kinne, 0. 1970. Temperature: Animals: Invertebrates. In: Marine Ecology, 0. Kinne (ed.). Wiley-Interscience. Vo1. I, Part I: 407-514.

Kitou, M. 1966. Chaetognaths collected on the Fifth Cruise of the Japanese Expedition of the deep seas. La Mer 4: 169-177.

Kitou, M. 1967. Distribution of Eukrohnia hamata (Chaetognatha) in the western North Pacific. Inf. Bull. Planktol. Japan. Comm. Number of Dr. Y. Matsue: 91-96.

Kolosova, Ye. G. 1972. Vertical distribution and daily migrations of Chaetognatha in the tropical Pacific. Okeanologiya 12: 129-136.

Kotori, M. 1972. Vertical distribution of chaetognaths in the northern North Pacific Ocean and Bering Sea. In: Biological Oceanography of the Northern North Pacific Ocean, A.Y. Takenouti (ed.), Idemitsu Shoten (Tokyo): 291-308. 
Krebs, C.J. 1978. Ecology: The Experimental Analysis of Distribtion and Abundance. Harper and Row, New York. 678 pp.

Kuhlmann, D. 1977. Laboratory studies on the feeding behavior of the chaetognaths Sagitta setosa J. Muller and S. elegans Verrill with special reference to fish eggs and larvae as food organisms. Meeresforsch. 25: 163-171.

Lai, D.Y. and P.L. Richardson. 1977. Distribution and movement of Gulf Stream rings. J. Phys. Oceanogr. 7: 670-683.

Lasker, R. 1975. Field criteria for survival of anchovy larvae: The relationbetween inshore chlorophyll maximum layers and successful firstfeeding. Fish. Bul1. 73: 453-462.

Laval, P. 1974. Un modele mathematique de 1 'evitement d'un filet a plancton, son application pratique, et sa verification indirecte en recourant au parasitione de l'amphipods hyperids Vibilia armata Bovallius. J. Exp. Mar. Biol. Ecol. 14: 57-87. English translation from Dept. of the Environment, Marine Ecology Laboratory, Dartmouth, Nova Scotia. Fisheries and Marine Service Translation Series No. 3116.

Lebour, M.V. 1922. The food of plankton organisms. J. Mar. Biol. Assoc. U.K. $12: 644-677$.

Lebour, M.V. 1923. The food of plankton organisms. II. J. Mar. Biol. Assoc. U.K. 13: 70-92.

Lee, A.H. 1970. The T-S structure, circulation and mixing in the Slope Water region east of the Scotian Shelf. Ph.D. thesis, Dalhousie University. $191 \mathrm{pp}$.

Lewis, J.B. and A.G. Fish. 1969. Seasonal variation of the zooplankton fauna of surface waters entering the Caribbean Sea at Barbados. Carib. J. Sci. 9: 1-24.

Longhurst, A.R. and D.L.R. Siebert. 1967. Skill in the use of Folsom's plankton sample splitter. Limnol. Oceanogr. 12: 334-335.

Lumb, F.E. 1964. The influence of cloud on hourly amount of total solar radiation at the sea surface. Quart. J. Roy. Meteor. Soc. 90: 43-56.

Lyons, H.W. 1976. Seasonality in Central North Pacific chaetognaths. Ph.D. dissertation. U. California, San Diego. $143 \mathrm{pp}$.

Mackintosh, N.A. 1937. The seasonal circulation of the Antarctic macroplankton. Disc. Rept. 16: 365-412.

Mauchline, J. and L.R. Fisher. 1969. The biology of euphausiids. Adv. Mar. Biol. 7: 1-454. 
McElroy, P.T. and W. Smith. 1975. Clustering of volume reverberation spectra: An application of correspondence analysis. J. Acoust. Soc. Amer. 58: 1234-1256.

McEwen, G.F., M.W. Johnson and Th.R. Folsom. 1954. A statistical study of the performance of the Folsom plankton sample splitter, based upon test observations. Arch. Meteorol. Geophys. Bioclimatol., Ser. A, 6 : 502-527.

McGowan, J.A. 1960. The relationship of the distribution of the planktonic worm, Poeobius meseres Heath, to the water masses of the North Pacific. Deep-Sea Res. 6: 125-139.

McGowan, J.A. 1971. Oceanic biogeography of the Pacific. In: Micropaleontology of the Oceans, B.M. Funnell and W.R. Reidel, (eds.). Cambridge University Press, London: 3-74.

McGowan, J.A. 1974. The nature of oceanic ecosystems. In: Biology of the Oceanic Pacific, C.B. Miller (ed.). Oregon State University Press, Corvallis: $9-28$.

Mcharen, I.A. 1963. Effects of temperature on growth of zooplankton, and the adaptive value of vertical migration. J. Fish. Res. Bd. Can 20: $685-727$.

McLaren, I.A. 1966. Adaptive significance of large size and long life of the chaetognath Sagitta elegans in the Arctic. Ecology 47: 852-855.

McLaren, I.A. 1969. Population and production ecology of zooplankton in Ogae Lake, a landlocked fjord on Baffin Island. J. Fish. Res. Bd. Can. 26: $1485-1559$.

McLaren, I.A. 1974. Demographic strategy of vertical migration by a marine copepod. Am. Natur. 108: 91-102.

McLellan, H.J. 1956. On the distinctness and origin of the slope Water off the Scotian Shelf and its easterly flow south of the Grand Banks. J. Fish. Res. Bd. Canada 14: 213-239.

McLe1land, J.A. 1980. Notes on the northern Gulf of Mexico occurrence of $\frac{\text { Sagitta friderici Ritter-Zahony (Chaetognatha). Gulf Res. Rep. 6: }}{343-348}$ :

Menze1, D.W. and J.H. Ryther. 1960. The annual cycle of primary production in the Sargasso Sea off Bermuda. Deep-Sea Res. 6: 351-367.

Merrett, N.R. and H.S.J. Roe. 1974. Patterns and selectivity in the feeding of certain mesopelagic fishes. Mar. Biol. 28: 115-126.

Michel, H.B. and M. Foyo. 1976. Siphonophora, Heteropoda, Copepoda, Euphausiacea, Chaetognatha and Salpidae. In: Carbbean Zooplankton, H.B. Michel, M. Foyo and D.A. Haagensen. U.S. Government Printing Office, pp. 1-549. 
Miller, C.B. 1969. Some environmental consequences of vertical migration. $\mathrm{Ph} . \mathrm{D}$. thesis. University of California, San Diego. $308 \mathrm{pp}$.

Mironov, G.N. 1960. The food of plankton predators. II. Food of Sagitta. Trud. Sevastopol Biol. Stansii 13: 78-88.

Moller, H. 1980. A summer survey of large zooplankton, particularly scyphomedusae, in the North Sea and Baltic. Meeresforsch. 28: 61-68.

Moore, H.B. 1949. The zooplankton of the upper waters of the Bermuda area of the North Atlantic. Bu11. Bingham Oceanogr. Col1. 12(2): 1-97.

Moore, H.B. 1955. Variations in temperature and light response within a plankton population. Biol. Bul1., Woods Hole 108: 175-181.

Moore, H.B., H. Owre, E.C. Jones and T. Dow. 1953. Plankton of the Florida Current. III. The control of the vertical distribution of zooplankton in the daytime by light and temperature. Bull. Mar. Sci. Gulf and Carib. 3: 83-95.

Moore, E. and F. Sander, 1977. A study of the offshore zooplankton of the tropical western Atlantic near Barbados. Ophelia 16: 77-96.

Moore, E. and F. Sander. 1979. A comparative study of zooplankton from oceanic, shelf, and harbor waters of Jamaica. Biotropica 11: 196-206.

Morgan, C.W. and J.M. Bishop. 1977. An example of Gulf Stream eddy-induced water exchange in the Mid-Atlantic Bight. J. Phys. Oceanogr. 7: 472-479.

Motoda, S. and M. Anraku. 1954. Daily change of vertical distribution of plankton animals near western entrance to the Tsugaru Strait, northern Japan. Bul1. Fac. Fish., Hokkaido Univ. 5: 15-19.

Motoda, S. and M. Anraku. 1955. Further observation on the daily change in amount of catches of plankton animals in vertical hauls. Bull. Fac. Fish.,Hokkaido Univ. 6: 15-18.

Mullin, M.M. and E.R. Brooks. Some consequences of distributional heterogeneity of phytoplankton and zooplankton. Limnol. Oceanogr. 21: 784-796.

Murphy, G.I. and R.I. Clutter. 1972. Sampling anchovy larvae with a plankton purse seine. Fish. Bull. 70:789-798.

Nagasawa, S. and R. Marumo. 1972. Feeding of a pelagic chaetognath, Sagitta nagae Alvarino in Suruga bay, central Japan. J. Oceanogr. Soc. Japan 28: 181-186.

Nagasawa, S. and R. Marumo. 1976. Further studies of the feeding habits of Sagitta nagae Alvarino in Suruga Bay, central Japan. J. Oceanogr. Soc. Japan 32: 209-218. 
Nair, V.R. 1978. Bathymetric distribution of chaetognaths in the Indian Ocean. Indian J. Mar. Sci. 7: 276-282.

Napora, T.A. 1964. The effects of hydrostatic pressure on the prawn Systellapsis debilis. Narragansett Mar. Lab. Occ. Pub1. 2: 92-94.

Newbury, T.K. 1972. Vibration perception by chaetognaths. Nature 236: $459-460$.

Nie, N.H., C.H. Hal1, J.G. Jenkins, K. Steinbrenner, and D.H. Brent. 1975. Statistical Package for the Social Sciences. McGraw Hill Book Co., New York. 673 pp.

O'Day, W.T. and B.G. Nafpaktitis. 1967. A study of the effects of expatriation on the gonads of two myctophid fishes in the North At lantic Ocean. Bul1. Mus. Comp. Zool. Harvard 136: 77-90.

Omori, M. 1974. The biology of the pelagic shrimps of the ocean. Adv. Mar. Biol. 12: 233-324.

Omori, M., R. Marumo and Y. Aizawa. 1965. A 160-cm opening-closing plankton net. J. Oceanogr. Soc. Japan 21: 245-252.

Ortner, P.B., E.M. Hulburt and P.H. Wiebe. 1979. Phytohydrography, Gulf Stream rings, and herbivore habitat contrasts. J. Exp. Mar. Biol. Ecol. 39: 101-124.

Ortner, P.B., P.H. Wiebe and J.L. Cox. 1980. Relationships between oceanic epizooplankton distributions and the seasonal deep chlorophyll maximum in the Northwestern Atlantic Ocean. J. Mar. Res. 38: 507-531.

Ortner, P.B., P.H. Wiebe, L. Haury and S. Boyd. 1978. Variability in zooplankton biomass distribution in the Northern Sargasso Sea: The contribution of Gulf Stream cold core rings. Fish. Bu11. 76: 323-334.

Owre, H.B. 1960. Plankt on of the Florida Current. Part VI. The Chaetognatha. Bull. Mar. Sci. Gulf Carib. 10: 255-322.

Pearcy, W.G. and L.F. Small. 1968. Effects of pressure on the respirarion of vertically migrating crustaceans. J. Fish. Res. Bd. Can. 25: 1311-1316.

Pearre, S. 1973. Vertical migration and feeding in Sagitta elegans Verrill. Ecology 54: 300-314.

Pearre, S. 1974. Ecological studies of three West-Mediterranean chaetognaths. Inv. Pesq. 38: 325-369.

Pearre, S. 1976. A seasonal study of the diets of three sympatric chaetognaths. Inv. Pesq. 40: 1-16.

Pearre, S. 1979. Problems of detection and interpretation of vertical migration. J. Plank. Res. 1: 29-44. 
Pearre, S. 1980. Feeding by Chaetognatha: The relationship of prey size to predator size in several species. Mar. Ecol. Progr. Ser. 3: 125-134.

Pearre, S. 1981. Feeding by Chaetognatha: Energy balance and importance of various components of the diet of Sagitta elegans. Mar. Ecol. Progr. Ser. 5: 45-54.

Pierrot-Bults, A.C. 1975. Taxonomy and zoogeography of Sagitta planctonis Steinhaus, 1896 (Chaetognatha) in the Atlantic Ocean. Beaufortia 23: $27-51$.

Rakusa-Suszczewski, S. 1969. The food and feeding habits of Chaetognatha in the seas around the British Isles. Pol. Archiv. Hydrobiol. 16: 213-232.

Reed, R.K. 1977. On estimating insolation over the ocean. J. Phys. Oceanogr. 7: 482-485.

Reeve, M.R. 1964. Feeding of zooplankton, with special reference to some experiments with Sagitta. Nature 201: 211-213.

Reeve, M.R. 1966. Observations on the biology of a chaetognath. In: Some Contemporary Studies in Marine Science, H. Barnes (ed.), George Allen and Unwin Ltd., London. pp. 613-630.

Reeve, M.R. 1970a. Complete cycle of development of a pelagic chaetognath in culture. Nature 227: 381 .

Reeve, M.R. 1970b. The biology of Chaetognatha I. Quantitative aspects of growth and egg production in Sagitta hispida. In: Marine Food Chains, J.H. Steele (ed.) Oliver and Boyd, Edinburgh: 168-189.

Reeve, M.R. 1977. Prey detection in the Chaetognatha: Response to a vibrating probe and experimental determination of attack distance in large aquaria. Limnol. Oceanogr. 22: 1052-1058.

Reeve, M.R. and L.D. Baker. 1975. Production of two planktonic carnivores (chaetognath and ctenophore) in south Florida inshore waters. Fish. Bu11. 73: 238-248.

Reeve, M.R. and M.A. Walter. 1972. Conditions of culture, food-size selection, and the effects of temperature and salinity on growth rate and generation time in Sagitta hispida Conant. J. Exp. Mar. Biol. Ecol. 17: 39-46.

Reid, J.L., E. Brinton, A. Fleminger, E.L. Venrich and J.A. McGowan. 1978. Ocean circulation and marine life. In: Advances in Oceanograpny. $H$. Charnock and G. Deacon, (eds.). Plenum Publishing Co.: 65-130.

Ricker, W.E. 1973. Linear regressions in fishery research. J. Fish. Res. Board Can. 30: 409-434. 
Riley, G.A. 1956. Oceanography of Long Island Sound, 1952-1954. IX. Production and utilization of organic matter. Bull. Bingham Oceanogr. Col1. 15: 324-344.

Robinson, M.K., R.A. Bauer and E.H. Schroeder. 1979. Atlas of North Atlantic-Indian Ocean Monthly Mean Temperatures and Mean Salinities of the Surface Layer. Naval Oceanographic Office Reference Publication 18. Naval Oceanographic Office, Bay St. Louis, Mississippi.

Roe, H.S.J. 1974. Observations on the diurnal vertical migrations of an oceanic animal community. Mar. Biol. 28: 99-113.

Rohlf, F.J. and R.R. Soka1. 1969. Statistical Tables. W.H. Freeman and Co. San Francisco. 253 pp.

Rossby, C.-G. 1936. Dynamics of steady ocean currents in the light of experimental fluid mechanics. Pap. Phys. Oceanogr. Meteor. 5(1): 1-43.

Rummel, R.J. 1970. Applied Factor Analysis. Northwestern University Press, Evanston.

Russe11, F.S. 1931. The vertical distribution of marine macroplankton.X. Notes on the behavior of Sagitta in the Plymouth area. J. Mar. Biol. Ass. U.K. 17: 391-407.

Russe11, F.S. 1932. On the biology of Sagitta. The breeding and growth of Sagitta elegans Verrill in the Plymouth area 1930-31. J. Mar. Biol. Ass. U.K. 18: 147-160.

Ryther, J.H. 1963. Geographic variations in productivity. In: The Sea, M.N. Hill (ed.). Interscience Publishers, New York. Vol. 2: 347-380.

Sameoto, D.D. 1971. Life history, ecological production, and an empirical mathematical model of the population of Sagitta elegans in St. Margaret's Bay, Nova Scotia. J. Fish. Res. Bd. Canada 28: 971-985.

Sameoto, D.D. 1973. Annual life cycle and production of the chaetognath Sagitta elegans in Bedford Basin, Nova Scotia. J. Fish. Res. Bd. Canada 30: 333-344.

Sameoto, D.D. 1978. Zooplankton sample variation on the Scotian Shelf. J. Fish. Res. Board Can. 35: 1207-1222.

Sameoto, D.D., L.O. Jaroszynski, and W.B. Fraser. 1977. A multiple opening and closing plankton sampler based on the MOCNESS and N.I.O. nets. J. Fish. Res. Bd. Can. 34: 1250-1235.

Sands, N.J. 1978. Ecological studies on the deep-water pelagic community of Korsfjorden, western Norway: Comparison of the catch of the more numerous species by two different nets. Sarsia 63: 237-246.

Sands, N.J. 1980. Ecological studies on the deep-water community of 
Korsfjorden, western Norway: Population dynamics of the chaetognaths from 1971-1974. Sarsia 65: 1-12.

Saville, A. 1958. Mesh selection in plankton nets. J. Cons. Perm. Int. Explor. Mer 23: 192-201.

Schmitz, W.J., J.F. Price, P.L. Richardson, W.B. Owens, D.C. Webb, R.E. Cheney and H.T. Rossby. 1981. A preliminary exploration of the Gulf Stream system with SOFAR floats. J. Phys. Oceanogr. 11: 1194-1204.

Singarajah, K.V. 1966. Pressure sensitivity of the chaetognath Sagitta setosa. Comp. Biochem. Physiol. 19: 475-478.

Singarajah, K.V. 1975. Escape reactions of zooplankton: Effects of light and turbulence. J. Mar. Biol. Assoc. U.K. 55: 627-639.

Smith, P.E., R.C. Counts and R.I. Clutter. 1968. Changes in filtering efficiency of plankt on nets due to clogging under tow. J. Cons. Perm. Int. Explor. Mer 32: 232-248.

Sokal, R.R. and F.J. Roh1f. 1969. Biometry: The Principles and Practice of Statistics in Biological Research. W.H. Freeman and Co. San Francisco. $776 \mathrm{pp}$.

Soutar, A. and J.D. Isaacs. 1974. Abundance of pelagic fish during the 19 th and 20 th centuries as recorded in anaerobic sediments off the Californias. Fish. Bul1. 72: 257-273.

Southward, A.J. 1980. The western English Channe1- an inconstant ecosystem? Nature 285: 361-366.

Steele, J.H. 1974a. Spatial heterogeneity and population stability. Nature 248: 83 .

Steele, J.H. 1974b. The Structure of Marine Ecosystems. Harvard University Press, Cambridge, Mass. 128 pp.

Stepien, J.C. 1980. The occurrence of chaetognaths, pteropods and euphausiids in relation to deep flow reversals in the straits of Florida. Deep Sea Res. 27A: 987-1011.

Stommel, H. 1958. The abyssal circulation. Deep-Sea Res. 5: 80-82.

Stone, J.H. 1969. The Chaetognatha community of the Agulhas Current: Its structure and related properties. Ecol. Monogr. 39: 433-563.

Sullivan, B.K. 1980. In situ feeding behavior of Sagitta elegans and Eukrohnia hamata (Chaetognatha) in relation to the vertical distribution and abundance of prey at Ocean Station "P". Limnol. Oceanogr. 25 : 317-326.

Sverdrup, H.U., M.W. Johnson and R.H. Fleming. 1942. The Oceans. 
Prentice-Ha11, Inc., 1087 pp.

Szyper, J.P. 1978. Feeding rate of the chaetognath Sagitta enflata in nature. Est. Coast. Mar. Sci. 7: 567-575.

Teal, J.M. 1971. Pressure effects on the respiration of vertically migrating decapod crustacea. Am. Zool. 11: 571-576.

Teil, H. 1975. Correspondence factor analysis: An outline of its method. Math. Geol. 7: 3-30.

Teissier, G. 1948. La relation d'allometries; sa significance statistique et biologique. Biometrics 4: 14-48.

The Ring Group. 1981. Gulf Stream cold-core rings: Their physics, chemistry, and biology. Science 212: 1091-1100.

Thomson, J.M. 1947. The Chaetognatha of South-eastern Australia. Bu11. Coun. Scient. Ind. Res. 222: 4-43.

Timonin, A.G. 1969. The structure of pelagic associations: The quantitative relationships between different trophic groups of plankton in frontal zones of the tropical ocean. Okeanologiya 9: 846-856.

Tranter, D.J. 1967. A formula for the filtration coefficient on a plankton net. Aust. J. Mar. Freshwat. Res. 18: 113-121.

Tranter, D.J. and P.E. Smith. 1968. Filtration performance. In: Zooplankton sampling. D.J. Tranter (ed.). UNESCO Monographs on Oceanographic Methodology 2: 27-56.

Tranter, D.J., J.D. Kerr and A.C. Heron. 1968. Effects of hauling speed on zooplankt on catches. Aust. J. Mar. Freshwat. Res. 19: 65-75.

Uda, M. 1961. Fisheries oceanography in Japan, especially on the principles of fish distribution, concentration, dispersal and fluctuation. CalCOFI Progress Report 8: 25-31.

Vannucci, M. 1968. Loss of organisms through the meshes. In: Zooplankton sampling. D.J. Tranter (ed.). UNESCO Monographs on Oceanographic Methodology 2: 77-86.

Venrick, E.L. 1971a. The statistics of subsampling. Limnol. Oceanogr. 16: $811-818$.

Venrick, E.L. 1971b. Recurrent groups of diatom species in the North Pacific. Ecology 52: 614-625.

Vinogradov, M.E. 1970. Vertical distribution of the oceanic zooplankton. Israel Program for Scientific Translations Ltd., 339 pp. 
Waterman, T.H. and D.A. Berry. 1967. Evidence for diurnal vertical plankton migration below the photic zone. Am. Zool. 7: 417.

Welsh, J.H., F.A. Chace and R.F. Nunnemacher. 1937. The diurnal migration of deep-water animals. Biol. Bull., Woods Hole 73: 185-196.

Whittaker, R.H. and C.W. Fairbanks. 1958. A study of plankton copepod communities in the Columbia Basin, Southeast Washington. Ecology 39: 46-65.

Wiebe, P.H. 1970. Small scale spatial distribution in oceanic zooplankton. Limnol. Oceanogr. 15: 205-217.

Wiebe, P.H. 1971. A computer model study of zooplankton patchiness and its effects on sampling error. Limnol. Oceanogr. 16: 29-38.

Wiebe, P.H. 1972. A field investigation of the relationship between length of tow, size of net and sampling error. J. Cons. Int. Explor. Mer 34: $268-275$.

Wiebe, P.H. and S.H. Boyd. 1978. Limits of Nematoscelis megalops in the northwestern Atlantic in relation to Gulf Stream cold core rings. I. Horizontal and vertical distributions. J. Mar. Res. 36: 119-142.

Wiebe, P.H. and W.R. Holland. 1968. Plankton patchiness: Effects on repeated net tows. Limnol. Oceanogr. 13: 315-321.

Wiebe, P.H., G.D. Grice and E. Hoagland. 1973. Acid-iron waste as a factor affecting the distribution and abundance of zooplankton in the New York Bight II. Spatial variations in the field and implications for monitoring sudies. Est. Coast. Mar. Sci. 1: 51-64.

Wiebe, P.H., S.H. Boyd, B.M. Davis and J.L. Cox. In press. Avoidance of towed nets by the euphausiid Nematoscelis megalops. Fish. Bull.

Wiebe, P.H., K.H. Burt, S.H. Boyd and A.W. Morton. 1976. A multiple opening/closing net and environmental sensing system for sampling zooplankton. J. Mar. Res. 34: 313-326

Williams, W.T. 1971. Principles of clustering. Ann. Rev. Ecol. Syst. 2 : $303-326$.

Winsor, C.P. and G.L. Clarke. 1940. A statistical study of variation in the catch of plankton nets. J. Mar. Res. 3: 1-34.

Winsor, C.P. and L.A. Walford. 1936. Sampling variations in the use of plankton nets. J. Cons. Int. Explor. Mer. 11: 190-204.

Wishner, K.F. 1980. Aspects of the community ecology of deep-sea, benthopelagic plankton, with special attention to gymnopleid copepods. Nar. Biol. 60: 179-187. 
Worthington, L.V. 1959. The $18^{\circ}$ water in the Sargasso Sea. Deep-Sea Res. 5: 297-305.

Worthington, L.V. 1976. On the North Atlantic Circulation. The Johns Hopkins Oceaographic Studies No. 6. The Johns Hopkins University Press. 110 pp.

Wright, W.R. 1976a. The limits of shelf water south of Cape Cod, 1941 to 1972. J. Mar. Res. 34: 1-14.

Wright, W.R. 1976b. Physical oceanography. In: Summary of Environmental Information on the Continental Slope-Canadian United States Border to Cape Hatteras, North Carolina. Submitted to the Bureau of Land Management Marine Minerals Division by Research Institute of the Gulf of Maine (TRIGOM), South Portland, Maine. 110 pp.

Wright, W.R. and C.E. Parker. 1976. A volumetric temperature/salinity census for the Middle Atlantic Bight. Limnol. Oceanogr. 21: 563-571.

Zo, Z. 1973. Breeding and growth of the chaetognath Sagitta elegans in Bedford Basin. Limnol. Oceanogr. 18:750-756. 